FLAVOUR(267104)-ERC-45

\title{
Towards the Identification of New Physics through Quark Flavour Violating Processes*
}

\author{
Andrzej J. Buras and Jennifer Girrbach \\ TUM-IAS, Lichtenbergstr. 2a, D-85748 Garching, Germany \\ Physik Department, TUM, D-85748 Garching, Germany
}

\begin{abstract}
We outline a systematic strategy which should help in this decade to identify New Physics (NP) beyond the Standard Model (SM) by means of quark flavour violating processes and thereby to extend the picture of short distance physics down to the scales as short as $10^{-20} \mathrm{~m}$ and even shorter distance scales corresponding to energies of $100 \mathrm{TeV}$. Rather than using all possible flavour violating observables that will be measured in the coming years at the LHC, SuperKEKB and in Kaon physics dedicated experiments at CERN, J-PARC and Fermilab, we concentrate on those observables that are theoretically clean and very sensitive to NP. Assuming that the data on the selected observables by us will be very precise, we stress the importance of correlations between these observables as well as of future precise calculations of non-perturbative parameters by means of lattice QCD simulations with dynamical fermions. Our strategy consists of twelve steps which we will discuss in detail illustrating possible outcomes with the help of the SM, models with constrained Minimal Flavour Violation (CMFV), MFV at large and models with tree-level FCNCs mediated by neutral gauge bosons and scalars. We also briefly summarize the status of a number of concrete models. We propose DNA-charts that exhibit correlations between flavour observables in different NP scenarios. The models with new left-handed and/or right-handed currents and nonMFV interactions can be distinguished transparently in this manner. We emphasize the important role of the stringent CMFV relations between various observables as standard candles of flavour physics. The pattern of deviations from these relations may help in identifying the correct NP scenario. The success of this program will be very much facilitated through direct signals of NP at the LHC even if LHC will not be able to probe the physics at scales shorter than $4 \times 10^{-20} \mathrm{~m}$. We also emphasize the importance of lepton flavour violation, electric dipole moments and $(g-2)_{e, \mu}$ in these studies.
\end{abstract}

*Review article to be submitted for publication in Reports on Progress in Physics 


\section{Contents}

1 Overture

2 Strategy

2.1 Setting the Scene . . . . . . . . . . . . . . . 7

2.2 Towards New Standard Model in 12 Steps . . . . . . . . . . . . . . . 9 9

2.3 Correlations between Observables . . . . . . . . . . . . . . . 9 9

3 Theoretical Framework $\quad 12$

3.1 Preliminaries ....................... 12

3.2 Constrained Minimal Flavour Violation $(\mathrm{CMFV})$. . . . . . . . . . . 14

3.3 CMFV Relations as Standard Candles of Flavour Physics . . . . . . . . 15

3.4 Minimal Flavour Violation at Large $(\mathrm{MFV})$. . . . . . . . . . . . . . 16

3.5 Simplest Models with non-MFV Sources . . . . . . . . . . . . . . . 18

3.6 The $U(2)^{3}$ Models . . . . . . . . . . . . . . . . . . . . . . . . . . . . . . . . . . . . 18

3.7 Tree-Level Gauge Boson and Scalar Exchanges . . . . . . . . . . . . . . 18

4 Classifying Correlations between various Observables 19

5 Searching for New Physics in twelve Steps 22

5.1 Step 1: The CKM Matrix from tree level decays . . . . . . . . . . 22

5.2 Step 2: Improved Lattice Calculations of Hadronic Parameters . . . . . 26

5.3 Step 3: $\Delta F=2$ Observables . . . . . . . . . . . . . . 27

5.3.1 Contributing operators . . . . . . . . . . 27

5.3.2 Standard Model Results . . . . . . . . . . . . . 29

5.3.3 Going Beyond the Standard Model . . . . . . . . . . 33

5.3.4 Constrained Minimal Flavour Violation (CMFV) . . . . . . . 35

5.3.5 2HDM with MFV and Flavour Blind Phases $\left(2 \mathrm{HDM}_{\overline{\mathrm{MFV}}}\right)$. . . . 41

5.3.6 Tree-Level Gauge Boson Exchanges . . . . . . . . . . . . . . . 44

5.3.7 Tree-Level Scalar Exchanges . . . . . . . . . . . . . . . . . . 49

5.3.8 Implications of $U(2)^{3}$ Symmetry . . . . . . . . . . . . . . . . . . 50

5.4 Step 4: $\mathcal{B}\left(B_{s, d} \rightarrow \mu^{+} \mu^{-}\right)$and $\mathcal{B}\left(B_{s, d} \rightarrow \tau^{+} \tau^{-}\right) \ldots \ldots$ 
5.4 .1 Preliminaries $\ldots \ldots \ldots \ldots \ldots \ldots \ldots$

$5.4 .2 \quad$ Basic Formulae . . . . . . . . . . . . . . . . 5 53

5.4.3 Standard Model Results and the Data . . . . . . . . . 56

$5.4 .4 \quad \mathrm{CMFV} \ldots \ldots \ldots \ldots \ldots \ldots$

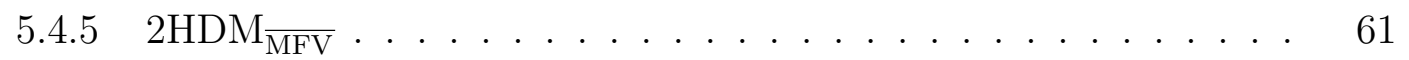

5.4.6 Tree-Level Gauge Boson Exchange . . . . . . . . . . . . 62

5.4.7 Tree-Level Scalar and Pseudoscalar Exchanges . . . . . . . . . 63

5.4.8 Comparison of tree-level $Z^{\prime}$, pseudoscalar and scalar exchanges . 65

5.4.9 Dependence of $\Delta F=1$ Transitions on $M_{Z^{\prime}} \ldots \ldots \ldots \ldots$

5.4.10 Flavour Violating SM $Z$ and SM Higgs Boson . . . . . . . 74

5.4.11 Facing the violation of CMFV Relation (14) . . . . . . 76

5.4.12 $\mathcal{B}\left(B_{s} \rightarrow \mu^{+} \mu^{-}\right)$as an Electroweak Precision Test . . . . 78

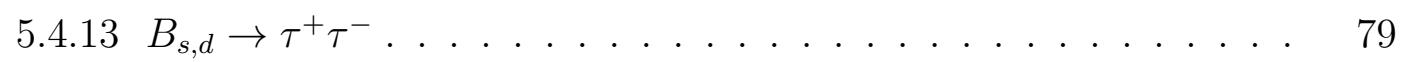

$5.5 \quad$ Step $5: B^{+} \rightarrow \tau^{+} \nu_{\tau} \ldots \ldots \ldots \ldots \ldots \ldots \ldots \ldots$. $\ldots \ldots \ldots$

5.5 .1 Preliminaries . . . . . . . . . . . . . . 79

5.5 .2 Standard Model Results . . . . . . . . . . . . . . . 80

$5.5 .3 \quad \mathrm{CMFV} \ldots \ldots \ldots \ldots \ldots \ldots$

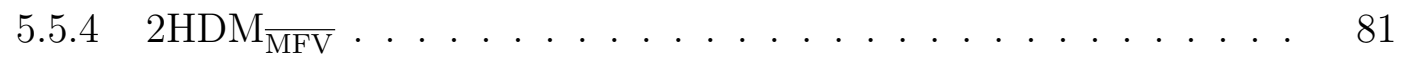

5.5.5 Tree-Level Charged Gauge Boson Exchange . . . . . . . . . 82

5.5.6 Tree-Level Scalar Exchanges . . . . . . . . . . . . . . . . 83

5.6 Step 6: $B \rightarrow X_{s} \gamma$ and $B \rightarrow K^{*} \gamma \ldots \ldots \ldots \ldots$

5.6.1 Standard Model Results . . . . . . . . . . . . . . . . 84

5.6.2 $B \rightarrow X_{s} \gamma$ Beyond the Standard Model . . . . . . . . 8 86

5.7 Step 7: $B \rightarrow X_{s} \ell^{+} \ell^{-}$and $B \rightarrow K^{*}(K) \ell^{+} \ell^{-} \ldots \ldots \ldots \ldots$

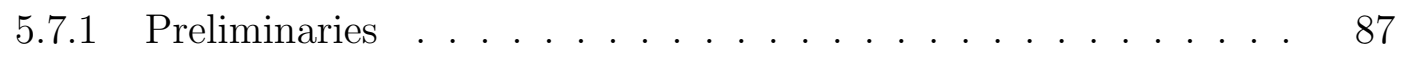

5.7.2 Lessons from Recent Analyses . . . . . . . . . . . . . . . 89

5.7.3 Explicit Bounds on Wilson Coefficients . . . . . . . . . . . 92

5.8 Step 8: $K^{+} \rightarrow \pi^{+} \nu \bar{\nu}, K_{L} \rightarrow \pi^{0} \nu \bar{\nu}$ and $K_{L} \rightarrow \mu^{+} \mu^{-} \ldots \ldots \ldots$

5.8 .1 Preliminaries . . . . . . . . . . . . . . . . . . 93

5.8 .2 Standard Model Results . . . . . . . . . . . . . . . . 97

$5.8 .3 \quad \mathrm{CMFV} \ldots \ldots \ldots \ldots \ldots$ 
$5.8 .42 \mathrm{HDM}_{\overline{\mathrm{MFV}}} \ldots \ldots \ldots \ldots \ldots \ldots$

5.8.5 Tree-Level Gauge Boson Exchanges . . . . . . . . . . . . . 98

5.8.6 Tree-Level Scalar Exchanges . . . . . . . . . . . . . . . . 103

5.9 Step 9: Rare B Decays $B \rightarrow X_{s} \nu \bar{\nu}, B \rightarrow K^{*} \nu \bar{\nu}$ and $B \rightarrow K \nu \bar{\nu} \ldots \ldots$

5.9 .1 Preliminaries . . . . . . . . . . . . . . . . . . . . 104

5.9.2 Standard Model Results . . . . . . . . . . . . . . . . . . 105

$5.9 .3 \quad \mathrm{CMFV} \ldots \ldots \ldots \ldots \ldots \ldots$

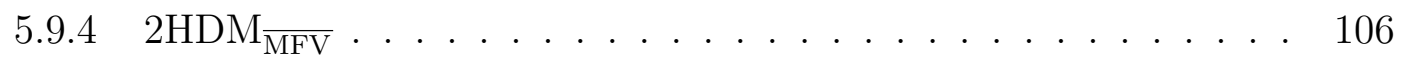

5.9.5 Tree-Level Gauge Boson Exchanges . . . . . . . . . . . . . . 106

5.10 Step 10: The Ratio $\varepsilon^{\prime} / \varepsilon \ldots \ldots$. . . . . . . . . . . . . . 108

5.10 .1 Preliminaries . . . . . . . . . . . . . . . . . . 108

5.10 .2 Basic Formula in the Standard Model . . . . . . . . . . . . . 109

5.11 Step 11: Charm and Top Systems . . . . . . . . . . . . . . . 111

5.11 .1 Preliminaries . . . . . . . . . . . . . . . . . 111

5.11 .2 Charm . . . . . . . . . . . . . . . . . 112

5.11 .3 Top Quark . . . . . . . . . . . . . . . . . . . . . 113

5.12 Step 12: Lepton Flavour Violation, $(g-2)_{\mu, e}$ and EDMs $\ldots \ldots \ldots$

5.12 .1 Preliminaries . . . . . . . . . . . . . . . . . 114

5.12 .2 Charged Lepton Flavour Violation . . . . . . . . . . 115

5.12 .3 Anomalous magnetic moments $(g-2)_{\mu, e} \ldots \ldots \ldots \ldots$

5.12 .4 Electric Dipole Moments (EDMs) . . . . . . . . . . . . 117

6 Towards Selecting Successful Models 119

6.1 Preliminaries . . . . . . . . . . . . . . . . . . . . . . . 119

6.2 DNA-Chart . . . . . . . . . . . . . . . . . 120

6.3 Optimal Observables . . . . . . . . . . . . . . . . . . 121

6.4 Examples of DNA-Charts . . . . . . . . . . . . . . . 122

6.5 Reviewing concrete models . . . . . . . . . . . . . . 124

6.5 .1331 model . . . . . . . . . . . . . . . . . . . . . . 124

6.5.2 Littlest Higgs Model with T-parity . . . . . . . . . . . . 128

6.5.3 The SM with Sequential Fourth Generation (SM4) . . . . . 128 
6.5.4 CP conserving 2HDM II . . . . . . . . . . . . . . 129

6.5.5 Supersymmetric Flavour Models (SF) . . . . . . . . . . . 130

6.5.6 Supersymmetric SO(10) GUT model . . . . . . . . . . . . . . 130

6.5.7 The Minimal Effective Model with Right-handed Currents: RHMFV 131

6.5.8 A Randall-Sundrum Model with Custodial Protection . . . . . . 132

6.5.9 Composite Higgs and Partial Compositeness . . . . . . . . . . . 134

6.5.10 Gauged Flavour Models . . . . . . . . . . . . . 136

6.5.11 New Vectorlike Fermions: a Minimal Theory of Fermion Masses 137

7 Summary and Shopping List 138 


\section{Overture}

The main goal of elementary particle physics is to search for fundamental laws at very short distance scales. From the Heisenberg uncertainty principle we know that to test scales of order $10^{-18} \mathrm{~m}$ we need the energy of approximately $200 \mathrm{GeV}$. Therefore the LHC we will be able to probe distances as short as $4 \cdot 10^{-20} \mathrm{~m}$. Unfortunately, it will take some time before we can reach a higher resolution using high energy processes. On the other hand flavour-violating and CP-violating processes are very strongly suppressed and are governed by quantum fluctuations that allow us to test energy scales as high as $200 \mathrm{TeV}$ corresponding to short distances in the ballpark of $10^{-21} \mathrm{~m}$. Even shorter distance scales can be tested, albeit indirectly, in this manner. Consequently frontiers in testing ultrashort distance scales belong to flavour physics or more concretely to very rare processes like particle-antiparticle mixing, rare decays of mesons, $\mathrm{CP}$ violation and lepton flavour violation. Also electric dipole moments and $(g-2)_{\mu}$ belong to these frontiers even if they are flavour conserving. While such tests are not limited by the available energy, they are limited by the available precision. The latter has to be very high as the Standard Model (SM) has been until now very successful and finding departures from its predictions in the quark sector has become a real challenge. This precision applies both to experiments and theoretical calculations. Among the latter higher order renormalization group improved perturbative QCD calculations and in particular calculations of non-perturbative parameters by means of QCD lattice simulations with dynamical fermions play prominent roles in the search for NP at very short distance scales.

Flavour physics developed over the last two decades into a very broad field. In addition to $K, D$ and $B_{d}$ decays and $K^{0}-\bar{K}^{0}$ and $B_{d}^{0}-\bar{B}_{d}^{0}$ mixings that were with us for quite some time, $B_{s}^{0}-\bar{B}_{s}^{0}$ mixing, $B_{s}$ decays and $D^{0}-\bar{D}^{0}$ mixing belong these days to the standard repertoire of any flavour workshop. Similarly lepton flavour violation (LFV) gained in importance after the discovery of neutrino oscillations and related nonvanishing neutrino masses even if within the SM enriched with tiny neutrino masses LFV is basically unmeasurable. The recent precise measurement of the parameter $\theta_{13}$ resulting in a much higher value than expected by many theorists enhanced the importance of this field. Simultaneously new ideas for the explanation of the quark and lepton mass spectra and the related weak mixings, summarized by the CKM [1,2 and PMNS [3,4] matrices, developed significantly in last two decades. Moreover the analyses of electric dipole moments (EDMs), of the $(g-2)_{\mu}$ anomaly and of flavour changing neutral current (FCNC) processes in top quark decays intensified during the last years in view of the related experimental progress that is expected to take place in this decade.

The correlations between all these observables and the interplay of flavour physics with direct searches for NP and electroweak precision studies will tell us hopefully one day which is the proper extension of the SM. In writing this paper we have been guided by the impressive success of the CKM picture of flavour changing interactions [1,2 
accompanied by the GIM mechanism [5] and also by several tensions between the flavour data and the SM that possibly are the first signals of NP. Fortunately, there is still a lot of room for NP contributions, in particular in rare decays of mesons and charged leptons, in CP-violating transitions and in electric dipole moments of leptons, of the neutron and of other particles. There is also a multitude of models that attempt to explain the existing tensions and to predict what experimentalists should find in this decade.

The main goal of this writing is to have still another look at this fascinating field. However, we should strongly emphasize that we do not intend to present here a review of flavour physics. Comprehensive reviews, written by a hundred of flavour experts are already present on the market [6] and moreover, extensive studies of the physics at future flavour machines and other visions can be found in [9 30].

Even if this overture follows closely the one in [12] and some goals listed there will be encountered below, our presentation is more explicit and is meant as a strategy which we hope we can execute systematically in the coming years. Undoubtedly several ideas presented below appeared already in the literature including those present in our papers. But the collection of these ideas at one place, various correlations between them and in particular new proposals and observations will hopefully facilitate to monitor the coming advances of our experimental colleagues who are searching for the footprints of NP directly at the LHC and indirectly through flavour and CP-violating processes and other rare processes in this decade.

However, in contrast to [12] we will not confine our discussion to scales explored by the ATLAS and CMS but also consider much shorter distance scales.

Our paper is organized as follows. In Section 2 we set the scene for our strategy stressing the importance of correlations between observables. In Section 3 we summarize briefly the theoretical framework for weak decays and briefly present a number of simplest models which will be used to illustrate our ideas. These are in particular models with MFV and models with tree-level FCNCs mediated by neutral gauge bosons and scalars that exhibit transparently non-MFV interactions and the effects of right-handed currents. In Section 4, as a preparation for the subsequent main section of our paper, we present a classification of various correlations between various processes that depend on the NP scenario considered.

Section 5, a very long section, is devoted to the presentation of our strategy that consists of twelve steps and except for Step 12 involves only quark flavour physics. In the course of this presentation we will frequently refer to models of Section 3, illustrating our ideas by means of them. In Section 6 we collect the lessons gained in Section 5 and propose DNA-charts with the goal to transparently exhibit correlations between various observables that are characteristic for a given NP scenario. Finally we briefly review a number of concrete extensions of the SM, investigating how they face the most recent LHCb data. In Section 7 we close this report with a shopping list for this decade. 


\section{Strategy}

\subsection{Setting the Scene}

In order to illustrate the basic spirit of our strategy for the identification of NP through flavour violating processes we recall here a few deviations from SM expectations which could be some signs of NP at work but require further investigations. For non-experts the appearance of several observables not familiar to them already at the start could be some challenge. The detailed table of context should then allow them to quickly find out what a given observable means. In particular various definitions of observables, like $\varepsilon_{K}$ and $S_{\psi K_{S}}$, that are related to $\Delta F=2$ transitions, can be found in Section 5.3, that is in Step 3 of our strategy for the search for NP. It is also a fact that many observables discussed in this review were at the basis of the construction of the SM and appear in the textbooks [31, 32 already, so that the general strategy outlined here should not be difficult to follow. While at first sight the experts could in principle skip this section we would like to ask them not to do it as our strategy for the identification of NP through quark flavour violating processes differs significantly from other strategies found in the literature.

We begin then by recalling a visible tension between the CP-violating observables $\varepsilon_{K}$ and $S_{\psi K_{S}}$ within the SM first emphasized in [33,34]. The nature of this tension depends sensitively on the value of the CKM element $\left|V_{u b}\right|$ for which the exclusive semileptonic decays imply significantly lower value than the inclusive ones. While the latter problem will hopefully be solved in the coming years, it is instructive to consider presently two scenarios for $\left|V_{u b}\right|$ :

- Exclusive (small) $\left|V_{u b}\right|$ Scenario 1: $\left|\varepsilon_{K}\right|$ is smaller than its experimental determination, while $S_{\psi K_{S}}$ is close to its central experimental value.

- Inclusive (large) $\left|V_{u b}\right|$ Scenario 2: $\varepsilon_{K}$ is consistent with its experimental determination, while $S_{\psi K_{S}}$ is significantly higher than its experimental value.

The actual size of discrepancies will be considered in Step 3 of our strategy but the message is clear: dependently which scenario is considered we need either constructive NP contributions to $\left|\varepsilon_{K}\right|$ (Scenario 1) or destructive NP contributions to $S_{\psi K_{S}}$ (Scenario 2). However this NP should not spoil the agreement with the data for $S_{\psi K_{S}}$ (Scenario 1) and for $\left|\varepsilon_{K}\right|$ (Scenario 2).

In view of the fact that the theoretical precision on $S_{\psi K_{S}}$ is significantly larger than in the case of $\varepsilon_{K}$, one may wonder whether removing $1-2 \sigma$ anomaly in $\varepsilon_{K}$ by generating a $2-3 \sigma$ anomaly in $S_{\psi K_{S}}$ is a reasonable strategy. However, we will proceed in this manner as this will teach us how different NP scenarios deal with this problematic. Definitely in order to resolve this puzzle we need not only precise determination of $\left|V_{u b}\right|$ not polluted by NP but also precise values of non-perturbative parameters relevant for $\mathrm{SM}$ predictions in this case. 
Until 2012 there was another significant tension between SM branching ratio for $B^{+} \rightarrow$ $\tau^{+} \nu_{\tau}$ and the data, with the experimental value being by a factor of two larger than the theory. This would favour strongly large $\left|V_{u b}\right|$ scenario. However, presently after the data from BELLE this discrepancy, as discussed in Step 5 of our strategy, is practically absent. Yet, the agreement of the SM with the data still depends on the chosen value of $\left|V_{u b}\right|$ which enters this branching ratio quadratically. In turn the kind of NP which would improve the agreement of the theory with the data depends on the chosen value of $\left|V_{u b}\right|$. Other modest tensions between the SM and the data will be discussed as we proceed.

Now models with many new parameters can face successfully both scenarios for $\left|V_{u b}\right|$ removing the deviations from the data for certain ranges of their parameters but as we will see below in simpler models often only one scenario can be admitted as only in that scenario for $\left|V_{u b}\right|$ a given model has a chance to fit $\varepsilon_{K}$ and $S_{\psi K_{S}}$ simultaneously. For instance as we will see in the course of our presentation models with constrained Minimal Flavour Violation (CMFV) select Scenario 1, while the 2HDM with MFV and flavour blind phases, $2 \mathrm{HDM}_{\overline{\mathrm{MFV}}}$, favours Scenario 2 for $\left|V_{u b}\right|$. What is interesting is that the future precise determination of $\left|V_{u b}\right|$ through tree level decays will be able to distinguish between these two NP scenarios. We will see that there are other models which can be distinguished in this simple manner.

Clearly, in order to get the full picture many more observables have to be considered. For instance in Table 4, that can be found in Step 3, we illustrate the SM predictions for additional observables, in particular the mass differences $\Delta M_{s}$ and $\Delta M_{d}$ in the $B_{s, d}-\bar{B}_{s, d}$ systems. What is striking in this table is that with the present lattice input in Table 1 the predicted central values of $\Delta M_{s}$ and $\Delta M_{d}$ are both in a good agreement with the latter when hadronic uncertainties are taken into account. In particular the central value of the ratio $\Delta M_{s} / \Delta M_{d}$ is very close to the data. These results depend strongly on the lattice input and in the case of $\Delta M_{d}$ on the value of $\gamma$. Therefore to get a better insight both lattice input and the tree level determination of $\gamma$ have to improve. Moreover the situation changes with time. While one year ago lattice input was such that models providing $10 \%$ suppression of both $\Delta M_{s}$ and $\Delta M_{d}$ were favoured, this is no longer the case as can be seen in Table 4.

However, for the purpose of presenting our strategy, it will be useful to keep the old central values from lattice that are consistent within $1 \sigma$ with the present ones but imply certain deviations from SM expectations. This will allow to illustrate how NP can remove these deviations. In doing this we will keep in mind that the pattern of deviations from SM expectations could be modified in the future. This is in particular the case of observables, like $\Delta M_{s, d}$, that still suffer from non-perturbative uncertainties. It could turn out that suppressions (enhancements) of some observables required in our examples from NP will be modified to enhancements (suppressions) in the future and it will be of interest to see whether a given model could cope with such changes. Having this in mind will lead us eventually in Section 6 to a proposal of DNA-charts, primarily with the goal to exhibit transparently the pattern of enhancements and suppressions 
of flavour observables in a given NP scenario and the correlations between them. Of course also this pattern will include situations in which no modifications in a given observable relative to the SM will take place.

\subsection{Towards New Standard Model in 12 Steps}

Our strategy involves twelve steps that we present in detail in Section 5. These steps involve a number of decays and transitions as shown in Fig. 1 and can be properly adjusted in case the pattern of deviations from the SM will be modified.

For the time being assuming that the present tensions will be strengthened with time, when the data improve, the specific questions that arise are:

- Which model is capable of removing the $\varepsilon_{K}-S_{\psi K_{S}}$ tension and simultaneously providing modifications in $B^{+} \rightarrow \tau^{+} \nu_{\tau}$ and $\Delta M_{s, d}$ if they are required?

- What are the predictions of this model for:

$$
\begin{gathered}
S_{\psi \phi}, \quad B_{s, d} \rightarrow \mu^{+} \mu^{-}, \quad B \rightarrow K^{*} \ell^{+} \ell^{-}, \quad B \rightarrow X_{s} \ell^{+} \ell^{-}, \\
B \rightarrow X_{s} \nu \bar{\nu}, \quad, B \rightarrow K^{*} \nu \bar{\nu}, \quad B \rightarrow K \nu \bar{\nu}, \\
K^{+} \rightarrow \pi^{+} \nu \bar{\nu}, \quad K_{L} \rightarrow \pi^{0} \nu \bar{\nu}, \quad \frac{\varepsilon^{\prime}}{\varepsilon}, \quad K_{L} \rightarrow \mu^{+} \mu^{-}
\end{gathered}
$$

and how are these predictions correlated with $S_{\psi K_{S}}$ and $\varepsilon_{K}$ ?

The comparison of processes and observables listed here with those appearing in Fig. 1 should not be understood that the ones missing in (1)-(3), like lepton flavour violation and electric dipole moments, are less important. But as we discuss these topics in our review only in general terms. They will in fact remain under the shadow of the processes listed above.

\subsection{Correlations between Observables}

In order to reach our goal we need a strategy for uncovering new physics responsible for the observed anomalies and possible anomalies hopefully found in the future. One line of attack chosen by several authors are model independent studies of the Wilson coefficients with the goal to find out how much room for NP contributions is still left in each coefficient. In this context correlations between various Wilson coefficients are studied. While such studies are certainly useful and give some insight into the room left for new physics, one should keep in mind that Wilson Coefficients are scale and renormalization scheme dependent and correlations between them generally depend on the scale at which they are evaluated and the renormalization scheme used. 
Therefore it is our strong believe that searching for correlations between the measured observables is more powerful. Extensive studies of correlations between various observables in concrete models illustrate very clearly the power of this strategy. Quite often only a qualitative behaviour of these correlations is sufficient to eliminate the model as a solution to observed anomalies or to select models as candidates for a new Standard Model. A detailed review of such explicit studies can be found in [17,20]. These studies allowed to construct various classifications of NP contributions in the form of "DNA" tables [35 and flavour codes [17] as well as provided some insight into the physics behind resulting correlations in specific models [36]. Detailed analyses in this spirit have been subsequently performed in [37,38. With improved data all these results will be increasingly useful.

In the present paper we will take a slightly different route. Instead of investigating explicit models we will illustrate the search for new Standard Model using very simple models being aware of the fact that in more complicated models certain patterns of flavour violations and correlations between various observables could be washed out and be less transparent. This strategy has been used by us in our most recent papers 39 47]. In this context a prominent role will be played by new tree-level contributions to FCNC processes mediated either by heavy neutral gauge bosons or neutral heavy scalars. These contributions are governed in particular by the couplings $\Delta_{L, R}^{i j}\left(Z^{\prime}\right)$ and $\Delta_{L, R}^{i j}\left(H^{0}\right)$ for gauge bosons and scalars to quarks, respectively. Here $(i, j)$ denote quark flavours. As we will see in addition to a general form of these couplings it will be instructive to consider the following four scenarios for them keeping the pair $(i, j)$ fixed:

1. Left-handed Scenario (LHS) with complex $\Delta_{L}^{b q} \neq 0$ and $\Delta_{R}^{b q}=0$,

2. Right-handed Scenario (RHS) with complex $\Delta_{R}^{b q} \neq 0$ and $\Delta_{L}^{b q}=0$,

3. Left-Right symmetric Scenario (LRS) with complex $\Delta_{L}^{b q}=\Delta_{R}^{b q} \neq 0$,

4. Left-Right asymmetric Scenario (ALRS) with complex $\Delta_{L}^{b q}=-\Delta_{R}^{b q} \neq 0$,

with analogous scenarios for the pair $(s, d)$. These ideas can also be extended to charged gauge boson $\left(W^{\prime}\right)$ and charged Higgs $\left(H^{+}\right)$exchanges. We will see that these simple scenarios will give us a profound insight into the flavour structure of models in which $\mathrm{NP}$ is dominated by left-handed currents or right-handed currents or left-handed and right-handed currents of approximately the same size.

The idea of looking at such NP scenarios is not new and has been in particular motivated by a detailed study of supersymmetric flavour models with NP dominated by LH currents, RH currents or equal amount of LH and RH currents [35]. Moreover, it has been found in several studies of non-supersymmetric frameworks like LHT model [48] or Randall-Sundrum scenario with custodial protection (RSc) [49] that models with the dominance of LH or RH currents exhibit quite different patterns of flavour violation. Our simple models will demonstrate it in a transparent manner. 


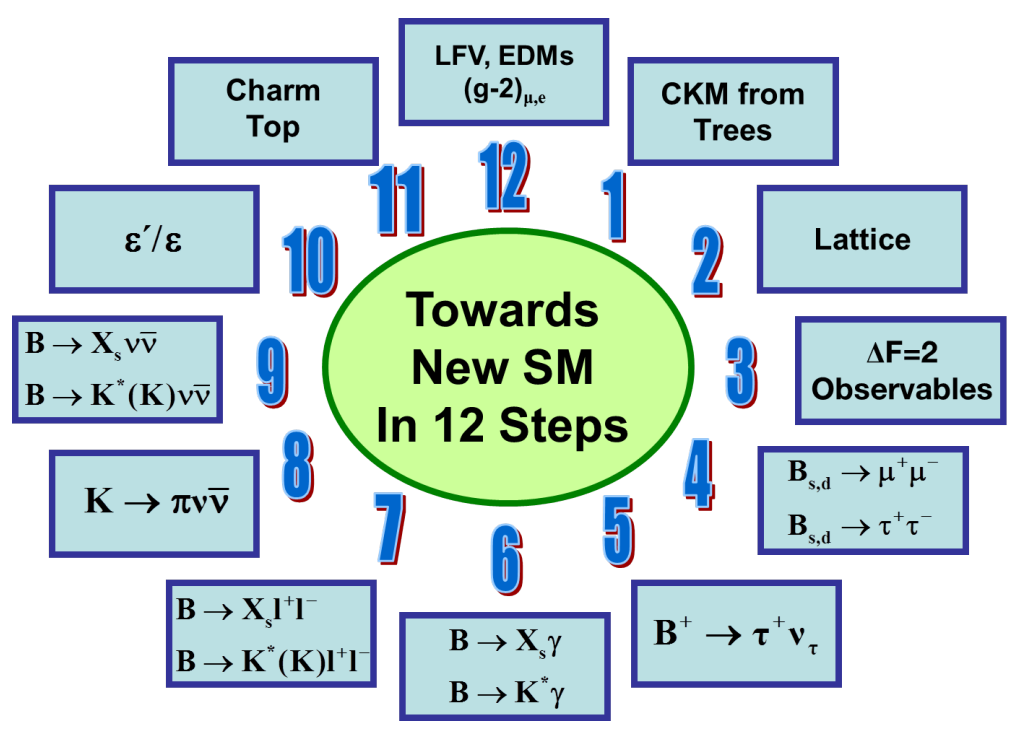

Figure 1: Towards New Standard Model in 12 Steps.

There is another point we would like to make. In several papers predictions for various observables in given extensions of the SM are made using presently available loop processes to determine CKM parameters. As we will emphasize in Step 1 below, in our view this is not the optimal time to proceed in this manner. As last years have shown such predictions have rather a short life-time. It appears to us that it is more useful at this stage to develop transparent formulae which will allow to monitor the future events in flavour physics in the SM and its extensions when the experimental data improve and the uncertainties in lattice calculations decrease.

Our strategy will also be complementary to analyses in which allover fits using sophisticated computer machinery are made. We will start with a subset of observables which have simple theoretical structure ignoring first constraints from more complicated observables. In subsequent steps we will gradually include more observables in our analysis which necessarily will modify our insights gained in the first steps thereby teaching us something. Only in Section 6 we will look at all observables simultaneously and the grand view of simple models and the grand view of more complicated models should hopefully allow us to monitor efficiently flavour events in this decade.

With this general strategy in mind we can now enter the details recalling first briefly the theoretical framework for weak decays. 


\section{Theoretical Framework}

\subsection{Preliminaries}

The field of weak decays is based on effective Hamiltonians with the generic form given as follows

$$
\mathcal{H}^{\text {Prff }} \text { Pross }=\kappa \sum_{i} C_{i}(\mu) Q_{i}+\text { h.c. }
$$

Here $Q_{i}$ are local operators and $C_{i}(\mu)$ their Wilson coefficients that can be evaluated in renormalization group improved perturbation theory. Details on the calculations of these coefficients and the related technology including QCD corrections at the NLO and NNLO level can be found in 5052 .

The overall factor $\kappa$ can be chosen at will in accordance with the overall normalization of Wilson coefficients and operators. Sometimes it is useful to set $\kappa$ to its value in the SM but this is not always the case as we will see below. The scale $\mu$ can be the low energy scale $\mu_{L}$ at which actual lattice calculations are performed or any other scale, in particular the matching scale $\mu_{\mathrm{in}}$, the border line between a given full and corresponding effective theory.

The matrix elements of the effective Hamiltonian are directly related to decay amplitudes and can be written generally as follows:

$$
\left\langle\mathcal{H}_{\text {eff }}^{\text {Process }}\right\rangle=\kappa \sum_{i} C_{i}\left(\mu_{L}\right)\left\langle Q_{i}\left(\mu_{L}\right)\right\rangle
$$

or

$$
\left\langle\mathcal{H}_{\mathrm{eff}}^{\text {Process }}\right\rangle=\kappa \sum_{i} C_{i}\left(\mu_{\mathrm{in}}\right)\left\langle Q_{i}\left(\mu_{\mathrm{in}}\right)\right\rangle .
$$

These two expressions are equal to each other and the Wilson coefficients in them are connected through

$$
\vec{C}\left(\mu_{L}\right)=\hat{U}\left(\mu_{L}, \mu_{\mathrm{in}}\right) \vec{C}\left(\mu_{\mathrm{in}}\right),
$$

where $\hat{U}$ is the renormalization group evolution matrix and $\vec{C}$ a column vector. Which of the formulations is more useful depends on the process and model considered.

Now the Wilson coefficients depend directly on the couplings present in the fundamental theory. In our paper the quark-gauge boson and quark-scalar couplings will play the prominent role and it is useful to introduce a general notation for them so that they can be used in the context of any model considered.

Quite generally we can consider the basic interactions of charged gauge bosons $W^{\prime+}$, charged scalars $H^{+}$, neutral gauge bosons $Z^{\prime}$ and neutral scalars $H^{0}$ with quarks that are shown as vertices in Figs. 2 and 3 . The gauge bosons shown there are all colourless but this notation could be easily extended to coloured gauge bosons and scalars. They can also be extended to heavy quarks interacting with SM quarks and to interactions of 


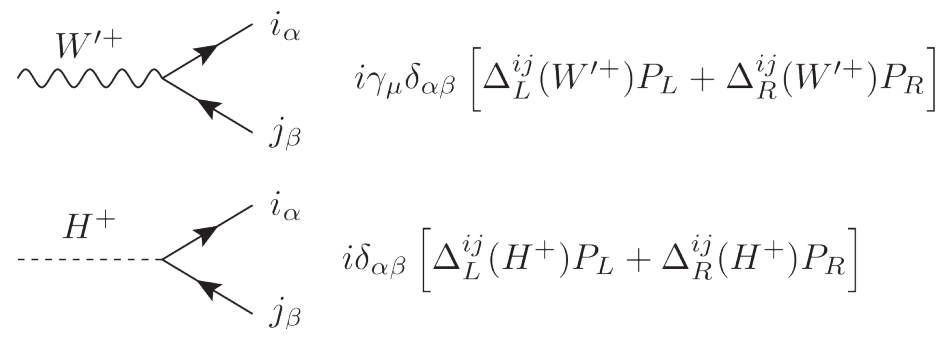

Figure 2: Feynman rules for colourless charged gauge boson $W^{\prime+}$ with mass $M_{W^{\prime}}$, and charged colourless scalar particle $H^{+}$with mass $M_{H}$, where $i(j)$ denotes an up-type (down-type) quark flavour with charge $+\frac{2}{3}\left(-\frac{1}{3}\right)$ and $\alpha, \beta$ are colour indices. $P_{L, R}=$ $\left(1 \mp \gamma_{5}\right) / 2$.

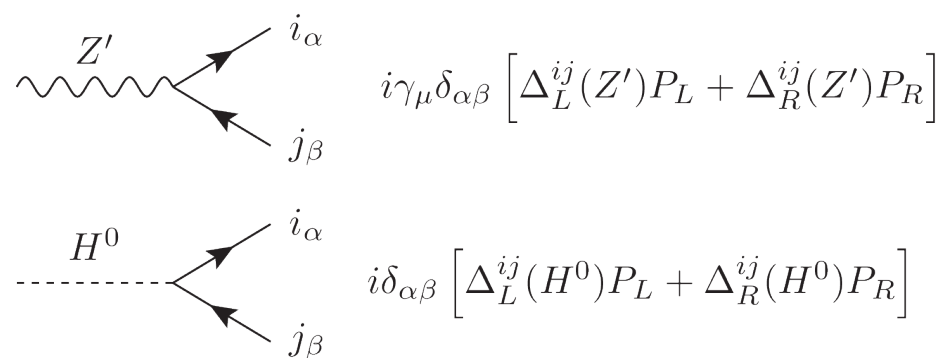

Figure 3: Feynman rules for colourless neutral gauge boson $Z^{\prime}$ with mass $M_{Z^{\prime}}$, and neutral colourless scalar particle $H^{0}$ with mass $M_{H}$, where $i, j$ denote different quark flavours and $\alpha, \beta$ the colours. $P_{L, R}=\left(1 \mp \gamma_{5}\right) / 2$.

bosons with leptons. It should be emphasized that all the fields in these vertices are in the mass eigenstate basis. In the course of our presentation we will give the expressions for various coefficients in terms of these couplings.

In Figs. 2 and 3 the couplings $\Delta_{L, R}$ are $3 \times 3$ complex matrices in the flavour space with $i, j$ denoting different quark flavours. In the case of charged boson exchanges the first flavour index in Fig. 2 denotes an up-type quark and the second a down-type quark.

In models in which FCNC processes take place first at one-loop level, it is useful to work with (6) and express $C_{i}\left(\mu_{\mathrm{in}}\right)$ in terms of a set of gauge independent master functions which result from calculations of penguin and box diagrams and which govern the FCNC processes. In particular this is the case for those models in which the operator structure is the same as in the SM. We will discuss such models soon.

On the other hand in models in which new operators with right-handed currents and scalar and pseudoscalar currents are present, it is necessary to exhibit these new structures explicitly by introducing new loop functions. This is also the case for models with tree-level FCNC processes mediated by gauge bosons and scalars as such exchanges bring in necessarily new operators beyond the ones present in the SM.

We will next introduce a number of simple extensions of the SM that will serve to illustrate our strategy. 


\subsection{Constrained Minimal Flavour Violation (CMFV)}

This is possibly the simplest class of BSM scenarios. It is defined pragmatically as follows [53]:

- The only source of flavour and CP violation is the CKM matrix. This implies that the only $\mathrm{CP}$-violating phase is the $\mathrm{KM}$ phase and that $\mathrm{CP}$-violating flavour blind phases are assumed to be absent.

- The only relevant operators in the effective Hamiltonian below the electroweak scale are the ones present within the SM.

Detailed expositions of phenomenological consequences of this NP scenario has been given in [54,55] and recently in [20].

In CMFV models it is useful to work with (6) and express $C_{i}\left(\mu_{\text {in }}\right)$ in terms of a set of gauge independent master functions which result from calculations of penguin and box diagrams and which govern the FCNC processes. One has then seven one-loop functions that are denoted by ${ }^{1}$

$$
S(v), X(v), Y(v), Z(v), E(v), D^{\prime}(v), E^{\prime}(v),
$$

where the variable $v$ collects the parameters of a given model. It is often useful to keep the CKM factors outside these functions. Then in models with MFV without flavour blind phases these functions are real valued and universal with respect to different meson systems implying various stringent correlations between various decays and related observables. In models with MFV and flavour blind CPV phases and genuine non-MFV frameworks these functions become complex valued and the universality between various meson systems is violated implying corrections to correlations present in models with MFV but no flavour blind phases.

Generally, several master functions contribute to a given decay, although decays exist which depend only on a single function. We have the following correspondence between the most interesting FCNC processes and the master functions in the MFV models in which the operator structure is the same as in the SM:

$$
\begin{array}{ll}
K^{0}-\bar{K}^{0} \text {-mixing }\left(\varepsilon_{K}\right) & S(v) \\
B_{d, s}^{0}-\bar{B}_{d, s}^{0} \text {-mixing }\left(\Delta M_{s, d}\right) & S(v) \\
K \rightarrow \pi \nu \bar{\nu}, B \rightarrow X_{d, s} \nu \bar{\nu} & X(v) \\
K_{\mathrm{L}} \rightarrow \mu \bar{\mu}, B_{d, s} \rightarrow \ell^{+} \ell^{-} & Y(v) \\
K_{\mathrm{L}} \rightarrow \pi^{0} e^{+} e^{-} & Y(v), Z(v), E(v) \\
\varepsilon^{\prime}, \text { Nonleptonic } \Delta B=1, \Delta S=1 & X(v), Y(v), Z(v), E(v) \\
B \rightarrow X_{s} \gamma & D^{\prime}(v), E^{\prime}(v) \\
B \rightarrow X_{s} \text { gluon } & E^{\prime}(v) \\
B \rightarrow X_{s} \ell^{+} \ell^{-} & Y(v), Z(v), E(v), D^{\prime}(v), E^{\prime}(v)
\end{array}
$$

\footnotetext{
${ }^{1}$ The first calculation of these functions within the SM is due to Inami and Lim [56]. The gauge independent form of these functions as used presently in the literature has been introduced in the SM in 57. and in CMFV models in [54.
} 
This table means that the observables like branching ratios, mass differences $\Delta M_{d, s}$ in $B_{d, s}^{0}-\bar{B}_{d, s}^{0}$-mixing and the CP violation parameters $\varepsilon$ and $\varepsilon^{\prime}$, all can be to a very good approximation entirely expressed in terms of the corresponding master functions and the relevant CKM factors.

\subsection{CMFV Relations as Standard Candles of Flavour Physics}

The implications of this framework are so stringent that it appears to us to consider them as standard candles of flavour physics. Even if some of these relations will appear again in the context of our presentation it is useful to collect the most important ones at one place here. A review of these relations is given in [54]. As NP effects in FCNC processes appear smaller than anticipated in the past the importance of these relations increased in 2013.

We have:

1. $S_{\psi K_{S}}$ and $S_{\psi \phi}$ are as in the SM and therefore given by

$$
S_{\psi K_{S}}=\sin (2 \beta), \quad S_{\psi \phi}=\sin \left(2\left|\beta_{s}\right|\right),
$$

where $\beta$ and $\beta_{s}$ are defined in (28).

2. While $\Delta M_{d}$ and $\Delta M_{s}$ can differ from the SM values their ratio is as in the SM

$$
\left(\frac{\Delta M_{d}}{\Delta M_{s}}\right)_{\mathrm{CMFV}}=\left(\frac{\Delta M_{d}}{\Delta M_{s}}\right)_{\mathrm{SM}} .
$$

Moreover, this ratio is given entirely in terms of CKM parameters and non perturbative parameter $\xi$ :

$$
\frac{\Delta M_{d}}{\Delta M_{s}}=\frac{m_{B_{d}}}{m_{B_{s}}} \frac{1}{\xi^{2}}\left|\frac{V_{t d}}{V_{t s}}\right|^{2} r(\Delta M), \quad \xi^{2}=\frac{\hat{B}_{s}}{\hat{B}_{d}} \frac{F_{B_{s}}^{2}}{F_{B_{d}}^{2}}
$$

where we have introduced the quantity $r(\Delta M)$, that is equal unity in models with CMFV. It parametrizes the deviations from these relations found in several models discussed by us below.

3. These two properties allow the construction of the Universal Unitarity Triangle (UUT) of models of CMFV that uses as inputs the measured values of $S_{\psi K_{S}}$ and $\Delta M_{s} / \Delta M_{d}[53]$.

4. The flavour universality of $S(v)$ allows to derive universal expressions for $S_{\psi K_{S}}$ and the angle $\gamma$ in the UUT that depend only on $\left|V_{u s}\right|,\left|V_{c b}\right|$, known from treelevel decays, and non-perturbative parameters entering the evaluation of $\varepsilon_{K}$ and $\Delta M_{s, d}[55,58,59]$. They are valid for all CMFV models. We will present an update of these formulae in Step 3 of our strategy. Therefore, once the data on $\left|V_{u s}\right|,\left|V_{c b}\right|, \varepsilon_{K}$ and $\Delta M_{s, d}$ are taken into account one is able in this framework to predict not only $S_{\psi \phi}$ but also $\left|V_{u b}\right|$. 
5. For fixed CKM parameters determined in tree-level decays, $\left|\varepsilon_{K}\right|, \Delta M_{s}$ and $\Delta M_{d}$, if modified, can only be enhanced relative to SM predictions [60]. Moreover this happens in a correlated manner [59].

6. Two other interesting universal relations in models with CMFV are

$$
\begin{gathered}
\frac{\mathcal{B}\left(B \rightarrow X_{d} \nu \bar{\nu}\right)}{\mathcal{B}\left(B \rightarrow X_{s} \nu \bar{\nu}\right)}=\left|\frac{V_{t d}}{V_{t s}}\right|^{2} r(\nu \bar{\nu}) \\
\frac{\mathcal{B}\left(B_{d} \rightarrow \mu^{+} \mu^{-}\right)}{\mathcal{B}\left(B_{s} \rightarrow \mu^{+} \mu^{-}\right)}=\frac{\tau\left(B_{d}\right)}{\tau\left(B_{s}\right)} \frac{m_{B_{d}}}{m_{B_{s}}} \frac{F_{B_{d}}^{2}}{F_{B_{s}}^{2}}\left|\frac{V_{t d}}{V_{t s}}\right|^{2} r\left(\mu^{+} \mu^{-}\right),
\end{gathered}
$$

where we have again introduced the quantities $r(\nu \bar{\nu})$ and $r\left(\mu^{+} \mu^{-}\right)$that are all equal unity in CMFV models.

7. Eliminating $\left|V_{t d} / V_{t s}\right|$ from (11) and (13) allows to obtain another universal relation within the CMFV models 61

$$
\frac{\mathcal{B}\left(B_{s} \rightarrow \mu^{+} \mu^{-}\right)}{\mathcal{B}\left(B_{d} \rightarrow \mu^{+} \mu^{-}\right)}=\frac{\hat{B}_{d}}{\hat{B}_{s}} \frac{\tau\left(B_{s}\right)}{\tau\left(B_{d}\right)} \frac{\Delta M_{s}}{\Delta M_{d}} r, \quad r=\frac{r(\Delta M)}{r\left(\mu^{+} \mu^{-}\right)}
$$

that does not involve $F_{B_{q}}$ and CKM parameters and consequently contains smaller hadronic and parametric uncertainties than the formulae considered above. It involves only measurable quantities except for the ratio $\hat{B}_{s} / \hat{B}_{d}$ that is now known already from lattice calculations with impressive accuracy of $\pm 2-3 \%[62$ and this precision should be even improved. Therefore the relation (14) should allow a precision test of CMFV even if the branching ratios $\mathcal{B}\left(B_{s, d} \rightarrow \mu^{+} \mu^{-}\right)$would turn out to deviate from SM predictions by $10-20 \%$.

8. All amplitudes for FCNC processes within the CMFV framework can be expressed in terms of seven real and universal master loop functions listed in (8). The implications of this property are numerous correlations between various observables that are discussed more explicitly in Section 4 .

\subsection{Minimal Flavour Violation at Large (MFV)}

In the more general case of MFV the formulation with the help of global symmetries present in the limit of vanishing Yukawa couplings as formulated in [63] is elegant and useful. See also 64 for a similar formulation that goes beyond the MFV. Other profound discussions of various aspects of MFV can be found in [65 70]. An excellent compact formulation of MFV as effective theory has been given by Gino Isidori 71]. We also recommend the reviews in 72, 73, , where phenomenological aspects of MFV are summarized. 
In short the hypothesis of MFV amounts to assuming that the Yukawas are the only sources of the breakdown of flavour and CP-violation. The phenomenological implications of the MFV hypothesis formulated in this more grander manner than the CMFV formulation given above can be found model independently by using an effective field theory approach (EFT) [63]. In this framework the SM Lagrangian is supplemented by all higher dimension operators consistent with the MFV hypothesis, built using the Yukawa couplings as spurion fields. The NP effects in this framework are then parametrized in terms of a few flavour-blind free parameters and SM Yukawa couplings that are solely responsible for flavour violation and also CP violation if these flavourblind parameters are chosen as real quantities as done in [63]. This approach naturally suppresses FCNC processes to the level observed experimentally even in the presence of new particles with masses of a few hundreds GeV. It also implies specific correlations between various observables, which are not as stringent as in the CMFV but are still very powerful.

Yet, it should be stressed that the MFV symmetry principle in itself does not forbid the presence of flavour blind $\mathrm{CP}$ violating sources $[65,67,70,74,78]$ that make effectively the flavour blind free parameters complex quantities having flavour-blind phases (FBPs). These phases can in turn enhance the electric dipole moments (EDMs) of various particles and atoms and in the interplay with the CKM matrix can have also profound impact on flavour violating observables, in particular the $\mathrm{CP}$-violating ones. In the context of the so-called aligned 2HDM model such effects have also been emphasized in [79].

The introduction of flavour-blind CPV phases compatible with the MFV symmetry principle turns out to be a very interesting set-up [65, 67, 69, 70, 77]. In particular, as noted in [69], a large new phase in $B_{s}^{0}-\bar{B}_{s}^{0}$ mixing could in principle be obtained in the MFV framework if additional FBPs are present. This idea cannot be realized in the ordinary MSSM with MFV, as shown in [35,80. The difficulty of realizing this scenario in the MSSM is due to the suppression in the MSSM of effective operators with several Yukawa insertions. Sizable couplings for these operators are necessary both to have an effective large CP-violating phase in $B_{s}^{0}-\bar{B}_{s}^{0}$ mixing and, at the same time, to evade bounds from other observables, such as $B_{s} \rightarrow \mu^{+} \mu^{-}$and $B \rightarrow X_{s} \gamma$. However, it could be realized in different underlying models, such as the up-lifted MSSM, as pointed out in [81], in the so-called beyond-MSSM scenarios 82, 83] and in the 2HDM with MFV and FBPs, the so-called $2 \mathrm{HDM}_{\overline{\mathrm{MFV}}}[84$ to which we will return at various places in this writing. An excellent review of $2 \mathrm{HDM}$ s at large can be found in [85].

As we will see in Step 3 of our strategy the present data from the LHCb show that the new phases in $B_{s}^{0}-\bar{B}_{s}^{0}$ mixing, if present, must be rather small. Consequently also the role of flavour blind phases in describing data decreased significantly relatively to the one they played in the studies summarized above. However, the full assessment of the importance of these phases will only be possible when the CP-violation in $B_{s}^{0}$ $\bar{B}_{s}^{0}$ mixing will be precisely measured and also the bounds on electric dipole moments improve. 


\subsection{Simplest Models with non-MFV Sources}

In models with new sources of flavour and $\mathrm{CP}$ violation in which the operator structure is not modified, the formulation of FCNC processes in terms of seven one-loop functions is useful as well but when the CKM factors are the only ones kept explicit as overall factors, these functions become complex and are different for different meson systems. We have then $(i=K, d, s)$ :

$$
\begin{gathered}
S_{i} \equiv\left|S_{i}\right| e^{i \theta_{S}^{i}}, \quad X_{i} \equiv\left|X_{i}\right| e^{i \theta_{X}^{i}}, \quad Y_{i} \equiv\left|Y_{i}\right| e^{i \theta_{Y}^{i}}, \quad Z_{i} \equiv\left|Z_{i}\right| e^{i \theta_{Z}^{i}} \\
E_{i} \equiv\left|E_{i}\right| e^{i \theta_{E}^{i}}, \quad D_{i}^{\prime} \equiv\left|D_{i}^{\prime}\right| e^{i \theta_{D^{\prime}}^{i}}, \quad E_{i}^{\prime} \equiv\left|E_{i}^{\prime}\right| e^{i \theta_{E^{\prime}}^{i}} .
\end{gathered}
$$

As now the property of the universality of these functions is lost, the usual CMFV relations between $K, B_{d}$ and $B_{s}$ systems listed above can be violated and the parameters $r(k)$ introduced in the context of our discussion of CMFV models are generally different from unity and can be complex. A known example is the Littlest Higgs Model with T-parity (LHT) [48].

\subsection{The $U(2)^{3}$ Models}

Probably the simplest models with new sources of flavour violation are models in which the $U(3)^{3}$ symmetry of MFV models is reduced to $U(2)^{3}$ symmetry 86 92. As pointed out in [39] a number of properties of CMFV models remains in this class of models, in particular the relation (14) is still valid. On the other hand there are profound differences due to the presence of new CP-phases which we will discuss in the course of our presentation.

\subsection{Tree-Level Gauge Boson and Scalar Exchanges}

In a number of BSM scenarios NP can enter already at tree level, both in charged current processes and in FCNC processes.

In the case of charged current processes prominent examples are the right-handed $W^{ \pm \prime}$ bosons in left-right symmetric models and charged Higgs $\left(H^{ \pm}\right)$particles in models with extended scalar sector like two Higgs doublet models and supersymmetric models. In these models new operators are present, the simplest example being $(V+A) \times(V+A)$ operators originating in the exchange of $W^{ \pm \prime}$ gauge bosons in the left-right symmetric models. In these models also $(V-A) \times(V+A)$ operators contribute. These operators generate in turn through QCD corrections $(S-P) \times(S+P)$ operators present also in models with $H^{ \pm}$particles. In the latter models also $(S \pm P) \times(S \pm P)$ operators are present. Needless to say all these statements also apply to neutral gauge bosons 
and scalars mediating $\Delta F=1$ transitions. It should also be stressed that anomalous right-handed couplings of SM gauge bosons $W^{ \pm}$to quarks can be generated through the mixing with heavy vectorial fermions.

Concerning FCNC processes, tree-level transitions are present in any model in which GIM mechanism is absent in some sectors of a given model. This is the case of numerous $Z^{\prime}$ models, gauged flavour models with new very heavy neutral gauge bosons and LeftRight symmetric models with heavy neutral scalars. They can also be generated at one loop in models having GIM at the fundamental level and Minimal Flavour Violation of which Two-Higgs Doublet models with and without supersymmetry are the best known examples. Tree-level $Z^{0}$ and SM neutral Higgs $H^{0}$ contributions to $\Delta F=2$ processes are also possible in models containing vectorial heavy fermions that mix with the standard chiral quarks. This is also the case of models in which $Z^{0}$ and SM neutral Higgs $H^{0}$ mix with new heavy gauge bosons and scalars in the process of electroweak symmetry breaking. Recently two very detailed analyses of FCNCs within models with tree-level gauge boson and neutral scalar and pseudoscalar exchanges have been performed in 40,44 and we will include the highlights from these two papers in our discussion.

In the previous section we defined in Figs. 2 and 3 the basic interactions of charged gauge bosons $W^{\prime+}$, charged scalars $H^{+}$, with quarks. In the flavour precision era also QCD corrections to tree-level exchanges have to be taken into account. They depend on whether a gauge boson or scalar is exchanged and of course on the process considered. Fortunately, the NLO matching conditions for tree-level neutral gauge bosons $Z^{\prime}$ and neutral scalars $H^{0}$ exchanges have been calculated recently in [93,94]. Combining them with previously calculated two-loop anomalous dimensions of four-quark operators, it is possible to perform complete NLO renormalization group analysis in this case.

Finally, we would like to make a general comment on the expressions for various observables in this class of models that we will encounter below. They are very general and apply also to models in which the FCNC processes enter first at the one-loop level. Indeed they contain very general operator structure and general new flavour violating and CP-violating interactions. However, having simpler coupling structure than in the case of models in which NP is dominated by loop contributions, allows us to have an analytic look at various correlations between various observables as we will see below.

\section{Classifying Correlations between various Observ- ables}

As we have seen in preceding sections, in the SM and in models with CMFV the observables measured in the processes shown in Fig. 1 depend on selected number of basic universal functions that are the same for $K$ and $B_{s, d}$ decays. In particular $\Delta F=2$ processes depend only on the function $S(v)$, while the most important rare $K$ 
and $B_{s, d}$ decays depend on three universal functions $X(v), Y(v), Z(v)$. Consequently, a number of correlations exist between various observables not only within the $K$ and $B$ systems but also between $K$ and $B$ systems. In particular the latter correlations are very interesting as they are characteristic for this class of models. A review of these correlations is given in [54]. These correlations are violated in several extensions of the SM either through the presence of new source of flavour violation or the presence of new operators. However, as the SM constitutes the main bulk of most branching ratios, the CMFV correlations can be considered as standard candles of flavour physics with help of which new sources of flavour violation or effects of new operators could be identified. It is for the latter reason that we prefer to use CMFV correlations as standard flavour candles and not those present in MFV at large, but models with MFV and one Higgs doublet give the same results.

In [49] a classification of correlations following from CMFV has been presented. In what follows we will somewhat modify this classification so that it fits better to our presentation in the next section that considers a number of models in contrast to [49] where only the RSc model has been studied.

We distinguish the following classes of correlations in CMFV model:2?

\section{Class 1:}

Correlations implied by the universality of the real function $X$. They involve rare $K$ and $B$ decays with $\nu \bar{\nu}$ in the final state. These are:

$$
K^{+} \rightarrow \pi^{+} \nu \bar{\nu}, \quad K_{L} \rightarrow \pi^{0} \nu \bar{\nu}, \quad B \rightarrow X_{s, d} \nu \bar{\nu}, \quad B \rightarrow K^{*}(K) \nu \bar{\nu} .
$$

\section{Class 2:}

Correlations implied by the universality of the real function $Y$. They involve rare $K$ and $B$ decays with $\mu^{+} \mu^{-}$in the final state. These are

$$
B_{s, d} \rightarrow \mu^{+} \mu^{-}, \quad K_{L} \rightarrow \mu^{+} \mu^{-}, \quad K_{L} \rightarrow \pi^{0} \mu^{+} \mu^{-}, \quad K_{L} \rightarrow \pi^{0} e^{+} e^{-} .
$$

\section{Class 3:}

In models with CMFV NP contributions enter the functions $X$ and $Y$ approximately in the same manner as at least in the Feynman gauge they come dominantly from $Z^{0}$-penguin diagrams. This implies correlations between rare decays with $\mu^{+} \mu^{-}$and $\nu \bar{\nu}$ in the final state. It should be emphasized that this is a separate class as NP can generally have a different impact on decays with $\nu \bar{\nu}$ and $\mu^{+} \mu^{-}$in the final state. This class involves simply the decays of Class 1 and Class 2.

\section{Class 4:}

\footnotetext{
${ }^{2}$ In this list we do not include a known model independent correlation between the asymmetries $S_{\psi \phi}$ and $A_{\mathrm{SL}}^{s} 95$ that has to be satisfied basically in any extension of the SM.
} 
Here we group correlations between $\Delta F=2$ and $\Delta F=1$ transitions in which the one-loop functions $S$ and $(X, Y)$, respectively, cancel out and the correlations follow from the fact that the CKM parameters extracted from tree-level decays are universal. One known correlation of this type involves [96, 97

$$
K^{+} \rightarrow \pi^{+} \nu \bar{\nu}, \quad K_{L} \rightarrow \pi^{0} \nu \bar{\nu} \quad \text { and } \quad S_{\psi K_{S}},
$$

another one 61

$$
B_{s, d} \rightarrow \mu^{+} \mu^{-} \quad \text { and } \quad \Delta M_{s, d} .
$$

As we will see in Section 5, some of these correlations, in particular those between $K$ and $B$ decays are strongly violated in certain models, others are approximately satisfied. Clearly the full picture is only obtained by looking simultaneously at patterns of violations of the correlations in question in a given NP scenario.

At later stages of our presentation in Section 5 we will study correlations in models with tree-level FCNCs mediated by neutral gauge bosons and scalars that go beyond the CMFV framework. In these models multi-correlations between various observables in a given meson system are predicted and it is useful to group these processes in the following three classes. These are:

\section{Class 5:}

$$
\varepsilon_{K}, \quad K^{+} \rightarrow \pi^{+} \nu \bar{\nu}, \quad K_{L} \rightarrow \pi^{0} \nu \bar{\nu}, \quad K_{L} \rightarrow \mu^{+} \mu^{-}, \quad K_{L} \rightarrow \pi^{0} \ell^{+} \ell^{-}, \quad \varepsilon^{\prime} / \varepsilon .
$$

\section{Class 6:}

$$
\Delta M_{d}, \quad S_{\psi K_{S}}, \quad B_{d} \rightarrow \mu^{+} \mu^{-}, \quad S_{\mu \mu}^{d},
$$

where the CP-violating asymmetry $S_{\mu \mu}^{d}$ can only be obtained from time-dependent rate of $B_{d} \rightarrow \mu^{+} \mu^{-}$and will remain in the realm of theory for the foreseeable future.

\section{Class 7:}

$$
\Delta M_{s}, \quad S_{\psi \phi}, \quad B_{s} \rightarrow \mu^{+} \mu^{-}, \quad S_{\mu \mu}^{s}, \quad B \rightarrow K \nu \bar{\nu}, \quad B \rightarrow K^{*} \nu \bar{\nu}, \quad B \rightarrow X_{s} \nu \bar{\nu},
$$

where the measurement of $S_{\mu \mu}^{s}$ will require heroic efforts from experimentalists but apparently is not totally hopeless.

\section{Class 8:}

$$
B \rightarrow X_{s} \gamma, \quad B \rightarrow K^{*} \gamma, \quad B^{+} \rightarrow \tau^{+} \nu_{\tau}
$$

in which new charged gauge bosons and heavy scalars can play significant role. The first two differ from previous decays as they are governed by dipole operators. 


\section{Class 9}

$$
B \rightarrow K \mu^{+} \mu^{-}, \quad B \rightarrow K^{*} \mu^{+} \mu^{-}, \quad B \rightarrow X_{s} \mu^{+} \mu^{-}
$$

to which several operators contribute and for which multitude of observables can be defined. Moreover in the case of FCNCs mediated by tree-level neutral gauge boson exchanges interesting correlations between these observables and the ones of Class 7 exist.

\section{Class 10:}

Correlations between $K$ and $D$ observables.

\section{Class 11:}

Correlations between quark flavour violation, lepton flavour violation, electric dipole moments and $(g-2)_{e, \mu}$.

\section{$5 \quad$ Searching for New Physics in twelve Steps}

\subsection{Step 1: The CKM Matrix from tree level decays}

As the SM represents already the dominant part in very many flavour observables it is crucial to determine the CKM parameters as precise as possible independently of NP contributions. Here the tree-level decays governed by $W^{ \pm}$exchanges play the prominent role. The charged current decays could be affected by heavy charged new gauge boson exchanges and heavy charged Higgs boson exchanges that could contribute directly to tree level decays. Also non-standard $W^{ \pm}$couplings could be generated through mixing of $W^{ \pm}$with the new heavy gauge bosons in the process of electroweak symmetry breaking. Moreover, the mixing of heavy fermions, both sequential like the case of fourth generation or vectorial ones present in various NP scenarios, could make the CKM matrix to be non-unitary not allowing to use the well known unitarity relations of this matrix. This mixing would also generate non-standard $W^{ \pm}$couplings to SM quarks.

The non-observation of any convincing NP signals at the LHC until now gives some hints that the masses of new charged particles are shifted above the $500 \mathrm{GeV}$ scale. Therefore NP effects in charged current decays are likely to be at most at the level of a few percent. While effects of this sort could play a role one day, it is a good strategy to assume in the first step that tree level charged current decays are fully dominated by $W^{ \pm}$exchanges with SM couplings and consequently by the CKM matrix.

The goal of this first step is then a very precise determination of

$$
\left|V_{u s}\right| \simeq s_{12}, \quad\left|V_{u b}\right| \simeq s_{13}, \quad\left|V_{c b}\right| \simeq s_{23}, \quad \gamma=\delta,
$$




\begin{tabular}{|c|c|c|c|}
\hline$G_{F}=1.16637(1) \times 10^{-5} \mathrm{GeV}^{-2}$ & 102 & $m_{B_{d}}=m_{B^{+}}=5279.2(2) \mathrm{MeV}$ & 103 \\
\hline$M_{W}=80.385(15) \mathrm{GeV}$ & 102 & $m_{B_{s}}=5366.8(2) \mathrm{MeV}$ & $\overline{03}$ \\
\hline $\sin ^{2} \theta_{W}=0.23116(13)$ & 102 & $F_{B_{d}}=(190.5 \pm 4.2) \mathrm{MeV}$ & $\overline{04}$ \\
\hline$\alpha\left(M_{Z}\right)=1 / 127.9$ & 102 & $F_{B_{s}}=(227.7 \pm 4.5) \mathrm{MeV}$ & 4 \\
\hline$\alpha_{s}\left(M_{Z}\right)=0.1184(7)$ & 102 & $F_{B^{+}}=(185 \pm 3) \mathrm{MeV}$ & \\
\hline$m_{u}(2 \mathrm{GeV})=2.16(11) \mathrm{MeV}$ & 104 & $\hat{B}_{B_{d}}=1.27(10), \hat{B}_{B_{s}}=1.33(6)$ & 4 \\
\hline$m_{d}(2 \mathrm{GeV})=4.68(0.15) \mathrm{MeV}$ & 104 & $\hat{B}_{B_{s}} / \hat{B}_{B_{d}}=1.01(2)$ & 2 \\
\hline$m_{s}(2 \mathrm{GeV})=93.8(24) \mathrm{MeV}$ & 104 & $F_{B_{d}} \sqrt{\hat{B}_{B_{d}}}=216(15) \mathrm{MeV}$ & 101 \\
\hline$m_{c}\left(m_{c}\right)=(1.279 \pm 0.013) \mathrm{GeV}$ & 106 & $F_{B_{s}} \sqrt{\hat{B}_{B_{s}}}=266(18) \mathrm{MeV}$ & \\
\hline$m_{b}\left(m_{b}\right)=4.19_{-0.06}^{+0.18} \mathrm{GeV}$ & 102 & $\xi=1.268(63)$ & 4 \\
\hline$m_{t}\left(m_{t}\right)=163(1) \mathrm{GeV}$ & 108 & $\eta_{B}=0.55(1)$ & 0 \\
\hline$M_{t}=173.2 \pm 0.9 \mathrm{GeV}$ & 111 & $\Delta M_{d}=0.507(4) \mathrm{ps}^{-1}$ & 12 \\
\hline$m_{K}=497.614(24) \mathrm{MeV}$ & 102 & $\Delta M_{s}=17.72(4) \mathrm{ps}^{-1}$ & 12 \\
\hline$F_{K}=156.1(11) \mathrm{MeV}$ & 107 & $S_{\psi K_{S}}=0.68(2)$ & 12 \\
\hline$\hat{B}_{K}=0.766(10)$ & 104 & $S_{\psi \phi}=0.00(7)$ & 2 \\
\hline$\kappa_{\epsilon}=0.94(2)$ & 113 & $\Delta \Gamma_{s} / \Gamma_{s}=0.123(17)$ & 12 \\
\hline$\eta_{c c}=1.87(76)$ & 114 & $\tau_{B_{s}}=1.509(11) \mathrm{ps}$ & 12 \\
\hline$\eta_{t t}=0.5765(65)$ & 109 & $\tau_{B_{d}}=1.519(7) \mathrm{ps}$ & $\overline{\overline{2}}$ \\
\hline$\eta_{c t}=0.496(47)$ & 115 & $\tau_{B^{ \pm}}=1.642(8) \mathrm{ps}$ & 12 \\
\hline$\Delta M_{K}=0.5292(9) \times 10^{-2} \mathrm{ps}^{-1}$ & 102 & $\left|V_{u s}\right|=0.2252(9)$ & 12 \\
\hline$\left|\varepsilon_{K}\right|=2.228(11) \times 10^{-3}$ & 102 & $\left|V_{c b}\right|=(40.9 \pm 1.1) \times 10^{-3}$ & 03 \\
\hline$\left|V_{c b}^{\text {incl. }}\right|=42.4(9) \times 10^{-3}$ & 116 & $\left|V_{u b}^{\text {incl. }}\right|=4.40(25) \times 10^{-3}$ & 04 \\
\hline$\left|V_{c b}^{\text {excl. }}\right|=39.4(6) \times 10^{-3}$ & 104 & $\left|V_{u b}^{\text {excl. }}\right|=3.42(31) \times 10^{-3}$ & 04 \\
\hline
\end{tabular}

Table 1: Values of the experimental and theoretical quantities used as input parameters as of April 2014. For future updates see PDG [103], FLAG [104] and HFAG [112].

where on the l.h.s we give the measured quantities and on the r.h.s the determined parameters of the CKM matrix given in the standard parametrization:

$$
\hat{V}_{\mathrm{CKM}}=\left(\begin{array}{ccc}
c_{12} c_{13} & s_{12} c_{13} & s_{13} e^{-i \delta} \\
-s_{12} c_{23}-c_{12} s_{23} s_{13} e^{i \delta} & c_{12} c_{23}-s_{12} s_{23} s_{13} e^{i \delta} & s_{23} c_{13} \\
s_{12} s_{23}-c_{12} c_{23} s_{13} e^{i \delta} & -s_{23} c_{12}-s_{12} c_{23} s_{13} e^{i \delta} & c_{23} c_{13}
\end{array}\right) .
$$

The phase $\gamma$ is one of the angles of the unitarity triangle shown Fig. 4. We emphasize that the relations in (26) are excellent approximations. Indeed $c_{13}$ and $c_{23}$ are very close to unity. The parameters $\bar{\varrho}$ and $\bar{\eta}$ are the generalized Wolfenstein parameters $[58,98]$. Extensive analyses of the unitarity triangle have been performed for many years by CKMfitter [99] and UTfit [100] collaborations and recently by SCAN-Method collaboration 101.

Under the assumption made above this determination would give us the values of the elements of the CKM matrix without NP pollution. From the present perspective most 
important are the determinations of $\left|V_{u b}\right|$ and $\gamma$ because as seen in Table 1 they are presently not as well known as $\left|V_{c b}\right|$ and $\left|V_{u s}\right|$. In this table we give other most recent values of the relevant parameters to which we will return in the course of our review.

Looking at Table 1 we make the following observations:

- The element $\left|V_{u s}\right|$ is already well measured.

- The accuracy of the determination of $\left|V_{c b}\right|$ is quite good but disturbing is the discrepancy between the inclusive and exclusive determinations [116, 117], with the exclusive ones being visibly smaller [118. We quote here only the average value provided by PDG. It should be recalled that the knowledge of this CKM matrix element is very important for rare decays and $\mathrm{CP}$ violation in the $K$ meson system. Indeed $\varepsilon_{K}, \mathcal{B}\left(K^{+} \rightarrow \pi^{+} \nu \bar{\nu}\right)$ and $\mathcal{B}\left(K_{L} \rightarrow \pi^{0} \nu \bar{\nu}\right)$ are all roughly proportional to $\left|V_{c b}\right|^{4}$ and even a respectable accuracy of $2 \%$ in $\left|V_{c b}\right|$ translates into $8 \%$ parametric uncertainty in these observables. This is in particular disturbing for $\mathcal{B}\left(K^{+} \rightarrow \pi^{+} \nu \bar{\nu}\right)$ and $\mathcal{B}\left(K_{L} \rightarrow \pi^{0} \nu \bar{\nu}\right)$ as these branching ratios are practically independent of any theoretical uncertainties. Future $B$-facilities accompanied by improved theory should be able to determine $\left|V_{c b}\right|$ with precision of $1-2 \%$.

- The case of $\left|V_{u b}\right|$ is more disturbing with central values from inclusive determinations being by roughly $25 \%$ higher than the corresponding value resulting from exclusive semi-leptonic decays. We will see below that dependently on which of these values is assumed, different conclusions on the properties of NP responsible for certain anomalies seen in the data will be reached. Again, future $B$-facilities accompanied by improved theory should be able to determine $\left|V_{u b}\right|$ with precision of $1-2 \%$.

- Finally, the only physical CP phase in the CKM matrix, $\gamma$, is still poorly known from tree-level decays. But LHCb should be able to determine this angle with an error of a few degrees, which would be a great achievement. Further improvements could come from SuperKEKB.

The importance of precise determinations of $\left|V_{c b}\right|,\left|V_{u b}\right|$ and $\gamma$ should not be underestimated. Table 3 and Fig 2 in [119] showing SM predictions for various combinations of $\left|V_{c b}\right|$ and $\left|V_{u b}\right|$ demonstrate this very clearly. Therefore the consequences of reaching our first goal would be profound. Indeed, having determined precisely the four parameters of the CKM matrix without influence from NP, will allow us to reconstruct all its elements. In turn they could be used efficiently in the calculation of the SM predictions for all decays and in particular FCNC processes, both CP-conserving and CP-violating. Moreover, this would allow to calculate not only an important element $\left|V_{t d}\right|$ but also its phase $-\beta$, with $\beta$ denoting another, very important angle of the unitarity triangle in Fig. 4. 


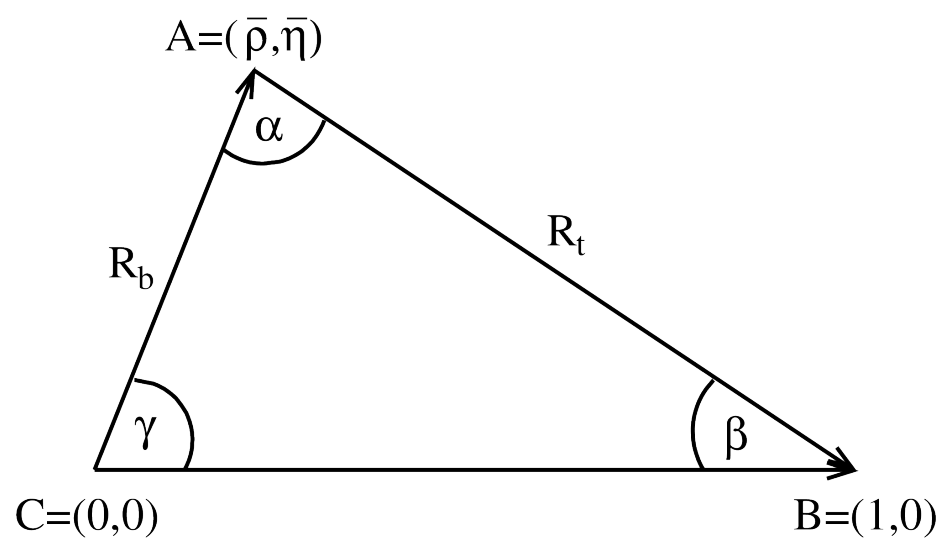

Figure 4: Unitarity Triangle.

In order to be prepared for these developments we collect here the most important formulae related to the unitarity triangle and CKM matrix. The phases of $V_{t d}$ and $V_{t s}$ are defined by

$$
V_{t d}=\left|V_{t d}\right| e^{-i \beta}, \quad V_{t s}=-\left|V_{t s}\right| e^{-i \beta_{s}} .
$$

Next, the lengths $C A$ and $B A$ in the unitarity triangle are given respectively by

$$
\begin{gathered}
R_{b} \equiv \frac{\left|V_{u d} V_{u b}^{*}\right|}{\left|V_{c d} V_{c b}^{*}\right|}=\sqrt{\bar{\varrho}^{2}+\bar{\eta}^{2}}=\left(1-\frac{\lambda^{2}}{2}\right) \frac{1}{\lambda}\left|\frac{V_{u b}}{V_{c b}}\right|, \\
R_{t} \equiv \frac{\left|V_{t d} V_{t b}^{*}\right|}{\left|V_{c d} V_{c b}^{*}\right|}=\sqrt{(1-\bar{\varrho})^{2}+\bar{\eta}^{2}}=\frac{1}{\lambda}\left|\frac{V_{t d}}{V_{c b}}\right| .
\end{gathered}
$$

An important very accurate relation is

$$
\sin 2 \beta=2 \frac{\bar{\eta}(1-\bar{\varrho})}{R_{t}^{2}} .
$$

We also note that the knowledge of $\left(R_{b}, \gamma\right)$ from tree-level decays gives

$$
\left|V_{t d}\right|=\left|V_{u s}\right|\left|V_{c b}\right| R_{t}, \quad R_{t}=\sqrt{1+R_{b}^{2}-2 R_{b} \cos \gamma}, \quad \cot \beta=\frac{1-R_{b} \cos \gamma}{R_{b} \sin \gamma} .
$$

Similarly the knowledge of $\left(R_{t}, \beta\right)$ allows to determine $\left(R_{b}, \gamma\right)$ through

$$
R_{b}=\sqrt{1+R_{t}^{2}-2 R_{t} \cos \beta}, \quad \cot \gamma=\frac{1-R_{t} \cos \beta}{R_{t} \sin \beta}
$$

and consequently with known $\lambda=\left|V_{u s}\right|$ and $\left|V_{c b}\right|$, one finds $\left|V_{u b}\right|$ by means of (29). Similarly $V_{t s}$ can be calculated. $\left|V_{t s}\right|$ is slightly below $\left|V_{c b}\right|$ but in the flavour precision 
era it is better to calculate its value numerically by using the standard parametrization. Then one also finds that the value of $\beta_{s}$ is tiny: $\beta_{s} \approx-1^{\circ}$.

There is still another powerful route to the determination of the Unitarity Triangle. As pointed out in [120] in addition to the determination of UT without any NP pollution through the determination of $\left(R_{b}, \gamma\right)$, in models with CMFV and MFV in which NP is absent in $S_{\psi K_{S}}$, the determination can proceed through $(\beta, \gamma)$. Then

$$
R_{t}=\frac{\sin \gamma}{\sin (\beta+\gamma)}, \quad R_{b}=\frac{\sin \beta}{\sin (\beta+\gamma)} .
$$

In fact as demonstrated in [120] $\left(R_{b}, \gamma\right)$ and $(\beta, \gamma)$ are the two most powerful ways to determine UT in the sense that the accuracy on these two pairs does not have to be very high in order to determine $(\bar{\varrho}, \bar{\eta})$ with good precision. But as we have seen $\left|V_{u b}\right|$ is not known very well and even if there are hopes to determine it within few $\%$ in the second half of this decade, it is more probable that $\gamma$ from tree-level decays will be known with this accuracy first and the $(\beta, \gamma)$ strategy will be leading one in getting $(\bar{\varrho}, \bar{\eta})$ within $\mathrm{CMFV}$ and MFV models.

The values of $\left|V_{t d}\right|$ and $\left|V_{t s}\right|$ are crucial for the predictions of various rare decays but in particular for the mass differences $\Delta M_{d}$ and $\Delta M_{s}$ and the phases $\beta$ and $\beta_{s}$ for the corresponding mixing induced CP-asymmetries $S_{\psi K_{S}}$ and $S_{\psi \phi}$, which are defined within the SM in (52). Also the CP-violating parameter $\varepsilon_{K}$ depends crucially on $V_{t d}$ and $V_{t s}$.

Before making some statements about the present status of the first five super stars of flavour physics

$$
\Delta M_{d}, \quad \Delta M_{s}, \quad S_{\psi K_{S}}, \quad S_{\psi \phi}, \quad \varepsilon_{K}
$$

within the SM, we have to make the second very important step.

\subsection{Step 2: Improved Lattice Calculations of Hadronic Pa- rameters}

Precise knowledge of the meson decay constants $F_{B_{s}}, F_{B_{d}}, F_{B^{+}}$and of various nonperturbative parameters $B_{i}$ related to hadronic matrix elements of SM operators and operators found in the extensions of the SM is very important. Indeed this would allow in conjunction with Step 1 to perform precise calculations of $\Delta M_{s}, \Delta M_{d}, \varepsilon_{K}$, $\mathcal{B}\left(B_{s, d} \rightarrow \mu^{+} \mu^{-}\right), \mathcal{B}\left(B^{+} \rightarrow \tau^{+} \nu_{\tau}\right)$ and of other observables in the SM. We could then directly see whether the SM is capable of describing these observables or not. The recent unquenched lattice calculations allow for optimism and in fact a very significant progress in the calculation of $\hat{B}_{K}$, that is relevant for $\varepsilon_{K}$, has been made recently. Also the weak decay constants $F_{B_{s}}, F_{B_{d}}$ and $F_{B^{+}}$and some non-perturbative $B_{i}$ parameters are much better known than few years ago.

In Table 1 we collect most relevant non-perturbative parameters relevant for $\Delta F=2$ observables that we extracted from the most recent FLAG average [104]. It should 
be remarked that these values are consistent with the ones presented in [105, 107] but generally have larger errors as FLAG prefers to be conservative. In particular in the latter two papers one finds:

$$
\begin{gathered}
F_{B_{s}} \sqrt{\hat{B}_{B_{s}}}=279(13) \mathrm{MeV}, \quad F_{B_{d}} \sqrt{\hat{B}_{B_{d}}}=226(13) \mathrm{MeV}, \quad \xi=1.237(32), \\
F_{B_{s}}=225(3) \mathrm{MeV}, \quad F_{B_{d}}=188(4) \mathrm{MeV},
\end{gathered}
$$

which contain smaller errors than quoted in [104].

We should also mention recent results from the Twisted Mass Collaboration 62

$$
\sqrt{\hat{B}_{B_{s}}} F_{B_{s}}=262(10) \mathrm{MeV}, \quad \sqrt{\hat{B}_{B_{d}}} F_{B_{d}}=216(10) \mathrm{MeV},
$$

which are not yet included in the FLAG average but having smaller errors are consistent with the latter

Evidently there is a big progress in determining all these relevant parameters but one would like to decrease the errors further and it appears that this should be possible in the coming years. Selected reviews about the status and prospects can be found in $62,121,125$.

\subsection{Step 3: $\Delta F=2$ Observables}

\subsubsection{Contributing operators}

In order to describe these processes in generality we begin by listing the operators which can contribute to $\Delta F=2$ observables in any extension of the SM. Specifying to the $K^{0}-\bar{K}^{0}$ system the full basis is given as follows 93,126 ;:

$$
\begin{aligned}
Q_{1}^{\mathrm{VLL}} & =\left(\bar{s} \gamma_{\mu} P_{L} d\right)\left(\bar{s} \gamma^{\mu} P_{L} d\right), \\
Q_{1}^{\mathrm{VRR}} & =\left(\bar{s} \gamma_{\mu} P_{R} d\right)\left(\bar{s} \gamma^{\mu} P_{R} d\right), \\
Q_{1}^{\mathrm{LR}} & =\left(\bar{s} \gamma_{\mu} P_{L} d\right)\left(\bar{s} \gamma^{\mu} P_{R} d\right), \\
Q_{2}^{\mathrm{LR}} & =\left(\bar{s} P_{L} d\right)\left(\bar{s} P_{R} d\right) \\
Q_{1}^{\mathrm{SLL}} & =\left(\bar{s} P_{L} d\right)\left(\bar{s} P_{L} d\right) \\
Q_{1}^{\mathrm{SRR}} & =\left(\bar{s} P_{R} d\right)\left(\bar{s} P_{R} d\right) \\
Q_{2}^{\mathrm{SLL}} & =\left(\bar{s} \sigma_{\mu \nu} P_{L} d\right)\left(\bar{s} \sigma^{\mu \nu} P_{L} d\right),
\end{aligned}
$$




\begin{tabular}{|c||c|c|c|c|}
\hline & $\left\langle Q_{1}^{\mathrm{LR}}\left(\mu_{H}\right)\right\rangle$ & $\left\langle Q_{2}^{\mathrm{LR}}\left(\mu_{H}\right)\right\rangle$ & $\left\langle Q_{1}^{\mathrm{SLL}}\left(\mu_{H}\right)\right\rangle$ & $\left\langle Q_{2}^{\mathrm{SLL}}\left(\mu_{H}\right)\right\rangle$ \\
\hline \hline$K^{0}-\bar{K}^{0}$ & -0.14 & 0.22 & -0.074 & -0.128 \\
\hline$B_{d^{0}}^{0} \bar{B}_{d}^{0}$ & -0.25 & 0.34 & -0.11 & -0.22 \\
\hline$B_{s}^{0}-\bar{B}_{s}^{0}$ & -0.37 & 0.51 & -0.17 & -0.33 \\
\hline
\end{tabular}

Table 2: Hadronic matrix elements $\left\langle Q_{i}^{a}\left(\mu_{H}\right)\right\rangle$ in units of $G e V^{3}$ at $\mu_{H}=1 \mathrm{TeV}$.

$$
Q_{2}^{\mathrm{SRR}}=\left(\bar{s} \sigma_{\mu \nu} P_{R} d\right)\left(\bar{s} \sigma^{\mu \nu} P_{R} d\right),
$$

where $P_{R, L}=\left(1 \pm \gamma_{5}\right) / 2$ and we suppressed colour indices as they are summed up in each factor. For instance $\bar{s} \gamma_{\mu} P_{L} d$ stands for $\bar{s}_{\alpha} \gamma_{\mu} P_{L} d_{\alpha}$ and similarly for other factors. For $B_{q}^{0}-\bar{B}_{q}^{0}$ mixing our conventions for operators are:

$$
\begin{aligned}
Q_{1}^{\mathrm{VLL}} & =\left(\bar{b} \gamma_{\mu} P_{L} q\right)\left(\bar{b} \gamma^{\mu} P_{L} q\right), \\
Q_{1}^{\mathrm{VRR}} & =\left(\bar{b} \gamma_{\mu} P_{R} q\right)\left(\bar{b} \gamma^{\mu} P_{R} q\right), \\
Q_{1}^{\mathrm{LR}} & =\left(\bar{b} \gamma_{\mu} P_{L} q\right)\left(\bar{b} \gamma^{\mu} P_{R} q\right), \\
Q_{2}^{\mathrm{LR}} & =\left(\bar{b} P_{L} q\right)\left(\bar{b} P_{R} q\right), \\
Q_{1}^{\mathrm{SLL}} & =\left(\bar{b} P_{L} q\right)\left(\bar{b} P_{L} q\right), \\
Q_{1}^{\mathrm{SRR}} & =\left(\bar{b} P_{R} q\right)\left(\bar{b} P_{R} q\right), \\
Q_{2}^{\mathrm{SLL}} & =\left(\bar{b} \sigma_{\mu \nu} P_{L} q\right)\left(\bar{b} \sigma^{\mu \nu} P_{L} q\right), \\
Q_{2}^{\mathrm{SRR}} & =\left(\bar{b} \sigma_{\mu \nu} P_{R} q\right)\left(\bar{b} \sigma^{\mu \nu} P_{R} q\right),
\end{aligned}
$$

As already mentioned in Step 2 the main theoretical uncertainties in $\Delta F=2$ transitions reside in the hadronic matrix elements of the contributing operators. These matrix elements are usually evaluated by lattice QCD at scales corresponding roughly to the scale of decaying hadron although in the case of $K$ meson decays, in order to improve the matching with the Wilson coefficients, the lattice calculations are performed these days at scales $\mu \approx 2 \mathrm{GeV}$. However, for the study of NP contributions it is useful, starting from their values at these low scales, to evaluate them at scales where NP is at work. This can be done by means of renormalization group methods and the corresponding analytic formulae to achieve this goal can be found in [126]. 


\begin{tabular}{|c||c|c|c|c|}
\hline & $\left\langle Q_{1}^{\mathrm{LR}}\left(m_{t}\right)\right\rangle$ & $\left\langle Q_{2}^{\mathrm{LR}}\left(m_{t}\right)\right\rangle$ & $\left\langle Q_{1}^{\mathrm{SLL}}\left(m_{t}\right)\right\rangle$ & $\left\langle Q_{2}^{\mathrm{SLL}}\left(m_{t}\right)\right\rangle$ \\
\hline \hline$K^{0}-\bar{K}^{0}$ & -0.11 & 0.18 & -0.064 & -0.107 \\
\hline$B_{d}^{0}-\bar{B}_{d}^{0}$ & -0.21 & 0.27 & -0.095 & -0.191 \\
\hline$B_{s}^{0}-\bar{B}_{s}^{0}$ & -0.30 & 0.40 & -0.14 & -0.29 \\
\hline
\end{tabular}

Table 3: Hadronic matrix elements $\left\langle Q_{i}^{a}\left(\mu_{t}\right)\right\rangle$ in units of $G e V^{3}$ at $m_{t}\left(m_{t}\right)$.

The most recent values of the matrix elements of the operators at a high scale $\mu_{H}=$ $1 \mathrm{TeV}$ are given in Table 2. The matrix elements of operators with $\mathrm{L}$ replaced by $\mathrm{R}$ are equal to the ones given in this table. The values in Table 2 correspond to the $\overline{\mathrm{MS}}$-NDR scheme and are based on lattice calculations in 127,128 for $K^{0}-\bar{K}^{0}$ system and in [129] for $B_{d, s}^{0}-\bar{B}_{d . s}^{0}$ systems. For the $K^{0}-\bar{K}^{0}$ system we have just used the average of the results in [127, 128 that are consistent with each other ${ }^{3}$. As the values of the relevant $B_{i}$ parameters in these papers have been evaluated at $\mu=3 \mathrm{GeV}$ and $4.2 \mathrm{GeV}$, respectively, we have used the formulae in [126] to obtain the values of the matrix elements in question at $\mu_{H}$. For simplicity we choose this scale to be $M_{H}$ but any scale of this order would give the same results for the physical quantities up to NNLO QCD corrections that are negligible at these high scales. The renormalization scheme dependence of the matrix elements is canceled by the one of the Wilson coefficients as discussed below.

In the case of tree-level SM $Z$ and SM Higgs exchanges we evaluate the matrix elements at $m_{t}\left(m_{t}\right)$ as the inclusion of NLO QCD corrections allows us to choose any scale of $\mathcal{O}\left(M_{H}\right)$ without changing physical results. The values of hadronic matrix elements at $m_{t}\left(m_{t}\right)$ in the $\overline{\mathrm{MS}}-\mathrm{NDR}$ scheme are given in Table 3 .

The Wilson coefficients of these operators depend on the short distance properties of a given theory. They can be directly expressed in terms of the couplings $\Delta_{L, R}^{i j}\left(Z^{\prime}\right)$ and $\Delta_{L, R}^{i j}\left(H^{0}\right)$ in the case of tree-level gauge boson and scalar exchanges. In models with GIM mechanism at work they are given in terms of loop functions. Then couplings $\Delta_{L, R}^{i j}\left(W^{\prime+}\right)$ and $\Delta_{L, R}^{i j}\left(H^{+}\right)$enter the game.

\subsubsection{Standard Model Results}

In the SM only the operator $Q_{1}^{\mathrm{VLL}}$ contributes to each meson system. With the information gained in Steps 1 and 2 at hand we are ready to calculate the SM values for the

\footnotetext{
${ }^{3}$ The recent results using staggered fermions from SWME collaboration in $K^{0}-\bar{K}^{0}$ system 130 are not included here. While for $Q_{1,2}^{\mathrm{SLL}}$ this group obtains results consistent with 127, 128, the matrix elements of $Q_{1,2}^{\mathrm{LR}}$ are by $50 \%$ larger. Let us hope this difference will be clarified soon.
} 
five super stars in (35). To this end we recall the formulae for $\Delta M_{d, s}, S_{\psi K_{S}}, S_{\psi \phi}$, and $\varepsilon_{K}$.

Defining

$$
\lambda_{i}^{(K)}=V_{i s}^{*} V_{i d}, \quad \lambda_{t}^{(d)}=V_{t b}^{*} V_{t d}, \quad \lambda_{t}^{(s)}=V_{t b}^{*} V_{t s},
$$

we have first

$$
\begin{aligned}
& \Delta M_{s}=\frac{G_{F}^{2}}{6 \pi^{2}} M_{W}^{2} m_{B_{s}}\left|\lambda_{t}^{(s)}\right|^{2} F_{B_{s}}^{2} \hat{B}_{B_{s}} \eta_{B} S_{0}\left(x_{t}\right), \\
& \Delta M_{d}=\frac{G_{F}^{2}}{6 \pi^{2}} M_{W}^{2} m_{B_{d}}\left|\lambda_{t}^{(d)}\right|^{2} F_{B_{d}}^{2} \hat{B}_{B_{d}} \eta_{B} S_{0}\left(x_{t}\right) .
\end{aligned}
$$

which result from $(q=d, s)$

$$
\left(M_{12}^{q}\right)_{\mathrm{SM}}^{*}=\frac{G_{F}^{2}}{12 \pi^{2}} F_{B_{q}}^{2} \hat{B}_{B_{q}} m_{B_{q}} M_{W}^{2}\left[\left(\lambda_{t}^{(q)}\right)^{2} \eta_{B} S_{0}\left(x_{t}\right)\right]
$$

and

$$
\Delta M_{q}=2\left|M_{12}^{q}\right| .
$$

Here $x_{t}=m_{t}^{2} / M_{W}^{2}, \eta_{B}=0.55$ is a QCD factor and

$$
S_{0}\left(x_{t}\right)=\frac{4 x_{t}-11 x_{t}^{2}+x_{t}^{3}}{4\left(1-x_{t}\right)^{2}}-\frac{3 x_{t}^{2} \log x_{t}}{2\left(1-x_{t}\right)^{3}}=2.31\left[\frac{\bar{m}_{\mathrm{t}}\left(m_{\mathrm{t}}\right)}{163 \mathrm{GeV}}\right]^{1.52} .
$$

We find then three useful formulae $\left(\left|V_{t b}\right|=1\right)$

$$
\begin{gathered}
\Delta M_{s}=17.7 / \mathrm{ps} \cdot\left[\frac{\sqrt{\hat{B}_{B_{s}}} F_{B_{s}}}{267 \mathrm{MeV}}\right]^{2}\left[\frac{S_{0}\left(x_{t}\right)}{2.31}\right]\left[\frac{\left|V_{t s}\right|}{0.0402}\right]^{2}\left[\frac{\eta_{B}}{0.55}\right], \\
\Delta M_{d}=0.51 / \mathrm{ps} \cdot\left[\frac{\sqrt{\hat{B}_{B_{d}}} F_{B_{d}}}{218 \mathrm{MeV}}\right]^{2}\left[\frac{S_{0}\left(x_{t}\right)}{2.31}\right]\left[\frac{\left|V_{t d}\right|}{8.5 \cdot 10^{-3}}\right]^{2}\left[\frac{\eta_{B}}{0.55}\right]
\end{gathered}
$$

and

$$
R_{\Delta M_{B}}=\frac{\Delta M_{d}}{\Delta M_{s}}=\frac{m_{B_{d}}}{m_{B_{s}}} \frac{\hat{B}_{d}}{\hat{B}_{s}} \frac{F_{B_{d}}^{2}}{F_{B_{s}}^{2}}\left|\frac{V_{t d}}{V_{t s}}\right|^{2} \equiv \frac{m_{B_{d}}}{m_{B_{s}}} \frac{1}{\xi^{2}}\left|\frac{V_{t d}}{V_{t s}}\right|^{2} .
$$

The mixing induced CP-asymmetries are given within the SM simply by

$$
S_{\psi K_{S}}=\sin (2 \beta), \quad S_{\psi \phi}=\sin \left(2\left|\beta_{s}\right|\right) .
$$

They are the coefficients of $\sin \left(\Delta M_{d} t\right)$ and $\sin \left(\Delta M_{s} t\right)$ in the time dependent asymmetries in $B_{d}^{0} \rightarrow \psi K_{S}$ and $B_{s}^{0} \rightarrow \psi \phi$, respectively. 


\begin{tabular}{|c||c|c|c|c|c|c|}
\hline$\left|V_{u b}\right| \times 10^{3}$ & 3.1 & 3.4 & 3.7 & 4.0 & 4.3 & Experiment \\
\hline \hline$\left|\varepsilon_{K}\right| \times 10^{3}$ & 1.76 & 1.91 & 2.05 & 2.19 & 2.33 & $2.228(11)$ \\
$\mathcal{B}\left(B^{+} \rightarrow \tau^{+} \nu_{\tau}\right) \times 10^{4}$ & 0.58 & 0.70 & 0.83 & 0.97 & 1.12 & $1.14(22)$ \\
$(\sin 2 \beta)_{\text {true }}$ & 0.619 & 0.671 & 0.720 & 0.766 & 0.808 & $0.679(20)$ \\
$S_{\psi \phi}$ & 0.032 & 0.035 & 0.038 & 0.042 & 0.046 & $0.001(9)$ \\
$\Delta M_{s}\left[\mathrm{ps}^{-1}\right](\mathrm{I})$ & 17.5 & 17.5 & 17.5 & 17.6 & 17.6 & $17.69(8)$ \\
$\Delta M_{d}\left[\mathrm{ps}^{-1}\right](\mathrm{I})$ & 0.52 & 0.51 & 0.51 & 0.52 & 0.52 & $0.507(4)$ \\
$\Delta M_{s}\left[\mathrm{ps}^{-1}\right](\mathrm{II})$ & 19.2 & 19.2 & 19.2 & 19.3 & 19.3 & $17.72(4)$ \\
$\Delta M_{d}\left[\mathrm{ps}^{-1}\right](\mathrm{II})$ & 0.56 & 0.56 & 0.56 & 0.57 & 0.57 & $0.510(4)$ \\
$\left|V_{t d}\right| \times 10^{3}$ & 8.56 & 8.54 & 8.54 & 8.56 & 8.57 & -- \\
$\left|V_{t s}\right| \times 10^{3}$ & 40.0 & 40.0 & 40.0 & 40.0 & 40.0 & -- \\
\hline
\end{tabular}

Table 4: SM prediction for various observables as functions of $\left|V_{u b}\right|$ and $\gamma=68^{\circ}$. The two results for $\Delta M_{s, d}$ correspond to two sets of the values of $F_{B_{s}} \sqrt{\hat{B}_{B_{s}}}$ and $F_{B_{d}} \sqrt{\hat{B}_{B_{d}}}$ : central values in Table 1 (I) and older values in (36) (II).

For the CP-violating parameter $\varepsilon_{K}$ we have

$$
\varepsilon_{K}=\frac{\kappa_{\varepsilon} e^{i \varphi_{\varepsilon}}}{\sqrt{2}\left(\Delta M_{K}\right)_{\exp }}\left[\Im\left(M_{12}^{K}\right)_{\mathrm{SM}}\right]
$$

where $\varphi_{\varepsilon}=(43.51 \pm 0.05)^{\circ}$ and $\kappa_{\varepsilon}=0.94 \pm 0.02\left[34\right.$ 113] takes into account that $\varphi_{\varepsilon} \neq \frac{\pi}{4}$ and includes long distance effects in $\Im\left(\Gamma_{12}\right)$ and $\Im\left(M_{12}\right)$. Moreover

$$
\left(M_{12}^{K}\right)_{\mathrm{SM}}^{*}=\frac{G_{F}^{2}}{12 \pi^{2}} F_{K}^{2} \hat{B}_{K} m_{K} M_{W}^{2}\left[\lambda_{c}^{2} \eta_{c c} S_{0}\left(x_{c}\right)+\lambda_{t}^{2} \eta_{t t} S_{0}\left(x_{t}\right)+2 \lambda_{c} \lambda_{t} \eta_{c t} S_{0}\left(x_{c}, x_{t}\right)\right],
$$

where $\eta_{i}$ are QCD factors given in Table 1 and $S_{0}\left(x_{c}, x_{t}\right)$ can be found in [131].

In Table 4 we summarize the results for $\left|\varepsilon_{K}\right|, \mathcal{B}\left(B^{+} \rightarrow \tau^{+} \nu_{\tau}\right), \Delta M_{s, d},(\sin 2 \beta)_{\text {true }}$, $\Delta M_{s, d},\left|V_{t d}\right|$ and $\left|V_{t s}\right|$ obtained from (32), setting

$$
\left|V_{u s}\right|=0.2252, \quad\left|V_{c b}\right|=0.0409, \quad \gamma=68^{\circ},
$$

and choosing five values for $\left|V_{u b}\right|$. Two of them correspond to two scenarios defined in Section 2. The value of $\gamma$ is close to its most recent value from $B \rightarrow D K$ decays obtained by LHCb using $3 \mathrm{fb}^{-1}$ and neglecting $D^{0}-\bar{D}^{0}$ mixing [132]

$$
\gamma=(67.2 \pm 12)^{\circ}, \quad(\mathrm{LHCb})
$$

and to the extraction from U-spin analysis of $B_{s} \rightarrow K^{+} K^{-}$and $B_{d} \rightarrow \pi^{+} \pi^{-}$decays $\left(\gamma=(68.2 \pm 7.1)^{\circ}\right)[133$. In [134 both $B \rightarrow D K$ and $B \rightarrow D \pi$ decays are used and 
furthermore $D^{0}-\bar{D}^{0}$ mixing fully included and the combination of results gives as best-fit value $\gamma=72.6^{\circ}$ and the confidence interval $\gamma \in[55.4,82.3]^{\circ}$ at $68 \% \mathrm{CL}$. We do not show the uncertainties in SM predictions but just quote rough estimate of them:

$\left|\varepsilon_{K}\right|: \pm 11 \%, \quad \mathcal{B}\left(B^{+} \rightarrow \tau^{+} \nu_{\tau}\right): \pm 15 \%, \quad \Delta M_{s, d}: \pm 10 \%, \quad S_{\psi K_{S}}: \pm 3.0 \%$.

In order to show the importance of precise values of the non-perturbative parameters we show the results for present central values of $F_{B_{s}} \sqrt{\hat{B}_{B_{s}}}$ and $F_{B_{d}} \sqrt{\hat{B}_{B_{d}}}$ in Table 1 (I) and for the older values in (36) indicated by (II).

We observe that while $\Delta M_{s, d},\left|V_{t d}\right|$ and $\left|V_{t s}\right|$, practically do not depend on $\left|V_{u b}\right|$, this is not the case for the remaining observables, although the $\left|V_{u b}\right|$ dependence in $S_{\psi \phi}$ is very weak. Clearly the data show that it is difficult to fit simultaneously $\varepsilon_{K}$ and $S_{\psi \phi}$ within the SM but the character of the NP which could cure these tensions depends on the choice of $\left|V_{u b}\right|$. On the other hand the agreement of the SM with the data on $\Delta M_{s}$ and $\Delta M_{d}$ is very good. In particular for the set (I) we find

$$
\left(\frac{\Delta M_{s}}{\Delta M_{d}}\right)_{\mathrm{SM}}=34.1 \pm 3.0 \quad \exp : \quad 34.7 \pm 0.3
$$

in excellent agreement with the data.

We learn the following lessons to be remembered when we start investigating models beyond the SM:

\section{Lesson 1:}

We learn that in the case of exclusive determination of $\left|V_{u b}\right|$ any NP model that pretends to be able to remove or soften the observed departures from the data should simultaneously:

- Enhance $\left|\varepsilon_{K}\right|$ by roughly $20 \%$ without affecting significantly the result for $S_{\psi K_{S}}$.

- Suppress slightly $\Delta M_{s}$ and $\Delta M_{d}$ without affecting significantly their ratio in the case of the set (II). This suppression is not required if the set (I) is used.

\section{Lesson 2:}

We learn that in the case of inclusive determination of $\left|V_{u b}\right|$ any NP model that pretends to be able to remove or soften the observed departures from the data should simultaneously:

- Suppress $S_{\psi K_{S}}$ by roughly $20 \%$ without affecting significantly the result for $\left|\varepsilon_{K}\right|$

- Suppress slightly $\Delta M_{s}$ and $\Delta M_{d}$ without affecting significantly their ratio in the case of the set (II). This suppression is not required if the set (I) is used. 
Clearly $\left|V_{u b}\right|$ could have an intermediate value but we find that a more transparent picture emerges for these two values.

\section{Lesson 3:}

The next lesson comes from HQAG [112]:

$$
S_{\psi \phi}=-\left(0.04_{-0.13}^{+0.10}\right), \quad S_{\psi \phi}^{\mathrm{SM}}=0.038 \pm 0.005,
$$

where we have shown also SM prediction and the experimental error on $S_{\psi \phi}$ has been obtained by adding statistical and systematic errors in quadrature. Indeed it looks like the SM still survived another test: mixing induced CP-violation in $B_{s}$ decays is significantly lower than in $B_{d}$ decays as expected in the SM already for 25 years. However from the present perspective $S_{\psi \phi}$ could still be found in the range

$$
-0.20 \leq S_{\psi \phi} \leq 0.20
$$

and finding it to be negative would be a clear signal of NP. Moreover finding it above 0.1 would also be a signal of NP but not as pronounced as the negative value. The question then arises whether this NP is somehow correlated with the one related to the anomalies identified above. We will return to this issue in the course of our presentation.

\section{Lesson 4:}

The final lesson comes from the recent analysis in $\left[47 \mid\right.$ were the values $\left|V_{c b}\right|=(42.4(9)) \times$ $10^{-3}[116]$ and $\left|V_{u b}\right|=(3.6 \pm 0.3) \times 10^{-3}[103]$ have been used. For such values there is an acceptable simultaneous agreement of the SM with both $S_{\psi K_{S}}$ and $\varepsilon_{K}$ but then

$$
\Delta M_{s}=18.8 \mathrm{ps}^{-1}, \quad \Delta M_{d}=0.530 \mathrm{ps}^{-1},
$$

slightly above the data.

This discussion shows how important is the determinations of the CKM and and nonperturbative parameters if we want to identify NP indirectly through flavour violating processes. We will return to this point below and refer to [46, 47], where extensive numerical analysis of this issue has been presented in the context of models with treelevel FCNC transitions.

\subsubsection{Going Beyond the Standard Model}

In view of NP contributions, required to remove the anomalies just discussed, we have to generalize the formulae of the SM. First for $M_{12}^{K}, M_{12}^{d}$ and $M_{12}^{s}$, that govern the analysis of $\Delta F=2$ transitions in any extension of the SM we have

$$
M_{12}^{i}=\left(M_{12}^{i}\right)_{\mathrm{SM}}+\left(M_{12}^{i}\right)_{\mathrm{NP}}, \quad(i=K, d, s),
$$

with $\left(M_{12}^{i}\right)_{\mathrm{SM}}$ given in 46 and $(54)$. 
For the mass differences in the $B_{d, s}^{0}-\bar{B}_{d, s}^{0}$ systems we have then

$$
\Delta M_{q}=2\left|\left(M_{12}^{q}\right)_{\mathrm{SM}}+\left(M_{12}^{q}\right)_{\mathrm{NP}}\right| \quad(q=d, s) .
$$

Now

$$
M_{12}^{q}=\left(M_{12}^{q}\right)_{\mathrm{SM}}+\left(M_{12}^{q}\right)_{\mathrm{NP}}=\left(M_{12}^{q}\right)_{\mathrm{SM}} C_{B_{q}} e^{2 i \varphi_{B_{q}}},
$$

where

$$
\left(M_{12}^{d}\right)_{\mathrm{SM}}=\left|\left(M_{12}^{d}\right)_{\mathrm{SM}}\right| e^{2 i \beta}, \quad\left(M_{12}^{s}\right)_{\mathrm{SM}}=\left|\left(M_{12}^{s}\right)_{\mathrm{SM}}\right| e^{2 i \beta_{s}} .
$$

The phases $\beta$ and $\beta_{s}$ are defined in (28) and one has approximately $\beta \approx(22 \pm 3)^{\circ}$ and $\beta_{s} \simeq-1^{\circ}$ with precise values depending on $\left|V_{u b}\right|$. We find then

$$
\Delta M_{q}=\left(\Delta M_{q}\right)_{\mathrm{SM}} C_{B_{q}},
$$

and

$$
S_{\psi K_{S}}=\sin \left(2 \beta+2 \varphi_{B_{d}}\right), \quad S_{\psi \phi}=\sin \left(2\left|\beta_{s}\right|-2 \varphi_{B_{s}}\right) .
$$

Thus in the presence of non-vanishing $\varphi_{B_{d}}$ and $\varphi_{B_{s}}$ these two asymmetries do not measure $\beta$ and $\beta_{s}$ but $\left(\beta+\varphi_{B_{d}}\right)$ and $\left(\left|\beta_{s}\right|-\varphi_{B_{s}}\right)$, respectively.

It should be remarked that the experimental results are usually given for the phase

$$
\phi_{s}=2 \beta_{s}+\phi^{\mathrm{NP}}
$$

so that

$$
S_{\psi \phi}=-\sin \left(\phi_{s}\right), \quad 2 \varphi_{B_{s}}=\phi^{\mathrm{NP}} .
$$

In particular the minus sign in this equation should be remembered when comparing our results with those quoted by the LHCb.

Next, the parameter $\varepsilon_{K}$ is given by

$$
\varepsilon_{K}=\frac{\kappa_{\varepsilon} e^{i \varphi_{\varepsilon}}}{\sqrt{2}\left(\Delta M_{K}\right)_{\exp }}\left[\Im\left(M_{12}^{K}\right)_{\mathrm{SM}}+\Im\left(M_{12}^{K}\right)_{\mathrm{NP}}\right] .
$$

Finally, the ratio in (51) can be modified

$$
R_{\Delta M_{B}}=\frac{\Delta M_{d}}{\Delta M_{s}}=\frac{m_{B_{d}}}{m_{B_{s}}} \frac{1}{\xi^{2}}\left|\frac{V_{t d}}{V_{t s}}\right|^{2} r(\Delta M),
$$

where the departure of $r(\Delta M)$ from unity signals non-MFV sources at work. In this review we only rarely consider $\Delta M_{K}$ as it is subject to large hadronic uncertainties. Moreover generally $\varepsilon_{K}$ gives a stronger constraint on NP.

We will now investigate which of the models introduced in Section 3 could remove the anomalies just discussed dependently whether exclusive or inclusive value of $\left|V_{u b}\right|$ has been chosen by nature and which models are put under significant pressure in both cases. In the latter case the hope is that the final value for $\left|V_{u b}\right|$ will be some average 
of inclusive and exclusive determinations, that is in the ballpark of $\left|V_{u b}\right|=3.7 \times 10^{-3}$. If this will turn out not to be the case the latter models are then either close to being ruled out or are incomplete requiring new sources of flavour and/or CP violation in order to agree with the data. As we will soon see the simplest models considered by us have a sufficiently low number of parameters that concrete answers about their ability to remove the anomalies in question can be given, in particular when subsequent steps will be considered.

\subsubsection{Constrained Minimal Flavour Violation (CMFV)}

The flavour structure in this class of models implies that the mixing induced CP asymmetries $S_{\psi K_{S}}$ and $S_{\psi \phi}$ are not modified with respect to the SM and the expressions in (52) still apply.

This structure also implies the flavour universality of loop functions contributing to various processes that is broken only by the CKM factors multiplying these functions. In the case of $\Delta F=2$ processes considered here this means that in this class of models $\mathrm{NP}$ can only modify the loop function $S_{0}\left(x_{t}\right)$ to some real valued function $S(v)$ without modifying the values of the CKM parameters that have been determined in Step 1 without any influence of NP.

Now, it has been demonstrated diagrammatically in 60 that in the context of CMFV:

$$
S_{0}\left(x_{t}\right) \leq S(v) .
$$

This simply implies that $\left|\varepsilon_{K}\right|, \Delta M_{d}$ and $\Delta M_{s}$ can only be enhanced in this class of models. Moreover, this happens in a correlated manner. A correlation between $\left|\varepsilon_{K}\right|$, $\Delta M_{d}$ and $S_{\psi K_{S}}$ within the SM has been pointed out in [58,59] and generalized to all models with CMFV in [55]. This correlation follows from the universality of $S(v)$ and the fact that in all CMFV models considered, only the term in $\varepsilon_{K}$ involving $\left(V_{t s}^{*} V_{t d}\right)^{2}$ is affected visibly by NP with the remaining terms described by the SM.

Here we want to look at this correlation from a bit different point of view. In fact eliminating the one-loop function $S(v)$ in $\varepsilon_{K}$ in favour of $\Delta M_{d}$ and using also $\Delta M_{s}$ one can find universal expressions for $S_{\psi K_{S}}$ and the angle $\gamma$ in the UUT that depend only on $\left|V_{u s}\right|,\left|V_{c b}\right|$, known from tree-level decays, and non-perturbative parameters entering the evaluation of $\varepsilon_{K}$ and $\Delta M_{s, d}$. They are valid for all CMFV models. Therefore, once the data on $\left|V_{u s}\right|,\left|V_{c b}\right|, \varepsilon_{K}$ and $\Delta M_{s, d}$ are taken into account one is able in this framework to predict not only $S_{\psi K_{S}}$ and $\gamma$ but also $\left|V_{u b}\right|$.

Explicitly we find first

$$
S_{\psi K_{S}}=\sin 2 \beta=\frac{1}{b \Delta M_{d}}\left[\frac{\left|\varepsilon_{K}\right|}{\left|V_{c b}\right|^{2} \hat{B}_{K}}-a\right],
$$


where

$$
a=r_{\varepsilon} R_{t} \sin \beta\left[\eta_{c t} S_{0}\left(x_{t}, x_{c}\right)-\eta_{c c} x_{c}\right], \quad b=\frac{\eta_{t t}}{\eta_{B}} \frac{r_{\varepsilon}}{2 r_{d}\left|V_{u s}\right|^{2}} \frac{1}{F_{B_{d}}^{2} \hat{B}_{B_{d}}}
$$

with

$$
r_{\varepsilon}=\kappa_{\varepsilon}\left|V_{u s}\right|^{2} \frac{G_{F}^{2} F_{K}^{2} m_{K} M_{W}^{2}}{6 \sqrt{2} \pi^{2} \Delta M_{K}}, \quad r_{d}=\frac{G_{F}^{2}}{6 \pi^{2}} M_{W}^{2} m_{B_{d}}
$$

The following remarks should be made

- The second term $a$ in the parenthesis in 73 is roughly by a factor of 4-5 smaller than the first term. It depends on $\beta$ through $\sin \beta$ and $\left(\lambda=\left|V_{u s}\right|\right)$

$$
R_{t}=\eta_{R} \frac{\xi}{\left|V_{u s}\right|} \sqrt{\frac{\Delta M_{d}}{\Delta M_{s}}} \sqrt{\frac{m_{B_{s}}}{m_{B_{d}}}}, \quad \eta_{R}=1-\left|V_{u s}\right| \xi \sqrt{\frac{\Delta M_{d}}{\Delta M_{s}}} \sqrt{\frac{m_{B_{s}}}{m_{B_{d}}}} \cos \beta+\frac{\lambda^{2}}{2}+\mathcal{O}\left(\lambda^{4}\right),
$$

but this dependence is very weak and $0.34 \leq a \leq 0.41$ in the full range of parameters considered.

- The ratio of $\eta_{t t} / \eta_{B}$ is independent of NP.

- With $R_{t}$ and $\beta$ determined in this manner one can calculate $\gamma$ and $\left|V_{u b}\right|$ by means of 29 and 33 .

- The element $\left|V_{c b}\right|$ appears only as square in these expressions and not as $\left|V_{c b}\right|^{4}$ in $\varepsilon_{K}$, which improves the accuracy of the determination.

We should emphasize that in this determination the experimental input $\Delta M_{s, d}$ and $\varepsilon_{K}$ is very precise. $\left|V_{u s}\right|$ is known very well and $\left|V_{c b}\right|$ is better known than $\left|V_{u b}\right|$ from tree-level decays.

Setting then the experimental values of $\Delta M_{s, d}, \varepsilon_{K}$ and $\left|V_{c b}\right|$ as well as central values of the non-perturbative parameters in Table 1 into (73) we find

$$
S_{\psi K_{S}}=0.81(0.87) \Rightarrow \beta=27\left(30^{\circ}\right), \quad R_{t}=0.92(0.92)
$$

and thus

$$
R_{b}=0.46(0.50), \quad\left|V_{u b}\right|=0.0043(0.0047), \quad \gamma=67.2\left(66.4^{\circ}\right),
$$

where the values in parentheses correspond to the input in (36). This demonstrates sensitivity to the non-perturbative parameters.

While a sophisticated analysis including all uncertainties would somewhat wash out these results, the message from this exercise is clear. The fact that $S_{\psi K_{S}}$ is much larger than the data requires the presence of new CP-violating phases, although with the most recent lattice input these phases can be smaller. This exercise is equivalent to the one 


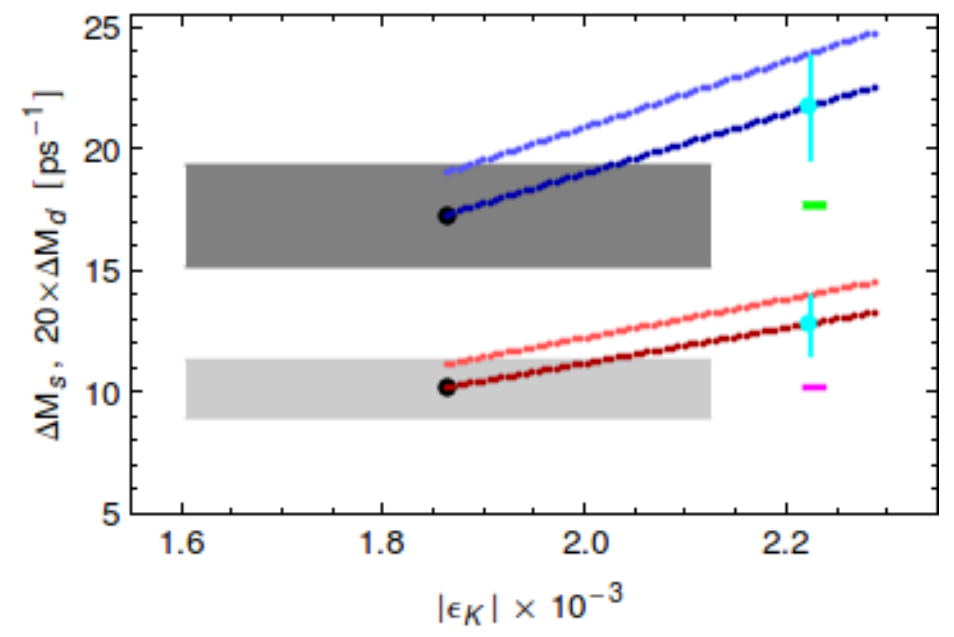

Figure 5: $\Delta M_{s}$ (blue) and 20. $\Delta M_{d}$ (red) as functions of $\left|\varepsilon_{K}\right|$ in models with $C M F V$ for Scenario 1 chosen by these models [20]. The short green and magenta lines represent the data, while the large black and grey regions the SM predictions. For the light blue and light red line the old values from (36) are used and for dark blue and dark red the new ones from Table 1. More information can be found in the text.

performed in [33], where $\varepsilon_{K}$ has been set to its experimental value but $\sin 2 \beta$ was free. On the other hand setting $S_{\psi K_{S}}$ to its experimental value but keeping $\varepsilon_{K}$ free as done in [34] one finds that $\left|\varepsilon_{K}\right|$ is significantly below the data. Yet, this difficulty can be resolved in CMFV models by increasing the value of $S(v)$. While, the latter approach is clearly legitimate, it hides possible problems of CMFV as it assumes that this NP scenario can describe the data on $\Delta M_{s, d}$ and $\varepsilon_{K}$ simultaneously, which as we will now show is not really the case.

Indeed, with respect to the anomalies discussed above we note that

- CMFV models favour the exclusive determination of $\left|V_{u b}\right|$ as only then they are capable of reproducing the experimental value of $S_{\psi K_{S}}$.

- $\left|\varepsilon_{K}\right|$ can be naturally enhanced by increasing the value of $S(v)$ thereby solving the $\left|\varepsilon_{K}\right|-S_{\psi K_{S}}$ tension.

- $\Delta M_{s, d}$ are enhanced simultaneously with the ratio $\Delta M_{s} / \Delta M_{d}$ unchanged with respect to the SM $(r(\Delta M)=1)$. While the latter property is certainly good news, the enhancements of $\Delta M_{s}$ and $\Delta M_{d}$ are clearly problematic. Therefore the present values of hadronic matrix elements imply new tensions, namely the $\left|\varepsilon_{K}\right|-\Delta M_{s, d}$ tensions pointed out in 20,135 .

In Fig. 5 we plot $\Delta M_{s}$ and $\Delta M_{d}$ as functions of $\left|\varepsilon_{K}\right|$. In obtaining this plot we have simply varied the master one-loop $\Delta F=2$ function $S$ keeping CKM parameters and 
other input parameters fixed. The value of $S$ at which central experimental value of $\left|\varepsilon_{K}\right|$ is reproduced turns out to be $S=2.9$ to be compared with $S_{\mathrm{SM}}=2.31$. At this value the central values of $\Delta M_{s, d}$ read

$$
\Delta M_{d}=0.64(6) \mathrm{ps}^{-1} \quad\left(0.69(6) \mathrm{ps}^{-1}\right), \quad \Delta M_{s}=21.7(2.1) \mathrm{ps}^{-1} \quad\left(23.9(2.1) \mathrm{ps}^{-1}\right) .
$$

They both differ from experimental values by $3 \sigma$. The error on $\left|\varepsilon_{K}\right|$ coming dominantly from the error of $\left|V_{c b}\right|$ and the error of the QCD factor $\eta_{c c}$ in the charm contribution 114 is however disturbing. Clearly this plot gives only some indication for possible difficulties of the CMFV models and we need a significant decrease of theoretical errors in order to see how solid this result is.

In summary, we observe that simultaneous good agreement for $\varepsilon_{K}$ and $\Delta M_{s, d}$ with the data is difficult to achieve in this NP scenario. It also implies that to improve the agreement with data we need at least one of the following four ingredients:

- Modification of the values of

$$
\left|V_{c b}\right|, \quad F_{B_{s}} \sqrt{\hat{B}_{B_{s}}}, \quad F_{B_{d}} \sqrt{\hat{B}_{B_{d}}}
$$

- New CP phases, flavour violating and/or flavour blind,

- New flavour violating contributions beyond the CKM matrix,

- New local operators which could originate in tree-level heavy gauge boson or scalar exchanges. They could also be generated at one-loop level.

The first possibility has been addressed in [45], where the experimental values of $\Delta M_{s, d}$, $\varepsilon_{K},\left|V_{u s}\right|$ and $S_{\psi K_{S}}$ have been used as input and $\hat{B}_{K}$ has been set to 0.75 in perfect agreement with the lattice results and the large $N$ approach $[136 \sqrt{139}$. Subsequently the parameters in 80 have been calculated as functions of $S(v)$ and $\gamma$ in order to see whether there is any hope for removing all the tensions in CMFV simultaneously in case the future more precise determinations of $F_{B_{s}} \sqrt{\hat{B}_{B_{s}}}, F_{B_{d}} \sqrt{\hat{B}_{B_{d}}}$ and $\left|V_{c b}\right|$ would result in different values than the present ones. The results of [45] can be summarized briefly as follows:

- The tension between $\varepsilon_{K}$ and $\Delta M_{s, d}$ in CMFV models accompanied with $\left|\varepsilon_{K}\right|$ being smaller than the data within the SM, cannot be removed by varying $S(v)$ when the present input parameters in Table 1 are used.

- Rather the value of $\left|V_{c b}\right|$ has to be increased and the values of $F_{B_{s}} \sqrt{\hat{B}_{B_{s}}}$ and $F_{B_{d}} \sqrt{\hat{B}_{B_{d}}}$ decreased relatively to the presently quoted lattice values. These enhancements and suppressions are correlated with each other and depend on $\gamma$. Setting the QCD corrections $\eta_{i j}$ at their central values one finds the results in Table 5 . 
- However, the present significant uncertainty in $\eta_{c c}$ softens these problems. Yet, it turns out that the knowledge of long distance contributions to $\Delta M_{K}$ accompanied by the very precise experimental value of the latter allows a significant reduction of the present uncertainty in the value of the QCD factor $\eta_{c c}$ under the plausible assumption that $\Delta M_{K}$ in CMFV models is fully dominated by the SM contribution. Indeed, using the large $N$ estimate of long distance contribution to $\Delta M_{K}[139$ we find

$$
\eta_{c c} \approx 1.70 \pm 0.21
$$

which implies the reduction of the theoretical error in $\varepsilon_{K}$ and in turn the reduction of the error in the extraction of the favoured value of $\left|V_{c b}\right|$ in the CMFV framework.

We should remark that the reduction of the error in $\eta_{c c}$ by a factor of more than 3.5 relatively to the one resulting from direct calculation [114 is significant as the uncertainty in $\varepsilon_{K}$ from $\eta_{c c}$ alone is reduced from roughly $7 \%$ to $2 \%$ and is consequently lower than the present uncertainty of $3 \%$ from $\eta_{c t}$. Yet, this reduction cannot be appreciated at present as by far the dominant uncertainty in $\varepsilon_{K}$ comes from $\left|V_{c b}\right|$.

In Fig. 6 on the left hand side we show the correlation between $F_{B_{d}} \sqrt{\hat{B}_{B_{d}}}$ and $\left|V_{c b}\right|$ for $\gamma \in\left[63^{\circ}, 71^{\circ}\right]$. Analogous correlation between $F_{B_{s}} \sqrt{\hat{B}_{B_{s}}}$ and $\left|V_{c b}\right|$ is shown on the right hand side. The dark gray boxes represent the present values of the parameters as given in Table 1, while the light gray the ones from (36). The vertical dark gray lines show where the dark gray boxes end, respectively. In these plots we show the anatomy of various uncertainties with different ranges described in the figure caption. We observe that the reduced error on $\eta_{c c}$ corresponding to the cyan region decreased the allowed region which with future lattice calculations could be decreased further. Comparing the blue and cyan regions we note that the reduction in the error on $\eta_{c t}$ would be welcomed as well. It should also be stressed that in a given CMFV model with fixed $S(v)$ the uncertainties are reduced further. This is illustrated with the black range for the case of the SM. Finally an impact on Fig. 6 will have a precise measurement of $\gamma$ or equivalently precise lattice determination of $\xi$. We illustrate this impact in Fig. 7 by setting in the plots of Fig. $6 \gamma=(67 \pm 1)^{\circ}$. Further details can be found in [45].

We note that the most recent values of $F_{B_{s}} \sqrt{\hat{B}_{B_{s}}}$ and $F_{B_{d}} \sqrt{\hat{B}_{B_{d}}}$ softened significantly the problems of CMFV in question, even if still an enhanced value of $\left|V_{c b}\right|$ is required. For instance, in accordance with the lesson 4 above, if one would ignore the present exclusive determination of $\left|V_{c b}\right|$ and used the most recent inclusive determination [116

$$
\left|V_{c b}\right|=(42.42 \pm 0.86) \times 10^{-3}
$$

CMFV would be in a much better shape but also the SM-like values for $S(v)$ would be favoured. We are looking forward to the improved lattice calculations and improved determinations of $\left|V_{c b}\right|$ in order to see whether CMFV will survive flavour precision tests. 


\begin{tabular}{|c|c||c|c|c|c|c|c|c|c|}
\hline$S(v)$ & $\gamma$ & $\left|V_{c b}\right|$ & $\left|V_{u b}\right|$ & $\left|V_{t d}\right|$ & $\left|V_{t s}\right|$ & $F_{B_{s}} \sqrt{\hat{B}_{B_{s}}}$ & $F_{B_{d}} \sqrt{\hat{B}_{B_{d}}}$ & $\xi$ & $\mathcal{B}\left(B^{+} \rightarrow \tau^{+} \nu\right)$ \\
\hline \hline 2.31 & $63^{\circ}$ & 43.6 & 3.69 & 8.79 & 42.8 & 252.7 & 210.0 & 1.204 & 0.822 \\
2.5 & $63^{\circ}$ & 42.8 & 3.63 & 8.64 & 42.1 & 247.1 & 205.3 & 1.204 & 0.794 \\
2.7 & $63^{\circ}$ & 42.1 & 3.56 & 8.49 & 41.4 & 241.8 & 200.9 & 1.204 & 0.768 \\
\hline 2.31 & $67^{\circ}$ & 42.9 & 3.62 & 8.90 & 42.1 & 256.8 & 207.2 & 1.240 & 0.791 \\
2.5 & $67^{\circ}$ & 42.2 & 3.56 & 8.75 & 41.4 & 251.1 & 202.6 & 1.240 & 0.765 \\
2.7 & $67^{\circ}$ & 41.5 & 3.50 & 8.61 & 40.7 & 245.7 & 198.3 & 1.240 & 0.739 \\
\hline 2.31 & $71^{\circ}$ & 42.3 & 3.57 & 9.02 & 41.5 & 260.8 & 204.5 & 1.276 & 0.770 \\
2.5 & $71^{\circ}$ & 41.6 & 3.51 & 8.87 & 40.8 & 255.1 & 200.0 & 1.276 & 0.744 \\
2.7 & $71^{\circ}$ & 40.9 & 3.45 & 8.72 & 40.1 & 249.6 & 195.7 & 1.276 & 0.719 \\
\hline
\end{tabular}

Table 5: CMFV predictions for various quantities as functions of $S(v)$ and $\gamma$. The four elements of the $C K M$ matrix are in units of $10^{-3}, F_{B_{s}} \sqrt{\hat{B}_{B_{s}}}$ and $F_{B_{d}} \sqrt{\hat{B}_{B_{d}}}$ in units of $\mathrm{MeV}$ and $\mathcal{B}\left(B^{+} \rightarrow \tau^{+} \nu\right)$ in units of $10^{-4}$.
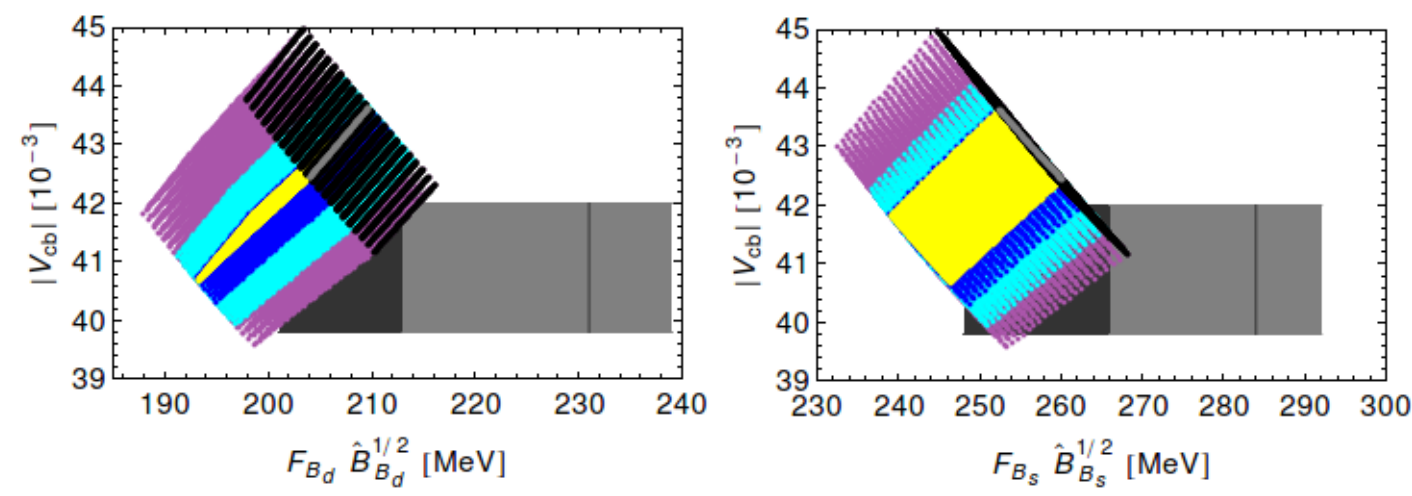

Figure 6: $\left|V_{c b}\right|$ versus $F_{B_{d}} \sqrt{\hat{B}_{B_{d}}}$ and $F_{B_{s}} \sqrt{\hat{B}_{B_{s}}}$ for $\gamma \in\left[63^{\circ}, 71^{\circ}\right]$. The yellow region corresponds to $S(v) \in[2.31,2.8], \eta_{c c}=1.87, \eta_{c t}=0.496$. In the purple region we include the errors in $\eta_{c c, c t}: S(v) \in[2.31,2.8], \eta_{c c} \in[1.10,2.64], \eta_{c t} \in[0.451,0.541]$. In the cyan region we use instead the reduced error of $\eta_{c c}$ as in $E q$. 81): $S(v) \in[2.31,2.8]$, $\eta_{c c} \in[1.49,1.91], \eta_{c t} \in[0.451,0.541]$. In the blue region we fix $\eta_{c t}$ to its central value: $S(v) \in[2.31,2.8], \eta_{c c} \in[1.49,1.91], \eta_{c t}=0.496$. To test the $S M$ we include the black region for fixed $S(v)=S_{0}\left(x_{t}\right)=2.31$ and $\eta_{c c, c t}$ as in the purple region. The gray line within the black $S M$ region corresponds to $\eta_{c c}=1.87$ and $\eta_{c t}=0.496$. Dark (light) gray box: $1 \sigma$ range of $F_{B_{d}} \sqrt{\hat{B}_{B_{d}}}, F_{B_{s}} \sqrt{\hat{B}_{B_{s}}}$ and $\left|V_{c b}\right|$ as given in Table 1 and (36), respectively. The vertical dark gray lines indicate where the dark gray boxes end. 

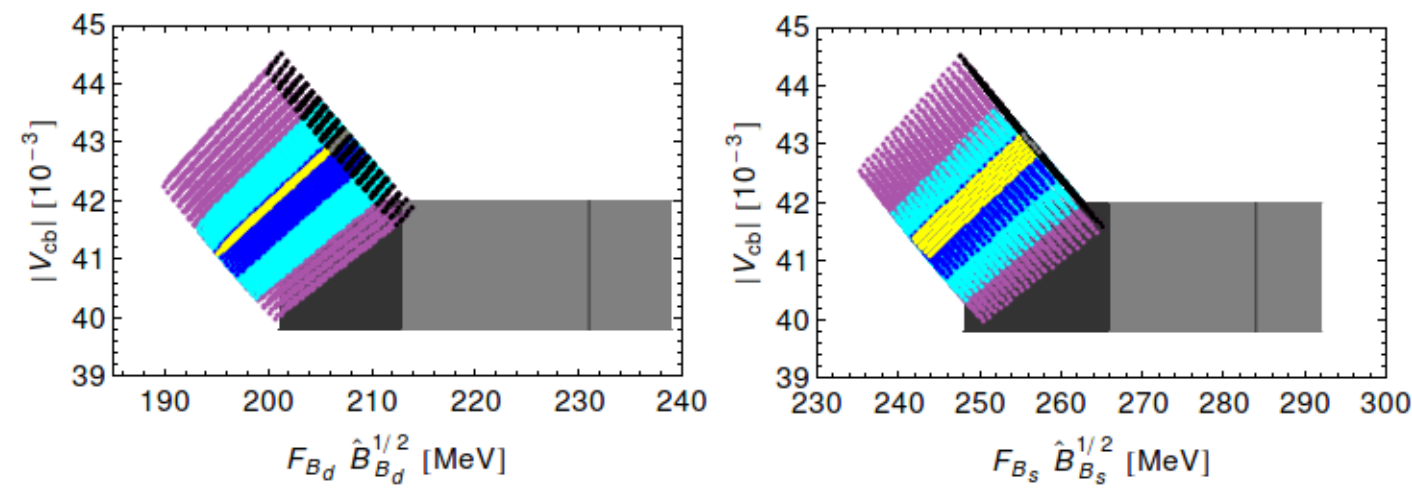

Figure 7: $\left|V_{c b}\right|$ versus $F_{B_{d}} \sqrt{\hat{B}_{B_{d}}}$ and $F_{B_{s}} \sqrt{\hat{B}_{B_{s}}}$ as in Fig. 6. but for $\gamma=(67 \pm 1)^{\circ}$.

\subsubsection{HDM with MFV and Flavour Blind Phases $\left(2 \mathrm{HDM}_{\overline{\mathrm{MFV}}}\right)$}

In view of our discussion above, this model [84 has in principle a better chance to remove simultaneously the anomalies in question than CMFV models but as we will soon see it approaches this problem in a different manner. The basic new features in $2 \mathrm{HDM}_{\overline{\mathrm{MFV}}}$ relative to $\mathrm{CMFV}$ are:

- The presence of flavour blind phases (FBPs) in this MFV framework modifies through their interplay with the standard CKM flavour violation the usual characteristic relations of the CMFV framework. In particular the mixing induced CP asymmetries $S_{\psi K_{S}}$ and $S_{\psi \phi}$ take the form known from non-MFV frameworks like LHT, RSc and SM4 as given in (67).

- The FBPs in the $2 \mathrm{HDM}_{\overline{\mathrm{MFV}}}$ can appear both in Yukawa interactions and in the Higgs potential. While in [84] only the case of FBPs in Yukawa interactions has been considered, in [140] these considerations have been extended to include also the FBPs in the Higgs potential. The two flavour-blind CPV mechanisms can be distinguished through the correlation between $S_{\psi K_{S}}$ and $S_{\psi \phi}$ that is strikingly different if only one of them is relevant. In fact the relation between generated new phases are very different in each case:

$$
\varphi_{B_{d}}=\frac{m_{d}}{m_{s}} \varphi_{B_{s}} \quad \text { and } \quad \varphi_{B_{d}}=\varphi_{B_{s}}
$$

for FBPs in Yukawa couplings and Higgs potential, respectively.

- New local operators are generated through the contributions of tree level heavy Higgs exchanges which also implies modified structure of flavour violation relatively to CMFV.

- Sizable FBPs, necessary to explain possible sizable non-standard CPV effects in $B_{s}$ mixing could, in principle, be forbidden by the upper bounds on EDMs of the 
neutron and the atoms. This question has been addressed in [140] and it has been shown that even for $S_{\psi \phi}=\mathcal{O}(1)$, this model still satisfied these bounds.

It is not our goal to describe the phenomenology of this model here in details as such details can be found in 84, 140]. Moreover a review appeared in [73. We rather want to emphasize that the model addresses the anomalies in question in a manner which differs profoundly from CMFV and thus a distinction between these two models can be already made on the basis of the data on $\Delta F=2$ processes.

Indeed in this model new contributions to $\varepsilon_{K}$ originating in tree level neutral Higgs exchanges are tiny being suppressed by small quark masses $m_{s, d}$. Consequently the correct value of $\varepsilon_{K}$ can only be obtained by choosing sufficiently large value of $\sin 2 \beta$ which corresponds to the large (inclusive) $\left|V_{u b}\right|$ scenario. If the formula (52) is used this in turn implies, as seen in Table 4 , a value of $S_{\psi K_{S}}$ which is much larger than the data. However, in this model the interplay of the CKM phase with the flavour blind phases in Yukawa couplings and Higgs potential generates non-vanishing new phases $\varphi_{B_{q}}$ and the formulae in (67) instead of (52) should be used. The new phases can suppress $S_{\psi K_{S}}$ simultaneously enhancing uniquely the asymmetry $S_{\psi \phi}$.

Now while the rate of the suppression of $S_{\psi K_{S}}$ for a given $S_{\psi \phi}$ is much stronger if significant FBPs in the Higgs potential rather than in Yukawa couplings are at work, both mechanism share a very important property:

- The necessary suppression of $S_{\psi K_{S}}$ necessarily implies uniquely the enhancement of $S_{\psi \phi}$ so that this asymmetry is larger than in the SM and consequently has positive sign. Finding eventually $S_{\psi \phi}$ at the LHC to be negative would be a real problem for the $2 \mathrm{HDM}_{\overline{\mathrm{MFV}}}$.

Now $\varepsilon_{K}$ can only be made consistent in this model by properly choosing $\gamma$ and in particular $\left|V_{u b}\right|$ that has to be sufficiently large. The question then arises, whether simultaneously also $S_{\psi K_{S}}, S_{\psi \phi}$ and $\Delta M_{d, s}$ can be made consistent with the data. We find then 141:

- The removal of the $\varepsilon_{K}-S_{\psi K_{S}}$ anomaly, which proceeds through the negative phase $\varphi_{B_{d}}$, is only possible with the help of FBPs in the Higgs potential. This is achieved in the case of the full dominance of the $Q_{1,2}^{\mathrm{SLL}}$ operators as far as $\mathrm{CP}$-violating contributions are concerned. If these operators also dominate the $\mathrm{CP}$-conserving contributions two important properties follow:

$$
\varphi_{B_{d}}=\varphi_{B_{s}}, \quad C_{B_{s}}=C_{B_{d}} .
$$

The second of the equalities implies

$$
\left(\frac{\Delta M_{s}}{\Delta M_{d}}\right)_{2 \mathrm{HDM}_{\overline{\mathrm{MFV}}}}=\left(\frac{\Delta M_{s}}{\Delta M_{d}}\right)_{\mathrm{SM}} .
$$




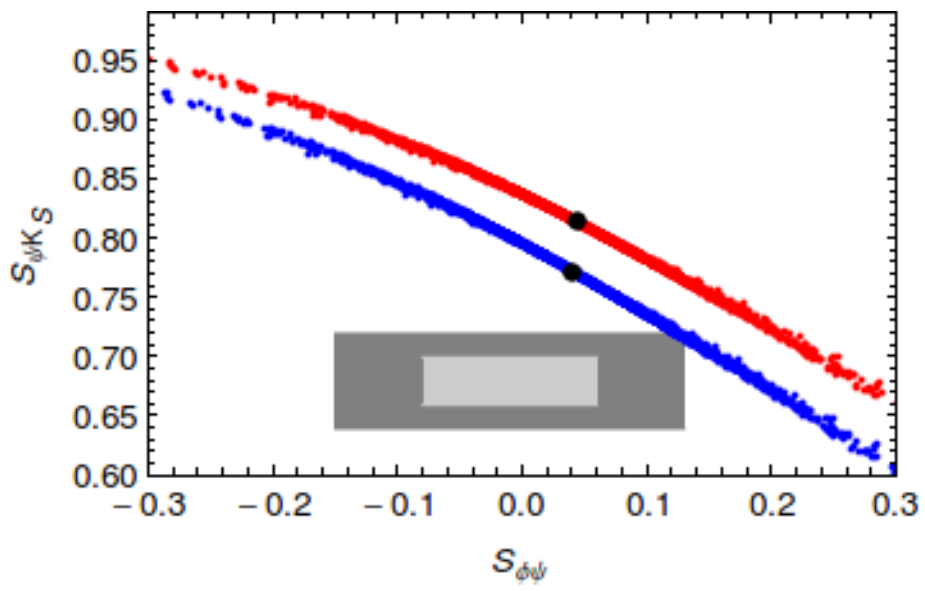

Figure 8: $S_{\psi K_{S}}$ vs. $S_{\psi \phi}$ in $2 \mathrm{HDM}_{\overline{\mathrm{MFV}}}$ for $\left|V_{u b}\right|=4.0 \cdot 10^{-3}$ (blue) and $\left|V_{u b}\right|=4.3 \cdot 10^{-3}$ (red). SM is represented by black points while $1 \sigma(2 \sigma)$ experimental range by the grey (dark grey) area [141].

This relation is known from models with CMFV but there $C_{B_{s}}=C_{B_{d}} \geq 1$. In $2 \mathrm{HDM}_{\overline{\mathrm{MFV}}}$ also $C_{B_{s}}=C_{B_{d}} \leq 1$ is possible. Moreover, the CMFV correlation between $\varepsilon_{K}$ and $\Delta M_{s, d}$ is absent and $\Delta M_{s, d}$ can be both suppressed and enhanced if necessary.

- A significant contribution of the operators $Q_{1,2}^{\mathrm{LR}}$ is unwanted as it spoils the relation (85) having much larger effect on $\Delta M_{s}$ than $\Delta M_{d}$. But as this contribution uniquely suppresses $\Delta M_{s}$ below its SM value, it could turn out relevant one day if the lattice results for hadronic matrix changed. This contribution cannot help in solving $\varepsilon_{K}-S_{\psi K_{S}}$ anomaly as its effect on the phase $\varphi_{B_{d}}$ is very small.

Thus at first sight at the qualitative level this model provides a better description of $\Delta F=2$ data than the $\mathrm{SM}$ and models with CMFV. Yet, here comes a possible difficulty. As shown in Fig. 8 the size of $\varphi_{B_{d}}$ that is necessary to obtain simultaneously good agreement with the data on $\varepsilon_{K}$ and $S_{\psi K_{S}}$ implies in turn $S_{\psi \phi} \geq 0.15$ which is $2 \sigma$ away from the LHCb central value in (59).

In summary $2 \mathrm{HDM}_{\overline{\mathrm{MFV}}}$ is from the point of view of $\Delta F=2$ observables in a reasonable shape. Yet, finding in the future that nature chooses a negative value of $S_{\psi \phi}$ and/or small (exclusive) value of $\left|V_{u b}\right|$ would practically rule out $2 \mathrm{HDM}_{\overline{\mathrm{MFV}}}$. Also a decrease of the experimental error on $S_{\psi \phi}$ without the change of its central value would be problematic for this model.

We are looking forward to improved experimental data and improved lattice calculations to find out whether this simple model can satisfactorily describe the data on $\Delta F=2$ observables. 


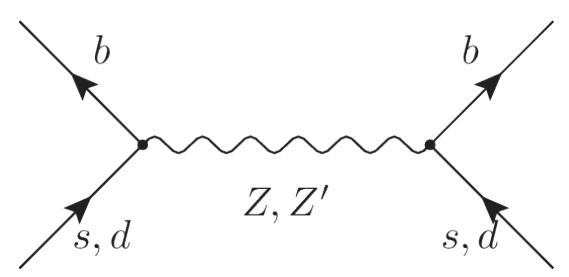

Figure 9: Tree-level flavour-changing $Z, Z^{\prime}$ contribution to $B_{d}^{0}-\bar{B}_{d}^{0}$ and $B_{s}^{0}-\bar{B}_{s}^{0}$ mixing.

\subsubsection{Tree-Level Gauge Boson Exchanges}

We will next investigate what a neutral gauge boson tree level exchange can contribute to this discussion. For the neutral gauge boson $Z^{\prime}$ contribution as shown in Fig. 9 one has generally 40,93

$$
\begin{aligned}
\left(M_{12}^{\star}\right)_{Z^{\prime}}^{b q}= & \frac{\left(\Delta_{L}^{b q}\left(Z^{\prime}\right)\right)^{2}}{2 M_{Z^{\prime}}^{2}} C_{1}^{\mathrm{VLL}}\left(\mu_{Z^{\prime}}\right)\left\langle Q_{1}^{\mathrm{VLL}}\left(\mu_{Z^{\prime}}\right)\right\rangle+\frac{\left(\Delta_{R}^{b q}\left(Z^{\prime}\right)\right)^{2}}{2 M_{Z^{\prime}}^{2}} C_{1}^{\mathrm{VRR}}\left(\mu_{Z^{\prime}}\right)\left\langle Q_{1}^{\mathrm{VLL}}\left(\mu_{Z^{\prime}}\right)\right\rangle \\
& +\frac{\Delta_{L}^{b q}\left(Z^{\prime}\right) \Delta_{R}^{b q}\left(Z^{\prime}\right)}{M_{Z^{\prime}}^{2}}\left[C_{1}^{\mathrm{LR}}\left(\mu_{Z^{\prime}}\right)\left\langle Q_{1}^{\mathrm{LR}}\left(\mu_{Z^{\prime}}\right)\right\rangle+C_{2}^{\mathrm{LR}}\left(\mu_{Z^{\prime}}\right)\left\langle Q_{2}^{\mathrm{LR}}\left(\mu_{Z^{\prime}}\right)\right\rangle\right],
\end{aligned}
$$

where including NLO QCD corrections [93]

$$
\begin{aligned}
C_{1}^{\mathrm{VLL}}\left(\mu_{Z^{\prime}}\right)=C_{1}^{\mathrm{VRR}}\left(\mu_{Z^{\prime}}\right) & =1+\frac{\alpha_{s}}{4 \pi}\left(-2 \log \frac{M_{Z^{\prime}}^{2}}{\mu_{Z^{\prime}}^{2}}+\frac{11}{3}\right), \\
C_{1}^{\mathrm{LR}}\left(\mu_{Z^{\prime}}\right) & =1+\frac{\alpha_{s}}{4 \pi}\left(-\log \frac{M_{Z^{\prime}}^{2}}{\mu_{Z^{\prime}}^{2}}-\frac{1}{6}\right), \\
C_{2}^{\mathrm{LR}}\left(\mu_{Z^{\prime}}\right) & =\frac{\alpha_{s}}{4 \pi}\left(-6 \log \frac{M_{Z^{\prime}}^{2}}{\mu_{Z^{\prime}}^{2}}-1\right) .
\end{aligned}
$$

Here $\left\langle Q_{i}^{a}\left(\mu_{Z^{\prime}}\right)\right\rangle$ are the matrix elements of operators evaluated at the matching scale. Their $\mu_{Z^{\prime}}$ dependence is canceled by the one of of $C_{i}^{a}\left(\mu_{Z^{\prime}}\right)$ so that $M_{12}$ does not depend on $\mu_{Z^{\prime}}$. The values of $\left\langle Q_{i}^{a}\left(\mu_{Z^{\prime}}\right)\right\rangle$ for $\mu_{H}=\mu_{Z^{\prime}}=1 \mathrm{TeV}$ can be found in Table 2. In the case of the $K$ system the indices $b q$ should be replaced by $s d$. The Wilson coefficients listed above remain unchanged and the relevant hadronic matrix elements are also collected in Table 2. If tree-level $Z$-boson exchanges are considered the matrix elements in Table 3 should be used, $M_{\mu_{Z^{\prime}}} \rightarrow M_{Z}$ and $\mu_{Z^{\prime}} \rightarrow m_{t}\left(m_{t}\right)$.

In the case of VLL and VRR operators it is more convenient to incorporated NP effects as shifts in the one-loop functions $S(v)$. These shifts, denoted by $[\Delta S(M)]_{\mathrm{VLL}}$ and $[\Delta S(M)]_{\mathrm{VRR}}$ have been calculated in [41] and are given as follows

$$
\left[\Delta S\left(B_{q}\right)\right]_{\mathrm{VLL}}=\left[\frac{\Delta_{L}^{b q}\left(Z^{\prime}\right)}{\lambda_{t}^{(q)}}\right]^{2} \frac{4 \tilde{r}}{M_{Z^{\prime}}^{2} g_{\mathrm{SM}}^{2}}, \quad[\Delta S(K)]_{\mathrm{VLL}}=\left[\frac{\Delta_{L}^{s d}\left(Z^{\prime}\right)}{\lambda_{t}^{(K)}}\right]^{2} \frac{4 \tilde{r}}{M_{Z^{\prime}}^{2} g_{\mathrm{SM}}^{2}},
$$


where

$$
g_{\mathrm{SM}}^{2}=4 \frac{G_{F}}{\sqrt{2}} \frac{\alpha}{2 \pi \sin ^{2} \theta_{W}}=1.78137 \times 10^{-7} \mathrm{GeV}^{-2} .
$$

Here $\tilde{r}=0.985, \tilde{r}=0.965, \tilde{r}=0.953$ and $\tilde{r}=0.925$ for $M_{Z^{\prime}}=1,2,3,10 \mathrm{TeV}$, respectively. $[\Delta S(M)]_{\mathrm{VRR}}$ is then found from the formula above by simply replacing $\mathrm{L}$ by R. For the case of tree-level $Z$ exchanges $\tilde{r}=1.068$.

For a qualitative discussion it is sufficient to set the Wilson coefficients to the LO values. Then

$$
\left(M_{12}^{\star}\right)_{Z^{\prime}}=\left(\frac{\left(\Delta_{L}^{s d}\left(Z^{\prime}\right)\right)^{2}}{2 M_{Z^{\prime}}^{2}}+\frac{\left(\Delta_{R}^{s d}\left(Z^{\prime}\right)\right)^{2}}{2 M_{Z^{\prime}}^{2}}\right)\left\langle Q_{1}^{\mathrm{VLL}}\left(\mu_{Z^{\prime}}\right)\right\rangle+\frac{\Delta_{L}^{s d}\left(Z^{\prime}\right) \Delta_{R}^{s d}\left(Z^{\prime}\right)}{M_{Z^{\prime}}^{2}}\left\langle Q_{1}^{\mathrm{LR}}\left(\mu_{Z^{\prime}}\right)\right\rangle
$$

with analogous expressions for other meson systems. Now as seen in Table 2 model independently

$$
\left\langle Q_{1}^{\mathrm{VLL}}\left(\mu_{Z^{\prime}}\right)\right\rangle>0, \quad\left\langle Q_{1}^{\mathrm{LR}}\left(\mu_{Z^{\prime}}\right)\right\rangle<0, \quad\left|\left\langle Q_{1}^{\mathrm{LR}}\left(\mu_{Z^{\prime}}\right)\right\rangle\right| \gg\left|\left\langle Q_{1}^{\mathrm{VLL}}\left(\mu_{Z^{\prime}}\right)\right\rangle\right|,
$$

which has an impact on the signs and size of the couplings $\Delta_{L, R}\left(Z^{\prime}\right)$ if these contributions should remove the anomalies in the data.

The outcome for the phenomenology depends on whether $\Delta_{L}$ and $\Delta_{R}$ are of comparable size or if one of them is dominant and whether they are real or complex quantities. Moreover these properties can be different for different meson systems. Evidently we have here in mind the scenarios LHS, RHS, LRS and ALRS of Section 2, Moreover, one has to distinguish between the Scenario 1 (S1) and Scenario 2 (S2) for $\left|V_{u b}\right|$, so that generally one deals with LHS1, LHS2 and similarly for RHS, LRS and ALRS.

As expected with these new contributions without any particular structure of the $\Delta_{L, R}$ couplings all tensions within the SM in the $\Delta F=2$ transitions can be removed in many ways and it will be important to investigate in the next steps which of them are also consistent with other constraints and which ones remove simultaneously other tensions, that are already present or will be generated when the data and lattice results improve in the future.

In concrete BSM models the couplings $\Delta_{L, R}^{i j}$, corresponding to different meson systems, could be related to each other as they may depend on the same fundamental parameters of an underlying theory. For instance in the minimal 3-3-1 model, analyzed recently in $[41,47]$, the flavour violating couplings $\Delta_{L}^{s d}\left(Z^{\prime}\right), \Delta_{L}^{b d}\left(Z^{\prime}\right)$ and $\Delta_{L}^{b s}\left(Z^{\prime}\right)$ depend on two mixing angles and two complex phases, instead of six parameters, which implies correlations between observables in different meson systems (see also Sec. 6.5.1).

A very detailed analysis of $B_{d, s}^{0}-\bar{B}_{d, s}^{0}$ and $K^{0}-\bar{K}^{0}$ systems has been presented in $[40$ setting the CKM parameters as in (55) and all the other input at the central values in Table 1 except that in [40] the input in (36) has been used. As the latter values are consistent with the present ones, in order to take partially hadronic and experimental uncertainties into account we will still present here the results of [40]. Moreover as in 
the latter paper we require that values of observables in question satisfy the following constraints

$$
\begin{gathered}
16.9 / \mathrm{ps} \leq \Delta M_{s} \leq 18.7 / \mathrm{ps}, \quad-0.20 \leq S_{\psi \phi} \leq 0.20 \\
0.48 / \mathrm{ps} \leq \Delta M_{d} \leq 0.53 / \mathrm{ps}, \quad 0.64 \leq S_{\psi K_{S}} \leq 0.72 . \\
0.75 \leq \frac{\Delta M_{K}}{\left(\Delta M_{K}\right)_{\mathrm{SM}}} \leq 1.25, \quad 2.0 \times 10^{-3} \leq\left|\varepsilon_{K}\right| \leq 2.5 \times 10^{-3} .
\end{gathered}
$$

The larger uncertainty for $\varepsilon_{K}$ than $\Delta M_{s, d}$ signals its strong $\left|V_{c b}\right|^{4}$ dependence. $\Delta M_{K}$ has even larger uncertainty because of potential long distance uncertainties. When using the constraint from $S_{\psi \phi}$ and $S_{\psi K_{S}}$ we take into account that only mixing phases close to their SM value are allowed by the data thereby removing some discrete ambiguities.

Parametrizing the different flavour violating couplings of $Z^{\prime}$ to quarks as follows

$$
\Delta_{L}^{b s}\left(Z^{\prime}\right)=-\tilde{s}_{23} e^{-i \delta_{23}}, \quad \Delta_{L}^{b d}\left(Z^{\prime}\right)=\tilde{s}_{13} e^{-i \delta_{13}}, \quad \Delta_{L}^{s d}\left(Z^{\prime}\right)=-\tilde{s}_{12} e^{-i \delta_{12}},
$$

it was possible to find the allowed oases in the spaces $\left(\tilde{s}_{i j}, \delta_{i j}\right)$ used to describe $Z^{\prime}$ effects in each system. The minus sign is introduced to cancel the one in $V_{t s}$.

In the case of $B_{s}^{0}-\bar{B}_{s}^{0}$ system the result of this search for $M_{Z^{\prime}}=1 \mathrm{TeV}$ and LHS1 scenario is shown in Fig. 10. The red regions correspond to the allowed ranges for $\Delta M_{s}$, while the blue ones to the corresponding ranges for $S_{\psi \phi}$. The overlap between red and blue regions (light blue and purple) identifies the oases we were looking for. We observe that the requirement of suppression of $\Delta M_{s}$ implies $\tilde{s}_{23} \neq 0$. As this system is immune to the value of $\left|V_{u b}\right|$ the same results are obtained for LHS2.

We note that for each oasis with a given $\delta_{23}$ there is another oasis with $\delta_{23}$ shifted by $180^{\circ}$ but the range for $\tilde{s}_{23}$ is unchanged. This discrete ambiguity results from the fact that $\Delta M_{s}$ and $S_{\psi \phi}$ are governed by $2 \delta_{23}$. This ambiguity can be resolved by other observables discussed in the next steps. The colour coding for the allowed oases, blue and purple for oasis with small and large $\delta_{23}$, respectively, will be useful in this context.

The corresponding oases for $B_{d}^{0}-\bar{B}_{d}^{0}$ and $K^{0}-\bar{K}^{0}$ systems are shown in Figs. 11 and 12, respectively. We note that now the results depend on whether LHS1 or LHS2 considered. Moreover in accordance with the quality of the constraints in (94)-(96), the allowed oases in the $B_{d}^{0}-\bar{B}_{d}^{0}$ system are smaller than in the $B_{s}^{0}-\bar{B}_{s}^{0}$ system, while they are larger in the $K^{0}-\bar{K}^{0}$ system. The colour coding for allowed oases in these figures will be useful to monitor the following steps in which rare $B_{d}$ and $K$ decays will be discussed and the distinction between the two allowed oases in each case will be possible.

In [40] also the allowed oases in scenarios RHS, LRS and ALRS have been considered. We summarize here the main results and refer for details to this paper: 


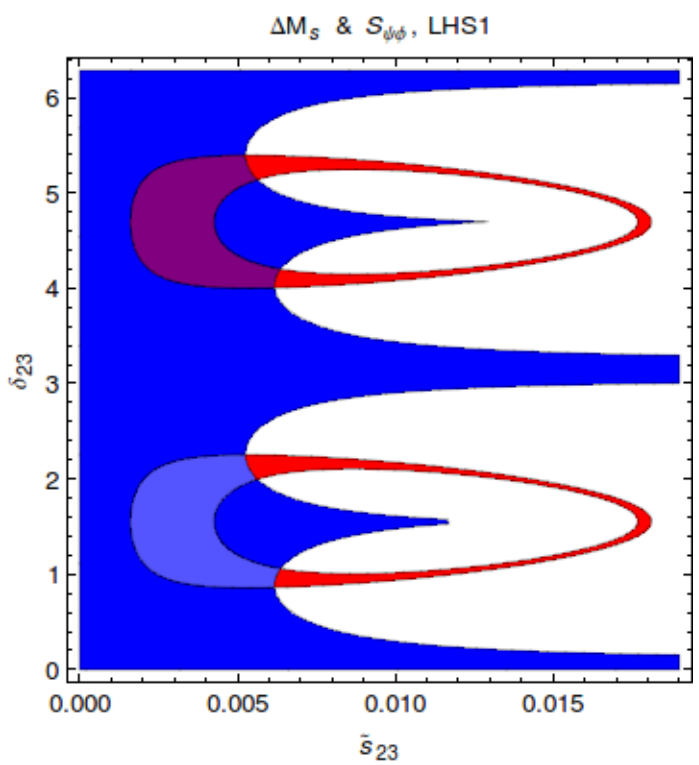

Figure 10: Ranges for $\Delta M_{s}$ (red region) and $S_{\psi \phi}$ (blue region) for $M_{Z^{\prime}}=1$ TeV in LHS1 satisfying the bounds in Eq. (94).
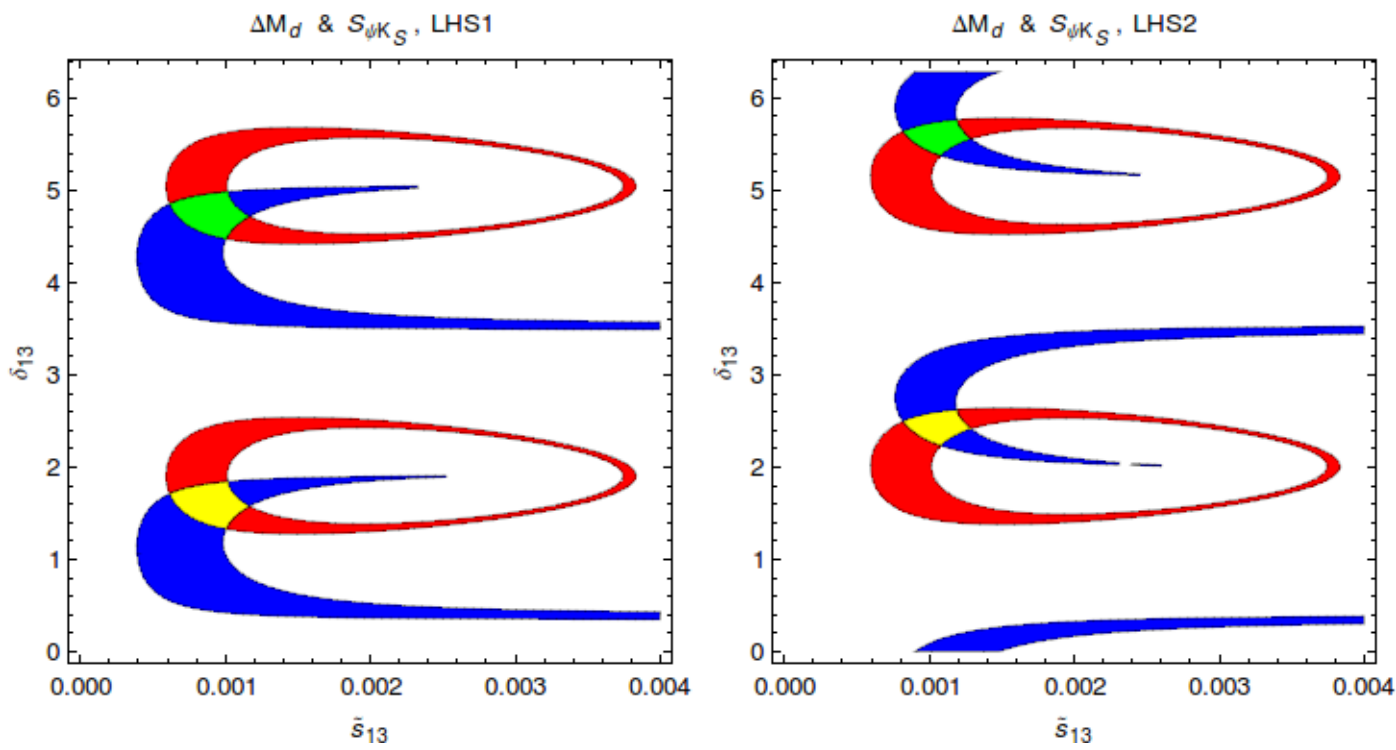

Figure 11: Ranges for $\Delta M_{d}$ (red region) and $S_{\psi K_{S}}$ (blue region) for $M_{Z^{\prime}}=1$ TeV in LHS1 (left) and LHS2 (right) satisfying the bounds in Eq. 95.).

- In the case of RHS scenarios the oases in the space of parameters related to RH currents are precisely the same as those just discussed for LHS scenarios, except that the parameters $\tilde{s}_{i j}$ and $\delta_{i j}$ parametrize now RH and not LH currents. Yet, as 

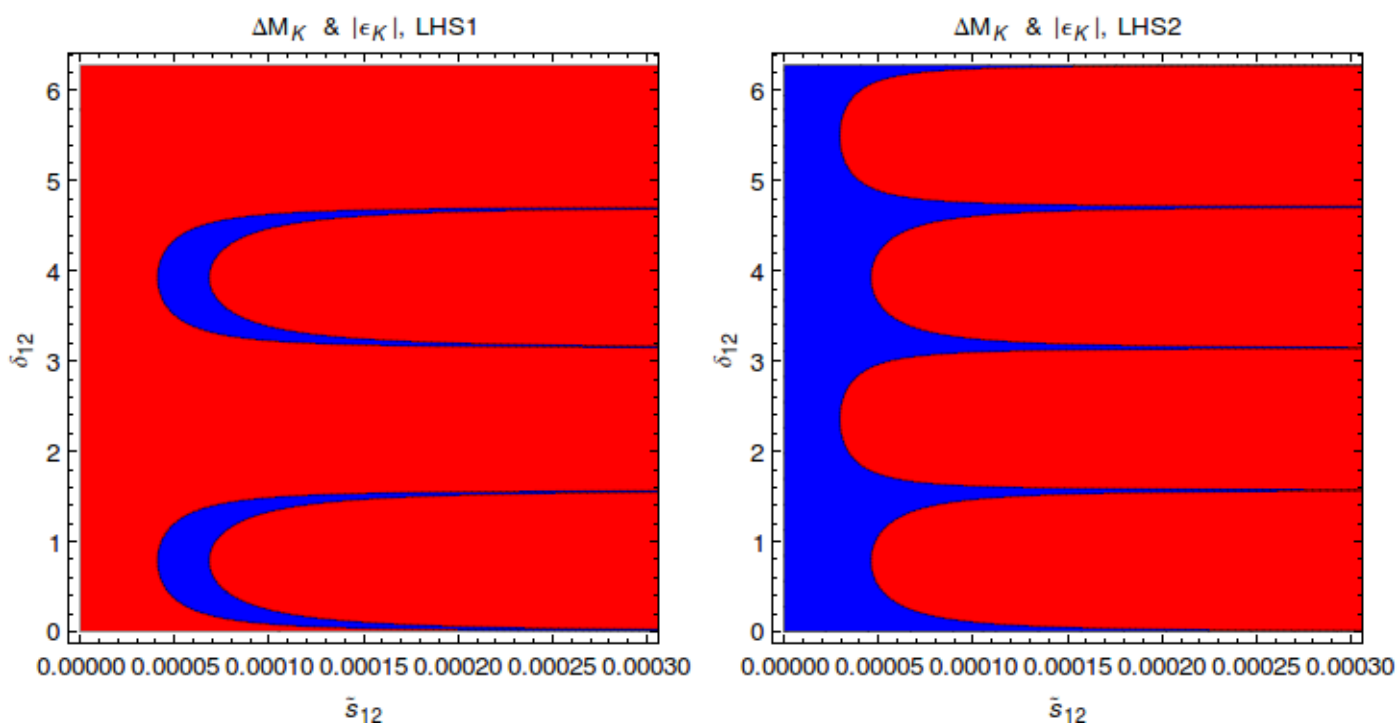

Figure 12: Ranges for $\Delta M_{K}$ (red region) and $\varepsilon_{K}$ (blue region) (LHS1: left, LHS2: right) for $M_{Z^{\prime}}=1$ TeV satisfying the bounds in Eq. (96).

we will see in the next steps in the case of $\Delta F=1$ observables some distinction between $\mathrm{LH}$ and RH currents will be possible.

- In the LRS scenarios NP contributions to $\Delta F=2$ observables are dominated by new LR operators, whose contributions are enhanced through renormalization group effects relative to LL and RR operators and in the case of $\varepsilon_{K}$ also through chirally enhanced hadronic matrix elements. Consequently the oases will differ from the previous ones and typically the corresponding $\tilde{s}_{i j}$ will be smaller in order to obtain agreement with the data. The results can be found in Figs. 13-15 of [40]. In order to understand these plots one should recall that the matrix element of the dominant $Q_{1}^{\mathrm{LR}}$ operator has the sign opposite to SM operators. Therefore, in the case of $B_{s, d}^{0}-\bar{B}_{s, d}^{0}$ systems this operator naturally suppresses $\Delta M_{s}$ and $\Delta M_{d}$ with the phase $\delta_{23}$ and $\delta_{13}$ shifted down by roughly $90^{\circ}$ relatively to the LHS scenarios. We illustrate this in Fig. 13 for LRS1 scenario. These plots should be compared with the one in Fig. 10 and in the left panel of Fig. 11, respectively.

- The allowed oases in ALR scenarios have the same phase structure as in LHS scenarios because the contributions of the dominant LR operators have the same sign as SM contributions. Only the allowed values of $\tilde{s}_{i j}$ are smaller because of larger hadronic matrix elements than in the LHS case.

The implications of these results for rare decays will be presented in the next steps. 

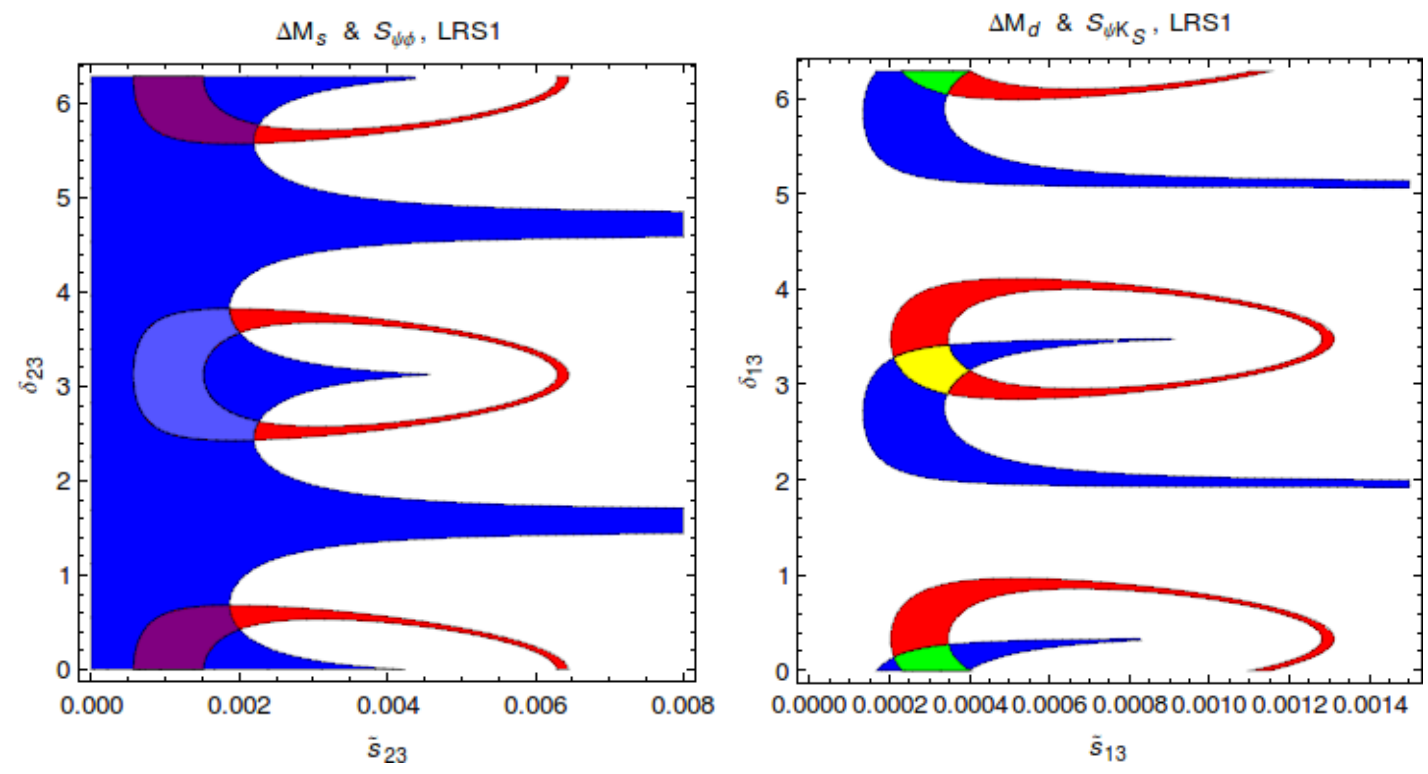

Figure 13: Ranges for $\Delta M_{s}$ and $S_{\psi \phi}$ (left) and $\Delta M_{d}$ and $S_{\psi K_{S}}$ (right) for $M_{Z^{\prime}}=1 \mathrm{TeV}$ in LRS1 satisfying the bounds in Eq. 94) and Eq. 95).

\subsubsection{Tree-Level Scalar Exchanges}

We next turn our attention to tree-level heavy scalar exchanges to $\Delta F=2$ transitions (see Fig. 14). Here one finds [44,93

$$
\begin{aligned}
\left(M_{12}^{\star}\right)_{H}= & -\frac{\left(\Delta_{L}^{s d}(H)\right)^{2}}{2 M_{H}^{2}}\left[C_{1}^{\mathrm{SLL}}\left(\mu_{H}\right)\left\langle Q_{1}^{\mathrm{SLL}}\left(\mu_{H}\right)\right\rangle+C_{2}^{\mathrm{SLL}}\left(\mu_{H}\right)\left\langle Q_{2}^{\mathrm{SLL}}\left(\mu_{H}\right)\right\rangle\right] \\
& -\frac{\left(\Delta_{R}^{s d}(H)\right)^{2}}{2 M_{H}^{2}}\left[C_{1}^{\mathrm{SRR}}\left(\mu_{H}\right)\left\langle Q_{1}^{\mathrm{SRR}}\left(\mu_{H}\right)\right\rangle+C_{2}^{\mathrm{SRR}}\left(\mu_{H}\right)\left\langle Q_{2}^{\mathrm{SRR}}\left(\mu_{H}\right)\right\rangle\right] \\
& -\frac{\Delta_{L}^{s d}(H) \Delta_{R}^{s d}(H)}{M_{H}^{2}}\left[C_{1}^{\mathrm{LR}}\left(\mu_{H}\right)\left\langle Q_{1}^{\mathrm{LR}}\left(\mu_{H}\right)\right\rangle+C_{2}^{\mathrm{LR}}\left(\mu_{H}\right)\left\langle Q_{2}^{\mathrm{LR}}\left(\mu_{H}\right)\right\rangle\right],
\end{aligned}
$$

where including NLO QCD corrections [93]

$$
\begin{gathered}
C_{1}^{\mathrm{SLL}}(\mu)=C_{1}^{\mathrm{SRR}}(\mu)=1+\frac{\alpha_{s}}{4 \pi}\left(-3 \log \frac{M_{H}^{2}}{\mu^{2}}+\frac{9}{2}\right), \\
C_{2}^{\mathrm{SLL}}(\mu)=C_{2}^{\mathrm{SRR}}(\mu)=\frac{\alpha_{s}}{4 \pi}\left(-\frac{1}{12} \log \frac{M_{H}^{2}}{\mu^{2}}+\frac{1}{8}\right), \\
C_{1}^{\mathrm{LR}}(\mu)=-\frac{3}{2} \frac{\alpha_{s}}{4 \pi},
\end{gathered}
$$




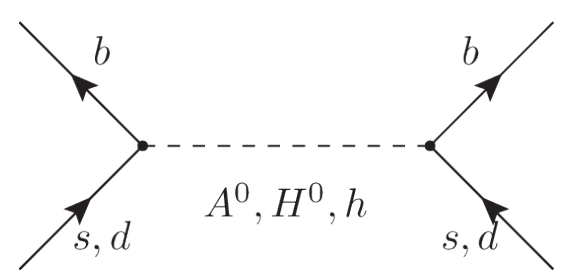

Figure 14: Tree-level flavour-changing $A^{0}, H^{0}, h$ contribution to $B_{d}^{0}-\bar{B}_{d}^{0}$ and $B_{s}^{0}-$ $\bar{B}_{s}^{0}$ mixing.

$$
C_{2}^{\mathrm{LR}}(\mu)=1-\frac{\alpha_{s}}{4 \pi} .
$$

Note that the scalar contributions to $C_{1,2}^{\mathrm{LR}}$ differ from the ones from gauge bosons. The relevant matrix elements can again be found in Tables 2 and 3 for tree-level heavy scalar and SM Higgs contributions. In the later case $M_{H}=M_{h}$ with $h$ standing for the SM Higgs.

For our qualitative discussion it is sufficient to set the Wilson coefficients to the LO values. Then

$$
\left(M_{12}^{\star}\right)_{H}=-\left(\frac{\left(\Delta_{L}^{s d}(H)\right)^{2}}{2 M_{H}^{2}}+\frac{\left(\Delta_{R}^{s d}(H)\right)^{2}}{2 M_{H}^{2}}\right)\left\langle Q_{1}^{\mathrm{SLL}}\left(\mu_{H}\right)\right\rangle-\frac{\Delta_{L}^{s d}(H) \Delta_{R}^{s d}(H)}{M_{H}^{2}}\left\langle Q_{2}^{\mathrm{LR}}\left(\mu_{H}\right)\right\rangle
$$

with analogous expressions for other meson systems. Now as seen in Table 2 model independently

$$
\left\langle Q_{1}^{\mathrm{SLL}}\left(\mu_{H}\right)\right\rangle<0, \quad\left\langle Q_{2}^{\mathrm{LR}}\left(\mu_{H}\right)\right\rangle>0, \quad\left|\left\langle Q_{2}^{\mathrm{LR}}\left(\mu_{H}\right)\right\rangle\right| \gg\left|\left\langle Q_{1}^{\mathrm{VLL}}\left(\mu_{H}\right)\right\rangle\right|,
$$

which has an impact on the signs and size of the couplings $\Delta_{L, R}(H)$ if these contributions should remove the anomalies in the data.

Interestingly the signs of $\left\langle Q_{i}^{a}\right\rangle$ that are relevant in gauge boson and scalar cases are such that at the end it is not possible to distinguish these two cases on the basis of the signs of the couplings alone. On the other hand $\left\langle Q_{i}^{\mathrm{SLL}}\right\rangle$ are absent in the case of gauge boson exchanges and $\Delta_{L, R}\left(Z^{\prime}\right)$ and $\Delta_{L, R}(H)$ are generally different from each other so that some distinction will be possible when other decays will be taken into account in later steps. Otherwise, the qualitative comments made in the context of tree-level gauge boson exchanges can be repeated in this case.

Indeed as analyzed recently in [44] the phase structure of the allowed oases is identical to the one of the gauge boson case. As seen in the plots presented in this paper only the values of $\tilde{s}_{i j}$ change.

\subsubsection{Implications of $U(2)^{3}$ Symmetry}

Possibly the simplest solution to the problems of various models with MFV is to reduce the flavour symmetry $U(3)^{3}$ to $U(2)^{3} \sqrt{86} \sqrt{92}$. As pointed out in $[39$ in this case NP 


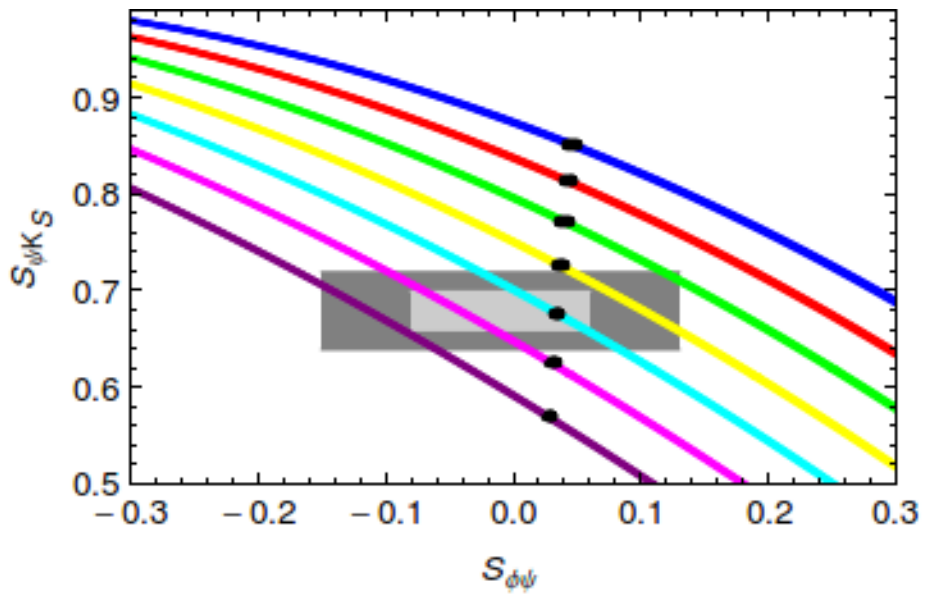

Figure 15: $S_{\psi K_{S}}$ vs. $S_{\psi \phi}$ in models with $U(2)^{3}$ symmetry for different values of $\left|V_{u b}\right|$ and $\gamma \in\left[58^{\circ}, 78^{\circ}\right]$. From top to bottom: $\left|V_{u b}\right|=0.0046$ (blue), 0.0043 (red), 0.0040 (green), 0.0037 (yellow), 0.0034 (cyan), 0.0031 (magenta), 0.0028 (purple). Light/dark gray: experimental $1 \sigma / 2 \sigma$ region.

effects in $\varepsilon_{K}$ and $B_{s, d}^{0}-\bar{B}_{s, d}^{0}$ are not correlated with each other so that the enhancement of $\varepsilon_{K}$ and suppression of $\Delta M_{s, d}$ can be achieved if necessary in principle for the values of $\left|V_{c b}\right|, F_{B_{s}} \sqrt{\hat{B}_{B_{s}}}$ and $F_{B_{d}} \sqrt{\hat{B}_{B_{d}}}$ in Table 1 or 36 .

In particular,

- NP effects in $\varepsilon_{K}$ are of CMFV type and $\varepsilon_{K}$ can only be enhanced. But because of the reduced flavour symmetry from $U(3)^{3}$ to $U(2)^{3}$ there is no correlation between $\varepsilon_{K}$ and $\Delta M_{s, d}$ which was problematic for CMFV models.

- In $B_{s, d}^{0}-\bar{B}_{s, d}^{0}$ system, the ratio $\Delta M_{s} / \Delta M_{d}$ is equal to the one in the SM and in good agreement with the data. But in view of new CP-violating phases $\varphi_{B_{d}}$ and $\varphi_{B_{s}}$ even in the presence of only SM operators, $\Delta M_{s, d}$ can be suppressed. But the $U(2)^{3}$ symmetry implies $\varphi_{B_{d}}=\varphi_{B_{s}}$ and consequently a triple $S_{\psi K_{S}}-S_{\psi \phi}-\left|V_{u b}\right|$ correlation which constitutes an important test of this NP scenario [39]. We show this correlation in Fig. 15 for $\gamma$ between $58^{\circ}$ and $78^{\circ}$. Note that this correlation is independent of the values of $F_{B_{s}} \sqrt{\hat{B}_{B_{s}}}$ and $F_{B_{d}} \sqrt{\hat{B}_{B_{d}}}$.

- As seen in this figure the important advantage of $U(2)^{3}$ models over $2 \mathrm{HDM}_{\overline{\mathrm{MFV}}}$ is that in the case of $S_{\psi \phi}$ being very small or even having opposite sign to SM prediction, this framework can survive with concrete prediction for $\left|V_{u b}\right|$.

It is of interest to see how the parameter space in tree-level gauge boson or scalar $\Delta F=2$ transitions is further constrained when the flavour $U(2)^{3}$ symmetry is imposed 

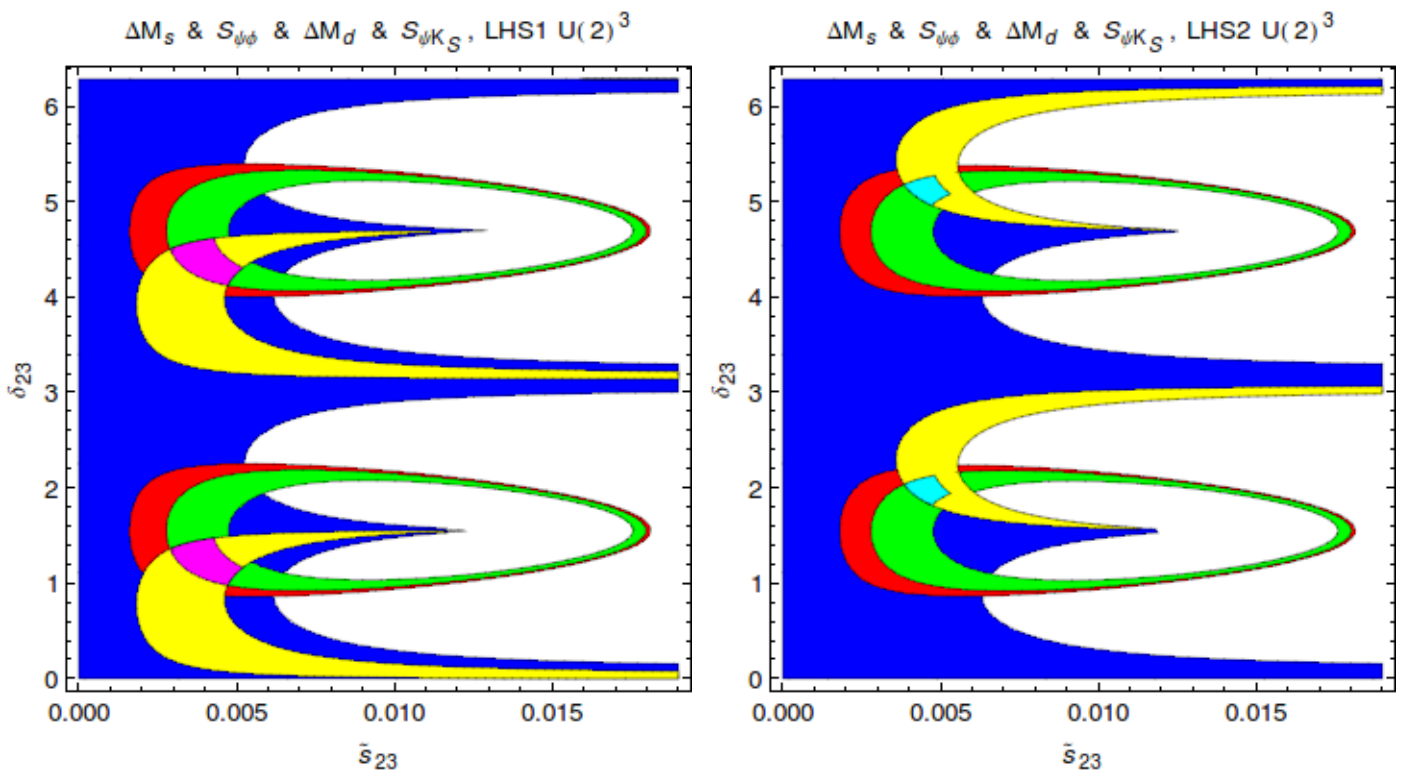

Figure 16: Ranges for $\Delta M_{s}$ (red region), $S_{\psi \phi}$ (blue region), $\Delta M_{d}$ (green region) and $S_{\psi K_{S}}$ (yellow region) for $M_{Z^{\prime}}=1 \mathrm{TeV}$ in LHS1 (left) and LHS2 (right) in the U(2) limit satisfying the bounds in Eq. 94) and 955). The overlap region of LHS1 (LHS2) is shown in magenta (cyan).

on the $Z^{\prime}$ or $H$ quark couplings. Indeed now the observables in $B_{d}$ and $B_{s}$ systems are correlated with each other due to the relations:

$$
\frac{\tilde{s}_{13}}{\left|V_{t d}\right|}=\frac{\tilde{s}_{23}}{\left|V_{t s}\right|}, \quad \delta_{13}-\delta_{23}=\beta-\beta_{s} .
$$

Thus, once the allowed oases in the $B_{d}$ system are fixed, the oases in $B_{s}$ system are determined. Moreover, all observables in both systems are described by only one real positive parameter and one phase, e.g. $\left(\tilde{s}_{23}, \delta_{23}\right)$.

The impact of $U(2)^{3}$ symmetry on tree level FCNCs due to gauge boson and scalar exchanges has been analyzed in [40] and [44], respectively. Again the phase structure in both cases is the same. Fig. 16 results from the combination of Figs. 10 and 11 using the $U(2)^{3}$ symmetry relations in $(105)$. We observe that in particular the $\left(\tilde{s}_{23}, \delta_{23}\right)$ oases are significantly reduced. Moreover the fact that the results in the $B_{d}$ system depend on whether LHS1 or LHS2 is considered is now transfered through the relations in (105) into the $B_{s}$ system. This is clearly seen in Fig. 16, in particular the final oases (cyan) in LHS2 are smaller than in LHS1 (magenta) due to the required shift of $S_{\psi K_{S}}$. The corresponding results in the scalar case can be found in Fig. 15 of [44]. It will be interesting to see what is the impact of the $U(2)^{3}$ symmetry on rare decays in the next steps. 


\subsection{Step 4: $\mathcal{B}\left(B_{s, d} \rightarrow \mu^{+} \mu^{-}\right)$and $\mathcal{B}\left(B_{s, d} \rightarrow \tau^{+} \tau^{-}\right)$}

\subsubsection{Preliminaries}

We now move to consider two superstars of rare $B$ decays: the decays $B_{s, d} \rightarrow \mu^{+} \mu^{-}$. We will also discuss $B_{s, d} \rightarrow \tau^{+} \tau^{-}$which could become superstars in the future. The particular interest in $B_{s, d} \rightarrow \mu^{+} \mu^{-}$is related to the fact that in the SM their branching ratios are not only loop and GIM suppressed as other rare decays in the SM. As the final state is purely leptonic and the initial state is a pseudoscalar the decays in question are strongly helicity suppressed in view of the smallness of $m_{\mu}$ and equally importantly do not receive photon-mediated one-loop contributions. As all these properties can be violated beyond the SM, these two decays are particularly suited for searching for NP being in addition theoretically very clean.

In the SM and in several of its extensions $\mathcal{B}\left(B_{s} \rightarrow \mu^{+} \mu^{-}\right)$is found in the ballpark of $(2-6) \cdot 10^{-9}$. As several model studies show this is the case of models in which these decays proceed through $Z$-penguin diagrams and tree-level neutral gauge boson exchanges. Larger values can be obtained in the presence of neutral heavy scalar and pseudoscalar exchanges in 2HDM models and Supersymmetry. Here these decays are governed by scalar and pseudoscalar penguins when the value of $\tan \beta$ is large. In certain models contributions from tree-level scalars and pseudoscalars can arise already at the fundamental level. Therefore a discovery of $\mathcal{B}\left(B_{s} \rightarrow \mu^{+} \mu^{-}\right)$at $\mathcal{O}\left(10^{-8}\right)$ would be a clear signal of NP, possibly related to such scalar and pseudoscalar exchanges [37]. Unfortunately, as we will see below, the most recent data from LHCb and CMS tell us that the nature does not allow us for a clear distinction between scalar, pseudoscalar and gauge boson contributions at least on the basis of the $\mathcal{B}\left(B_{s} \rightarrow \mu^{+} \mu^{-}\right)$alone. Either other observables related to the time-dependent rate of this decay have to be studied [43] or/and correlations with other observables have to be investigated. We will see explicit examples below. We refer also to 142, 143] where various virtues of these decays have been reviewed.

In order to discuss these issues we have to present the fundamental effective Hamiltonian relevant for these decays and other $b \rightarrow s \ell^{+} \ell^{-}$transitions, like $B \rightarrow K^{*} \ell^{+} \ell^{-}, B \rightarrow$ $K \ell^{+} \ell^{-}$and $B \rightarrow X_{s} \ell^{+} \ell^{-}$, which we will consider in Step 7 .

\subsubsection{Basic Formulae}

There are different conventions for operators [144 146] relevant for $b \rightarrow s \ell^{+} \ell^{-}$transitions and one has to be careful when using them along with the expressions for the branching ratios present in the literature. The effective Hamiltonian used here and in several recent papers is given as follows:

$$
\mathcal{H}_{\mathrm{eff}}(b \rightarrow s \ell \bar{\ell})=\mathcal{H}_{\mathrm{eff}}(b \rightarrow s \gamma)-\frac{4 G_{\mathrm{F}}}{\sqrt{2}} \frac{\alpha}{4 \pi} V_{t s}^{*} V_{t b} \sum_{i=9,10, S, P}\left[C_{i}(\mu) Q_{i}(\mu)+C_{i}^{\prime}(\mu) Q_{i}^{\prime}(\mu)\right]
$$


where

$$
\begin{aligned}
Q_{9} & =\left(\bar{s} \gamma_{\mu} P_{L} b\right)\left(\bar{\ell} \gamma^{\mu} \ell\right), & Q_{9}^{\prime} & =\left(\bar{s} \gamma_{\mu} P_{R} b\right)\left(\bar{\ell} \gamma^{\mu} \ell\right), \\
Q_{10} & =\left(\bar{s} \gamma_{\mu} P_{L} b\right)\left(\bar{\ell} \gamma^{\mu} \gamma_{5} \ell\right), & Q_{10}^{\prime} & =\left(\bar{s} \gamma_{\mu} P_{R} b\right)\left(\bar{\ell} \gamma^{\mu} \gamma_{5} \ell\right), \\
Q_{S} & =m_{b}\left(\bar{s} P_{R} b\right)(\bar{\ell} \ell), & Q_{S}^{\prime} & =m_{b}\left(\bar{s} P_{L} b\right)(\bar{\ell} \ell), \\
Q_{P} & =m_{b}\left(\bar{s} P_{R} b\right)\left(\bar{\ell} \gamma_{5} \ell\right), & Q_{P}^{\prime} & =m_{b}\left(\bar{s} P_{L} b\right)\left(\bar{\ell} \gamma_{5} \ell\right) .
\end{aligned}
$$

Here $\mathcal{H}_{\text {eff }}(b \rightarrow s \gamma)$ stands for the effective Hamiltonian for the $b \rightarrow s \gamma$ transition that involves the dipole operators (see Step 6). While we do not show explicitly the fourquark operators in (106) they are very important for decays considered in this step, in particular as far as QCD and electroweak corrections are concerned.

One should note the difference of ordering of flavours relatively to $\Delta F=2$ operators considered in the previous step. This will play a role as we discuss below (for example the relations of the couplings in (160) are useful when comparing $\Delta F=1$ and $\Delta F=$ 2 transitions). We neglect effects proportional to $m_{s}$ but keep $m_{s}$ and $m_{d}$ different from zero when they are shown explicitly. Analogous operators govern the $b \rightarrow d \ell^{+} \ell^{-}$ transitions, in particular the $B_{d} \rightarrow \mu^{+} \mu^{-}$decay.

Concentrating first on $B_{s} \rightarrow \mu^{+} \mu^{-}$, there are three observables which can be used to search for NP in these decays. These are

$$
\overline{\mathcal{B}}\left(B_{s} \rightarrow \mu^{+} \mu^{-}\right), \quad \mathcal{A}_{\Delta \Gamma}^{\mu \mu}, \quad S_{\mu \mu}^{s}
$$

Here $\overline{\mathcal{B}}\left(B_{s} \rightarrow \mu^{+} \mu^{-}\right)$is the usual branching ratio which includes $\Delta \Gamma_{s}$ effects pointed out in in [147 149]. Following [43] we will denote this branching ratio with a bar while the one without these effects without it. These two branching ratios are related through $147+149$

$$
\mathcal{B}\left(B_{s} \rightarrow \mu^{+} \mu^{-}\right)=r\left(y_{s}\right) \overline{\mathcal{B}}\left(B_{s} \rightarrow \mu^{+} \mu^{-}\right)
$$

where

$$
r\left(y_{s}\right) \equiv \frac{1-y_{s}^{2}}{1+\mathcal{A}_{\Delta \Gamma}^{\mu^{+} \mu^{-}} y_{s}}
$$

with 112

$$
y_{s} \equiv \tau_{B_{s}} \frac{\Delta \Gamma_{s}}{2}=0.062 \pm 0.009
$$

The observables $\mathcal{A}_{\Delta \Gamma}^{\mu \mu}$ and $S_{\mu \mu}^{s}$ can only be measured through time-dependent studies and appear in the time-dependent rate asymmetry as follows

$$
\frac{\Gamma\left(B_{s}^{0}(t) \rightarrow \mu^{+} \mu^{-}\right)-\Gamma\left(\bar{B}_{s}^{0}(t) \rightarrow \mu^{+} \mu^{-}\right)}{\Gamma\left(B_{s}^{0}(t) \rightarrow \mu^{+} \mu^{-}\right)+\Gamma\left(\bar{B}_{s}^{0}(t) \rightarrow \mu^{+} \mu^{-}\right)}=\frac{S_{\mu \mu}^{s} \sin \left(\Delta M_{s} t\right)}{\cosh \left(y_{s} t / \tau_{B_{s}}\right)+\mathcal{A}_{\Delta \Gamma}^{\mu \mu} \sinh \left(y_{s} t / \tau_{B_{s}}\right)}
$$


$\mathcal{A}_{\Delta \Gamma}^{\mu \mu}$ can be extracted from the untagged data sample, namely from the measurement of the effective lifetime, for which no distinction is made between initially present $B_{s}^{0}$ or $\bar{B}_{s}^{0}$ mesons. If tagging information is included, requiring the distinction between initially present $B_{s}^{0}$ or $\bar{B}_{s}^{0}$ mesons, a CP-violating asymmetry $S_{\mu \mu}^{s}$ can also be measured. Presently only $\overline{\mathcal{B}}\left(B_{s} \rightarrow \mu^{+} \mu^{-}\right)$is known experimentally but once $\mathcal{A}_{\Delta \Gamma}^{\mu \mu}$ will be extracted from time-dependent measurements, we will be able to obtain $\mathcal{B}\left(B_{s} \rightarrow \mu^{+} \mu^{-}\right)$directly from experiment as well. As emphasized and demonstrated in [43] $\mathcal{A}_{\Delta \Gamma}^{\mu \mu}$ and $S_{\mu \mu}^{s}$ provide additional information about possible NP which cannot be obtained on the basis of the branching ratio alone. In order to present the results for the trio in (108) in various models we have to express these observables in terms of the Wilson coefficients in the effective Hamiltonian in (106).

To this end one introduces first

$$
\begin{aligned}
& P \equiv \frac{C_{10}-C_{10}^{\prime}}{C_{10}^{\mathrm{SM}}}+\frac{m_{B_{s}}^{2}}{2 m_{\mu}} \frac{m_{b}}{m_{b}+m_{s}} \frac{C_{P}-C_{P}^{\prime}}{C_{10}^{\mathrm{SM}}} \equiv|P| e^{i \varphi_{P}} \\
& S \equiv \sqrt{1-\frac{4 m_{\mu}^{2}}{m_{B_{s}}^{2}}} \frac{m_{B_{s}}^{2}}{2 m_{\mu}} \frac{m_{b}}{m_{b}+m_{s}} \frac{C_{S}-C_{S}^{\prime}}{C_{10}^{\mathrm{SM}}} \equiv|S| e^{i \varphi_{S}},
\end{aligned}
$$

which carry the full information about dynamics in the decay. However, due to effects from $B_{s}^{0}-\bar{B}_{s}^{0}$ mixing, represented here by $y_{s}$, also the new phase $\varphi_{B_{s}}$ in $B_{s}^{0}-\bar{B}_{s}^{0}$ mixing will enter the expressions below.

One finds then three fundamental formulae $43,149,150$

$$
\begin{gathered}
\frac{\overline{\mathcal{B}}\left(B_{s} \rightarrow \mu^{+} \mu^{-}\right)}{\overline{\mathcal{B}}\left(B_{s} \rightarrow \mu^{+} \mu^{-}\right)_{\mathrm{SM}}}=\left[\frac{1+\mathcal{A}_{\Delta \Gamma}^{\mu \mu} y_{s}}{1+y_{s}}\right] \times\left(|P|^{2}+|S|^{2}\right) \\
=\left[\frac{1+y_{s} \cos \left(2 \varphi_{P}-2 \varphi_{B_{s}}\right)}{1+y_{s}}\right]|P|^{2}+\left[\frac{1-y_{s} \cos \left(2 \varphi_{S}-2 \varphi_{B_{s}}\right)}{1+y_{s}}\right]|S|^{2}, \\
\mathcal{A}_{\Delta \Gamma}^{\mu \mu}=\frac{|P|^{2} \cos \left(2 \varphi_{P}-2 \varphi_{B_{s}}\right)-|S|^{2} \cos \left(2 \varphi_{S}-2 \varphi_{B_{s}}\right)}{|P|^{2}+|S|^{2}} \\
S_{\mu \mu}^{s}=\frac{|P|^{2} \sin \left(2 \varphi_{P}-2 \varphi_{B_{s}}\right)-|S|^{2} \sin \left(2 \varphi_{S}-2 \varphi_{B_{s}}\right)}{|P|^{2}+|S|^{2}}
\end{gathered}
$$

where

$$
\begin{aligned}
& \overline{\mathcal{B}}\left(B_{s} \rightarrow \mu^{+} \mu^{-}\right)_{\mathrm{SM}}=\frac{1}{1-y_{s}} \mathcal{B}\left(B_{s} \rightarrow \mu^{+} \mu^{-}\right)_{\mathrm{SM}} \\
& \mathcal{B}\left(B_{s} \rightarrow \mu^{+} \mu^{-}\right)_{\mathrm{SM}}=\tau_{B_{s}} \frac{G_{F}^{2}}{\pi}\left(\frac{\alpha}{4 \pi \sin ^{2} \theta_{W}}\right)^{2} F_{B_{s}}^{2} m_{\mu}^{2} m_{B_{s}} \sqrt{1-\frac{4 m_{\mu}^{2}}{m_{B_{s}}^{2}}}\left|V_{t b}^{*} V_{t s}\right|^{2} \eta_{\mathrm{eff}}^{2} Y_{0}\left(x_{t}\right)^{2}
\end{aligned}
$$




\begin{tabular}{|c||c||c|c|c|c|c|}
\hline Model & Scenario & $|P|$ & $\varphi_{P}$ & $|S|$ & $\varphi_{S}$ & $\varphi_{B_{s}}$ \\
\hline \hline CMFV & A & $|P|$ & 0 & 0 & 0 & 0 \\
MFV & D & $|P|$ & 0 & $|S|$ & 0 & 0 \\
LHT, 4G, RSc, $Z^{\prime}$ & $\mathrm{A}$ & $|P|$ & $\varphi_{P}$ & 0 & 0 & $\varphi_{B_{s}}$ \\
2HDM (Decoupling) & $\mathrm{C}$ & $|1 \mp S|$ & $\arg (1 \mp S)$ & $|S|$ & $\varphi_{S}$ & $\varphi_{B_{s}}$ \\
2HDM (A Dominance) & $\mathrm{A}$ & $|P|$ & $\varphi_{P}$ & 0 & 0 & $\varphi_{B_{s}}$ \\
2HDM (H Dominance) & $\mathrm{B}$ & 1 & 0 & $|S|$ & $\varphi_{S}$ & $\varphi_{B_{s}}$ \\
\hline
\end{tabular}

Table 6: General structure of basic variables in different NP models. The last three cases apply also to the MSSM. From [43].

with $\eta_{\text {eff }}$ and $Y_{0}\left(x_{t}\right)$ given below.

It follows that in any model the branching ratio without $\Delta \Gamma_{s}$ effect is related to the corresponding SM branching ratio through

$$
\mathcal{B}\left(B_{s} \rightarrow \mu^{+} \mu^{-}\right)=\mathcal{B}\left(B_{s} \rightarrow \mu^{+} \mu^{-}\right)_{\mathrm{SM}}\left(|P|^{2}+|S|^{2}\right),
$$

which is obtained from (115) by setting $y_{s}=0$.

Finally, all the formulae given above can be used for $B_{d} \rightarrow \mu^{+} \mu^{-}$with $s$ replaced by $d$ and $y_{d} \approx 0$ so that in this case there is no distinction between $\overline{\mathcal{B}}\left(B_{d} \rightarrow \mu^{+} \mu^{-}\right)$and $\mathcal{B}\left(B_{s} \rightarrow \mu^{+} \mu^{-}\right)$. Still the CP asymmetry $S_{\mu \mu}^{d}$ can be considered, although measuring it would be a heroic effort.

These formulae are very general and can be used to study these observables model independently using as variables

$$
|P|, \quad \varphi_{P}, \quad|S|, \quad \varphi_{S} .
$$

Such an analysis has been performed in [43]. The classification of popular NP in various scenarios characterized by the vanishing or non-vanishing values of the variables in (121) and of the new phase $\varphi_{B_{s}}$ in $B_{s}^{0}-\bar{B}_{s}^{0}$ mixing should help in monitoring the improved data in the future. While some of the results of this paper and also of related analysis of tree-level gauge boson and scalar contributions in [44] will be presented below, we collect already in Table 6 the properties of the selected models discussed in these two papers with respect to the basic phenomenological parameters listed in 121) and the classes defined in [43] they belong to.

After these general introduction we will discuss the results in the SM and its simplest extensions.

\subsubsection{Standard Model Results and the Data}

In the SM $B_{s, d} \rightarrow \mu^{+} \mu^{-}$are governed by $Z^{0}$-penguin diagrams and $\Delta F=1$ box diagrams which depend on the top-quark mass. The internal charm contribution can 
be safely neglected.

The only relevant Wilson coefficients in the $\mathrm{SM}$ are $C_{9}$ and $C_{10}$ given by

$$
\begin{aligned}
& \sin ^{2} \theta_{W} C_{9}^{\mathrm{SM}}=\sin ^{2} \theta_{W} P_{0}^{\mathrm{NDR}}+\left[\eta_{\mathrm{eff}} Y_{0}\left(x_{t}\right)-4 \sin ^{2} \theta_{W} Z_{0}\left(x_{t}\right)\right], \\
& \sin ^{2} \theta_{W} C_{10}^{\mathrm{SM}}=-\eta_{\mathrm{eff}} Y_{0}\left(x_{t}\right)
\end{aligned}
$$

with all the entries given in [40, 46] except for $\eta_{\text {eff }}$ which is discussed below. With $m_{s} \ll m_{b}$ we have $C_{9}^{\prime}=C_{10}^{\prime}=0$.

Here $Y_{0}\left(x_{t}\right)$ and $Z_{0}\left(x_{t}\right)$ are SM one-loop functions given by

$$
\begin{gathered}
Y_{0}\left(x_{t}\right)=\frac{x_{t}}{8}\left(\frac{x_{t}-4}{x_{t}-1}+\frac{3 x_{t} \log x_{t}}{\left(x_{t}-1\right)^{2}}\right) \\
Z_{0}(x)=-\frac{1}{9} \log x+\frac{18 x^{4}-163 x^{3}+259 x^{2}-108 x}{144(x-1)^{3}}+\frac{32 x^{4}-38 x^{3}-15 x^{2}+18 x}{72(x-1)^{4}} \log x .
\end{gathered}
$$

We have then

$$
C_{9}^{\mathrm{SM}} \approx 4.1, \quad C_{10}^{\mathrm{SM}} \approx-4.1 .
$$

The coefficient $\eta_{\text {eff }}$ was until recently denoted by $\eta_{Y}$ and included only NLO QCD corrections. For $m_{t}=m_{t}\left(m_{t}\right)$ one had $\eta_{Y}=1.012$ [151, 152].

Over several years electroweak corrections to the branching ratios have been calculated [153 156] but they were incomplete implying dependence on renormalization scheme used for electroweak parameters as analysed in detail in [157]. Recently complete NLO electroweak corrections [158] and QCD corrections up to NNLO [159] have been calculated. The inclusion of these new higher order corrections that were missing until now reduced significantly various scale uncertainties so that non-parametric uncertainties in both branching ratios are below $2 \%$.

The calculations performed in [158, 159] are very involved and in analogy to the QCD factors, like $\eta_{B}$ and $\eta_{1-3}$ in $\Delta F=2$ processes, we find it useful to include all QCD and electroweak corrections into $\eta_{\text {eff }}$ introduced in 1123 that without these corrections would be equal to unity. Inspecting the analytic formulae in [160] one finds then [47]

$$
\eta_{\text {eff }}=0.9882 \pm 0.0024 .
$$

The small departure of $\eta_{\text {eff }}$ from unity was already anticipated in [156, 157] but only the calculations in 158 160 could put these expectations and conjectures on firm footing. Indeed, in order to end up with such a simple result it was crucial to perform such involved calculations as these small corrections are only valid for particular definitions of the top-quark mass and of other electroweak parameters involved. In particular one has to use in $Y_{0}\left(x_{t}\right)$ the $\overline{\mathrm{MS}}$-renormalized top-quark mass $m_{t}\left(m_{t}\right)$ with respect to QCD 
but on-shell with respect to electroweak interactions. This means $m_{t}\left(m_{t}\right)=163.5 \mathrm{GeV}$ as calculated in [160]. Moreover, in using (127) to calculate observables like branching ratios it is important to have the same normalization of effective Hamiltonian as in the latter paper. There this normalization is expressed in terms of $G_{F}$ and $M_{W}$ only. Needless to say one can also use directly the formulae in [160].

In the present review we follow the normalization of effective Hamiltonian in [51] which uses $G_{F}, \alpha\left(M_{Z}\right)$ and $\sin ^{2} \theta_{W}$ and in order to be consistent with the calculation in 160] our $\eta_{\text {eff }}=0.991$ with $m_{t}\left(m_{t}\right)$ unchanged [47]. Interestingly also in the case of $K^{+} \rightarrow \pi^{+} \nu \bar{\nu}$ and $K_{L} \rightarrow \pi^{0} \nu \bar{\nu}$ the analog of $\eta_{\text {eff }}$, multiplying this time $X_{0}\left(x_{t}\right)$, is found with the normalizations of effective Hamiltonian in [51] and definition of $m_{t}$ as given above to be within $1 \%$ from unity [161]. It should be remarked that presently only in the case of the $B_{s, d} \rightarrow \mu^{+} \mu^{-}$decays discussed here and $K^{+} \rightarrow \pi^{+} \nu \bar{\nu}$ and $K_{L} \rightarrow \pi^{0} \nu \bar{\nu}$ decays considered in Step 8 one has to take such a care about the definition of $m_{t}$ with respect to electroweak corrections as in most cases such corrections are not known or hadronic uncertainties are too large so that the value $m_{t}\left(m_{t}\right)=163.0 \mathrm{GeV}$ in Table 1 used by us otherwise can easily be defended.

In view of still significant parametric uncertainties it is useful to show the dependence of the branching ratios on various input parameters involved. Such formulae have been already presented in $[43,157$ and have been recently updated by the authors of [158 and [159]. They find 160$]$

$$
\overline{\mathcal{B}}\left(B_{s} \rightarrow \mu^{+} \mu^{-}\right)_{\mathrm{SM}}=(3.65 \pm 0.06) \times 10^{-9}\left(\frac{m_{t}\left(m_{t}\right)}{163.5 \mathrm{GeV}}\right)^{3.02}\left(\frac{\alpha_{s}\left(M_{Z}\right)}{0.1184}\right)^{0.032} R_{s}
$$

where

$$
R_{s}=\left(\frac{F_{B_{s}}}{227.7 \mathrm{MeV}}\right)^{2}\left(\frac{\tau_{B_{s}}}{1.516 \mathrm{ps}}\right)\left(\frac{0.938}{r\left(y_{s}\right)}\right)\left|\frac{V_{t b}^{*} V_{t s}}{0.0415}\right|^{2},
$$

where precise definition of $m_{t}\left(m_{t}\right)$ is given below. We caution the reader that the parametric expression in (129), which is based on the results in [160], differs slightly from the one presented by these authors and consequently the quoted uncertainty is only an approximation but a very good one.

Proceeding in the same manner with $B_{d} \rightarrow \mu^{+} \mu^{-}$one finds 160

$$
\mathcal{B}\left(B_{d} \rightarrow \mu^{+} \mu^{-}\right)_{\mathrm{SM}}=(1.06 \pm 0.02) \times 10^{-10}\left(\frac{m_{t}\left(m_{t}\right)}{163.5 \mathrm{GeV}}\right)^{3.02}\left(\frac{\alpha_{s}\left(M_{Z}\right)}{0.1184}\right)^{0.032} R_{d}
$$

where

$$
R_{d}=\left(\frac{F_{B_{d}}}{190.5 \mathrm{MeV}}\right)^{2}\left(\frac{\tau_{B_{d}}}{1.519 \mathrm{ps}}\right)\left|\frac{V_{t b}^{*} V_{t d}}{0.0088}\right|^{2} .
$$

We emphasize that the overall factors in $(128)$ and 130 include all the corrections calculated in [158] and [159] and we do not expect that these numbers will change in the near future. On the other hand the central value of $\left|V_{t s}\right|$ in $(129)$ corresponds to 
the inclusive determination of $\left|V_{c b}\right| \approx 0.0425$. With $\left|V_{c b}\right| \approx 0.039$, as extracted from exclusive decays, one would find the central value for the branching ratio in question to be rather close to $3.0 \times 10^{-9}$.

Concerning the other two observables in (108), with $P=1$ and $S=0$ in the SM we have

$$
\mathcal{A}_{\Delta \Gamma}^{\mu \mu}=1, \quad S_{\mu \mu}^{s}=0, \quad r\left(y_{s}\right)=0.938 \pm 0.009 \quad(\mathrm{SM}) .
$$

Taking the parametric uncertainties into account one finds then 160

$$
\begin{array}{cl}
\overline{\mathcal{B}}\left(B_{s} \rightarrow \mu^{+} \mu^{-}\right)_{\mathrm{SM}}=(3.65 \pm 0.23) \times 10^{-9}, & \overline{\mathcal{B}}\left(B_{s} \rightarrow \mu^{+} \mu^{-}\right)_{\exp }=(2.9 \pm 0.7) \times 10^{-9}, \\
\mathcal{B}\left(B_{d} \rightarrow \mu^{+} \mu^{-}\right)_{\mathrm{SM}}=(1.06 \pm 0.09) \times 10^{-10}, & \mathcal{B}\left(B_{d} \rightarrow \mu^{+} \mu^{-}\right)_{\exp }=\left(3.6_{-1.4}^{+1.6}\right) \times 10^{-10}
\end{array}
$$

where we have also shown the most recent average of the results from LHCb and CMS 162 164. The agreement of the SM prediction with the data for $B_{s} \rightarrow \mu^{+} \mu^{-}$in 133 is remarkable, although the rather large experimental error still allows for sizable NP contributions. In $B_{d} \rightarrow \mu^{+} \mu^{-}$much bigger room for NP contributions is left.

We close our discussion of the SM with the correlations of $\mathcal{B}\left(B_{q} \rightarrow \mu^{+} \mu^{-}\right)$and $\Delta M_{s, d}$ that are free from $F_{B_{q}}$ and the $\left|V_{t q}\right|$ dependence [61]

$$
\begin{aligned}
& \mathcal{B}\left(B_{q} \rightarrow \mu^{+} \mu^{-}\right)=C \frac{\tau_{B_{q}}}{\hat{B}_{q}} \frac{\left(\eta_{\mathrm{eff}} Y_{0}\left(x_{t}\right)\right)^{2}}{S_{0}\left(x_{t}\right)} \Delta M_{q}, \\
& \text { with } \quad C=6 \pi \frac{1}{\eta_{B}^{2}}\left(\frac{\alpha}{4 \pi \sin ^{2} \theta_{W}}\right)^{2} \frac{m_{\mu}^{2}}{M_{W}^{2}}=4.291 \cdot 10^{-10},
\end{aligned}
$$

where $\hat{B}_{q}$, known from Step 2, enters linearly as opposed to quadratic dependence on $F_{B_{q}}$.

The results for branching ratios obtained in this manner have presently comparable errors to the ones obtained by direct calculations of branching ratios with their values close to the ones quoted above. Of interest are also the relations (13) and (14) with $r\left(\mu^{+} \mu^{-}\right)=1$ and $r=1$ which hopefully will be tested one day.

Let us next see what the simple models introduced in Section 3 can tell us about these decays.

\subsubsection{CMFV}

In this class of models there are no new $\mathrm{CP}$-violating phases and no new operators. Therefore all the formulae of the SM given until now remain valid except for the following changes: 
- The master functions $S_{0}\left(x_{t}\right)$ and $Y_{0}\left(x_{t}\right)$ are replaced by new functions $S(v)$ and $Y(v)$, respectively. Here $v$ denotes all parameters present in a given CMFV model, that is coupling and masses of new particles including those of the SM.

- QCD corrections to $B_{s, d} \rightarrow \mu^{+} \mu^{-}$, represented by $\eta_{Y}$, are expected in this class of models to be small and this is also expected for electroweak corrections. On the other hand $\eta_{B}$ could be visibly different in these models if the mases of particles involved are larger than $1 \mathrm{TeV}$. Yet, due to relatively small anomalous dimension of the $(V-A) \times(V-A)$ operator this change is much smaller than in the case of LR operators encountered in more complicated models. Therefore in view of new parameters present in $S(v)$, it is a good idea to use first just the SM value for $\eta_{B}$.

A more precise treatment would be to make the following replacement:

$$
S_{0}\left(x_{t}\right) \rightarrow S_{0}\left(x_{t}\right)+\frac{\eta_{B}^{\mathrm{NP}}}{\eta_{B}^{\mathrm{SM}}} \Delta S_{0}(v)
$$

where $\eta_{B}^{\mathrm{SM}}$ equals $\eta_{B}$ in previous expressions and $\Delta S_{0}(v)$ is the modification of the loop functions by NP contributions. The new $\eta_{B}^{\mathrm{NP}}$ can easily be calculated in the LO if the $\mathrm{NP}$ scale is known. Then the sign of the anomalous dimension of the operator $Q_{1}^{\mathrm{VLL}}$ implies $\eta_{B}^{\mathrm{NP}} \leq \eta_{B}^{\mathrm{SM}}$ for NP scales larger than the electroweak scale.

The branching ratios for $B_{s, d} \rightarrow \mu^{+} \mu^{-}$will now be modified with respect to the SM but as seen in Fig. 17 due to relations in (13) and (14) with $r\left(\mu^{+} \mu^{-}\right)=1$ and $r=1$ strong correlation between these two branching ratios is predicted. In Fig. 17 we included $\Delta \Gamma_{s}$ effects in $\mathcal{B}\left(B_{s} \rightarrow \mu^{+} \mu^{-}\right)$.

The calculations simplify considerably if CKM factors are fixed in Step 1. Then independently of $q$ we simply have

$$
\frac{\mathcal{B}\left(B_{q} \rightarrow \mu^{+} \mu^{-}\right)}{\mathcal{B}\left(B_{q} \rightarrow \mu^{+} \mu^{-}\right)^{\mathrm{SM}}}=\left(\frac{Y(v)}{Y_{0}\left(x_{t}\right)}\right)^{2}
$$

and consequently

$$
\left(\frac{\overline{\mathcal{B}}\left(B_{s} \rightarrow \mu^{+} \mu^{-}\right)}{\mathcal{B}\left(B_{d} \rightarrow \mu^{+} \mu^{-}\right)}\right)_{\mathrm{CMFV}}=\left(\frac{\overline{\mathcal{B}}\left(B_{s} \rightarrow \mu^{+} \mu^{-}\right)}{\mathcal{B}\left(B_{d} \rightarrow \mu^{+} \mu^{-}\right)}\right)_{\mathrm{SM}}=34.4 \pm 3.6,
$$

where we have used the SM values in (133) and (134). Using (14) with $r=1$ we would find $33.9 \pm 0.8$. Using (139) together with the measurement of $\overline{\mathcal{B}}\left(B_{s} \rightarrow \mu^{+} \mu^{-}\right)$(133) implies in turn in the context of these models

$$
\mathcal{B}\left(B_{d} \rightarrow \mu^{+} \mu^{-}\right)=(0.84 \pm 0.19) \times 10^{-10}, \quad(\mathrm{CMFV}),
$$

which is well be below the data in (134). This could then be an indication for new sources of flavour violation. In fact as seen in Fig. 17 the present data differ from CMFV correlation between these two branching ratios by roughly $2 \sigma$ but we have to wait for new improved data in order to claim NP at work. Still it will be interesting to see what kind of NP could bring the theory close to the present experimental central values for the branching ratios in this figure. 


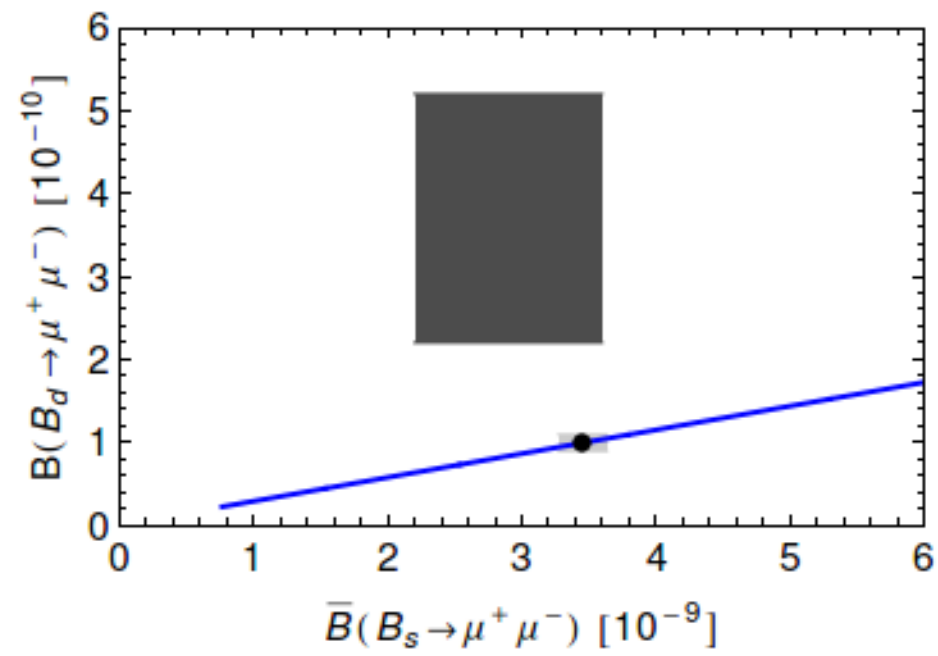

Figure 17: $\mathcal{B}\left(B_{d} \rightarrow \mu^{+} \mu^{-}\right)$vs $\overline{\mathcal{B}}\left(B_{s} \rightarrow \mu^{+} \mu^{-}\right)$in models with $C M F V$. SM is represented by the light grey area with black dot. Dark gray region: Combined exp $1 \sigma$ range $\overline{\mathcal{B}}\left(B_{s} \rightarrow\right.$ $\left.\mu^{+} \mu^{-}\right)=(2.9 \pm 0.7) \cdot 10^{-9}$ and $\mathcal{B}\left(B_{d} \rightarrow \mu^{+} \mu^{-}\right)=\left(3.6_{-1.4}^{+1.6}\right) \cdot 10^{-10}$.

\subsection{5 $2 \mathrm{HDM}_{\overline{\mathrm{MFV}}}$}

In $2 \mathrm{HDM}_{\overline{\mathrm{MFV}}}$ scalar and pseudoscalar penguin diagrams generate new scalar and pseudoscalar operators that can even dominate the decays $B_{s, d} \rightarrow \mu^{+} \mu^{-}$at sufficiently high value of $\tan \beta$. However, due to recent $\mathrm{LHCb}$ and CMS results such large enhancements are not possible for $B_{s} \rightarrow \mu^{+} \mu^{-}$anymore and within this model the same applies to $B_{d} \rightarrow \mu^{+} \mu^{-}$. Indeed within an excellent approximation we have then similarly to (139) 140

$$
\left(\frac{\mathcal{B}\left(B_{s} \rightarrow \mu^{+} \mu^{-}\right)}{\mathcal{B}\left(B_{d} \rightarrow \mu^{+} \mu^{-}\right)}\right)_{2 \mathrm{HDM}_{\overline{\mathrm{MFV}}}}=\left(\frac{\mathcal{B}\left(B_{s} \rightarrow \mu^{+} \mu^{-}\right)}{\mathcal{B}\left(B_{d} \rightarrow \mu^{+} \mu^{-}\right)}\right)_{\mathrm{SM}} .
$$

Combined with (85) we then conclude that also (14) with $r=1$ is well satisfied in this model. However, while the ratios in (85) and (141) are the same in $2 \mathrm{HDM}_{\overline{\mathrm{MFV}}}$ and the SM, the individual $\Delta M_{s, d}$ and $\mathcal{B}\left(B_{s, d} \rightarrow \mu^{+} \mu^{-}\right)$can differ in these models. Still the range for $\mathcal{B}\left(B_{d} \rightarrow \mu^{+} \mu^{-}\right)$in 140 also applies and constitutes an important test of this model.

Finally in the limit $C_{S}=-C_{P}$ lower bounds on the two branching ratios can be derived 43, 165):

$$
\mathcal{B}\left(B_{q} \rightarrow \mu^{+} \mu^{-}\right)_{2 \mathrm{HDM}_{\overline{\mathrm{MFV}}}} \geq \frac{1}{2}\left(1-y_{q}\right) \mathcal{B}\left(B_{q} \rightarrow \mu^{+} \mu^{-}\right)_{\mathrm{SM}},
$$

which are also valid in the MSSM 166. 


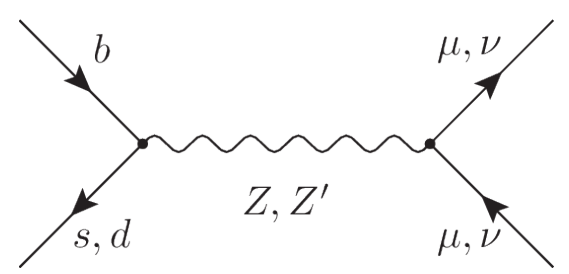

Figure 18: Tree-level flavour-changing $Z$ and $Z^{\prime}$ contribution to $\Delta F=1$ transitions.

\subsubsection{Tree-Level Gauge Boson Exchange}

We will next consider the contributions of a tree-level gauge boson exchange to the Wilson coefficients of the operators involved (see Fig. 18). Including the SM contributions one has $[40]$

$$
\begin{aligned}
& \sin ^{2} \theta_{W} C_{9}=\left[\eta_{Y} Y_{0}\left(x_{t}\right)-4 \sin ^{2} \theta_{W} Z_{0}\left(x_{t}\right)\right]-\frac{1}{g_{\mathrm{SM}}^{2}} \frac{1}{M_{Z^{\prime}}^{2}} \frac{\Delta_{L}^{s b}\left(Z^{\prime}\right) \Delta_{V}^{\mu \bar{\mu}}\left(Z^{\prime}\right)}{V_{t s}^{*} V_{t b}}, \\
& \sin ^{2} \theta_{W} C_{10}=-\eta_{Y} Y_{0}\left(x_{t}\right)-\frac{1}{g_{\mathrm{SM}}^{2}} \frac{1}{M_{Z^{\prime}}^{2}} \frac{\Delta_{L}^{s b}\left(Z^{\prime}\right) \Delta_{A}^{\mu \bar{\mu}}\left(Z^{\prime}\right)}{V_{t s}^{*} V_{t b}}, \\
& \sin ^{2} \theta_{W} C_{9}^{\prime}=-\frac{1}{g_{\mathrm{SM}}^{2}} \frac{1}{M_{Z^{\prime}}^{2}} \frac{\Delta_{R}^{s b}\left(Z^{\prime}\right) \Delta_{V}^{\mu \bar{\mu}}\left(Z^{\prime}\right)}{V_{t s}^{*} V_{t b}} \\
& \sin ^{2} \theta_{W} C_{10}^{\prime}=-\frac{1}{g_{\mathrm{SM}}^{2}} \frac{1}{M_{Z^{\prime}}^{2}} \frac{\Delta_{R}^{s b}\left(Z^{\prime}\right) \Delta_{A}^{\mu \bar{\mu}}\left(Z^{\prime}\right)}{V_{t s}^{*} V_{t b}}
\end{aligned}
$$

where we have defined

$$
\begin{aligned}
& \Delta_{V}^{\mu \bar{\mu}}\left(Z^{\prime}\right)=\Delta_{R}^{\mu \bar{\mu}}\left(Z^{\prime}\right)+\Delta_{L}^{\mu \bar{\mu}}\left(Z^{\prime}\right), \\
& \Delta_{A}^{\mu \bar{\mu}}\left(Z^{\prime}\right)=\Delta_{R}^{\mu \bar{\mu}}\left(Z^{\prime}\right)-\Delta_{L}^{\mu \bar{\mu}}\left(Z^{\prime}\right) .
\end{aligned}
$$

In order to simplify the presentation we still work with $\eta_{Y}$ and $Y_{0}\left(x_{t}\right)$ which should be replaced by $Y_{\text {eff }}$ in 123 if the future precision of experimental data will require it.

The vector Wilson coefficients $C_{9}, C_{9}^{\prime}$ do not contribute to decays in question but they will enter Step 7, where the decays $B \rightarrow X_{s} \ell^{+} \ell^{-}$and $B \rightarrow K^{*}(K) \ell^{+} \ell^{-}$are considered. Assuming that the CKM parameters have been determined independently of NP and are universal we find then

$$
\frac{\mathcal{B}\left(B_{q} \rightarrow \mu^{+} \mu^{-}\right)}{\mathcal{B}\left(B_{q} \rightarrow \mu^{+} \mu^{-}\right)^{\mathrm{SM}}}=\left|\frac{Y_{A}^{q}(v)}{\eta_{Y} Y_{0}\left(x_{t}\right)}\right|^{2},
$$

where

$$
Y_{A}^{q}(v)=\eta_{Y} Y_{0}\left(x_{t}\right)-\frac{1}{V_{t b} V_{t q}^{*}} \frac{\left[\Delta_{A}^{\mu \bar{\mu}}\left(Z^{\prime}\right)\right]}{M_{Z^{\prime}}^{2} g_{\mathrm{SM}}^{2}}\left[\Delta_{R}^{q b}\left(Z^{\prime}\right)-\Delta_{L}^{q b}\left(Z^{\prime}\right)\right]
$$




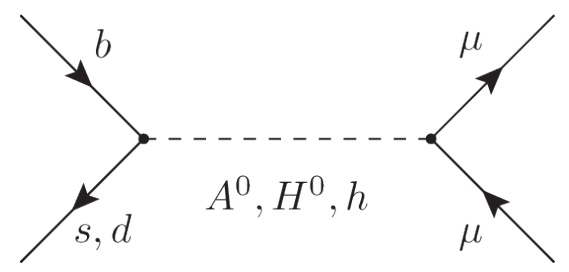

Figure 19: Tree-level flavour-changing $A^{0}, H^{0}, h$ contribution to $\Delta F=1$ transitions.

is generally complex and moreover different for $B_{d} \rightarrow \mu^{+} \mu^{-}$and $B_{s} \rightarrow \mu^{+} \mu^{-}$implying violation of the CMFV correlation shown in Fig. 17. Still the correlation between $\mathcal{B}\left(B_{q} \rightarrow \mu^{+} \mu^{-}\right)^{\mathrm{SM}}$ and $\Delta M_{q}$, when all these observables are calculated directly, could offer a useful test of the model.

In [40] the correlations between the following observables have been investigated:

$$
\Delta M_{s}, \quad S_{\psi \phi}, \quad \mathcal{B}\left(B_{s} \rightarrow \mu^{+} \mu^{-}\right), \quad S_{\mu \mu}^{s}
$$

in the $B_{s}$-system and

$$
\Delta M_{d}, \quad S_{\psi K_{S}}, \quad \mathcal{B}\left(B_{d} \rightarrow \mu^{+} \mu^{-}\right), \quad S_{\mu \mu}^{d}
$$

in $B_{d}$ system. To this end

$$
\Delta_{A}^{\mu \bar{\mu}}\left(Z^{\prime}\right)=0.5
$$

has been chosen, to be compared with its SM value $\Delta_{A}^{\mu \bar{\mu}}(Z)=0.372$.

Note that for fixed $\Delta_{A}^{\mu \bar{\mu}}\left(Z^{\prime}\right)$ the observables in 150 depend only on two complex variables $\Delta_{L, R}^{b s}\left(Z^{\prime}\right)$ and in fact in the LHS, RHS, LRS and ALR scenarios only on $\tilde{s}_{23}$ and $\delta_{23}$. Similarly the observables in (151) depend on only two complex variables $\Delta_{L, R}^{b d}\left(Z^{\prime}\right)$ and in the LHS, RHS, LRS and ALR scenarios only on $\tilde{s}_{13}$ and $\delta_{13}$. As these parameters have been already constrained in Step 3, definite correlations between the observables within each set in (150) and (151) follow. Once the $U(2)^{3}$ symmetry is imposed correlations between the sets in (150) and (151) are found. It will be interesting to investigate the impact on these correlations from $b \rightarrow s \ell^{+} \ell^{-}$and $b \rightarrow s \nu \bar{\nu}$ transitions that we consider in Steps 7 and 9, respectively.

It will be useful to present numerical analysis of these correlations together with the ones resulting from tree-level scalar exchanges and we will first turn our attention to the latter exchanges.

\subsubsection{Tree-Level Scalar and Pseudoscalar Exchanges}

A very detailed analysis of tree-level scalar and pseudoscalar tree-level contributions as shown in Fig. 19 to decays in question has been performed in [44]. In this case 
SM Wilson coefficients remain unchanged but the Wilson coefficients of scalar and pseudoscalar operators become non-zero and are given at $\mu=M_{H}$ as follows

$$
\begin{aligned}
& m_{b}\left(M_{H}\right) \sin ^{2} \theta_{W} C_{S}=\frac{1}{g_{\mathrm{SM}}^{2}} \frac{1}{M_{H}^{2}} \frac{\Delta_{R}^{s b}(H) \Delta_{S}^{\mu \bar{\mu}}(H)}{V_{t s}^{*} V_{t b}}, \\
& m_{b}\left(M_{H}\right) \sin ^{2} \theta_{W} C_{S}^{\prime}=\frac{1}{g_{\mathrm{SM}}^{2}} \frac{1}{M_{H}^{2}} \frac{\Delta_{L}^{s b}(H) \Delta_{S}^{\mu \bar{\mu}}(H)}{V_{t s}^{*} V_{t b}}, \\
& m_{b}\left(M_{H}\right) \sin ^{2} \theta_{W} C_{P}=\frac{1}{g_{\mathrm{SM}}^{2}} \frac{1}{M_{H}^{2}} \frac{\Delta_{R}^{s b}(H) \Delta_{P}^{\mu \bar{\mu}}(H)}{V_{t s}^{*} V_{t b}}, \\
& m_{b}\left(M_{H}\right) \sin ^{2} \theta_{W} C_{P}^{\prime}=\frac{1}{g_{\mathrm{SM}}^{2}} \frac{1}{M_{H}^{2}} \frac{\Delta_{L}^{s b}(H) \Delta_{P}^{\mu \bar{\mu}}(H)}{V_{t s}^{*} V_{t b}},
\end{aligned}
$$

where

$$
\begin{aligned}
& \Delta_{S}^{\mu \bar{\mu}}(H)=\Delta_{R}^{\mu \bar{\mu}}(H)+\Delta_{L}^{\mu \bar{\mu}}(H), \\
& \Delta_{P}^{\mu \bar{\mu}}(H)=\Delta_{R}^{\mu \bar{\mu}}(H)-\Delta_{L}^{\mu \bar{\mu}}(H) .
\end{aligned}
$$

Here $H$ stands for a scalar or pseudoscalar but if the mass eigenstates has a given CP-parity it is useful to distinguish between a scalar $\left(H^{0}\right)$ and a pseudoscalar $\left(A^{0}\right)$. Then

$$
\Delta_{S}^{\mu \bar{\mu}}\left(A^{0}\right)=0, \quad \Delta_{P}^{\mu \bar{\mu}}\left(H^{0}\right)=0
$$

and only $\Delta_{S}^{\mu \bar{\mu}}\left(H^{0}\right)$ and $\Delta_{P}^{\mu \bar{\mu}}\left(A^{0}\right)$ can be non-vanishing. This is not a general property and in fact in the presence of CP-violating effects scalar and pseudoscalars can have both couplings. For simplicity, as in [44], we will assume (158) to be true.

The crucial property of these couplings following from the hermicity of the Hamiltonian is that $\Delta_{S}^{\mu \bar{\mu}}$ is real and $\Delta_{P}^{\mu \bar{\mu}}$ purely imaginary. Therefore it is useful to work with

$$
\Delta_{P}^{\mu \bar{\mu}}\left(A^{0}\right)=i \tilde{\Delta}_{P}^{\mu \bar{\mu}}\left(A^{0}\right)
$$

where $\tilde{\Delta}_{P}^{\mu \bar{\mu}}\left(A^{0}\right)$ is real.

It should be emphasized that in terms of the couplings used in the analysis of $B_{s, d}^{0}-\bar{B}_{s, d}^{0}$ mixings we have generally

$$
\Delta_{R}^{s b}(H)=\left[\Delta_{L}^{b s}(H)\right]^{*}, \quad \Delta_{L}^{s b}(H)=\left[\Delta_{R}^{b s}(H)\right]^{*},
$$

which should be kept in mind when studying correlations between $\Delta F=1$ and $\Delta F=2$ transitions.

Concerning the values of the $\tilde{\Delta}_{P}^{\mu \bar{\mu}}(H)$ and $\Delta_{S}^{\mu \bar{\mu}}(H)$ we will set as in 44

$$
\tilde{\Delta}_{P}^{\mu \bar{\mu}}(H)= \pm 0.020 \frac{m_{b}\left(M_{H}\right)}{m_{b}\left(m_{b}\right)}, \quad \Delta_{S}^{\mu \bar{\mu}}(H)=0.040 \frac{m_{b}\left(M_{H}\right)}{m_{b}\left(m_{b}\right)}
$$



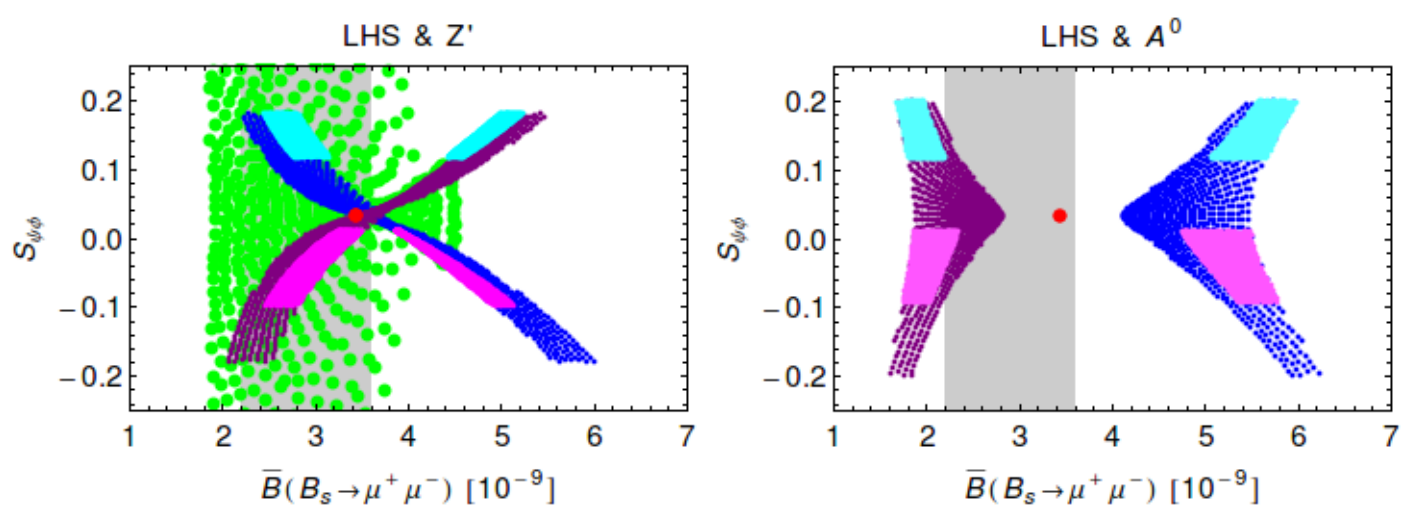

Figure 20: $S_{\psi \phi}$ versus $\overline{\mathcal{B}}\left(B_{s} \rightarrow \mu^{+} \mu^{-}\right)$for $Z^{\prime}$ exchange with $M_{Z^{\prime}}=1 \mathrm{TeV}$ (left) and $A^{0}$ case with $M_{A^{0}}=1 \mathrm{TeV}$ (right) in LHS for two oases. The blue and purple regions are almost identical for LHS1 and LHS2. The magenta region corresponds to the $U(2)^{3}$ limit for LHS1 and the cyan region for LHS2. The green points in the $Z^{\prime}$ case indicate the regions that are compatible with $b \rightarrow s \ell^{+} \ell^{-}$constraints of [38]. In the $A^{0}$ case $b \rightarrow s \ell^{+} \ell^{-}$does not give additional constraints. Gray region: exp $1 \sigma$ range $\overline{\mathcal{B}}\left(B_{s} \rightarrow\right.$ $\left.\mu^{+} \mu^{-}\right)=(2.9 \pm 0.7) \cdot 10^{-9}$. Red point: SM central value.

with the latter factor being 0.61 for $M_{H}=1 \mathrm{TeV}$. We show this factor explicitly to indicate how the correct scale for $m_{b}$ affects the allowed range for the lepton couplings. These values assure significant NP effects in $B_{s, d} \rightarrow \mu^{+} \mu^{-}$while being consistent will all known data.

\subsubsection{Comparison of tree-level $Z^{\prime}$, pseudoscalar and scalar exchanges}

In Fig. 20 we show the correlation between $\overline{\mathcal{B}}\left(B_{s} \rightarrow \mu^{+} \mu^{-}\right.$) and $S_{\psi \phi}$ for $Z^{\prime}$ (left panel) and $A^{0}$ (right panel). The corresponding plots for the correlation between $S_{\mu \mu}^{s}$ and $S_{\psi \phi}$ and $\mathcal{A}_{\Delta \Gamma}^{\mu \mu}$ and $S_{\psi \phi}$ are shown in Figs. 21 and 22 . In Fig. 23 we show the corresponding results for the scalar $H^{0}$.

The colour coding is as follows:

- In the general case blue and purple allowed regions correspond to oases with small and large $\delta_{23}$, respectively.

- In the $U(2)^{3}$ symmetry case, the allowed region are shown in magenta and cyan for LHS1 and LHS2, respectively, as in this case even in the $B_{s}$ system there is dependence on $\left|V_{u b}\right|$ scenario. These regions are subregions of the general blue or purple regions so that they cover some parts of them.

- The green points in the $Z^{\prime}$ case indicate the region that is compatible with constraints from $b \rightarrow s \ell^{+} \ell^{-}$transitions. In the scalar and pseudoscalar case the whole oases are compatible with $b \rightarrow s \ell^{+} \ell^{-}$(see also Sec. 5.7.3). 

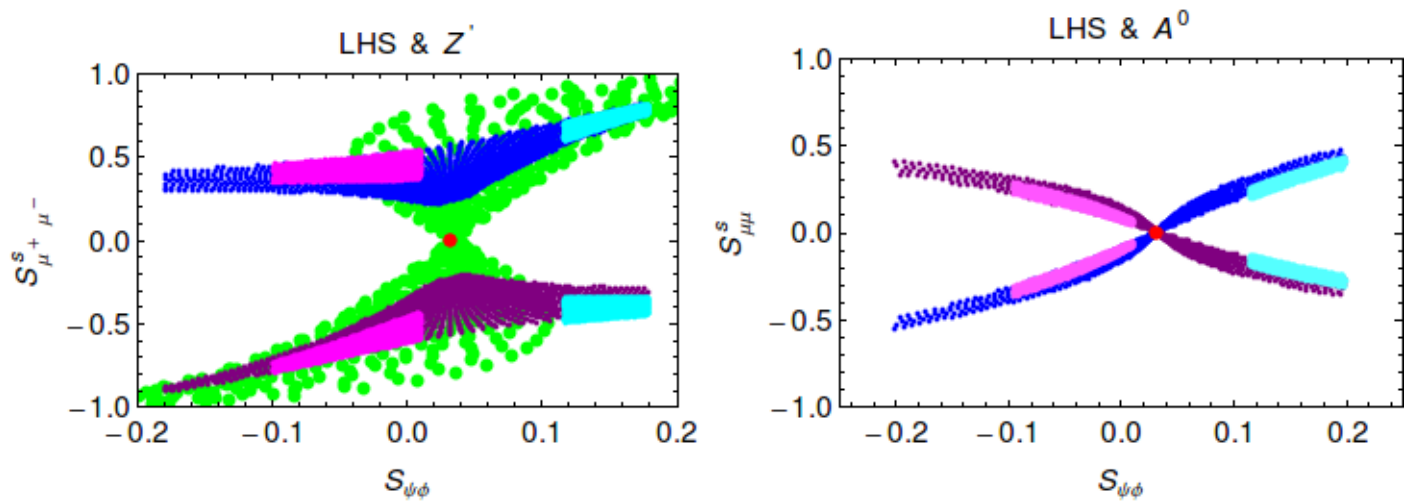

Figure 21: $S_{\mu^{+} \mu^{-}}^{s}$ versus $S_{\psi \phi}$ in LHS1 and for $Z^{\prime}$ (left) and pseudoscalar $A^{0}$ case (right) both for $1 \mathrm{TeV}$. The magenta region corresponds to the $U(2)^{3}$ limit for LHS1 and the cyan region for LHS2. The green points in the $Z^{\prime}$ case indicate the regions that are compatible with $b \rightarrow s \ell^{+} \ell^{-}$constraints. In the $A^{0}$ case $b \rightarrow s \ell^{+} \ell^{-}$does not give additional constraints. Red point: SM central value.
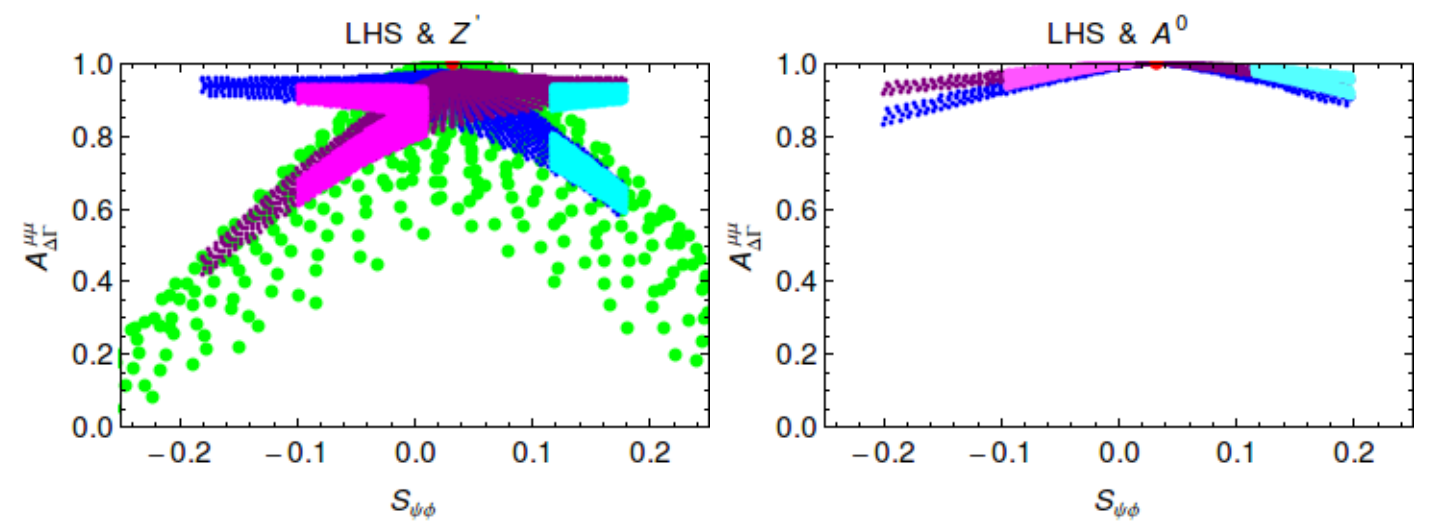

Figure 22: $\mathcal{A}_{\Delta \Gamma}^{\lambda}$ versus $S_{\psi \phi}$ in LHS1 and for $Z^{\prime}$ (left) and pseudoscalar $A^{0}$ case (right) both for $1 \mathrm{TeV}$. The magenta region corresponds to the $U(2)^{3}$ limit for LHS1 and the cyan region for LHS2. The green points in the $Z^{\prime}$ case indicate the regions that are compatible with $b \rightarrow s \ell^{+} \ell^{-}$constraints. In the $A^{0}$ case $b \rightarrow s \ell^{+} \ell^{-}$does not give additional constraints. Red point: SM central value.

We observe several striking differences between the results for $Z^{\prime}, A^{0}$ and $H^{0}$ which allow to distinguish these scenarios from each other:

- In the $A^{0}$ case the asymmetry $S_{\mu^{+} \mu^{-}}^{s}$ can be zero while this is not the case for $Z^{\prime}$ where the requirement of suppression of $\Delta M_{s}$ directly translates in $S_{\mu^{+} \mu^{-}}^{s}$ being non-zero. Consequently in the $Z^{\prime}$ case the sign of $S_{\mu^{+} \mu^{-}}^{s}$ can be used to identify the right oasis. This is not possible in the case of $A^{0}$.

- On the other hand we observe that in the $A^{0}$ case the measurement of $\overline{\mathcal{B}}\left(B_{s} \rightarrow\right.$ 

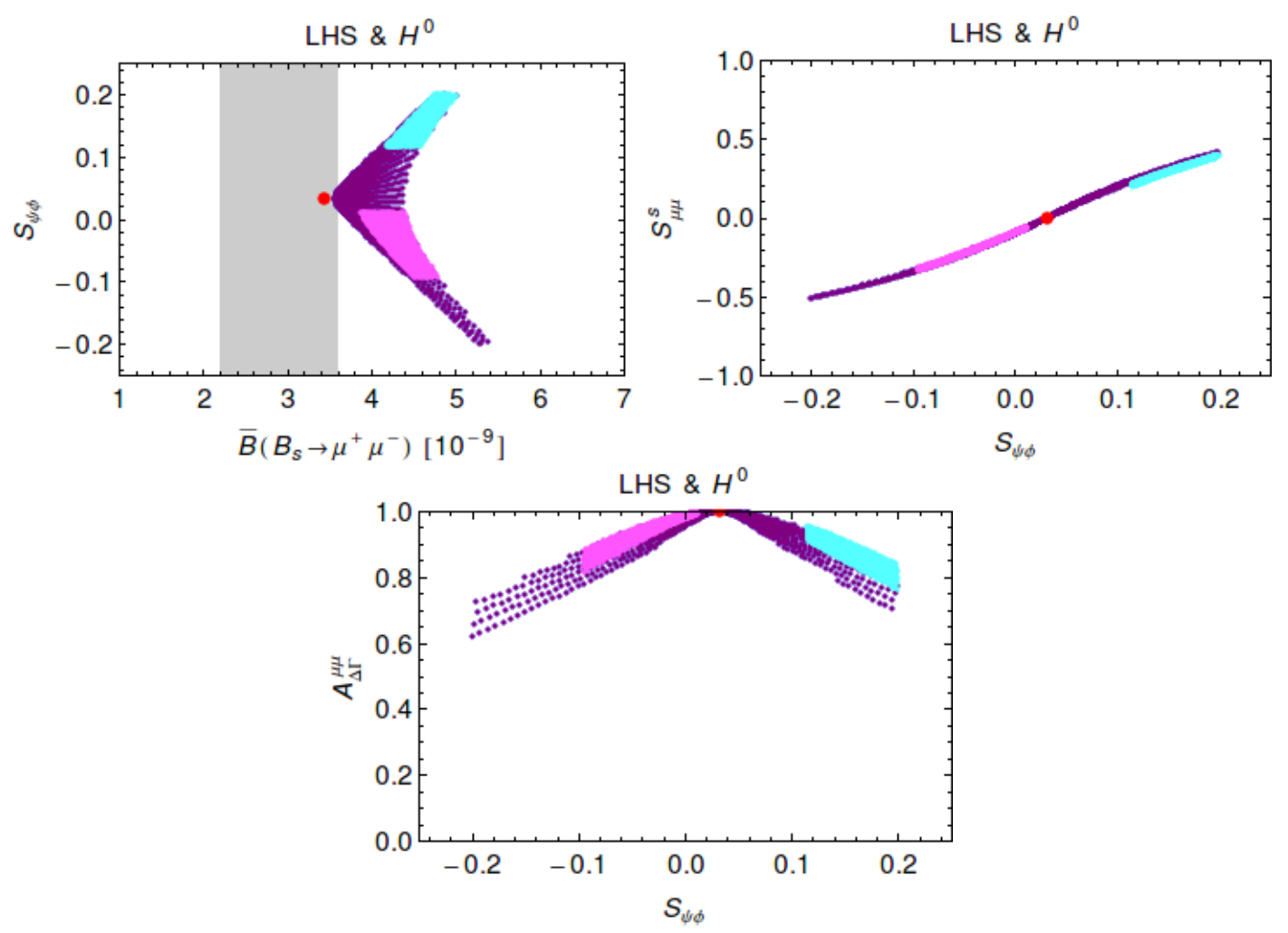

Figure 23: $S_{\psi \phi}$ versus $\overline{\mathcal{B}}\left(B_{s} \rightarrow \mu^{+} \mu^{-}\right), S_{\mu^{+} \mu^{-}}^{s}$ versus $S_{\psi \phi}$ and $\mathcal{A}_{\Delta \Gamma}^{\lambda}$ versus $S_{\psi \phi}$ for scalar $H^{0}$ case with $M_{H}=1 \mathrm{TeV}$ in LHS1. The two oases (blue and purple) overlap. The magenta region corresponds to the $U(2)^{3}$ limit for LHS1 and the cyan region for LHS2. Red point: SM central value.

$\mu^{+} \mu^{-}$) uniquely chooses the right oasis. The enhancement of this branching ratio relatively to the SM chooses the blue oasis while suppression the purple one. Present data from LHCb and CMS favour the purple oasis. This distinction is not possible in the $Z^{\prime}$ case. The maximal enhancements and suppressions are comparable in both cases but finding $\overline{\mathcal{B}}\left(B_{s} \rightarrow \mu^{+} \mu^{-}\right)$close to SM value would require in the $A^{0}$ case either larger $M_{H}$ or smaller muon coupling.

- Concerning the $H^{0}$ case, the absence of the interference with the SM contribution implies that $\overline{\mathcal{B}}\left(B_{s} \rightarrow \mu^{+} \mu^{-}\right)$can only be enhanced in this scenario and this result is independent of the oasis considered. Thus finding this branching ratio below its SM value would favour the other two scenarios over scalar one. The present data from $\mathrm{LHCb}$ and $\mathrm{CMS}$ indicate that indeed this could be the case. But the enhancement is not as pronounced as in the pseudoscalar case because in the absence of the interference with the SM contribution the correction to the branching ratio is governed here by the square of the muon coupling and is not linearly proportional to it as in the pseudoscalar case. Therefore in order to 

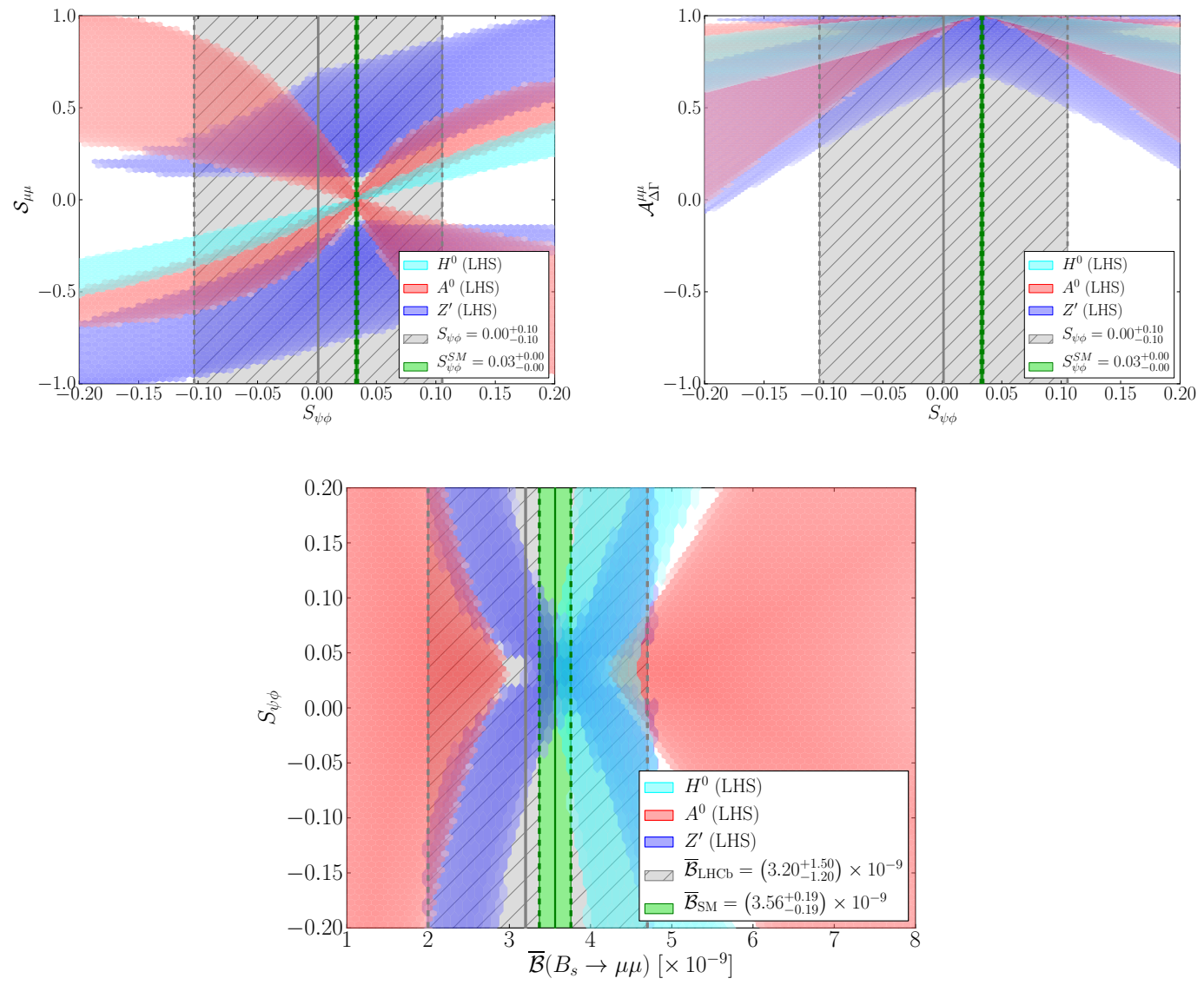

Figure 24: Overlay of the correlations for $S_{\mu \mu}^{s}$ versus $S_{\psi \phi}$ (top left), $A_{\Delta \Gamma}^{\mu \mu}$ versus $S_{\psi \phi}$ (top right) and $S_{\psi \phi}$ versus $\overline{\mathcal{B}}\left(B_{s} \rightarrow \mu^{+} \mu^{-}\right.$) (bottom) for tree level scalar (cyan), pseudoscalar (red) and $Z^{\prime}$ (blue) exchange (both oases in same colour respectively) in LHS. The lepton couplings are varied in the ranges $\left|\Delta_{S, P}^{\mu \mu}(H)\right| \in[0.012,0.024]$ and $\Delta_{A}^{\mu \mu}\left(Z^{\prime}\right) \in[0.3,0.7]$. From [44].

exclude this scenario requires significant reduction of experimental errors.

- Also CP-asymmetries in the $H^{0}$ case differ from $Z^{\prime}$ and $A^{0}$ cases. Similarly to the branching ratio there is no dependence on the oasis considered but more importantly $S_{\mu^{+} \mu^{-}}^{S}$ can only increase with increasing $S_{\psi \phi}$.

- The correlation between $\mathcal{A}_{\Delta \Gamma}^{\lambda}$ and $S_{\psi \phi}$ has the same structure for $Z^{\prime}, A^{0}$ and $H^{0}$ cases. We observe that for $M_{H}=1 \mathrm{TeV}$, even for $S_{\psi \phi}$ significantly different from zero, $\mathcal{A}_{\Delta \Gamma}^{\lambda}$ does not differ significantly from unity in $A^{0}$ and $H^{0}$ scenarios. Larger effects for the same mass are found in the $Z^{\prime}$ case.

In Fig. 24 we summarize our results in the $B_{s}$ system for tree level $Z^{\prime}, H^{0}$ and $A^{0}$ 
exchanges where we also vary the lepton couplings in a wider range: $\left|\Delta_{S, P}^{\mu \mu}(H)\right| \in$ $[0.012,0.024]$ and $\Delta_{A}^{\mu \mu}\left(Z^{\prime}\right) \in[0.3,0.7]$. As explained in [44 the striking differences between the $A^{0}$-scenario and $Z^{\prime}$-scenario can be traced back to the difference between the phase of the NP correction to the quantity $P$, defined in $(113)$, in these two NP scenarios. As the oasis structure as far as the phase $\delta_{23}$ is concerned is the same in both scenarios the difference enters through the muon couplings which are imaginary in the case of $A^{0}$-scenario but real in the case of $Z^{\prime}$. Taking in addition into account the sign difference between $Z^{\prime}$ and pseudoscalar propagator in the the $b \rightarrow s \mu^{+} \mu^{-}$amplitude, which is now not compensated by a hadronic matrix element, one finds that

$$
P\left(Z^{\prime}\right)=1+r_{Z^{\prime}} e^{i \delta_{Z^{\prime}}}, \quad P\left(A^{0}\right)=1+r_{A^{0}} e^{i \delta_{A^{0}}}
$$

with

$$
r_{Z^{\prime}} \approx r_{A^{0}}, \quad \delta_{Z^{\prime}}=\delta_{23}-\beta_{s}, \quad \delta_{A^{0}}=\delta_{Z^{\prime}}-\frac{\pi}{2} .
$$

Therefore with $\delta_{23}$ of Fig. 10 the phase $\delta_{Z^{\prime}}$ is around $90^{\circ}$ and $270^{\circ}$ for the blue and purple oasis, respectively. Correspondingly $\delta_{A^{0}}$ is around $0^{\circ}$ and $180^{\circ}$. This difference in the phases is at the origin of the differences listed above. In particular, we understand now why the CP asymmetry $S_{\mu^{+} \mu^{-}}^{s}$ can vanish in the $A^{0}$ case, while it was always different from zero in the $Z^{\prime}$-case. What is interesting is that this difference is just related to the different particle exchanged: gauge boson and pseudoscalar. We summarize the ranges of $\delta_{Z^{\prime}}$ and $\delta_{A^{0}}$ in Table 7 .

Proceeding in an analogous manner for the scalar case we arrive at an important relation:

$$
\varphi_{S}=\delta_{Z^{\prime}}-\pi
$$

where the shift is related to the sign difference in the $Z^{\prime}$ and scalar propagators. But as seen in (115)-(117) the three observables given there, all depend on $2 \varphi_{S}$, implying that from the point of view of these quantities this shift is irrelevant. As different oases correspond to phases shifted by $\pi$ this also explains why in the scalar case the results in different oases are the same. That the branching ratio can only be enhanced follows just from the absence of the interference with the SM contributions. In order to understand the signs in $S_{\mu \mu}^{s}$ one should note the minus sign in front of sine in the corresponding formula. Rest follows from 164 and Table 7.

We now turn our attention to the $B_{d} \rightarrow \mu^{+} \mu^{-}$decay. Here we have to distinguish between LHS1 and LHS2 scenarios. Our colour coding is such that in the general case yellow and green allowed regions correspond to oases with small and large $\delta_{13}$, respectively. We do not show the impact of the imposition of the $U(2)^{3}$ symmetry as the resulting reduction of the allowed areas amounts typically to $5-10 \%$ at most and it is more transparent not to show it.

In Figs. 25 and 26 we show $S_{\psi K_{S}}$ vs $\mathcal{B}\left(B_{d} \rightarrow \mu^{+} \mu^{-}\right)$and $S_{\mu \mu}^{d}$ vs $\mathcal{B}\left(B_{d} \rightarrow \mu^{+} \mu^{-}\right)$for $Z^{\prime}$ scenario. The corresponding plots for the $A^{0}$ and $H^{0}$ scenarios are shown in Figs. 27, 30. 


\begin{tabular}{|c||c|c|}
\hline Oasis & $\delta_{Z^{\prime}}$ & $\delta_{A^{0}}$ \\
\hline \hline$B_{s}$ (blue) & $50^{\circ}-130^{\circ}$ & $-40^{\circ}-\left(+40^{\circ}\right)$ \\
$B_{s}$ (purple) & $230^{\circ}-310^{\circ}$ & $140^{\circ}-220^{\circ}$ \\
\hline$B_{d}(\mathrm{~S} 1)$ (yellow) & $57^{\circ}-86^{\circ}$ & $-33^{\circ}-\left(+4^{\circ}\right)$ \\
$B_{d}$ (S1) (green) & $237^{\circ}-266^{\circ}$ & $147^{\circ}-176^{\circ}$ \\
$B_{d}(\mathrm{~S} 2)$ (yellow) & $103^{\circ}-125^{\circ}$ & $13^{\circ}-35^{\circ}$ \\
$B_{d}$ (S2) (green) & $283^{\circ}-305^{\circ}$ & $193^{\circ}-215^{\circ}$ \\
\hline$U(2)^{3}(\mathrm{~S} 1)$ (blue, magenta) & $55^{\circ}-84^{\circ}$ & $-35^{\circ}-\left(-6^{\circ}\right)$ \\
$U(2)^{3}(\mathrm{~S} 1)$ (purple, magenta) & $235^{\circ}-264^{\circ}$ & $145^{\circ}-174^{\circ}$ \\
$U(2)^{3}$ (S2) (blue, cyan) & $101^{\circ}-121^{\circ}$ & $11^{\circ}-31^{\circ}$ \\
$U(2)^{3}$ (S2) (purple, cyan) & $291^{\circ}-301^{\circ}$ & $201^{\circ}-211^{\circ}$ \\
\hline
\end{tabular}

Table 7: Ranges for the values of $\delta_{Z^{\prime}}$ and $\delta_{A^{0}}$ as defined in (162) for the $B_{s}$ and $B_{d}$ systems and various cases discussed in the text. Also the result for $U(2)^{3}$ models is shown. From [44].
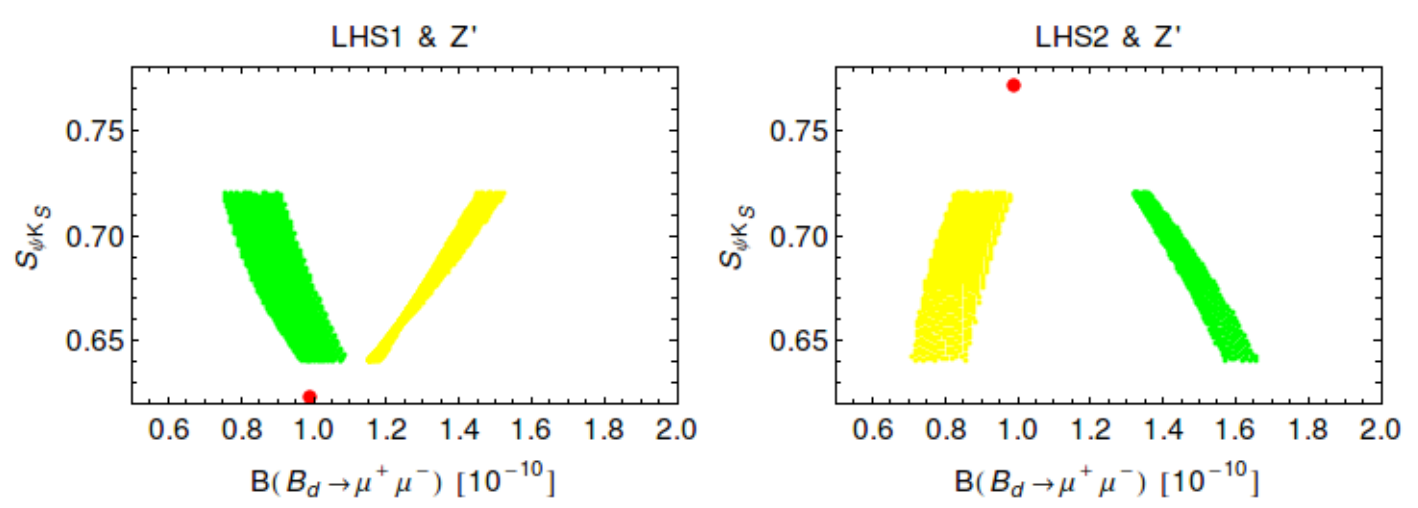

Figure 25: $S_{\psi K_{S}}$ versus $\mathcal{B}\left(B_{d} \rightarrow \mu^{+} \mu^{-}\right)$for $M_{Z^{\prime}}=1 \mathrm{TeV}$ in LHS1 (left) and LHS2 (right) for the yellow and green oases. Red point: SM central value.

In order to understand the differences between these two scenarios of NP we again look at the phase of the correction to $P$ in $(162)$ which now is given as follows:

$$
r_{Z^{\prime}} \approx r_{A^{0}}, \quad \delta_{Z^{\prime}}=\delta_{13}-\beta, \quad \delta_{A^{0}}=\delta_{Z^{\prime}}-\frac{\pi}{2} .
$$

Note that this time the phase of $V_{t d}$ enters the analysis with $\beta \approx 19^{\circ}$ and $\beta \approx 25^{\circ}$ for S1 and S2 scenario of $\left|V_{u b}\right|$, respectively. We find then that in scenario S2 the phase $\delta_{Z^{\prime}}$ is around $115^{\circ}$ and $295^{\circ}$ for yellow and green oases, respectively. Correspondingly $\delta_{A^{0}}$ is around $25^{\circ}$ and $205^{\circ}$. We summarize the ranges of $\delta_{Z^{\prime}}$ and $\delta_{A^{0}}$ in Table 7 . 

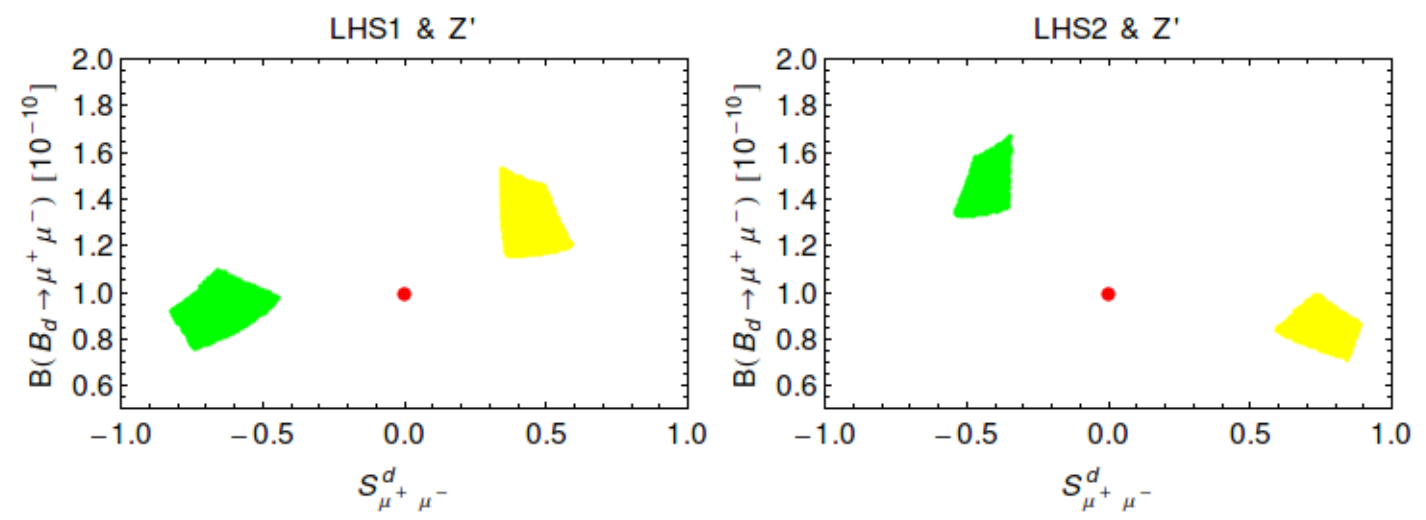

Figure 26: $\mathcal{B}\left(B_{d} \rightarrow \mu \bar{\mu}\right)$ versus $S_{\mu^{+} \mu^{-}}^{d}$ for $M_{Z^{\prime}}=1 \mathrm{TeV}$ in LHS1 (left) and LHS2 (right). Red point: $S M$ central value.
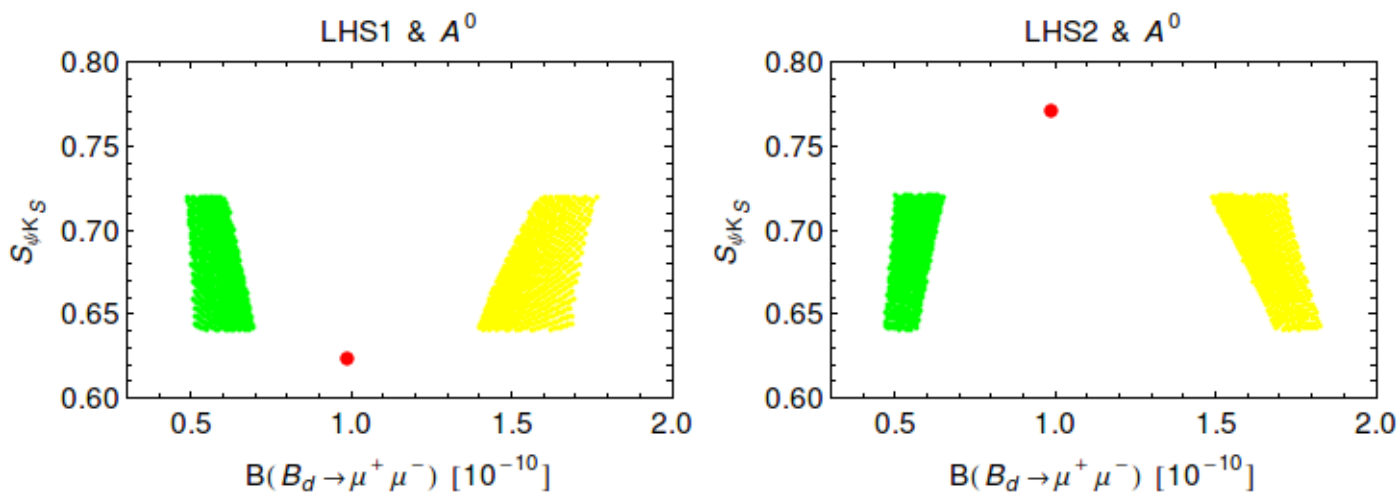

Figure 27: $S_{\psi K_{S}}$ versus $\mathcal{B}\left(B_{d} \rightarrow \mu^{+} \mu^{-}\right)$in $A^{0}$ scenario for $M_{H}=1$ TeV in LHS1 (left) and LHS2 (right) in the yellow and green oases as discussed in the text. Red point: SM central value.

With this insight at hand we can easily understand the plots in question noting that the enhancements and suppressions of $\mathcal{B}\left(B_{d} \rightarrow \mu^{+} \mu^{-}\right)$are governed by the cosine of the phase and the signs of $S_{\mu \mu}^{d}$ by the corresponding sines. We leave this exercise to the motivated readers and refer to 44 for a detailed description of the plots. What is interesting is that already the suppressions or enhancements of certain observables and correlations or anti-correlations between them could tell us one day which of the three $\mathrm{NP}$ scenarios if any is favoured by nature. In fact if the present central experimental value for $\mathcal{B}\left(B_{d} \rightarrow \mu^{+} \mu^{-}\right)$will be confirmed by more precise measurements tree-level $Z^{\prime}$, $A^{0}$ and $H^{0}$ exchanges will not be able to describe such data alone when the constraints from $\Delta F=2$ transitions are taken into account.

Finally, let us make a few comments on the impact of the imposition of the $U(2)^{3}$ 

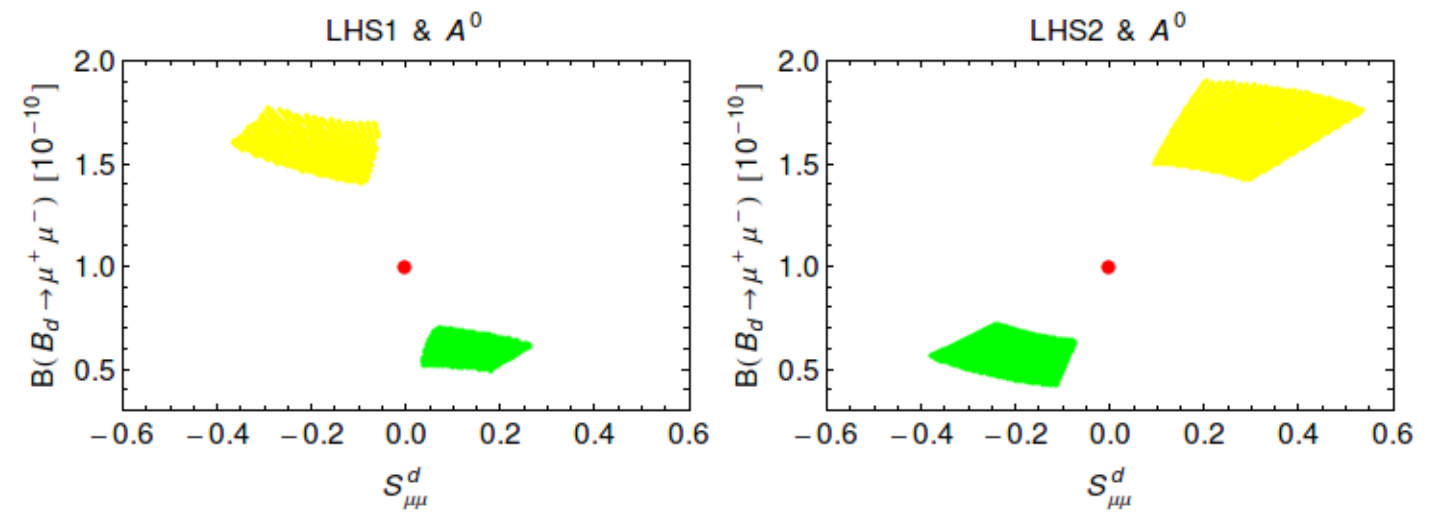

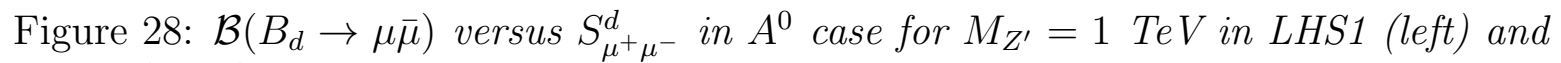
LHS2 (right) for the green and yellow oases as discussed in the text. Red point: SM central value.
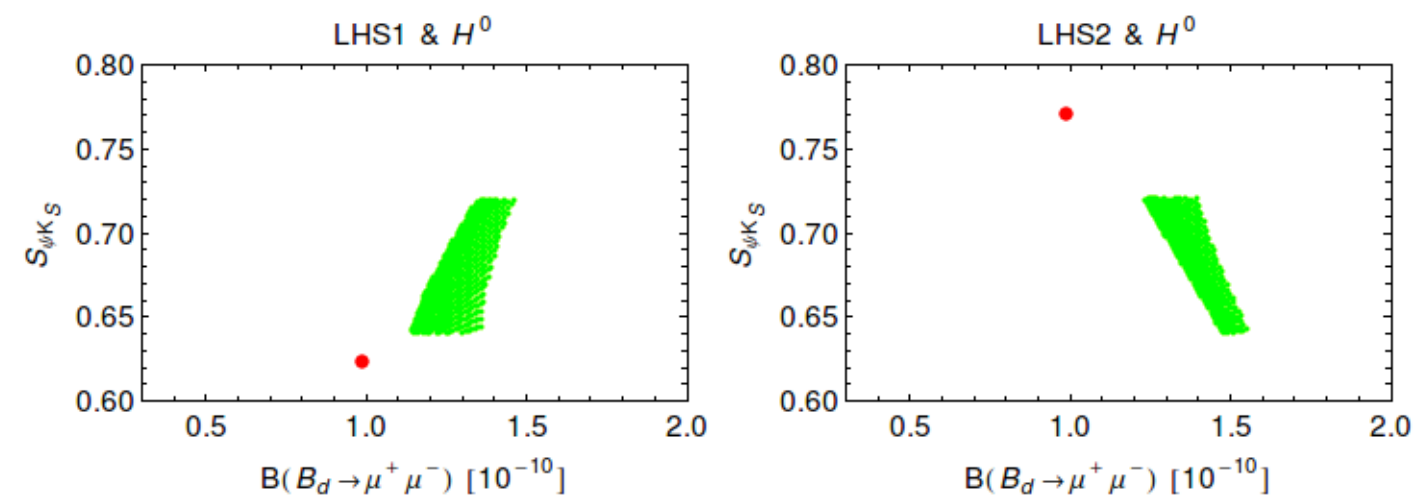

Figure 29: $S_{\psi K_{S}}$ versus $\mathcal{B}\left(B_{d} \rightarrow \mu^{+} \mu^{-}\right)$in $H^{0}$ scenario for $M_{H}=1$ TeV in LHS1 (left) and LHS2 (right) in the yellow and green oases that overlap here. Red point: SM central value.

symmetry. The main effect is on $B_{s} \rightarrow \mu^{+} \mu^{-}$and we have shown it in all plots above. Presently most interesting in this context is the correlation between $S_{\psi \phi}$ and $\mathcal{B}\left(B_{s} \rightarrow\right.$ $\mu^{+} \mu^{-}$). We observe that already the sign of $S_{\psi \phi}$ will decide whether LHS1 or LHS2 is favoured. Moreover if $\mathcal{B}\left(B_{s} \rightarrow \mu^{+} \mu^{-}\right)$will turned out to be suppressed relatively to the SM then only one oasis will survive in each scenario. Comparison with future precise value of $\left|V_{u b}\right|$ will confirm or rule out this scenario of NP. These correlations are particular examples of the correlations in $U(2)^{3}$ models pointed out in [39]. What is new here is that in a specific model considered by us the $\left|V_{u b}\right|-S_{\psi \phi}$ correlation has now also implications not only for $\mathcal{B}\left(B_{s} \rightarrow \mu^{+} \mu^{-}\right)$but also for $S_{\mu \mu}^{s}$ as seen in other plots. Analogous comments can be made in the case of $A^{0}$ and $H^{0}$. 

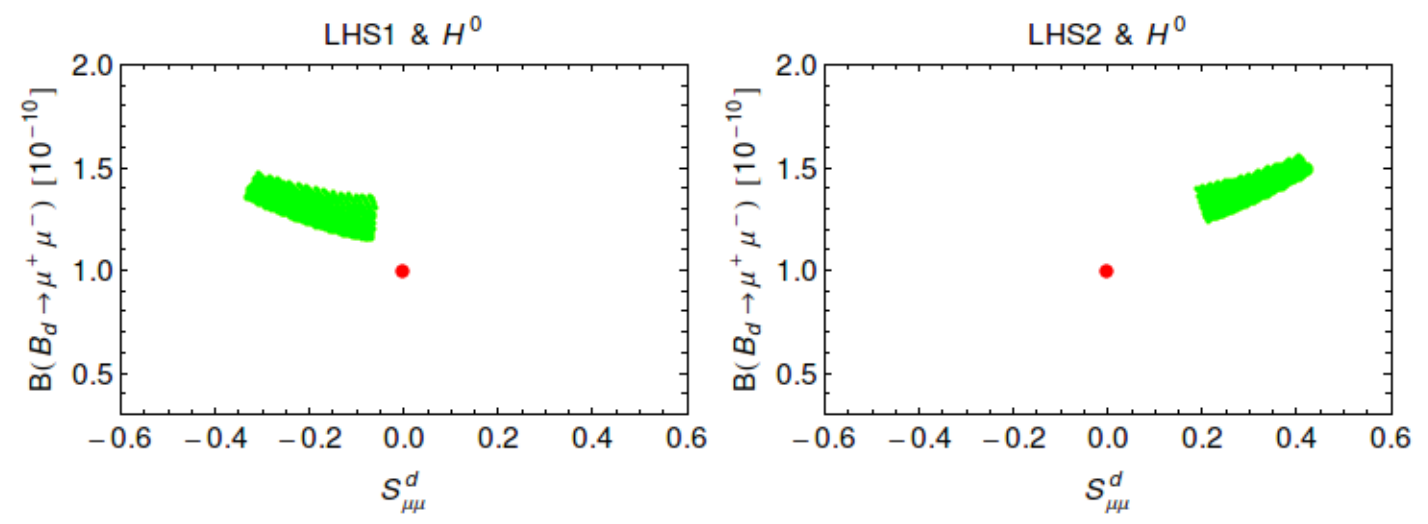

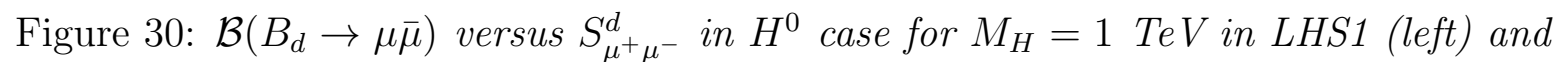
LHS2 (right) for the green and yellow oases (they overlap here) as discussed in the text. Red point: SM central value.

\subsubsection{Dependence of $\Delta F=1$ Transitions on $M_{Z^{\prime}}$}

The nominal value of $M_{Z^{\prime}}$ in the plots presented in this review is $1 \mathrm{TeV}$ except for few cases where higher values are considered. The results for $M_{Z^{\prime}}=3 \mathrm{TeV}$ in 331 models can be found in [41, 47]. Here following [40,47] we would like to summarize how our results for $\Delta F=1$ transitions can be translated into other values of $M_{Z^{\prime}}$ in case higher values would be required by the LHC and other constraints in a given model. In this translation the lepton couplings have to be held fixed.

As presently the constraints on $Z^{\prime}$ models are dominated by $\Delta F=2$ transitions it turns out that for a given allowed size of $\Delta S\left(B_{q}\right)$, NP effects in the one-loop $\Delta F=1$ functions are proportional to $1 / M_{Z^{\prime}}$. That these effects are only suppressed like $1 / M_{Z^{\prime}}$ and not like $1 / M_{Z^{\prime}}^{2}$ is the consequence of the increase with $M_{Z^{\prime}}$ of the allowed values of the couplings $\Delta_{L, R}^{i j}\left(Z^{\prime}\right)$ extracted from $\Delta F=2$ observables. When NP effects are significantly smaller than the SM contribution, only interference between SM and NP contributions matters and consequently this dependence is transfered to the branching ratios. In summary, denoting by $\Delta \mathcal{O}^{\mathrm{NP}}\left(M_{Z^{\prime}}^{(i)}\right) \mathrm{NP}$ contributions to a given $\Delta F=1$ observable in $B_{s}$ and $B_{d}$ decays at two $\left((i=1,2)\right.$ different values $M_{Z^{\prime}}^{(i)}$ we have a scaling law

$$
\Delta \mathcal{O}^{\mathrm{NP}}\left(M_{Z^{\prime}}^{(1)}\right)=\frac{M_{Z^{\prime}}^{(2)}}{M_{Z^{\prime}}^{(1)}} \Delta \mathcal{O}^{\mathrm{NP}}\left(M_{Z^{\prime}}^{(2)}\right) .
$$

This scaling law is valid in most of observables in $B_{s}$ and $B_{d}$ systems as NP effects are bounded to be small. In the rare $K$ decays, like $K^{+} \rightarrow \pi^{+} \nu \bar{\nu}$ and $K_{L} \rightarrow \pi^{0} \nu \bar{\nu}$, where NP contributions for sufficiently low values of $M_{Z^{\prime}}$ could be much larger than the SM contribution, NP modifications of branching ratios will decrease faster than $1 / M_{Z^{\prime}}$ $\left(1 / M_{Z^{\prime}}^{2}\right.$ in the limit of full NP dominance) until NP contributions are sufficiently small 
so that the $1 / M_{Z^{\prime}}$ dependence and (166) is again valid.

Needless to say, when also lepton couplings can be varied in order to compensate for the change of $M_{Z^{\prime}}$, the scaling law could be modified. In this case the correlations between NP corrections to various one-loop functions, derived in [40, 47], are helpful in translating our results into the ones obtained for different $M_{Z^{\prime}}$ and lepton couplings. We refer in particular to [47] where using the data from LEP-II, CMS and ATLAS the bounds on $M_{Z^{\prime}}$ in various 331 models with different lepton couplings have been analyzed.

\subsubsection{Flavour Violating SM $Z$ and SM Higgs Boson}

Let us next look at a possibility that NP will only be detectable through modified $Z$ and Higgs couplings. Beginning with flavour violating $Z$-couplings they can be generated in the presence of other neutral gauge bosons and or new heavy vectorial fermions with $+2 / 3$ and $-1 / 3$ electric charges. RSc is an explicit model of this type 49, 167 (see also [168|). Recently, an extensive analysis of flavour violation in the presence of a vectorial $+2 / 3$ quark has been presented in [169], where references to previous literature can be found.

The formalism developed for $Z^{\prime}$ can be used directly here by setting

$$
M_{Z}=91.2 \mathrm{GeV}, \quad \Delta_{L}^{\nu \bar{\nu}}(Z)=\Delta_{A}^{\mu \bar{\mu}}(Z)=0.372, \quad \Delta_{V}^{\mu \bar{\mu}}(Z)=-0.028
$$

The implications of these changes are as follows:

- The decrease of the neutral gauge boson mass by an order of magnitude relatively to the nominal value $M_{Z^{\prime}}=1 \mathrm{TeV}$ used by us decreases the couplings $\tilde{s}_{i j}$ by the same amount without any impact on the phases $\delta_{i j}$ when the constraints from $\Delta F=2$ processes are imposed.

- As pointed out in [40] once the parameters $\tilde{s}_{i j}$ are constrained through $\Delta F=2$ observables the decrease of neutral gauge boson mass enhances NP effects in rare $K$ and $B$ decays. This follows from the structure of tree-level contributions to FCNC processes and is not generally the case when NP contributions are governed by penguin and box diagrams.

- The latter fact implies that already the present experimental bounds on $\mathcal{B}\left(K^{+} \rightarrow\right.$ $\left.\pi^{+} \nu \bar{\nu}\right)$ and $\mathcal{B}\left(B_{s, d} \rightarrow \mu^{+} \mu^{-}\right)$as well as the data on $B \rightarrow X_{s} \ell^{+} \ell^{-}, B \rightarrow K^{*} \ell^{+} \ell^{-}$ and $B \rightarrow K \ell^{+} \ell^{-}$decays become more powerful than the $\Delta F=2$ transitions in constraining flavour violating couplings of $Z$ so that effects in $\Delta F=2$ processes cannot be as large as in $Z^{\prime}$ case.

The patterns of flavour violation through $Z$ in $B_{s}, B_{d}$ and $K$ are strikingly different from each other: 
- In the $B_{s}$ system when the constraints from $\Delta M_{s}$ and $S_{\psi \phi}$ are imposed $\mathcal{B}\left(B_{s} \rightarrow\right.$ $\mu^{+} \mu^{-}$) is always larger than its SM value and mostly above the data except in LRS case where NP contributions vanish. Further constraints follow from $b \rightarrow s \ell^{+} \ell^{-}$transitions so that one has to conclude that it is very difficult to suppress $\Delta M_{s}$ sufficiently in LHS, LRS and RHS scenarios without violating the constraints from $b \rightarrow s \mu^{+} \mu^{-}$transitions. Thus we expect $\mathcal{B}\left(B_{s} \rightarrow \mu^{+} \mu^{-}\right)$to be enhanced over the SM value but simultaneously possible tension in $\Delta M_{s}$ cannot be solved if the relevant parameters are like in (36). Future lattice calculations will tell us whether this is indeed a problem. Similar conclusions have been reached in [38, 170]. Yet, as demonstrated recently in [46] by changing the nonperturbative parameters agreement with both data on $\Delta F=2$ observables and $B_{s} \rightarrow \mu^{+} \mu^{-}$can be obtained and we will summarize this analysis below.

- In the $B_{d}$ system all $\Delta F=2$ constraints can be satisfied. We again observe that $\mathcal{B}\left(B_{d} \rightarrow \mu^{+} \mu^{-}\right)$can be enhanced by almost an order magnitude and this begins to be a problem for certain choices of couplings in view of recent $\mathrm{LHCb}$ and CMS data. This is shown in Fig. 31 for the LHS1 and LHS2 scenarios. Evidently NP effects are much larger than in the $Z^{\prime}$ case. We also show the results in ALRS1 and ALRS2 scenarios in which NP effects are smaller than in LHS1 and LHS2 scenarios. With improved upper bound on $\mathcal{B}\left(B_{d} \rightarrow \mu^{+} \mu^{-}\right)$LHS1 and LHS2 scenarios could be put into difficulties, while in ALRS1 and ALRS2 one could easier satisfy this bound. If such a situation really took place and NP effects would be observed in this decay, this would mean that both LH and RH $Z$-couplings in the $B_{d}$ system would be required but with opposite sign.

- As we will see in Step 8, the effects of flavour violating $Z$ couplings in $K^{+} \rightarrow \pi^{+} \nu \bar{\nu}$ and $K_{L} \rightarrow \pi^{0} \nu \bar{\nu}$ can be in principle very large in LHS, RHS and LRS scenarios but they can be bounded by the upper bound on $K_{L} \rightarrow \mu^{+} \mu^{-}$except for the LR scenarios and the case of purely imaginary NP contributions in all these scenarios where this bound is ineffective. We show in Step 8 in Fig. 39 few examples which demonstrate that even with the latter constraint taken into account flavour violating $Z$ can have impact on rare $K$ decays which is significantly larger than in the $Z^{\prime}$ case.

In summary flavour-violating $Z$ couplings in $B_{d} \rightarrow \mu^{+} \mu^{-}$decay, similarly to $Z^{\prime}$ couplings in rare $K$ decays discussed in Step 8, could turn out to be an important portal to short distance scales which cannot be explored by the LHC. For $B_{s} \rightarrow \mu^{+} \mu^{-}$decay this does not seem to be the case any longer.

Concerning the tree-level SM Higgs contributions to FCNCs one finds that once the constraints on flavour-violating couplings from $\Delta F=2$ observables are imposed, the smallness of Higgs couplings to muons precludes any measurable effects in $\mathcal{B}\left(B_{d} \rightarrow \mu^{+} \mu^{-}\right)$ and $\mathcal{B}\left(B_{s} \rightarrow \mu^{+} \mu^{-}\right)$can be only enhanced by at most $8 \%$ [44]. Still the presence of such contributions can remove all possible tensions within the SM in $\Delta F=2$ transitions without being in conflict with constraints from rare decays. 

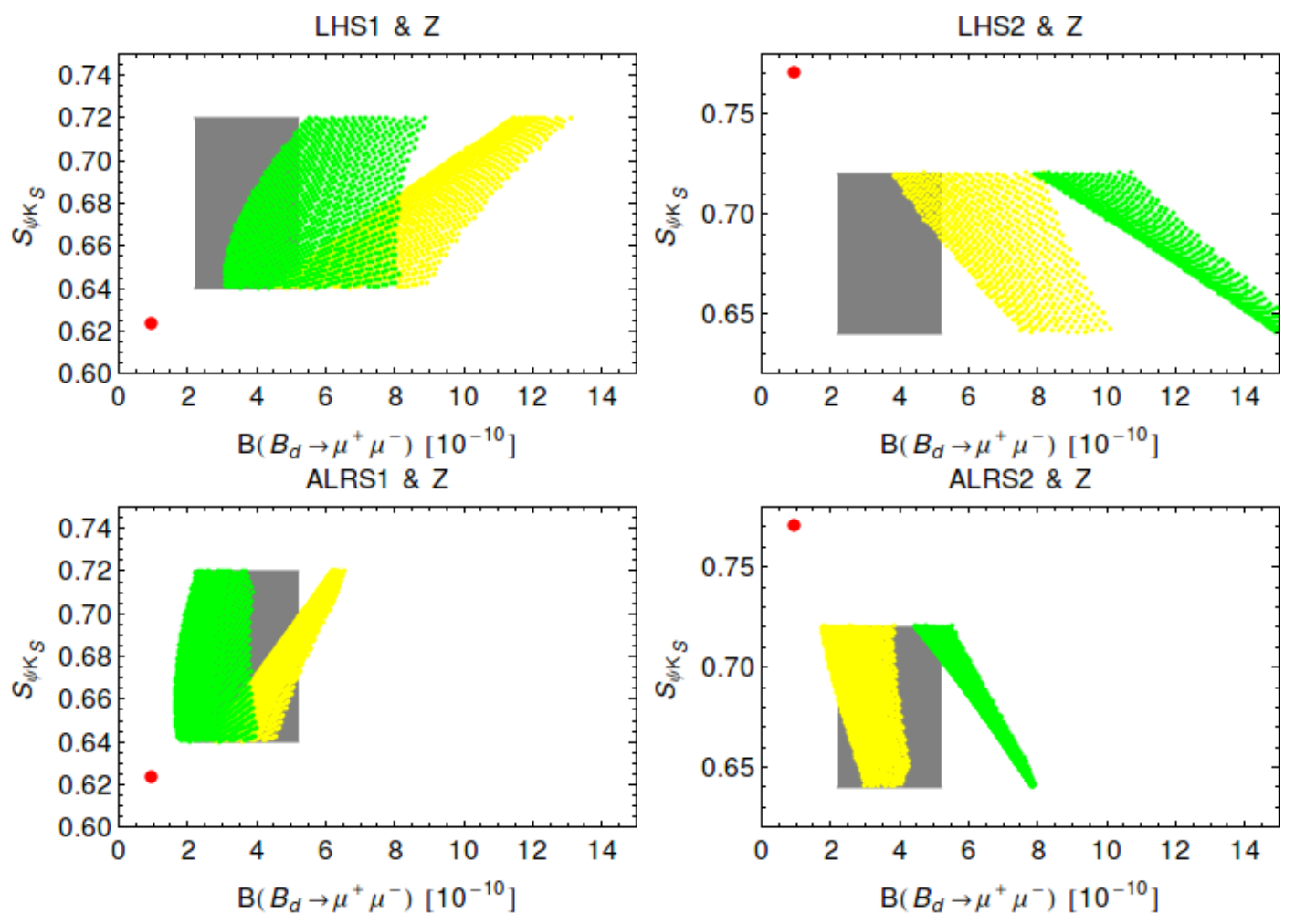

Figure 31: $S_{\psi K_{S}}$ versus $\mathcal{B}\left(B_{d} \rightarrow \mu^{+} \mu^{-}\right)$in LHS1, LHS2 (upper row) and ALRS1, ALRS2 (lower row). $B_{1}$ : yellow, $B_{3}$ : green. Red point: SM central value. Gray region: $1 \sigma$ range of $\mathcal{B}\left(B_{d} \rightarrow \mu^{+} \mu^{-}\right)=\left(3.6_{-1.4}^{+1.6}\right) \cdot 10^{-10}$ and $2 \sigma$ region of $S_{\psi K_{S}} \in[0.639,0.719]$.

Similarly to modifications of $Z$ and SM Higgs couplings, also couplings of $W^{ \pm}$could be modified by NP. There are many papers studying implications of such modifications for FCNC processes. We refer to the recent detailed analysis in [171], where further references can be found. In particular the constraints on the anomalous $t W b$ interactions turn out to be superior to present direct constraints from top decays and production measurements at Tevatron and the LHC.

\subsubsection{Facing the violation of CMFV Relation (14)}

As shown in Fig. 17 the stringent CMFV relation in (14) appears to be violated by the present data. Even if this violation is still not statistically significant in view of very inaccurate data on $B_{d} \rightarrow \mu^{+} \mu^{-}$it is of interest to see whether tree-level exchanges of $Z^{\prime}$ and $Z$ could with a certain choices of quark and lepton couplings reproduce these data while satisfying $\Delta F=2$ constraints and the constraints from $B_{d} \rightarrow K^{*}(K) \mu^{+} \mu^{-}$ considered in Step 7. As in the numerical analysis presented sofar NP in $\Delta F=2$ processes was governed by $(36)$ and consequently $C_{B_{s}} \approx C_{B_{d}} \approx 0.93$, it is also interesting 

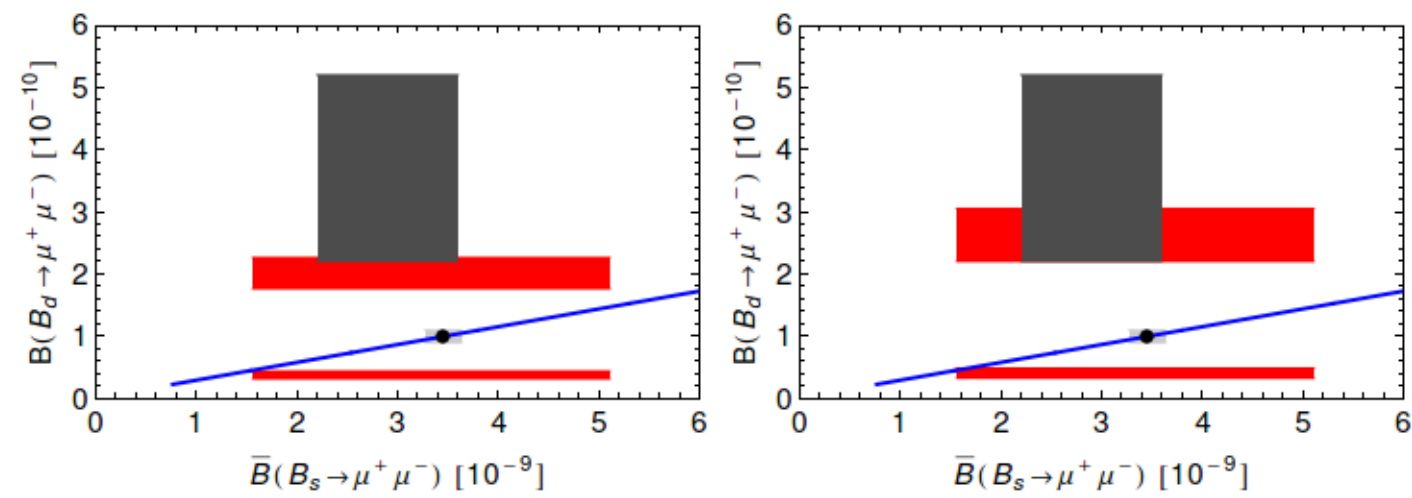

Figure 32: $\quad \mathcal{B}\left(B_{d} \rightarrow \mu^{+} \mu^{-}\right)$versus $\overline{\mathcal{B}}\left(B_{s} \rightarrow \mu^{+} \mu^{-}\right)$in the $Z^{\prime}$ scenario for $\left|V_{u b}\right|=$ 0.0034 (left) and $\left|V_{u b}\right|=0.0040$ (right) and $C_{B_{d}}=1.04 \pm 0.01, C_{B_{s}}=1.00 \pm 0.01$, $\bar{\Delta}_{A}^{\mu \bar{\mu}}=1 \mathrm{TeV}^{-1}, 0.639 \leq S_{\psi K_{s}} \leq 0.719$ and $-0.15 \leq S_{\psi \phi} \leq 0.15$. SM is represented by the light gray area with black dot and the CMFV prediction by the blue line. Dark gray region: Combined exp $1 \sigma$ range $\overline{\mathcal{B}}\left(B_{s} \rightarrow \mu^{+} \mu^{-}\right)=(2.9 \pm 0.7) \cdot 10^{-9}$ and $\mathcal{B}\left(B_{d} \rightarrow \mu^{+} \mu^{-}\right)=\left(3.6_{-1.4}^{+1.6}\right) \cdot 10^{-10}$.

to see what happens when these values are modified.

Such an analysis has been recently performed in [46] concentrating on the LHS scenario, which as discussed in Step 7 gives a plausible explanation of the $B_{d} \rightarrow K^{*}(K) \mu^{+} \mu^{-}$ data. Its outcome can be briefly summarized as follows:

- The LHS scenario for $Z^{\prime}$ or $Z$ FCNC couplings provides a simple model that allows for the violation of the CMFV relation between the branching ratios for $B_{d, s} \rightarrow \mu^{+} \mu^{-}$and $\Delta M_{s, d}$. The plots in Figs. 32 and 33 for $Z^{\prime}$ and $Z$, respectively, illustrate this.

- However, to achieve this in the case of $Z^{\prime}$ the experimental value of $\Delta M_{s}$ must be very close to its $\mathrm{SM}$ value $\left(C_{B_{s}}=1.00 \pm 0.01\right)$ and $\Delta M_{d}$ is favoured to be a bit larger than $\left(\Delta M_{d}\right)_{\mathrm{SM}}\left(C_{B_{d}}=1.04 \pm 0.01\right) . S_{\psi \phi}$ can still deviate significantly from its SM value.

- In the case of $Z$, both $\Delta M_{s}$ and $S_{\psi \phi}$ must be rather close to their SM values while $\Delta M_{d}$ is favoured to be smaller than $\left(\Delta M_{d}\right)_{\mathrm{SM}}\left(C_{B_{d}}=0.96 \pm 0.01\right)$.

In [46] details on the dependence of the correlation between branching ratios for $B_{s, d} \rightarrow$ $\mu^{+} \mu^{-}$and the CP-asymmetries $S_{\psi \phi}$ and $S_{\psi K_{S}}$ on the values of $C_{B_{s}}$ and $C_{B_{d}}$ can be found. Also the anatomy of the plots in Figs. 32 and 33 is presented there. With the improved data and increased lattice calculations such plots will be more informative than presently. 

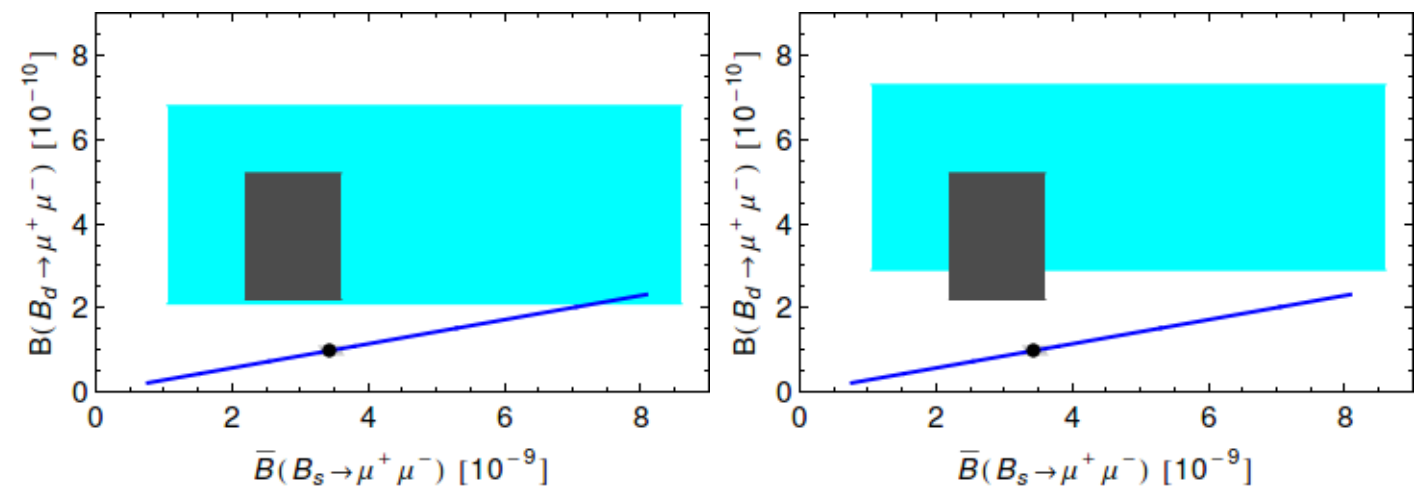

Figure 33: $\mathcal{B}\left(B_{d} \rightarrow \mu^{+} \mu^{-}\right)$versus $\overline{\mathcal{B}}\left(B_{s} \rightarrow \mu^{+} \mu^{-}\right)$in the $Z$-scenario for $\left|V_{u b}\right|=0.0034$ (left) and $\left|V_{u b}\right|=0.0040$ (right) and $C_{B_{d}}=0.96 \pm 0.01, C_{B_{s}}=1.00 \pm 0.01,0.639 \leq$ $S_{\psi K_{s}} \leq 0.719$ and $-0.15 \leq S_{\psi \phi} \leq 0.15$. SM is represented by the light gray area with black dot. Dark gray region: Combined exp $1 \sigma$ range $\overline{\mathcal{B}}\left(B_{s} \rightarrow \mu^{+} \mu^{-}\right)=(2.9 \pm 0.7) \cdot 10^{-9}$ and $\mathcal{B}\left(B_{d} \rightarrow \mu^{+} \mu^{-}\right)=\left(3.6_{-1.4}^{+1.6}\right) \cdot 10^{-10}$.

\subsubsection{2 $\mathcal{B}\left(B_{s} \rightarrow \mu^{+} \mu^{-}\right)$as an Electroweak Precision Test}

Our review deals dominantly with flavour violation. Yet, in particular NP models relations between flavour violating and flavour conserving couplings exist so that additional correlations between flavour violating and flavour conserving processes are present. Such correlations can involve on one hand left-handed $Z b \bar{b}$ and $Z b \bar{s}$ couplings and on the other hand corresponding right-handed couplings. In particular it is known that the measured right-handed $Z b \bar{b}$ coupling disagrees with its SM value by $3 \sigma$. The physics responsible for this anomaly can in some NP models through correlations also have an impact on FCNC processes.

Such a correlation has been pointed out first in [172], and analyzed in detail in the context of MFV in 173 . At that time the information on $Z \rightarrow b \bar{b}$ couplings was by far superior to the one from $B_{s} \rightarrow \mu^{+} \mu^{-}$so that the bounds on possible deviations of $Z \rightarrow b \bar{b}$ from their SM values implied interesting bounds on FCNC processes, including $B_{s} \rightarrow$ $\mu^{+} \mu^{-}$. As pointed out recently in $[142$ the situation is now reversed and the present data on $\mathcal{B}\left(B_{s} \rightarrow \mu^{+} \mu^{-}\right)$set already the dominant constraints on possible modified flavour diagonal $Z$-boson couplings. In the case of MFV models, where significant NP effects are expected only in LH $Z$ - couplings, the present bound derived in 142 from $\mathcal{B}\left(B_{s} \rightarrow \mu^{+} \mu^{-}\right)$is not much stronger than the one derived from $Z \rightarrow b \bar{b}$. On the other hand in generic models with partial compositeness $\mathcal{B}\left(B_{s} \rightarrow \mu^{+} \mu^{-}\right)$sets already now constraint on the $\mathrm{RH} Z b \bar{b}$ coupling that is significantly more stringent than obtained from $Z \rightarrow b \bar{b}$. As a result, in this class of models the present anomaly in $\mathrm{RH} Z b \bar{b}$ coupling cannot be explained. Needless to say, such constraints on diagonal $Z b \bar{b}$ coupling will become even more powerful when the measurement of $\mathcal{B}\left(B_{s} \rightarrow \mu^{+} \mu^{-}\right)$improves so that this decay will offer electroweak precision tests. 


\subsubsection{3 $\quad B_{s, d} \rightarrow \tau^{+} \tau^{-}$}

The leptonic decays $B_{s, d}^{0} \rightarrow \tau^{+} \tau^{-}$could one day play a significant role in the tests of NP models. In particular interesting information on the interactions of new particles with the third generation of quarks and leptons could be obtained in this manner. In the SM the branching ratios in question are enhances by roughly two orders of magnitude over the corresponding decays to the muon pair:

$$
\frac{\mathcal{B}\left(B_{q} \rightarrow \tau^{+} \tau^{-}\right)}{\mathcal{B}\left(B_{q} \rightarrow \mu^{+} \mu^{-}\right)}=\sqrt{1-\frac{4 m_{\tau}^{2}}{m_{B_{q}}^{2}}} \frac{m_{\tau}^{2}}{m_{\mu}^{2}} \approx 210 .
$$

Tree-level exchange of a neutral SM Higgs with quark flavour violating couplings could become important and the same applies to tree-level heavy scalar and pseudoscalar exchanges. There are presently no experimental limits on these decays, however the interplay with $\Gamma_{12}^{s}$, and the latest measurements of $\Gamma_{d} / \Gamma_{s}$ by LHCb would imply the upper bound for branching ratio for $B_{s}^{0} \rightarrow \tau^{+} \tau^{-}$of $3 \%$ at $90 \%$ C.L. [174, 175]. Due to significant experimental challenges to observe these decays at the $\mathrm{LHCb}$ it is then unlikely that we will benefit from them in this decade and we will not discuss them further.

\subsection{Step 5: $B^{+} \rightarrow \tau^{+} \nu_{\tau}$}

\subsubsection{Preliminaries}

We now look at the tree-level decay $B^{+} \rightarrow \tau^{+} \nu$, which was the subject of great interest in the previous dacade as the data from BaBar [176] and Belle [177] implied a world average in the ballpark of $\mathcal{B}\left(B^{+} \rightarrow \tau^{+} \nu_{\tau}\right)_{\exp }=(1.73 \pm 0.35) \times 10^{-4}$, roughly by a factor of 2 higher than the SM value. Meanwhile, the situation changed considerably due to 2012 data from Belle [178] so that the present world average that combines BaBar and Belle data reads $[112$

$$
\mathcal{B}\left(B^{+} \rightarrow \tau^{+} \nu_{\tau}\right)_{\exp }=(1.14 \pm 0.22) 10^{-4},
$$

which is fully consistent with the values quoted in Table 4 with some preference for the inclusive values of $\left|V_{u b}\right|$. Yet, the rather large experimental error and parametric uncertainties in the SM prediction still allow in principle for sizable NP contributions.

In this context one should recall that one of our working assumptions was the absence of significant NP contributions to decays governed by tree-diagrams. Yet the decay in question could be one of the exceptions as it is governed by the smallest element of the CKM matrix $\left|V_{u b}\right|$ and its branching ratio is rather small for a tree-level decay. We will therefore briefly discuss it in the simplest extensions of the SM.

The motivation for this study is the sensitivity of this decay to new heavy charged gauge bosons and scalars that we did not encounter in the previous steps, where neutral gauge bosons and neutral scalars and pseudoscalars dominated the scene. 


\subsubsection{Standard Model Results}

In the SM $B^{+} \rightarrow \tau^{+} \nu_{\tau}$ is mediated by the $W^{ \pm}$exchange with the resulting branching ratio given by

$\mathcal{B}\left(B^{+} \rightarrow \tau^{+} \nu_{\tau}\right)_{\mathrm{SM}}=\frac{G_{F}^{2} m_{B^{+}} m_{\tau}^{2}}{8 \pi}\left(1-\frac{m_{\tau}^{2}}{m_{B^{+}}^{2}}\right)^{2} F_{B^{+}}^{2}\left|V_{u b}\right|^{2} \tau_{B^{+}}=6.05\left|V_{u b}\right|^{2}\left(\frac{F_{B^{+}}}{185 \mathrm{MeV}}\right)^{2}$.

Evidently this result is subject to significant parametric uncertainties induced in (170) by $F_{B^{+}}$and $\left|V_{u b}\right|$. However, recently the error on $F_{B^{+}}$from lattice QCD decreased significantly so that the dominant uncertainty comes from $\left|V_{u b}\right|$. Indeed, as seen in Table 4, for fixed remaining input parameters, varying $\left|V_{u b}\right|$ in the range shown in this table modifies the branching ratio by roughly a factor of two.

In the literature in order to find the SM prediction for this branching ratio one eliminates these uncertainties by using $\Delta M_{d}, \Delta M_{d} / \Delta M_{s}$ and $S_{\psi K_{S}}$ [35, 179] and taking experimental values for these three quantities. To this end $F_{B^{+}}=F_{B_{d}}$ is assumed in agreement with lattice values. This strategy has a weak point as the experimental values of $\Delta M_{d, s}$ used in this strategy may not be the ones corresponding to the true value of the SM. However, proceeding in this manner one finds [35]

$$
\mathcal{B}\left(B^{+} \rightarrow \tau^{+} \nu\right)_{\mathrm{SM}}=(0.80 \pm 0.12) \times 10^{-4},
$$

with a similar result obtained by the UTfit collaboration [179]. As seen in Table 4 this result corresponds to $\left|V_{u b}\right|$ in the ballpark of $3.6 \times 10^{-3}$ and is fully consistent with the data in 169).

Unfortunately, the full clarification of a possible presence of NP in this decay will have to wait for the data from SuperKEKB. In the meantime hopefully the error on $F_{B}^{+}$from lattice QCD will be further reduced and theoretical advances in the determination of $\left|V_{u b}\right|$ from tree level decays will be made allowing us to make a precise prediction for this decay without using the experimental value for $\Delta M_{d}$.

It should be emphasized that for low value of $\left|V_{u b}\right|$ the increase of $F_{B^{+}}$, while enhancing the branching ratio in question, would also enhance $\Delta M_{d}$ which in view of our discussion in Step 3 is not favoured by the data. On the other hand the increase of $\left|V_{u b}\right|$ while enhancing $\mathcal{B}\left(B^{+} \rightarrow \tau^{+} \nu\right)_{\text {SM }}$ would also enhance $S_{\psi K_{S}}$ shifting it away from the data. This discussion shows clearly that before all these parameters will be known significantly more precisely than it is the case now, it will be difficult to use this decay for the identification of NP. In fact the decays $B_{s, d} \rightarrow \mu^{+} \mu^{-}$are presently in a much better shape than $B^{+} \rightarrow \tau^{+} \nu$ as they are governed by $\left|V_{t s}\right|$, which is presently much better known than $\left|V_{u b}\right|$.

In view of this uncertain situation our look at the simplest models providing new contributions to this decay will be rather brief. 


\subsubsection{CMFV}

To our knowledge $B^{+} \rightarrow \tau^{+} \nu_{\tau}$ decay has never been considered in CMFV. Here we would like to point out that in this class of models the branching ratio for this decay is enhanced (suppressed) for the same (opposite) sign of the lepton coupling of the new charged gauge boson relative to the SM one. Indeed, the only possibility to modify the $\mathrm{SM}$ result up to loop corrections in CMFV is through a tree-level exchange of a new charged gauge boson, whose flavour interactions with quarks are governed by the CKM matrix. In particular the operator structure is the same.

Denoting this gauge boson by $W^{\prime}$ and the corresponding gauge coupling by $\tilde{g}_{2}$ one has

$$
\frac{\mathcal{B}\left(B^{+} \rightarrow \tau^{+} \nu\right)}{\mathcal{B}\left(B^{+} \rightarrow \tau^{+} \nu\right)^{\mathrm{SM}}}=\left(1+r \frac{\tilde{g}_{2}^{2}}{g_{2}^{2}} \frac{M_{W}^{2}}{M_{W^{\prime}}^{2}}\right)^{2}
$$

where we introduced a factor $r$ allowing a modification in the lepton couplings relatively to the SM ones, in particular of its sign. Which sign is favoured will be known once the data and SM prediction improve.

If $W^{\prime}$ with these properties is absent, the branching ratio in this framework is not modified with respect to the SM up to loop corrections that could involve new particles but are expected to be small. A $H^{ \pm}$exchange generates new operators and is outside this framework. The same comment applies to gauge bosons with right-handed couplings that we will discuss below.

\subsection{4 $2 \mathrm{HDM}_{\overline{\mathrm{MFV}}}$}

Interestingly, when the experimental branching ratio was significantly above its SM value, the tension between theory and experiment in the case of $\mathcal{B}\left(B^{+} \rightarrow \tau^{+} \nu\right)$ increased in the presence of a tree level $H^{ \pm}$exchange. Indeed such a contribution interferes destructively with the $W^{ \pm}$contribution if there are no new sources of CP-violation. This effect has been calculated long time ago by Hou [180] and in modern times calculated first by Akeroyd and Recksiegel [181], and later by Isidori and Paradisi [182] in the context of the MSSM. The same expression is valid in $2 \mathrm{HDM}_{\overline{\mathrm{MFV}}}$ framework and is given as follows 183

$$
\mathcal{B}\left(B^{+} \rightarrow \tau^{+} \nu\right)_{2 \mathrm{HDM}_{\overline{\mathrm{MFV}}}}=\mathcal{B}\left(B^{+} \rightarrow \tau^{+} \nu\right)_{\mathrm{SM}}\left[1-\frac{m_{B}^{2}}{m_{H^{ \pm}}^{2}} \frac{\tan ^{2} \beta}{1+\left(\epsilon_{0}+\epsilon_{1}\right) \tan \beta}\right]^{2} .
$$

In the MSSM $\epsilon_{i}$ are calculable in terms of supersymmetric parameters. In $2 \mathrm{HDM}_{\overline{\mathrm{MFV}}}$ they are just universal parameters that can enter other formulae implying correlations between various observables. If $\epsilon_{i}$ are real, positive definite numbers, similarly to MSSM, also in this model this branching ratio can be strongly suppressed unless the choice of model parameters is such that the second term in the parenthesis is larger than 2. Such a possibility that would necessarily imply a light charged Higgs and large $\tan \beta$ values 
seems to be very unlikely in view of the constraints from other observables as stressed in the past in the context of MSSM in [184] and more recently in the context of the $2 \mathrm{HDM}_{\overline{\mathrm{MFV}}}$ in [183].

However, Isidori and Blankenburg point out that in $2 \mathrm{HDM}_{\overline{\mathrm{MFV}}}$, where $\epsilon_{0}$ and $\epsilon_{1}$ are complex numbers

$$
1+\left(\epsilon_{0}+\epsilon_{1}\right) \tan \beta \leq 0
$$

is possible provided $\tan \beta$ is large. But then these authors find $\mathcal{B}\left(B \rightarrow X_{s} \gamma\right)$ to be suppressed relative to the SM which is not favoured by the data. We will discuss this issue in the next step.

Let us stress in this context that the subscript "SM" in (173) could be misleading as what is really meant there, is the formula for this decay in the SM. While the SM selects the low (exclusive) value for $\left|V_{u b}\right|$ in order to be in agreement with the experimental value of $S_{\psi K_{S}}$, the $2 \mathrm{HDM}_{\overline{\mathrm{MFV}}}$ chooses the large (inclusive) value of $\left|V_{u b}\right|$ in order to be consistent with experimental value of $\varepsilon_{K}$. The resulting problem with $S_{\psi K_{S}}$ is then solved as discussed in Step 3 by new phases in $B_{d}^{0}-\bar{B}_{d}^{0}$ mixing. But with the inclusive value of $\left|V_{u b}\right|, \mathcal{B}\left(B^{+} \rightarrow \tau^{+} \nu\right)$ is enhanced and as seen in Table 4 agreement with the data can be obtained.

It appears then that the simplest solution to the possible problem with $\mathcal{B}\left(B^{+} \rightarrow \tau^{+} \nu\right)$ in this model is the absence of relevant charged Higgs contributions to this decay and sufficiently large value of $\left|V_{u b}\right|$.

\subsubsection{Tree-Level Charged Gauge Boson Exchange}

Let us write the effective Hamiltonian for the exchange of a charged gauge bosons $W^{\prime+}$ contributing to $B^{+} \rightarrow \tau^{+} \nu_{\tau}$ as follows

$$
\mathcal{H}_{\text {eff }}=C_{L} O_{L}+C_{R} O_{R},
$$

where

$$
O_{L}=\left(\bar{b} \gamma_{\mu} P_{L} u\right)\left(\bar{\nu}_{\tau} \gamma^{\mu} P_{L} \tau^{-}\right), \quad O_{R}=\left(\bar{b} \gamma_{\mu} P_{R} u\right)\left(\bar{\nu}_{\tau} \gamma^{\mu} P_{L} \tau^{-}\right)
$$

and

$$
C_{L}=C_{L}^{\mathrm{SM}}+\frac{\Delta_{L}^{u b *}\left(W^{\prime+}\right) \Delta_{L}^{\tau \nu}\left(W^{\prime+}\right)}{M_{W^{\prime+}}^{2}}, \quad C_{R}=\frac{\Delta_{R}^{u b *}\left(W^{\prime+}\right) \Delta_{L}^{\tau \nu}\left(W^{\prime+}\right)}{M_{W^{\prime+}}^{2}}
$$

with $C_{L}^{\mathrm{SM}}$ having the same structure as the correction from $W^{\prime+}$ with

$$
\Delta_{L}^{u b}=\frac{g}{\sqrt{2}} V_{u b}, \quad \Delta_{L}^{\nu \tau}=\frac{g}{\sqrt{2}}, \quad \Delta_{R}^{u b}=0 .
$$

The couplings $\Delta_{L, R}^{u b *}\left(W^{\prime+}\right)$ could be complex numbers and contain new sources of flavour violation. 
Then

$$
\mathcal{B}\left(B^{+} \rightarrow \tau^{+} \nu_{\tau}\right)_{\mathrm{W}^{\prime+}}=\frac{1}{64 \pi} m_{B^{+}} m_{\tau}^{2}\left(1-\frac{m_{\tau}^{2}}{m_{B^{+}}^{2}}\right)^{2} F_{B^{+}}^{2} \tau_{B^{+}}\left|C_{R}-C_{L}\right|^{2} .
$$

Evidently in a model like this it is possible to improve the agreement with the data by choosing appropriately the couplings of $W^{\prime+}$.

\subsubsection{Tree-Level Scalar Exchanges}

We have already discussed such exchanges in the context of $2 \mathrm{HDM}_{\overline{\mathrm{MFV}}}$. Here we want to mention for completeness that the decay $B^{+} \rightarrow D^{0} \tau^{+} \nu$ being sensitive to different couplings of $H^{ \pm}$can contribute significantly to this discussion but form factor uncertainties make this decay less theoretically clean. A thorough analysis of this decay is presented in [185] where further references to older papers can be found.

Recently the BABAR collaboration [186] presented improved analyses for the ratios

$$
\mathcal{R}\left(D^{(*)}\right)=\frac{\mathcal{B}\left(B_{d} \rightarrow D^{(*)} \tau \nu\right)}{\mathcal{B}\left(B_{d} \rightarrow D^{(*)} \ell \nu\right)}
$$

finding

$$
\mathcal{R}(D)=0.440 \pm 0.058 \pm 0.042, \quad \mathcal{R}\left(D^{*}\right)=0.332 \pm 0.024 \pm 0.018
$$

where the first error is statistical and the second one systematic. These results disagree by $2.2 \sigma$ and $2.7 \sigma$ with the SM, respectively [187]

$$
\mathcal{R}_{\mathrm{SM}}(D)=0.297 \pm 0.017, \quad \mathcal{R}_{\mathrm{SM}}\left(D^{*}\right)=0.252 \pm 0.003 .
$$

These values update the ones presented first in [188].

This motivated several theoretical analyses of which we just quote four. First the study of these decays in 2HDM of type III [189, 190] and in NP models with general flavour structure [191]. Moreover in 192 2HDM and 3HDM models with the nonminimal flavor violations originating from flavour-dependent gauge interactions have been analyzed. It is to be seen whether this anomaly remains when the data improve. A recent summary of the situation can be found in [193]. In particular 2HDM of type II cannot simultaneously describe the data on $\mathcal{R}(D)$ and $\mathcal{R}\left(D^{*}\right)$ but this is possible in $2 \mathrm{HDM}$ of type III.

In summary it is evident from this discussion that $B^{+} \rightarrow \tau^{+} \nu_{\tau}, B \rightarrow D \tau \nu$ and $B \rightarrow$ $D^{*} \tau \nu$ can play a potential role in constraining NP models. Yet, due to the fact that the data in the case of $B^{+} \rightarrow \tau^{+} \nu_{\tau}$ moved significantly towards the SM and because of large uncertainty in $\left|V_{u b}\right|$, the identification of a concrete NP at work in this decay appears to us presently as a big challenge. The decays $B \rightarrow D \tau \nu$ and $B \rightarrow D^{*} \tau \nu$ seem to be more promising but we have to wait for improved data as well. It looks like in the SuperKEKB era these three decays taken together will be among the stars of flavour physics. 


\subsection{Step 6: $B \rightarrow X_{s} \gamma$ and $B \rightarrow K^{*} \gamma$}

\subsubsection{Standard Model Results}

The radiative decays in question, in particular $B \rightarrow X_{s} \gamma$, played an important role in constraining NP in the last two decades because both the experimental data and also the theory have been already in a good shape for some time.

The Hamiltonian in the SM is given as follows

$$
\mathcal{H}_{\mathrm{eff}}(b \rightarrow s \gamma)=-\frac{4 G_{\mathrm{F}}}{\sqrt{2}} V_{t s}^{*} V_{t b}\left[C_{7 \gamma}\left(\mu_{b}\right) Q_{7 \gamma}+C_{8 G}\left(\mu_{b}\right) Q_{8 G}\right],
$$

where $\mu_{b}=\mathcal{O}\left(m_{b}\right)$. The dipole operators are defined as

$$
Q_{7 \gamma}=\frac{e}{16 \pi^{2}} m_{b} \bar{s}_{\alpha} \sigma^{\mu \nu} P_{R} b_{\alpha} F_{\mu \nu}, \quad Q_{8 G}=\frac{g_{s}}{16 \pi^{2}} m_{b} \bar{s}_{\alpha} \sigma^{\mu \nu} P_{R} T_{\alpha \beta}^{a} b_{\beta} G_{\mu \nu}^{a} .
$$

While we do not show explicitly the four-quark operators in (183) they are very important for decays considered in this step, in particular as far as QCD and electroweak corrections are concerned.

The special role of these decays is that quite generally they are loop generated processes. As such there are sensitive to NP contributions and in contrast to tree-level FCNCs mediated by neutral gauge bosons and scalars depend often on the masses and couplings of new heavy fermions. But of course new heavy gauge bosons and scalars contribute to these decays in many models as well. At the CKM-suppressed level, tree-level $b \rightarrow u \bar{u} s \gamma$ transitions can also contribute but they are small for the photon energy cut-off $1.6 \mathrm{GeV}$ usually used [194.

The NNLO QCD calculations of $\mathcal{B}\left(B \rightarrow X_{s} \gamma\right)$, that involve a very important mixing of dipole operators with current-current operators, have been in the last decade at the forefront of perturbative QCD calculations in weak decays. The first outcome of these efforts, which included the dominant NNLO corrections was already a rather precise prediction within the SM $1954^{4}$

$$
\mathcal{B}\left(B \rightarrow X_{s} \gamma\right)_{\mathrm{SM}}=(3.15 \pm 0.23) \times 10^{-4},
$$

for $E_{\gamma} \geq 1.6 \mathrm{GeV}$. Since then, several new perturbative contributions have been evaluated [194, 196 202]. Most recently, the $Q_{1,2}-Q_{7}$ interference was found in the $m_{c}=0$ limit 203]. An updated NNLO prediction should be available soon.

Also experimentalists made an impressive progress in measuring this branching ratio reaching the accuracy of $6.4 \%$ 112

$$
\mathcal{B}\left(B \rightarrow X_{s} \gamma\right)_{\exp }=(3.43 \pm 0.22) \times 10^{-4} .
$$

\footnotetext{
${ }^{4}$ For a historical account of NLO and NNLO corrections to this decay see [52].
} 
One expects that in this decade the SuperKEKB will reach the accuracy of $3 \%$ so that very precise tests of the SM and its extensions will be possible.

Comparing the theory with experiment we observe that the experimental value is a bit higher than the theory although presently the difference amounts to only $1.2 \sigma$. However, if the experimental and theoretical errors decrease down to $3 \%$ without the change in central values we will be definitely talking about an anomaly and models in which this branching ratio will be enhanced over the SM result will be favoured. Yet, such models have to satisfy other constraints as well.

In principle a very sensitive observable to NP CP violating effects is the direct $\mathrm{CP}$ asymmetry in $b \rightarrow s \gamma$, i.e. $A_{\mathrm{CP}}(b \rightarrow s \gamma)$ [204], because the perturbative contributions within the SM amount to only $+0.5 \%$ 205-207]. Unfortunately, the analysis [208] shows that this asymmetry, similar to other direct CP asymmetries, suffers from hadronic uncertainties originating here in the hadronic component of the photon. These uncertainties lower the predictive power of this observable. Consequently we do not consider this asymmetry as a superstar of flavour physics and will not include it in our investigations. Similar comments apply to the $B \rightarrow X_{d} \gamma$ decay although CP averaged branching ratio could still provide useful results. Yet, we will leave this decay from our discussion as well, as the remaining observables considered in our paper are evidently more effective in the search for NP from the present perspective.

Concerning $B \rightarrow V \gamma$ decay we refer first to two fundamental papers that include NLO QCD corrections 209,210]. While the branching ratios can already offer useful information, even more promising is the time-dependent CP asymmetry in $B \rightarrow K^{*} \gamma$ 211213

$$
\frac{\Gamma\left(\bar{B}^{0}(t) \rightarrow \bar{K}^{* 0} \gamma\right)-\Gamma\left(B^{0}(t) \rightarrow K^{* 0} \gamma\right)}{\Gamma\left(\bar{B}^{0}(t) \rightarrow \bar{K}^{* 0} \gamma\right)+\Gamma\left(B^{0}(t) \rightarrow K^{* 0} \gamma\right)}=S_{K^{*} \gamma} \sin \left(\Delta M_{d} t\right)-C_{K^{*} \gamma} \cos \left(\Delta M_{d} t\right)
$$

In particular $S_{K^{*} \gamma}$ offers a very sensitive probe of right-handed currents. It vanishes for $C_{7 \gamma}^{\prime} \rightarrow 0$ and consequently in the SM being suppressed by $m_{s} / m_{b}$ is very small [213]:

$$
S_{K^{*} \gamma}^{\mathrm{SM}}=(-2.3 \pm 1.6) \% \text {. }
$$

A useful and rather accurate expression for $S_{K^{*} \gamma}$ has been provided in 212

$$
S_{K^{*} \gamma} \simeq \frac{2}{\left|C_{7 \gamma}\right|^{2}+\left|C_{7 \gamma}^{\prime}\right|^{2}} \operatorname{Im}\left(e^{-i \phi_{d}} C_{7 \gamma} C_{7 \gamma}^{\prime}\right)
$$

with Wilson coefficients evaluated at $\mu=m_{b}$ and $\sin \left(\phi_{d}\right)=S_{\psi K_{S}}$.

On the experimental side, while the present value of $S_{K^{*} \gamma}$ is rather inaccurate 214216

$$
S_{K^{*} \gamma}^{\exp }=-0.16 \pm 0.22
$$

the prospects for accurate measurements at SuperKEKB are very good [19]. 
Also isospin asymmetries in $B \rightarrow V \gamma$ provide interesting tests of the SM and of NP. A detailed recent analysis with references to earlier papers can be found in [217]. On the experimental side the isospin asymmetry in $B \rightarrow K^{*} \gamma$ agrees with the SM, while a $2 \sigma$ deviation from the SM is found in the case of $B \rightarrow \rho \gamma[112]$.

\subsection{2 $B \rightarrow X_{s} \gamma$ Beyond the Standard Model}

Our discussion of NP contributions to this decay will be very brief. The latest review can be found in [218] and a detailed analysis of the impact of anomalous $W t b$ couplings has been presented in [219], where further references to earlier literature can be found.

As the SM agrees well with the data, NP contributions can be at most in the ballpark of $20 \%$ at the level of the branching ratio and they should rather be positive than negative. Consequently this decay will mainly bound the parameters of a given extension of the SM. Here we only make a few comments.

It is known that $B \rightarrow X_{s} \gamma$ can bound the allowed range of the values of charged Higgs $\left(H^{ \pm}\right)$mass and of $\tan \beta$ both in 2HDM and the MSSM. In 2HDM II the contribution of $H^{ \pm}$enhances the branching ratio and $M_{H^{ \pm}}$must be larger than $300 \mathrm{GeV}$ for any value of $\tan \beta$. In the MSSM this enhancement can be compensated by chargino contributions and the bound is weaker.

As we already stated and discussed in more detail in 218$]$ the fact that the SM prediction is below the data favours presently the models that allow for an enhancement of the branching ratio and disfavours those in which only suppression is possible. Table 1 in 218] is useful in this respect. In particular,

- In 2HDM II, Littlest Higgs model without T-parity (LH) and RS $\mathcal{B}\left(B \rightarrow X_{s} \gamma\right)$ can only be enhanced and in LHT the enhancement is favoured.

- In MFV SUSY GUTs [220] and in models with universal extra dimensions it can only be suppressed. In particular in the latter case lower bound on the compactification scale $1 / R$ of $600 \mathrm{GeV}$ can be derived 221 224] in this manner.

- In more complicated models like MSSM with MFV, general MSSM and left-right models both enhancements and suppressions are possible.

Another important virtue of this decay is its sensitivity to right-handed (RH) currents. In the case of left-handed (LH) currents the chirality flip, necessary for $b \rightarrow s \gamma$ to occur, can only proceed through the mass of the initial or the final quark. Consequently the amplitude is proportional to $m_{b}$ or $m_{s}$. In contrast, when $\mathrm{RH}$ currents are present, the chirality flip can take place on the internal top quark line resulting in an enhancement factor $m_{t} / m_{b}$ of the NP contribution relatively to the SM one at the level of the amplitude. This is the case of left-right symmetric models in which $B \rightarrow X_{s} \gamma$ has been analyzed by many authors in the past [131,225 234]. In models with heavy 
fermions $(F)$, that couple through RH currents to SM quarks, this enhancement, being proportional to $m_{F} / m_{b}$ can be very large [135] and the couplings in question must be strongly suppressed in order to obtain agreement with the data. This is for instance the case of gauge flavour models which we will briefly describe in Section 6. It should be emphasized that the comments on the $m_{t} / m_{b}$ and $m_{F} / m_{b}$ enhancements apply also for charged and neutral gauge bosons as well as for charged and neutral heavy scalars and pseudoscalars.

\subsection{Step 7: $B \rightarrow X_{s} \ell^{+} \ell^{-}$and $B \rightarrow K^{*}(K) \ell^{+} \ell^{-}$}

\subsubsection{Preliminaries}

While the branching ratios for $B \rightarrow X_{s} \ell^{+} \ell^{-}$and $B \rightarrow K^{*} \ell^{+} \ell^{-}$put already significant constraints on NP, the angular observables, CP-conserving ones like the well known forward-backward asymmetry and CP-violating ones will definitely be useful for distinguishing various extensions of the SM when the data improve. During the last three years, a number of detailed analyses of various CP averaged symmetries $\left(S_{i}\right)$ and $\mathrm{CP}$ asymmetries $\left(A_{i}\right)$ provided by the angular distributions in the exclusive decay $B \rightarrow K^{*}(\rightarrow K \pi) \ell^{+} \ell^{-}$have been performed in [37, 38, 147, 170, 235 242]. In particular the zeros of some of these observables can be accurately predicted. Pioneering experimental analyses performed at BaBar, Belle and Tevatron 243 245 provided already interesting results for the best known forward-backward asymmetry. Yet, the recent data from LHCb 246, 247] surpassed the latter ones in precision demonstrating that the SM is consistent with the present data on the forward-backward asymmetry. On the other hand these decays as we will see below bring new challenges as the data on $A_{i}$ and $S_{i}$ improved last year. Yet in order to reach clear cut conclusions further improvement in the data and the reduction of theoretical uncertainties is necessary. Meanwhile, the present data serve already to bound the parameters in several extensions of the SM.

Compared with previous steps, this one is more challenging as far as the transparency is concerned. Indeed the effective Hamiltonian for these decays involves more local operators and corresponding Wilson coefficients that generally are complex quantities. On the other hand the numerous symmetries $S_{i}$ and asymmetries $A_{i}$ when precisely measured will allow one day a detailed insight into the physics behind the values of the Wilson coefficients in question. In this context it is important to select those $S_{i}$ and $A_{i}$ which are particularly useful for the tests of NP and are not subject to large form factor uncertainties. While significant progress in this direction has been already done in the literature, a more transparent picture will surely emerge once the precision on these angular observables will increase with time. The most recent reviews on various optimal strategies for extraction of NP from angular observables can be found in 248, 249]. Details on these strategies can be found in 236 238, 240,241, 250, 254].

While it appears from the present perspective that the observables in $B_{s, d} \rightarrow \mu^{+} \mu^{-}$decays are subject to smaller hadronic uncertainties than observables considered here, the 
strength of $B \rightarrow K^{*} \mu^{+} \mu^{-}$is not only the presence of several symmetries $S_{i}$ and asymmetries $A_{i}$ or other constructions like $A_{T}^{i}, P_{i}, H_{T}^{i}$ and alike. Indeed, also the presence of an additional variable, the invariant mass of the dilepton $\left(q^{2}\right)$, is an important virtue of these decays. Studying different observables in different $q^{2}$ bins can indeed one day, as stressed in particular in 238, 240, 248, 249], not only help to discover NP, but also to identify it. The most recent study [255] of the so-called primary observables $P_{i}$ and $P_{i}^{\prime}$ introduced in [241,248] in the context of the most recent $\mathrm{LHCb}$ data [247,256] illustrates this in explicit terms and we will return to these data and the related analyses 255, 257] below.

The story of departures of LHCb data from the SM in the decays in question is rather involved but interesting. In particular previous indications for a deviation from SM value of the isospin asymmetry in $B \rightarrow K^{*} \mu^{+} \mu^{-}$decay now disappeared [258]. On the other hand the corresponding asymmetry in $B \rightarrow K \mu^{+} \mu^{-}$decay disagrees presently with the SM [258]. A recent very detailed analysis of the isospin asymmetries in these decays can be found in 217.

On the other hand as pointed out in 255,257 and analyzed in detail sizable departures from the SM expectations in some of the observables $P_{i}$ or $S_{i}$ are seen in most recent LHCb data 247, 256.

In order to have a closer look at these issues we need the effective Hamiltonian for these decays. It is given in (106) with the first term given in (183). The stars in these decays are the Wilson coefficients entering this Hamiltonians. The most important are

$$
C_{7 \gamma}, \quad C_{9}, \quad C_{10}, \quad C_{7 \gamma}^{\prime}, \quad C_{9}^{\prime}, \quad C_{10}^{\prime}
$$

where the primed Wilson coefficients correspond to primed operators obtained through the replacement $P_{L} \leftrightarrow P_{R}$. The scalar and pseudoscalar coefficients are more constrained by $B_{s} \rightarrow \mu^{+} \mu^{-}$decay but we will make few comments on them below.

The values of the coefficients in (191) have been calculated in the SM and in its numerous extensions. Moreover, they have been constrained in model independent analyses in which they have been considered as real or complex parameters. To this end the data on $B \rightarrow X_{s} \gamma, B \rightarrow K^{*} \gamma, B \rightarrow X_{s} \ell^{+} \ell^{-}, B \rightarrow K^{*} \ell^{+} \ell^{-}, B \rightarrow K \ell^{+} \ell^{-}$and $B_{s} \rightarrow \mu^{+} \mu^{-}$have been used. The fact that these coefficients enter universally in a number of observables allows to obtain correlations between their values. We just refer to selected papers which we found particularly useful for our studies of NP. These are [37, 38, 239, 257], where model-independent constraints on NP in $b \rightarrow s$ transitions have been updated and generalized. Further references can be found there and in the text above.

It is useful to consider $B \rightarrow X_{s} \ell^{+} \ell^{-}$decay and $B \rightarrow K^{*} \ell^{+} \ell^{-}$in two different regions of the dilepton invariant mass. The low $q^{2}$ region with $1 \mathrm{GeV}^{2}<q^{2}<6 \mathrm{GeV}^{2}$, considered already for a long time and the high $q^{2}$ region with $q^{2}>14.4 \mathrm{GeV}^{2}$ which became very relevant after theoretical progress made in [259]. First, in these regions one is not sensitive to the $\bar{c} c$ resonances. Moreover while the branching ratios in the high $q^{2}$ region are mainly sensitive to NP contributions to the Wilson coefficients $C_{9}^{(\prime)}$ and $C_{10}^{(\prime)}$, 
the branching ratio in the low $q^{2}$ region also depends strongly on $C_{7 \gamma}^{(\prime)}$. Therefore, one expects some correlation between NP contributions at low $q^{2}$ and those in $B \rightarrow X_{s} \gamma$ decay.

In [37,38] the NP scenarios without important contributions from scalar operators have been considered. Various analyses show that once the experimental upper bound on the branching ratio for $B_{s} \rightarrow \mu^{+} \mu^{-}$has been taken into account, the impact of pseudoscalar operators $O_{P}^{(\prime)}$ on $B \rightarrow X_{s} \ell^{+} \ell^{-}$and $B \rightarrow K^{*}(K) \ell^{+} \ell^{-}$is minor. However, as stressed in 237] when lepton mass effects are taken into account there is one observable among the many measured in $B \rightarrow K^{*} \ell^{+} \ell^{-}$that is sensitive to scalar operators $O_{S}^{(\prime)}$. This is interesting as $B_{s, d} \rightarrow \mu^{+} \mu^{-}$decays involve generally both scalar and pseudoscalar operators. In this sense angular distribution in $B \rightarrow K^{*} \ell^{+} \ell^{-}$allows to probe the scalar sector of a theory beyond the SM, in a way that is theoretically clean and complementary to $B_{s} \rightarrow \mu^{+} \mu^{-}$. We refer for more details to [237], in particular to Fig. 5 of that paper. However, the recent very improved result from LHCb and CMS on $B_{s} \rightarrow \mu^{+} \mu^{-}$in 133 imposed on this figure precludes this study from present perspective.

While $B \rightarrow K^{*} \ell^{+} \ell^{-}$is not as theoretically clean as $B_{s} \rightarrow \mu^{+} \mu^{-}$because of the presence of form factors, recent advances in lattice calculations 260] give some hopes for improvements. This is also the case of $B \rightarrow K \ell^{+} \ell^{-}$, where progress in lattice calculations of the relevant form factors has been reported in 261,262 .

As stressed in particular in [239] a simultaneous consideration of $B \rightarrow K \ell^{+} \ell^{-}$together with $B_{s} \rightarrow \mu^{+} \mu^{-}$provides useful tests of extensions of the SM. Indeed, while $B_{s} \rightarrow \mu^{+} \mu^{-}$is sensitive only to the differences $C_{P}-C_{P}^{\prime}$ and $C_{S}-C_{S}^{\prime}$, the decay $B \rightarrow K \ell^{+} \ell^{-}$is sensitive to their sums $C_{P}+C_{P}^{\prime}$ and $C_{S}+C_{S}^{\prime}$. A very extensive model independent analysis of $C_{P}\left(C_{P}^{\prime}\right)$ and $C_{S}\left(C_{S}^{\prime}\right)$ in the context of the data on $B_{s} \rightarrow \mu^{+} \mu^{-}$ and $B \rightarrow K \ell^{+} \ell^{-}$has been performed in [239]. With improved data a new insight on the importance of scalar and pseudoscalar operators will be possible.

As we already stated above the picture resulting from these analyses is very rich and a brief summary of these sometimes numerically challenging analyses is a challenge in itself. In what follows we will limit our discussion to a number of observations referring to the rich literature for details, in particular to $37,38,239,257$, as the spirit of these papers fits well to our strategies.

\subsubsection{Lessons from Recent Analyses}

The studies of these decays in the SM and its extensions have been the subject of numerous analyses almost for the last twenty years [155, 263 268]. The most recent studies can be found in $37,235,238,240,255,257,269,270]$, where references to the older papers can be found. The progress in the recent years is the inclusion in these analyses of the data on angular observables in $B \rightarrow K^{*} \ell^{+} \ell^{-}$. In the simplest case the allowed ranges in the space of the real or imaginary parts of a pair of Wilson coefficients, or in the complex plane of a single Wilson coefficient are shown. As stressed in [37] the 
conclusions drawn from such studies are only valid if the chosen Wilson coefficients are indeed the dominant ones in a given NP scenario. In fact this is approximately the case in a number of models considered in the literature. Few examples are:

- In MFV models with dominance of $Z$ penguins and without new sources of CP violation only the real parts of $C_{7 \gamma}$ and $C_{10}$ are relevant.

- In MSSM with MFV and flavour blind phases [78], in effective SUSY with flavour blind phases 271] and in effective SUSY with a $U(2)^{3}$ symmetry [86, 87], NP effects in $\Delta B=\Delta S=1$ processes are dominated by complex contributions to $C_{7}$ and $C_{8}$.

The analysis of this type in [37] uses the data on $B \rightarrow K^{*} \mu^{+} \mu^{-}$at low and high $q^{2}$, $B \rightarrow X_{s} \ell^{+} \ell^{-}, B \rightarrow X_{s} \gamma$ and $B \rightarrow K^{*} \gamma$. The resulting Fig. 2 in that paper containing twelve plots depicts the allowed ranges for various pairs of real and/or imaginary parts of chosen Wilson coefficients. While very impressive, such plots are rather difficult to digest at first side. Yet the message from this analysis is clear. Already present data can exclude sign-flips of certain coefficients in certain NP scenarios relative to SM values. Such plots will be more informative when the data improve.

As in many NP models several Wilson coefficients could be affected by new contributions, the authors of [37] perform probably for the first time a global fit of all Wilson coefficients. In this context in addition to the general case, they consider specific examples of NP scenarios similar in spirit to the ones introduced in Section 2. These are the cases of real LH currents, complex LH currents and complex RH currents. Again 32 plots resulting from this study shows the complexity of such analyses. With improved data such plots will be useful for obtaining an insight into the physics involved. Even if some time passed since this analysis has been published the following observations from this global analysis remain valid:

- For $C_{7 \gamma}, C_{9}$ and $C_{10}$ there is little room left for constructive interference of real NP contributions with the SM.

- A flipped sign solution with $C_{7 \gamma} \simeq-C_{7 \gamma}^{\mathrm{SM}}, C_{9} \simeq-C_{9}^{\mathrm{SM}}$, and $C_{10} \simeq-C_{10}^{\mathrm{SM}}$ is allowed by the data.

- Sizable imaginary parts for all coefficients are still allowed.

A detailed study of $\mathrm{CP}$ symmetries and $\mathrm{CP}$ asymmetries in concrete BSM scenarios can also be found in 237]. In particular it has been found that these observables could allow us clear distinction of LHT, general MSSM and MSSM with flavour blind phases (FBMSSM) not only from SM predictions but also among these three scenarios.

This picture could be modified by the most recent $\mathrm{LHCb}$ data [247, 256] on angular observables in $B_{d} \rightarrow K^{*} \mu^{+} \mu^{-}$that show significant departures from SM expectations. 
Moreover, new data on the observable $F_{L}$, consistent with LHCb value in [247] have been presented by CMS [272]. These anomalies in $B_{d} \rightarrow K^{*} \mu^{+} \mu^{-}$triggered recently two sophisticated analyses 255,257] with the goal to understand the data and to indicate what type of new physics could be responsible for these departures from the SM. Both analyses point toward NP contributions in the modified coefficients $C_{7 \gamma}$ and $C_{9}$ with the following shifts with respect to their SM values:

$$
C_{7 \gamma}^{\mathrm{NP}}<0, \quad C_{9}^{\mathrm{NP}}<0
$$

Other possibilities, in particular involving right-handed currents $\left(C_{9}^{\prime}>0\right)$, have been discussed in [257]. Subsequently several other analyses of these data have been presented 46, 47, 273 279]. In particular, a recent comprehensive Bayesian analysis of the authors of [170, 253 in 275] finds that although SM works well, if one wants to interpret the data in extensions of the SM then scenarios in which chirality-flipped operators are included work better than the ones without them. In that case they find that the main NP effect is still in $C_{9}$ and in agreement with [257] find that in the $C_{9}-C_{9}^{\prime}$ plane the $\mathrm{SM}$ point is outside the $2 \sigma$ range.

It should be emphasized at this point that these analyses are subject to theoretical uncertainties, which have been discussed at length in [242, 255, 259, 270, 279 281] and it remains to be seen whether the observed anomalies are only result of statistical fluctuations and/or underestimated error uncertainties. This has been in particular emphasized by the authors of [275] who do not think that without significant improvement of the understanding of $1 / m_{b}$ corrections and reduction of the uncertainties in hadronic form factors it will be possible to convincingly demonstrate the presence of NP in the decays in question.

Assuming that NP is really at work here we have investigated in 46 whether treelevel $Z^{\prime}$ and $Z$-exchanges could simultaneously explain the $B_{d} \rightarrow \overline{K^{*}} \mu^{+} \mu^{-}$anomalies and the most recent data on $B_{s, d} \rightarrow \mu^{+} \mu^{-}$. In this context we have investigated the correlation between these decays and $\Delta F=2$ observables. The outcome of this rather extensive analysis for $B_{s, d} \rightarrow \mu^{+} \mu^{-}$has been already summarized at the end of Step 4. In particular the plots in Figs. 32 and 33 demonstrate that LHS scenario for $Z^{\prime}$ or $Z$ FCNC couplings provides a simple model that allows for the violation of the CMFV relation between the branching ratios for $B_{d, s} \rightarrow \mu^{+} \mu^{-}$and $\Delta M_{s, d}$.

As far as the anomalies in $B \rightarrow K^{*} \mu^{+} \mu^{-}$are concerned

- $Z^{\prime}$ with only left-handed couplings is capable of softening the anomalies in the observables $F_{L}$ and $S_{5}$ in a correlated manner as proposed [255, 257]. However, a better description of the present data is obtained by including also right-handed contributions with the RH couplings of approximately the same magnitude but opposite sign. This is our ALRS scenario. We illustrate this in Fig. 34. This is in agreement with the findings in [257]. Several analogous correlations can be found in [46]. We should emphasize that if $Z^{\prime}$ is the only new particle at scales $\mathcal{O}(\mathrm{TeV})$ than $C_{7 \gamma}^{\mathrm{NP}}$ can be neglected implying nice correlations shown in Fig. 34 . 

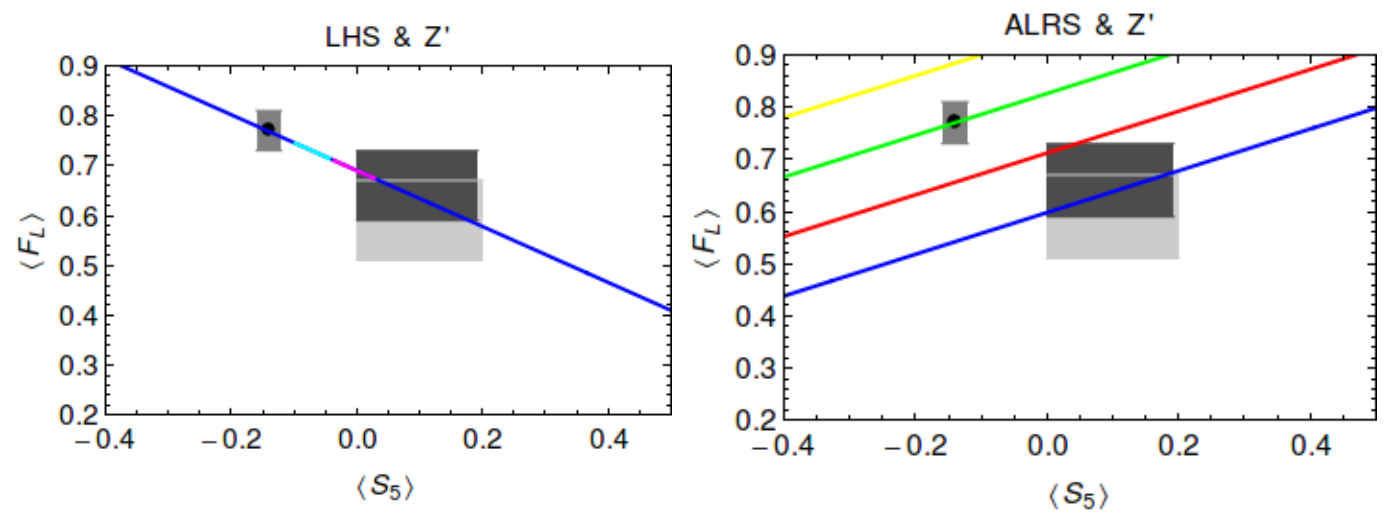

Figure 34: Left: $\left\langle F_{L}\right\rangle$ versus $\left\langle S_{5}\right\rangle$ in LHS where the magenta line corresponds to $C_{9}^{\mathrm{NP}}=$ $-1.6 \pm 0.3$ and the cyan line to $C_{9}^{\mathrm{NP}}=-0.8 \pm 0.3$. Right: The same in ALRS for different values of $C_{9}^{\mathrm{NP}}$ : -2 (blue), -1 (red), 0 (green) and 1 (yellow). The light and dark gray area corresponds to the experimental range for $\left\langle F_{L}\right\rangle$ with all data and only $\mathrm{LHCb}+\mathrm{CMS}$ data, taken into account, respectively. The black point and the gray box correspond to the SM predictions from [257].

- The SM $Z$ boson with FCNC couplings to quarks cannot describe the anomalies in $B \rightarrow K^{*} \mu^{+} \mu^{-}$due to its small vector coupling to muons.

In summary, while the modification of the Wilson coefficient $C_{7 \gamma}$ together with $C_{9}$ could provide the explanation of the data [255, 257], it appears that the most favourite scenario is the one with participation of right-handed currents [46, 257,277]

$$
C_{9}^{\mathrm{NP}}<0, \quad C_{9}^{\prime}>0, \quad C_{9}^{\prime} \approx-C_{9}^{\mathrm{NP}} .
$$

Yet, the case of NP present only in the coefficient $C_{9}$ cannot be presently excluded 46, 47, 255, 273 275. Concerning the dynamics, the favourite physical mechanisms behind these deviations emeraging from these studies is the presence of tree-level $Z^{\prime}$ exchanges. We will summarize the recent results in 331 models [47] in Section 6.5.1.

We are looking forward to improved LHCb data in order to see how the story of NP in $B \rightarrow K^{*}(K) \mu^{+} \mu^{-}$and $B_{s, d} \rightarrow \mu^{+} \mu^{-}$decays evolves with time.

\subsubsection{Explicit Bounds on Wilson Coefficients}

In the present review we have used the results discussed above to constrain the correlations between various observables in models with tree-level neutral gauge boson and neutral scalar and pseudoscalar exchanges. Such constraints can be found in plots presented in Steps 4 and 9. To this end in the case of gauge boson exchanges we use the bounds from Figs. 1 and 2 of [38]. Approximately these bound can be summarized as 
follows:5

$$
\begin{aligned}
& -2 \leq \Re\left(C_{10}^{\prime}\right) \leq 0, \quad-2.5 \leq \Im\left(C_{10}^{\prime}\right) \leq 2.5, \\
& -0.8 \leq \Re\left(C_{10}^{\mathrm{NP}}\right) \leq 1.8, \quad-3 \leq \Im\left(C_{10}\right) \leq 3 .
\end{aligned}
$$

Especially, the LHCb data on $B \rightarrow K^{*} \mu^{+} \mu^{-}$allow only for negative values of the real part of $C_{10}^{\prime}$

$$
\Re\left(C_{10}^{\prime}\right) \leq 0
$$

and this has an impact on our results in RH and LR scenarios presented in Steps 4 and 9. However for the numerical analysis we use the exact bounds that are smaller than these rectangular bounds. For $C_{10}$ - relevant for LHS -the latter allow a much larger region of parameter space whereas for $C_{10}^{\prime}$ - relevant for RHS - the approximation above gives very similar results to the exact bounds in our plots. In Figs. 20, 21, 22, 43 and 44 the green regions in the $Z^{\prime}$ case are compatible with the exact bound from [38]. The black points in RHS show the excluded regions where the bound in 195 is violated which as one can see nearly coincides with the correct bounds (see Figs. 43 and 44 ).

Concerning the bounds on the coefficients of scalar operators we quote here the bounds derived from the analysis in [239]. Adjusting their normalization of Wilson coefficients to ours the final result of this paper reads:

$$
m_{b}\left|C_{S}^{(\prime)}\right| \leq 0.7, \quad m_{b}\left|C_{P}^{(\prime)}\right| \leq 1.0
$$

where the scale in $m_{b}$ should be the high matching scale. As demonstrated in [44] these bounds do not have presently any impact on the values of these coefficients in scenarios with tree-level scalar and pseudoscalar exchanges.

In summary this step will definitely bring new insight into short distance dynamics during the upgraded analyses of the LHCb and also SuperKEKB will play an important role in these studies.

\subsection{Step 8: $K^{+} \rightarrow \pi^{+} \nu \bar{\nu}, K_{L} \rightarrow \pi^{0} \nu \bar{\nu}$ and $K_{L} \rightarrow \mu^{+} \mu^{-}$}

\subsubsection{Preliminaries}

Among the top highlights of flavour physics in this decade will be the measurements of the branching ratios of two golden modes $K^{+} \rightarrow \pi^{+} \nu \bar{\nu}$ and $K_{L} \rightarrow \pi^{0} \nu \bar{\nu} . K^{+} \rightarrow$ $\pi^{+} \nu \bar{\nu}$ is $\mathrm{CP}$ conserving while $K_{L} \rightarrow \pi^{0} \nu \bar{\nu}$ is governed by $\mathrm{CP}$ violation. Both decays are dominated in the SM and many of its extensions by $Z$ penguin diagrams. It is well known that these decays are theoretically very clean and their branching ratios have been calculated within the SM including NNLO QCD corrections and electroweak

${ }^{5}$ The latest updates $[143,282$ show that the recent LHCb measurement of the CP asymmetry $A_{9} 247$ leads to a slightly stronger constraint on the imaginary part of $C_{10}^{\prime}:-1.5 \leq \Im\left(C_{10}^{\prime}\right) \leq 1.5$. 
corrections [153, 161, 283 285]. Moreover, extensive calculations of isospin breaking effects and non-perturbative effects have been done [286, 287]. Reviews of these two decays can be found in 288 291. In particular in 288 the status of NP contributions as of 2008 has been reviewed. A recent short review of NP signatures in Kaon decays can be found in [292].

Assuming that light neutrinos couple only to left-handed currents, the general short distance effective Hamiltonian describing both decays is given as follows

$$
\mathcal{H}_{\text {eff }}(\nu \nu)=g_{\mathrm{SM}}^{2} V_{t s}^{*} V_{t d} \times\left[X_{L}(K)\left(\bar{s} \gamma^{\mu} P_{L} d\right)+X_{R}(K)\left(\bar{s} \gamma^{\mu} P_{R} d\right)\right] \times\left(\bar{\nu} \gamma_{\nu} P_{L} \nu\right),
$$

where $g_{\mathrm{SM}}$ is defined in (91). We have suppressed the charm contribution that is represented by $P_{c}(X)$ below.

The resulting branching ratios for the two $K \rightarrow \pi \nu \bar{\nu}$ modes can be written generally as

$$
\begin{gathered}
\mathcal{B}\left(K^{+} \rightarrow \pi^{+} \nu \bar{\nu}\right)=\kappa_{+}\left[\left(\frac{\operatorname{Im} X_{\text {eff }}}{\lambda^{5}}\right)^{2}+\left(\frac{\operatorname{Re} X_{\text {eff }}}{\lambda^{5}}-P_{c}(X)\right)^{2}\right], \\
\mathcal{B}\left(K_{L} \rightarrow \pi^{0} \nu \bar{\nu}\right)=\kappa_{L}\left(\frac{\operatorname{Im} X_{\text {eff }}}{\lambda^{5}}\right)^{2},
\end{gathered}
$$

where 287

$$
\kappa_{+}=(5.36 \pm 0.026) \cdot 10^{-11}, \quad \kappa_{\mathrm{L}}=(2.31 \pm 0.01) \cdot 10^{-10}
$$

and 283 287].

$$
P_{c}(X)=0.42 \pm 0.03
$$

The short distance contributions are described by

$$
X_{\mathrm{eff}}=V_{t s}^{*} V_{t d}\left(X_{L}(K)+X_{R}(K)\right) \equiv V_{t s}^{*} V_{t d} X\left(x_{t}\right)\left(1+\xi e^{i \theta}\right) .
$$

Here

$$
X_{L}^{\mathrm{SM}}(K)=\eta_{X} X_{0}\left(x_{t}\right)=1.464 \pm 0.041,
$$

results within the SM from $Z$-penguin and box diagrams with

$$
X_{0}\left(x_{t}\right)=\frac{x_{t}}{8}\left[\frac{x_{t}+2}{x_{t}-1}+\frac{3 x_{t}-6}{\left(x_{t}-1\right)^{2}} \ln x_{t}\right],
$$

and $\eta_{X}=0.994$ for $m_{t}\left(m_{t}\right)$.

It should be remarked that with the definitions of electroweak parameters as in Table 1. in particular $\sin ^{2} \theta_{W}$, the electroweak corrections to $X_{L}^{\mathrm{SM}}(K)$ are totally negligible 161 and therefore are not exhibited here. To this end also $m_{t}\left(m_{t}\right)$, as discussed in the context of the $B_{s, d} \rightarrow \mu^{+} \mu^{-}$decays in Step 4, should be used. That is for $m_{t}$ only 

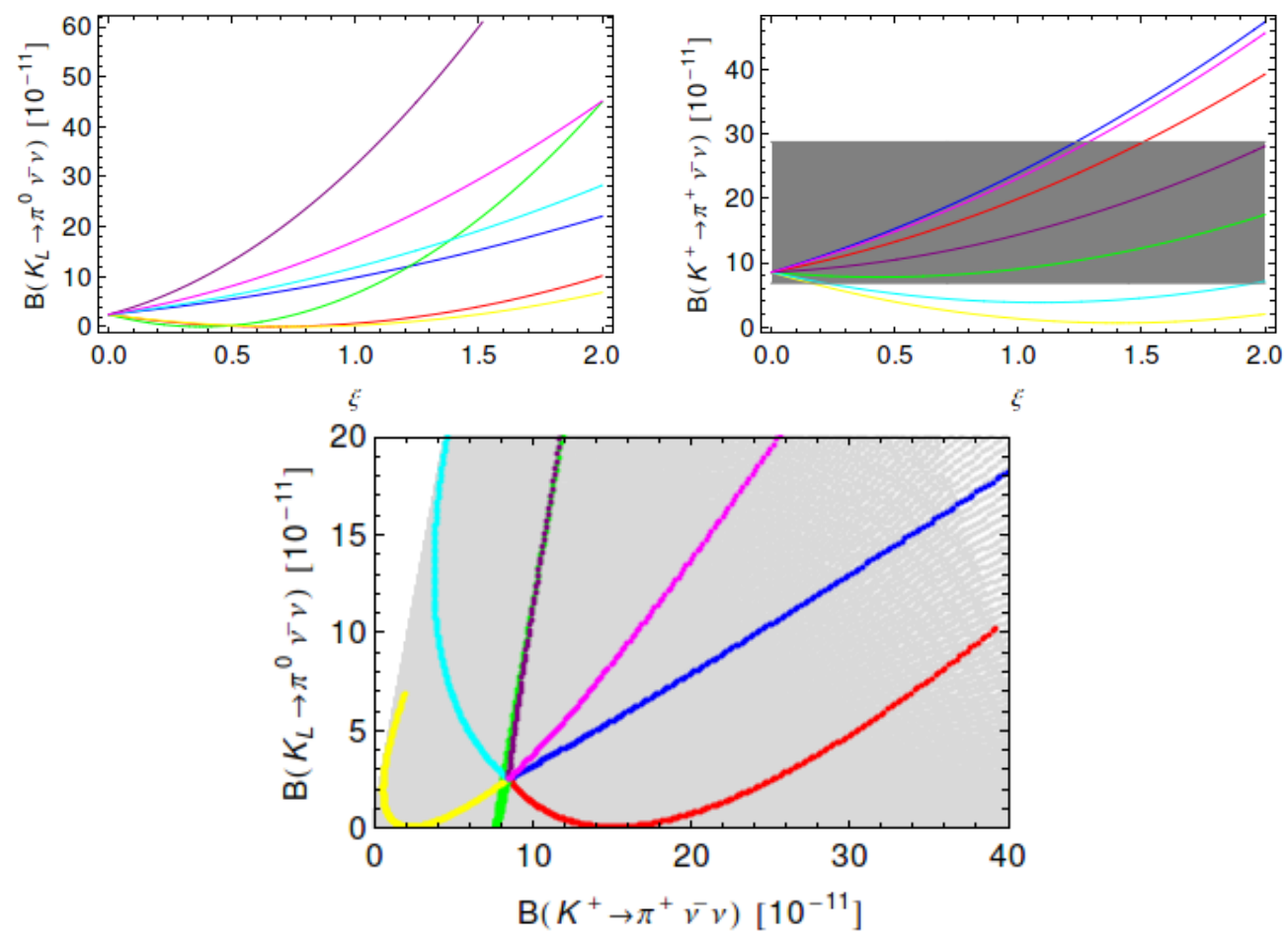

Figure 35: Top: $K_{L} \rightarrow \pi^{0} \nu \bar{\nu}$ and $K^{+} \rightarrow \pi^{+} \nu \bar{\nu}$ as a function of $\xi$ for $\theta=0$ (blue), 1 (red), 2 (green), 3 (yellow), 4 (cyan), 5 (purple), 6 (magenta). Down: $K_{L} \rightarrow \pi^{0} \nu \bar{\nu}$ vs. $K^{+} \rightarrow \pi^{+} \nu \bar{\nu}$ for $\xi \in[0,2]$ and $\theta \in[0,2 \pi]$ (light gray) and coloured $\theta$ as before.

QCD corrections are $\overline{\mathrm{MS}}$ renormalized, whereas $m_{t}$ is on-shell as far as electroweak corrections are concerned. See 157, 161 for more details.

In order to describe NP contributions we have introduced the two real parameters $\xi$ and $\theta$ that vanish in the SM. These formulae are in fact very general and apply to all extensions of the SM. The correlation between the two branching ratios depends generally on two variables $\xi$ and $\theta$ 288, 293,294] and measuring these branching ratios one day will allow to determine $\xi$ and $\theta$ and compare them with model expectations. We illustrate this in Fig. 35 .

Unfortunately on the basis of these two branching ratios it is not possible to find out how important the contributions of right-handed currents are as their effects are hidden in a single function $X_{\text {eff. }}$ In this sense the decays governed by $b \rightarrow s \nu \bar{\nu}$ transitions that we will discuss soon are superior. Indeed, in this case we have three branching ratios to our disposal and one is also sensitive to the direction of the spin of $K^{*}$.

Experimentally we have 295

$$
\mathcal{B}\left(K^{+} \rightarrow \pi^{+} \nu \bar{\nu}\right)_{\exp }=\left(17.3_{-10.5}^{+11.5}\right) \cdot 10^{-11},
$$

and the $90 \%$ C.L. upper bound 296

$$
\mathcal{B}\left(K_{L} \rightarrow \pi^{0} \nu \bar{\nu}\right) \exp \leq 2.6 \cdot 10^{-8} .
$$


The prospects for improved measurements of $\mathcal{B}\left(K^{+} \rightarrow \pi^{+} \nu \bar{\nu}\right)$ are very good. One should stress that already a measurement of this branching ratio with an accuracy of $10 \%$ will give us a very important insight into the physics at short distance scales. Indeed NA62 experiment at CERN aims at this precision and a new experiment at Fermilab (ORKA) should be able to reach the accuracy of $5 \%$ which would be truly fantastic. It will take longer in the case of $K_{L} \rightarrow \pi^{0} \nu \bar{\nu}$ but KOTO experiment at J-PARC should provide interesting results in this decade on this branching ratio. It should be emphasized that the combination of these two decays is particularly powerful in testing NP. The future prospects for experiments on $K$ decays, in particular $K^{+} \rightarrow \pi^{+} \nu \bar{\nu}$ and $K_{L} \rightarrow \pi^{0} \nu \bar{\nu}$ have been recently reviewed in 291,297.

The decays $K_{L} \rightarrow \pi^{0} \ell^{+} \ell^{-}$are not as theoretically clean as the $K \rightarrow \pi \nu \bar{\nu}$ channels and are less sensitive to NP contributions but they probe different operators beyond the SM and having accurate branching ratios for them would certainly be useful. Further details on this decay can be found in [298 303]. As there are no advanced plans to measure these branching ratios in this decade, we will not consider them in what follows. The most recent analysis of these decays within $Z^{\prime}$ models with further references can be found in 40.

On the other hand the decay $K_{L} \rightarrow \mu^{+} \mu^{-}$, even if subject to hadronic uncertainties, provides a useful constraint on the extensions of the SM. We will discuss this decay in this section as there are interesting correlations between this decay and $K^{+} \rightarrow \pi^{+} \nu \bar{\nu}$ which could help to distinguish between various NP scenarios.

For $K_{L} \rightarrow \mu^{+} \mu^{-}$the effective Hamiltonian, suppressing charm contribution and neglecting contributions from scalar operators that are suppressed by small $m_{d, s}$, reads

$$
\mathcal{H}_{\mathrm{eff}}(\mu \mu)=-g_{\mathrm{SM}}^{2} V_{t s}^{*} V_{t d} \times\left[Y_{L}(K)\left(\bar{s} \gamma^{\mu} P_{L} d\right)+Y_{R}(K)\left(\bar{s} \gamma^{\mu} P_{R} d\right)\right] \times\left(\bar{\mu} \gamma_{\nu} P_{L} \mu\right) .
$$

Only the so-called short distance (SD) part to a dispersive contribution to $K_{L} \rightarrow \mu^{+} \mu^{-}$ can be reliably calculated. We have then including charm contribution [293] $(\lambda=0.226)$

$$
\mathcal{B}\left(K_{L} \rightarrow \mu^{+} \mu^{-}\right)_{\mathrm{SD}}=2.08 \cdot 10^{-9}\left(\frac{\operatorname{Re} Y_{\text {eff }}^{K}}{\lambda^{5}}-\bar{P}_{c}(Y)\right)^{2}
$$

where at NNLO 304

$$
\bar{P}_{c}(Y) \equiv\left(1-\frac{\lambda^{2}}{2}\right) P_{c}(Y), \quad P_{c}(Y)=0.113 \pm 0.017 .
$$

The short distance contributions are described by

$$
Y_{\mathrm{eff}}^{K}=V_{t s}^{*} V_{t d}\left(Y_{L}(K)-Y_{R}(K)\right)
$$

with

$$
Y_{L}^{\mathrm{SM}}(K)=\eta_{Y} Y_{0}\left(x_{t}\right)
$$


already encountered in $B_{s, d} \rightarrow \mu^{+} \mu^{-}$decays and given in (124). We note the minus sign in front of $Y_{R}$ as opposed to $X_{R}$ in 202 that results from the fact that only the $\gamma_{\mu} \gamma_{5}$ part contributes.

The extraction of the short distance part from the data is subject to considerable uncertainties. The most recent estimate gives 305

$$
\mathcal{B}\left(K_{L} \rightarrow \mu^{+} \mu^{-}\right)_{\mathrm{SD}} \leq 2.5 \cdot 10^{-9},
$$

to be compared with $(0.8 \pm 0.1) \cdot 10^{-9}$ in the SM [304].

\subsubsection{Standard Model Results}

The branching ratios for $K^{+} \rightarrow \pi^{+} \nu \bar{\nu}$ and $K_{L} \rightarrow \pi^{0} \nu \bar{\nu}$ in the SM are given by

$$
\mathcal{B}\left(K^{+} \rightarrow \pi^{+} \nu \bar{\nu}\right)=\kappa_{+} \cdot\left[\left(\frac{\operatorname{Im} \lambda_{t}}{\lambda^{5}} X\left(x_{t}\right)\right)^{2}+\left(\frac{\operatorname{Re} \lambda_{t}}{\lambda^{5}} X\left(x_{t}\right)-P_{c}(X)\right)^{2}\right],
$$

and

$$
\mathcal{B}\left(K_{\mathrm{L}} \rightarrow \pi^{0} \nu \bar{\nu}\right)=\kappa_{\mathrm{L}} \cdot\left(\frac{\operatorname{Im} \lambda_{t}}{\lambda^{5}} X\left(x_{t}\right)\right)^{2} .
$$

The important feature of these expressions is that these two decays are described by the same real function $X\left(x_{t}\right)$. The present theoretical uncertainties in $\mathcal{B}\left(K^{+} \rightarrow \pi^{+} \nu \bar{\nu}\right)$ and $\mathcal{B}\left(K_{L} \rightarrow \pi^{0} \nu \bar{\nu}\right)$ are at the level of $2-3 \%$ and $1-2 \%$, respectively. Calculating the branching ratios for the central values of the parameters in Table 4, we find for $\left|V_{u b}\right|=0.0034$

$$
\mathcal{B}\left(K^{+} \rightarrow \pi^{+} \nu \bar{\nu}\right)_{\mathrm{SM}}=8.5 \cdot 10^{-11}, \quad \mathcal{B}\left(K_{L} \rightarrow \pi^{0} \nu \bar{\nu}\right)_{\mathrm{SM}}=2.5 \cdot 10^{-11},
$$

while for $\left|V_{u b}\right|=0.0040$ we find

$$
\mathcal{B}\left(K^{+} \rightarrow \pi^{+} \nu \bar{\nu}\right)_{\mathrm{SM}}=8.4 \cdot 10^{-11}, \quad \mathcal{B}\left(K_{L} \rightarrow \pi^{0} \nu \bar{\nu}\right)_{\mathrm{SM}}=3.4 \cdot 10^{-11} .
$$

We observe that whereas $\mathcal{B}\left(K^{+} \rightarrow \pi^{+} \nu \bar{\nu}\right)$ is rather insensitive to $\left|V_{u b}\right|, \mathcal{B}\left(K_{L} \rightarrow \pi^{0} \nu \bar{\nu}\right)$ increases with increasing $\left|V_{u b}\right|$. The main remaining uncertainty in these branching ratios comes from the $\left|V_{c b}\right|^{4}$ dependence and if the present value from tree-level decays is used, this uncertainty amounts to roughly $10 \%$. As we demonstrated in 45 this uncertainty within the SM can be decreased significantly with the help of $\varepsilon_{K}$, in particular when the angle $\gamma$ will be known from tree-level decays. Therefore, we expect that when the data from NA62 will be available, the total uncertainties in both branching ratios will be in the ballpark of $5 \%$.

These results should be compared with the experimental values given in (205) and (206). Certainly there is still a significant room left for NP contributions and we will now turn our attention to them in the context of simplest extensions of the SM. 


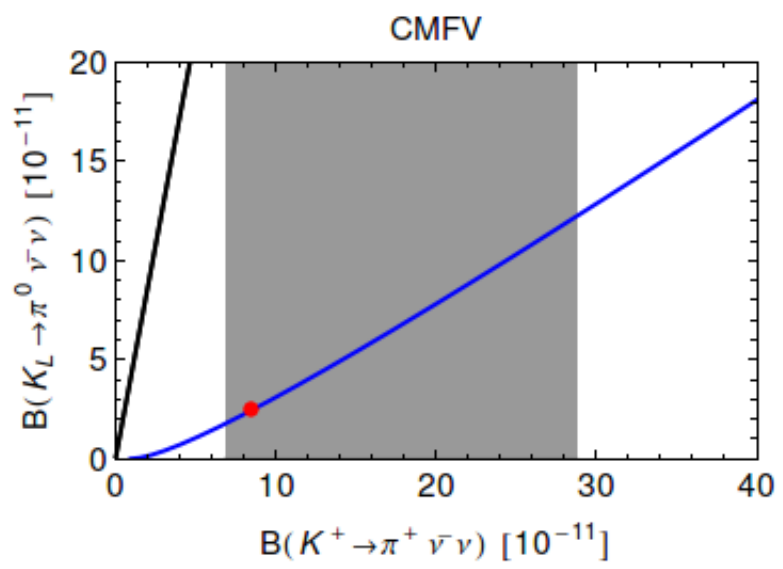

Figure 36: $\mathcal{B}\left(K_{L} \rightarrow \pi^{0} \nu \bar{\nu}\right)$ versus $\mathcal{B}\left(K^{+} \rightarrow \pi^{+} \nu \bar{\nu}\right)$ in $C M F V$. Red point: SM central value. Gray region: experimental range of $\mathcal{B}\left(K^{+} \rightarrow \pi^{+} \nu \bar{\nu}\right)$. The black line corresponds to the Grossman-Nir bound.

\subsubsection{CMFV}

In these models $K^{+} \rightarrow \pi^{+} \nu \bar{\nu}$ and $K_{L} \rightarrow \pi^{0} \nu \bar{\nu}$ are described by a single real function $X(v)$ implying a strong correlation between the two branching ratios as emphasized in [97]. We show this correlation in Fig. 36. Thus once the the branching ratio for $K^{+} \rightarrow \pi^{+} \nu \bar{\nu}$ will be measured with high precision by NA62 and later at Fermilab, we will know also precisely the corresponding branching ratio for $K_{L} \rightarrow \pi^{0} \nu \bar{\nu}$ that will be universal for the full class of CMFV models.

\subsection{4 $2 \mathrm{HDM}_{\overline{\mathrm{MFV}}}$}

In this class of models the dominant new contribution comes from charged Higgs $\left(H^{ \pm}\right)$ exchanges in $Z^{0}$-Penguin diagrams and box diagrams. While an explicit calculation with present input is missing we do not expect large NP contributions in this scenario.

\subsubsection{Tree-Level Gauge Boson Exchanges}

The contributions of tree-level exchanges to the branching ratios in question are known from various studies in $Z^{\prime}$ models. The new feature is the appearance of right-handed current contributions and the presence of new flavour violating interactions that can carry new CP-violating phases. A very detailed analysis of this simple NP scenario has been presented in [40] and we will summarize the most important results of this paper.

The branching ratios for the two $K \rightarrow \pi \nu \bar{\nu}$ modes are given by (198)-203) with 


$$
\begin{gathered}
X_{\mathrm{L}}(K)=\eta_{X} X_{0}\left(x_{t}\right)+\frac{\Delta_{L}^{\nu \bar{\nu}}\left(Z^{\prime}\right)}{g_{\mathrm{SM}}^{2} M_{Z^{\prime}}^{2}} \frac{\Delta_{L}^{s d}\left(Z^{\prime}\right)}{V_{t s}^{*} V_{t d}}, \\
X_{\mathrm{R}}(K)=\frac{\Delta_{L}^{\nu \bar{\nu}}\left(Z^{\prime}\right)}{g_{\mathrm{SM}}^{2} M_{Z^{\prime}}^{2}} \frac{\Delta_{R}^{s d}\left(Z^{\prime}\right)}{V_{t s}^{*} V_{t d}}
\end{gathered}
$$

As the new $\Delta_{L, R}^{s d}\left(Z^{\prime}\right)$ are complex numbers, these results are rather arbitrary. In a situation like this we have to look for other observables in the $K$ system that depend also on these couplings. Here the correlation of $K \rightarrow \pi \nu \bar{\nu}$ decays with $\varepsilon_{K}$ can give insights into the flavour structure of NP contributions and distinguish between models in which NP is dominated by left-handed currents or right-handed currents or both left-handed and right-handed currents with similar magnitude and phases [36]. In fact as pointed out in the latter paper a correlation between $\varepsilon_{K}$ and $K \rightarrow \pi \nu \bar{\nu}$ decays exists that is characteristic for all NP frameworks where the phase in $\Delta S=2$ amplitudes is the square of the CP-violating phase in $\Delta S=1 \mathrm{FCNC}$ amplitudes. This is for instance what happens in the Little Higgs model with $T$ parity [48]. The introduction of the three scenarios for $\Delta_{L, R}$ in Section 2 was motivated by this work and also by [35], where similar scenarios in the context of various supersymmetric flavour models have been analyzed. What is novel in our analysis of these scenarios is that in the presence of the dominance of NP contributions by tree-level exchanges, the correlations in question are particularly transparent.

We illustrate this in explicit terms now by considering the set

$$
\varepsilon_{K}, \quad K^{+} \rightarrow \pi^{+} \nu \bar{\nu}, \quad K_{L} \rightarrow \pi^{0} \nu \bar{\nu}, \quad K_{L} \rightarrow \mu^{+} \mu^{-}
$$

in the scenarios LHS, RHS and LRS for the $\Delta_{L, R}$ couplings in question.

The inclusion of $K_{L} \rightarrow \mu^{+} \mu^{-}$in this discussion leads to interesting results. Indeed now

$$
\begin{gathered}
Y_{L}(K)=Y\left(x_{t}\right)+\frac{\Delta_{A}^{\mu \bar{\mu}}\left(Z^{\prime}\right)}{g_{\mathrm{SM}}^{2} M_{Z^{\prime}}^{2}} \frac{\Delta_{L}^{s d}\left(Z^{\prime}\right)}{V_{t s}^{*} V_{t d}}, \\
Y_{R}(K)=\frac{\Delta_{A}^{\mu \bar{\mu}}\left(Z^{\prime}\right)}{g_{\mathrm{SM}}^{2} M_{Z^{\prime}}^{2}} \frac{\Delta_{R}^{s d}\left(Z^{\prime}\right)}{V_{t s}^{*} V_{t d}} .
\end{gathered}
$$

We note that up to the lepton couplings NP corrections are the same as in $X_{L, R}(K)$. However, very importantly the function $Y_{R}(K)$ enters with the opposite sign to $X_{R}(K)$ into the branching ratio for $K_{L} \rightarrow \mu^{+} \mu^{-}$so that effectively one has

$$
Y_{\mathrm{A}}(K)=\eta_{Y} Y_{0}\left(x_{t}\right)+\frac{\left[\Delta_{A}^{\mu \bar{\mu}}\left(Z^{\prime}\right)\right]}{M_{Z^{\prime}}^{2} g_{\mathrm{SM}}^{2}}\left[\frac{\Delta_{L}^{s d}\left(Z^{\prime}\right)-\Delta_{R}^{s d}\left(Z^{\prime}\right)}{V_{t s}^{\star} V_{t d}}\right] \equiv\left|Y_{A}(K)\right| e^{i \theta_{Y}^{K}} .
$$

The minus sign in front of $\Delta_{R}^{s d}\left(Z^{\prime}\right)$ implies an anti-correlation between $K^{+} \rightarrow \pi^{+} \nu \bar{\nu}$ and $K_{L} \rightarrow \mu^{+} \mu^{-}$branching ratios noticed already within the RSc scenario in [49]. 

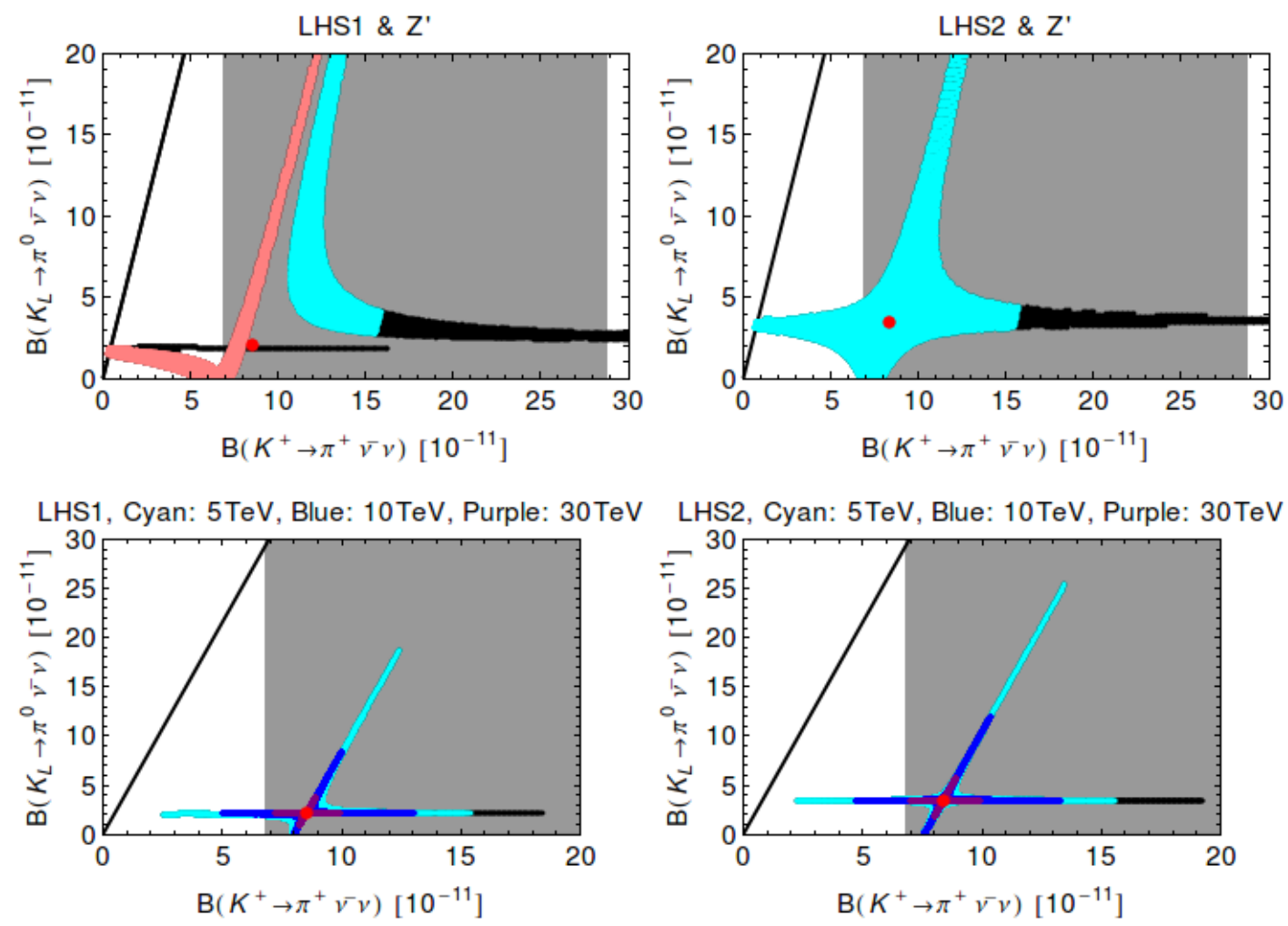

Figure 37: $\mathcal{B}\left(K_{L} \rightarrow \pi^{0} \nu \bar{\nu}\right)$ versus $\mathcal{B}\left(K^{+} \rightarrow \pi^{+} \nu \bar{\nu}\right)$ for $M_{Z^{\prime}}=1$ TeV (upper panels, $C_{1}$ : cyan, $C_{2}$ : pink.) and $M_{Z^{\prime}}=5 \mathrm{TeV}$ (cyan), $10 \mathrm{TeV}$ (blue) and $30 \mathrm{TeV}$ (purple) (lower panels) in LHS1 (left) and LHS2 (right). Black regions are excluded by the upper bound $\mathcal{B}\left(K_{L} \rightarrow \mu^{+} \mu^{-}\right) \leq 2.5 \cdot 10^{-9}$. Red point: SM central value. Gray region: experimental range of $\mathcal{B}\left(K^{+} \rightarrow \pi^{+} \nu \bar{\nu}\right)$. The black line corresponds to the Grossman-Nir bound.

We will now summarize the results obtained in [40], where the leptonic couplings have been chosen to be

$$
\Delta_{L}^{\nu \bar{\nu}}\left(Z^{\prime}\right)=\Delta_{A}^{\mu \bar{\mu}}\left(Z^{\prime}\right)=0.5
$$

to be compared with its SM value for $Z$ couplings 0.372 .

In our presentation of particular interest are the values of the $\delta_{12}$ phase in (97) 36

$$
\delta_{12}=n \frac{\pi}{2}, \quad n=0,1,2,3
$$

for which NP contributions to $\varepsilon_{K}$ vanish. As seen in Fig. 12 this is only allowed for scenario S2 for which SM agrees well with the data and NP contributions are not required. In this scenario $\tilde{s}_{12}$ can even vanish. In scenario $\mathrm{S} 1$, in which NP contributions are required to reproduce the data, $\tilde{s}_{12}$ is bounded from below and $\delta_{12}$ cannot satisfy (224). In the upper panels of Fig. 37 we show the correlation between the branching ratios for $K^{+} \rightarrow \pi^{+} \nu \bar{\nu}$ and $K_{L} \rightarrow \pi^{0} \nu \bar{\nu}$ in LHS1 and LHS2 for $M_{Z^{\prime}}=1 \mathrm{TeV}$ [40]. Since 
only vector currents occur we get the same result for RHS1 and RHS2. We observe the following pattern of deviations from the SM expectations:

- There are two branches in both scenarios. The difference between LHS1 and LHS2 originates from required NP contributions in LHS1 in order to agree with the data on $\varepsilon_{K}$ and the fact that in LHS1 there are two oases and only one in LHS2.

- The horizontal branch in both plots corresponds to $n=0,2$ in (224), for which NP contribution to $K \rightarrow \pi \nu \bar{\nu}$ is real and vanishes in the case of $K_{L} \rightarrow \pi^{0} \nu \bar{\nu}$.

- The second branch corresponds to $n=1,3$ in (224), for which NP contribution is purely imaginary. It is parallel to the Grossman-Nir (GN) bound [306] that is represented by the solid black line.

- The deviations from the SM are significantly larger than in the case of rare $B$ decays. This is a consequence of the weaker constraint from $\Delta S=2$ processes compared to $\Delta B=2$ and the fact that rare $K$ decays are stronger suppressed than rare $B$ decays within the SM. Yet as seen the largest values corresponding to black areas are ruled out through the correlation with $K_{L} \rightarrow \mu^{+} \mu^{-}$as discussed below.

- We observe that even at $M_{Z^{\prime}}=10 \mathrm{TeV}$ both branching ratios can still differ by much from SM predictions and for $M_{Z^{\prime}} \leq 20 \mathrm{TeV} \mathrm{NP}$ effects in these decays, in particular $K_{L} \rightarrow \pi^{0} \nu \bar{\nu}$, should be detectable in the flavour precision era.

Of particular interest is the correlation between $\mathcal{B}\left(K^{+} \rightarrow \pi^{+} \nu \bar{\nu}\right)$ and $\mathcal{B}\left(K_{L} \rightarrow \mu^{+} \mu^{-}\right)$ that we show in Fig. 38. In the case of LHS1 scenario a correlation analogous to this one is found in the LHT model [307] but due to fewer free parameters in $Z^{\prime}$ model this correlation depends whether oasis $C_{1}$ or $C_{2}$ is considered. The horizontal line in Fig. 38 corresponds this time to $n=1,3$ in (224), for which NP contribution is purely imaginary, while the other branches correspond to $n=0,2$ in (224), for which NP contribution to $K \rightarrow \pi \nu \bar{\nu}$ is real.

From Figs. 37 and 38 we obtain the following results:

- In the case of the dominance of real NP contributions we find for $M_{Z^{\prime}}=1 \mathrm{TeV}$

$$
\mathcal{B}\left(K^{+} \rightarrow \pi^{+} \nu \bar{\nu}\right) \leq 16 \cdot 10^{-11} .
$$

In this case $K_{L} \rightarrow \pi^{0} \nu \bar{\nu}$ is SM-like and $\mathcal{B}\left(K_{L} \rightarrow \mu^{+} \mu^{-}\right)$reaches the upper bound in (212).

- In the case of the dominance of imaginary NP contributions the bound on $\mathcal{B}\left(K_{L} \rightarrow\right.$ $\left.\mu^{+} \mu^{-}\right)$is ineffective and both $\mathcal{B}\left(K^{+} \rightarrow \pi^{+} \nu \bar{\nu}\right)$ and $\mathcal{B}\left(K_{L} \rightarrow \pi^{0} \nu \bar{\nu}\right)$ can be significantly larger than the SM predictions and $\mathcal{B}\left(K^{+} \rightarrow \pi^{+} \nu \bar{\nu}\right)$ can also be larger than its present experimental central value. We also find that for such large values the 

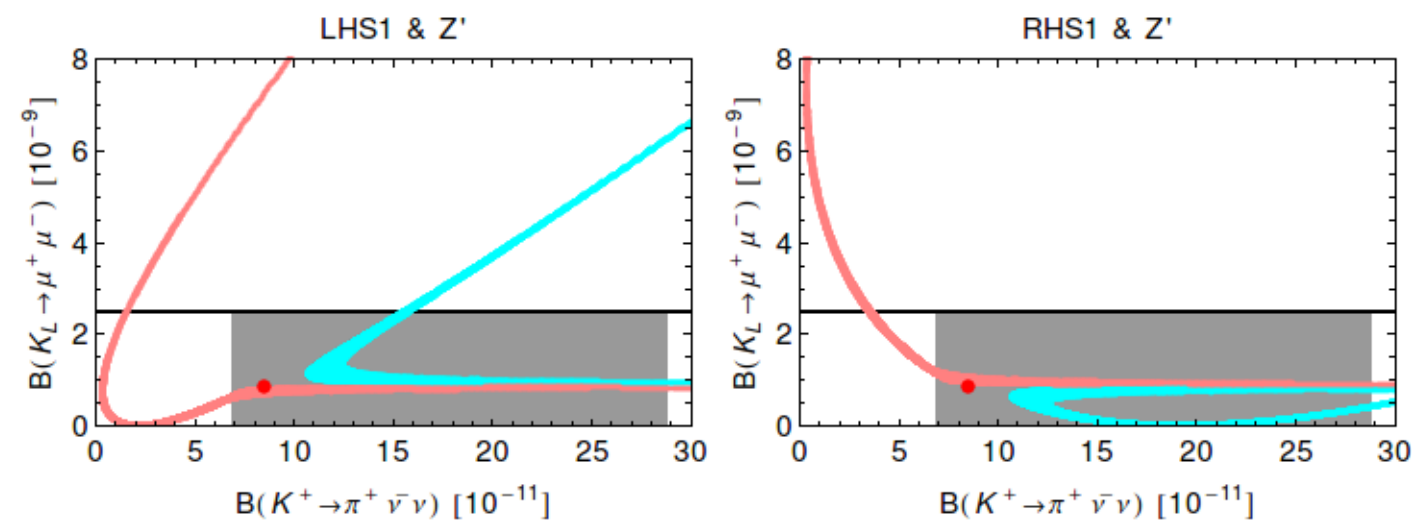

Figure 38: $\mathcal{B}\left(K_{L} \rightarrow \mu^{+} \mu^{-}\right)$versus $\mathcal{B}\left(K^{+} \rightarrow \pi^{+} \nu \bar{\nu}\right)$ for $M_{Z^{\prime}}=1$ TeV in LHS1 (left) and RHS1 (right). $C_{1}$ : cyan, $C_{2}$ : pink. Red point: SM central value. Gray region: experimental range of $\mathcal{B}\left(K^{+} \rightarrow \pi^{+} \nu \bar{\nu}\right)$ and horizontal black line: upper bound of $\mathcal{B}\left(K_{L} \rightarrow \mu^{+} \mu^{-}\right)$.

branching ratios are strongly correlated. Inspecting in the LHS2 scenario when the branch parallel to the GN bound leaves the grey region corresponding to the $1 \sigma$ region in 205 we find a rough upper bound

$$
\mathcal{B}\left(K_{L} \rightarrow \pi^{0} \nu \bar{\nu}\right) \leq 85 \cdot 10^{-11},
$$

which is much stronger than the present experimental upper bound in 206.

Finally, in the right panel of Fig. 38 we show the correlation between $\mathcal{B}\left(K^{+} \rightarrow \pi^{+} \nu \bar{\nu}\right)$ and $\mathcal{B}\left(K_{L} \rightarrow \mu^{+} \mu^{-}\right)$in the RHS1 scenario. Indeed the correlations in both oases differ from the ones in LHS1. This feature is known already from different studies, in particular in RSc scenario [49] and originates in the fact that while $K^{+} \rightarrow \pi^{+} \nu \bar{\nu}$ is sensitive to vector couplings, $K_{L} \rightarrow \mu^{+} \mu^{-}$is sensitive to the axial-vector couplings. We also note that in the case of the dominance of imaginary NP contributions corresponding to the horizontal line, $\mathcal{B}\left(K^{+} \rightarrow \pi^{+} \nu \bar{\nu}\right)$ and $\mathcal{B}\left(K_{L} \rightarrow \pi^{0} \nu \bar{\nu}\right)$ can be large. But otherwise $\mathcal{B}\left(K^{+} \rightarrow \pi^{+} \nu \bar{\nu}\right)$ is suppressed with respect to its $\mathrm{SM}$ value and $\mathcal{B}\left(K_{L} \rightarrow \pi^{0} \nu \bar{\nu}\right)$ is SM-like.

Finally we also discuss what happens if we exchange the $Z^{\prime}$ boson with the $Z^{0}$ boson with flavour violating couplings. Except for the LR scenario and in case of purely imaginary NP contributions these effects are bounded by $K_{L} \rightarrow \mu^{+} \mu^{-}$. In Fig. 39 we show our result for LHS2, RHS2 and LRS2 where the effects can be much larger than in the $Z^{\prime}$ case. 

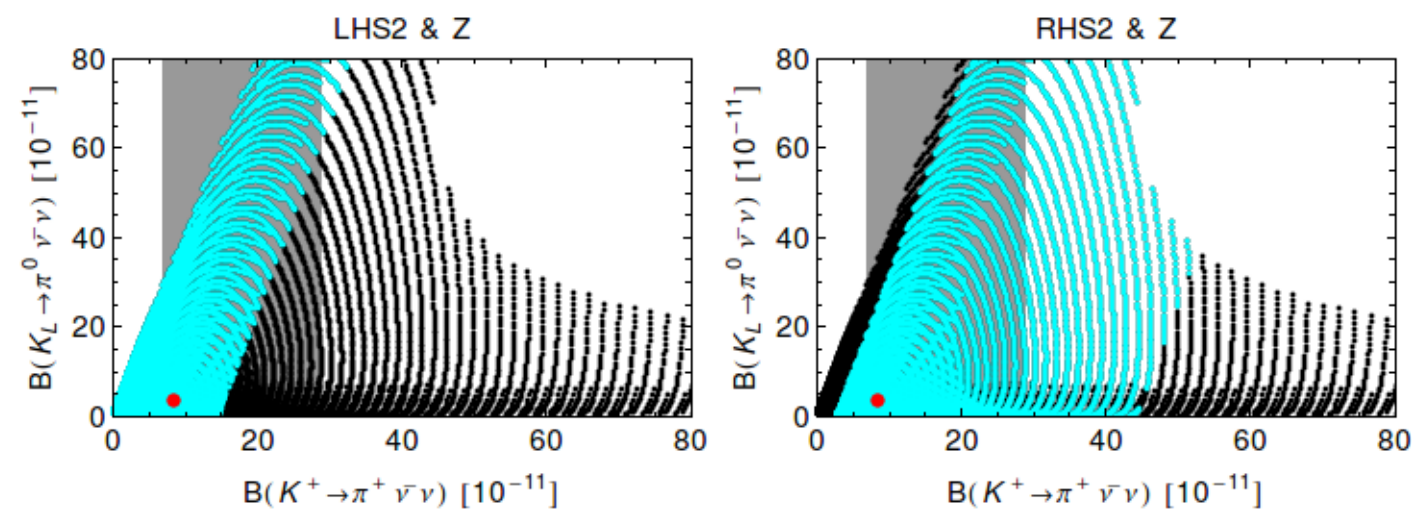

LRS2 \& Z

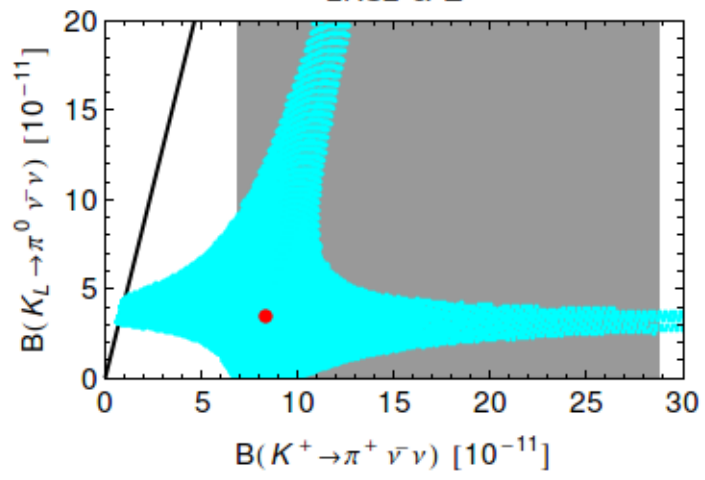

Figure 39: $\mathcal{B}\left(K_{L} \rightarrow \pi^{0} \nu \bar{\nu}\right)$ versus $\mathcal{B}\left(K^{+} \rightarrow \pi^{+} \nu \bar{\nu}\right)$ in LHS2, RHS2 and LRS2 for $Z^{0}$ exchange. Red point: $S M$ central value. Black regions are excluded by the upper bound $\mathcal{B}\left(K_{L} \rightarrow \mu^{+} \mu^{-}\right) \leq 2.5 \cdot 10^{-9}$. Gray region: experimental range of $\mathcal{B}\left(K^{+} \rightarrow \pi^{+} \nu \bar{\nu}\right)$.

\subsubsection{Tree-Level Scalar Exchanges}

If the masses of neutrinos are generated by the couplings to scalars than definitely the contributions of these scalars to decays with neutrinos in the final state are negligible. But if the masses of neutrinos are generated by a different mechanism than coupling to scalars, like in the case of the see-saw mechanism, it is not a priori obvious that such couplings in some NP scenarios could be measurable. Our working assumption in the present paper will be that this is not the case. Consequently NP effects of scalars in $K^{+} \rightarrow \pi^{+} \nu \bar{\nu}, K_{L} \rightarrow \pi^{0} \nu \bar{\nu}$ and $b \rightarrow s \nu \bar{\nu}$ transitions considered next will be assumed to be negligible in contrast to $Z^{\prime}$ models as we have just seen. As demonstrated in [44] scalar contributions to $K_{L} \rightarrow \mu^{+} \mu^{-}$and $K_{L} \rightarrow \pi^{0} \ell^{+} \ell^{-}$although in principle larger than for $K^{+} \rightarrow \pi^{+} \nu \bar{\nu}, K_{L} \rightarrow \pi^{0} \nu \bar{\nu}$ and $b \rightarrow s \nu \bar{\nu}$ transitions, are found to be small and we will not discuss them here. 


\subsection{Step 9: Rare B Decays $B \rightarrow X_{s} \nu \bar{\nu}, B \rightarrow K^{*} \nu \bar{\nu}$ and $B \rightarrow$ $K \nu \bar{\nu}$}

\subsubsection{Preliminaries}

The rare decays in question are among the important channels in $B$ physics as they allow a transparent study of $Z$ penguin and other electroweak penguin effects in NP scenarios in the absence of dipole operator contributions and Higgs (scalar) penguin contributions that are often more important than $Z$ contributions in $B \rightarrow K^{*} \ell^{+} \ell^{-}$and $B_{s} \rightarrow \ell^{+} \ell^{-}$decays $[308$ 310]. However, their measurements appear to be even harder than those of the rare $K$ decays just discussed. Yet, SuperKEKB should be able to measure them at a satisfactory level.

The inclusive decay $B \rightarrow X_{s} \nu \bar{\nu}$ is theoretically as clean as $K \rightarrow \pi \nu \bar{\nu}$ decays but the parametric uncertainties are a bit larger. The two exclusive channels are affected by form factor uncertainties but in the case of $B \rightarrow K^{*} \nu \bar{\nu}[310]$ and $B \rightarrow K \nu \bar{\nu}$ [311] significant progress has been made few years ago. In the latter paper this has been achieved by considering simultaneously also $B \rightarrow K \ell^{+} \ell^{-}$. Non-perturbative tree level contributions from $B^{+} \rightarrow \tau^{+} \nu$ to $B^{+} \rightarrow K^{+} \nu \bar{\nu}$ and $B^{+} \rightarrow K^{*+} \nu \bar{\nu}$ at the level of roughly $10 \%$ have been pointed out $[312$. Therefore the expressions in Eqs. (229)-(231) given below, as well as the SM results in (235), refer only to the short-distance contributions to these decays. The latter are obtained from the corresponding total rates subtracting the reducible long-distance effects pointed out in [312].

The general effective Hamiltonian including also right-handed current contributions that is used for the $B \rightarrow\left\{X_{s}, K, K^{*}\right\} \nu \bar{\nu}$ decays is given as follows

$$
\mathcal{H}_{\mathrm{eff}}=g_{\mathrm{SM}}^{2} V_{t s}^{*} V_{t b} \times\left[X_{L}\left(B_{s}\right)\left(\bar{s} \gamma^{\mu} P_{L} b\right)+X_{R}\left(B_{s}\right)\left(\bar{s} \gamma^{\mu} P_{R} b\right)\right] \times\left(\bar{\nu} \gamma_{\mu} P_{L} \nu\right)
$$

and has a very similar structure to the one for $K \rightarrow \pi \nu \bar{\nu}$ decays in (197). In particular

$$
X_{\mathrm{L}}^{\mathrm{SM}}\left(B_{s}\right)=X_{L}^{\mathrm{SM}}(K)
$$

with $X_{L}^{\mathrm{SM}}(K)$ given in (203). Moreover in models with minimal flavour violation (MFV) there is a striking correlation between the branching ratios for $K_{L} \rightarrow \pi^{0} \nu \bar{\nu}$ and $B \rightarrow X_{s} \nu \bar{\nu}$ as also there the same one-loop function $X(v)$ governs the two processes in question [97]. This relation is generally modified in models with non-MFV interactions, in particularly right-handed currents. As we will see below there are also correlations between $K_{L} \rightarrow \pi^{0} \nu \bar{\nu}, K^{+} \rightarrow \pi^{+} \nu \bar{\nu}$ and $B \rightarrow K^{*}(\rightarrow K \pi) \nu \bar{\nu}$ that are useful for the study of various NP scenarios.

The interesting feature of these three $b \rightarrow s \nu \bar{\nu}$ transitions, in particular when taken together, is their sensitivity to right-handed currents [308, 309] studied recently in [310]. Following the analysis of the latter paper, the branching ratios of the $B \rightarrow$ $\left\{X_{s}, K, K^{*}\right\} \nu \bar{\nu}$ modes in the presence of $\mathrm{RH}$ currents can be written as follows

$$
\mathcal{B}(B \rightarrow K \nu \bar{\nu})=\mathcal{B}(B \rightarrow K \nu \bar{\nu})_{\mathrm{SM}} \times[1-2 \eta] \epsilon^{2},
$$




$$
\begin{gathered}
\mathcal{B}\left(B \rightarrow K^{*} \nu \bar{\nu}\right)=\mathcal{B}\left(B \rightarrow K^{*} \nu \bar{\nu}\right)_{\mathrm{SM}} \times[1+1.31 \eta] \epsilon^{2}, \\
\mathcal{B}\left(B \rightarrow X_{s} \nu \bar{\nu}\right)=\mathcal{B}\left(B \rightarrow X_{s} \nu \bar{\nu}\right)_{\mathrm{SM}} \times[1+0.09 \eta] \epsilon^{2},
\end{gathered}
$$

where we have introduced the variables

$$
\epsilon^{2}=\frac{\left|X_{\mathrm{L}}\left(B_{s}\right)\right|^{2}+\left|X_{\mathrm{R}}\left(B_{s}\right)\right|^{2}}{\left|\eta_{X} X_{0}\left(x_{t}\right)\right|^{2}}, \quad \eta=\frac{-\operatorname{Re}\left(X_{\mathrm{L}}\left(B_{s}\right) X_{\mathrm{R}}^{*}\left(B_{s}\right)\right)}{\left|X_{\mathrm{L}}\left(B_{s}\right)\right|^{2}+\left|X_{\mathrm{R}}\left(B_{s}\right)\right|^{2}},
$$

with $X_{\mathrm{L}, \mathrm{R}}$ defined in (227).

We observe that the RH currents signaled here by a non-vanishing $\eta$ enter these three branching ratios in a different manner allowing an efficient search for the signals of these currents. Also the average of the $K^{*}$ longitudinal polarization fraction $F_{L}$ used in the studies of $B \rightarrow K^{*} \ell^{+} \ell^{-}$is a useful variable as it depends only on $\eta$ :

$$
\left\langle F_{L}\right\rangle=0.54 \frac{(1+2 \eta)}{(1+1.31 \eta)} .
$$

The experimental bounds 313 315 read

$$
\begin{gathered}
\mathcal{B}(B \rightarrow K \nu \bar{\nu})<1.4 \times 10^{-5}, \\
\mathcal{B}\left(B \rightarrow K^{*} \nu \bar{\nu}\right)<8.0 \times 10^{-5}, \\
\mathcal{B}\left(B \rightarrow X_{s} \nu \bar{\nu}\right)<6.4 \times 10^{-4} .
\end{gathered}
$$

\subsubsection{Standard Model Results}

In the absence of right-handed currents $\eta=0$ and all three decays are fully described by the function $X\left(x_{t}\right)$. The updated predictions for the SM branching ratios are $310 \sqrt[312]{312}$

$$
\begin{aligned}
\mathcal{B}(B \rightarrow K \nu \bar{\nu})_{\mathrm{SM}} & =(3.64 \pm 0.47) \times 10^{-6}, \\
\mathcal{B}\left(B \rightarrow K^{*} \nu \bar{\nu}\right)_{\mathrm{SM}} & =(7.2 \pm 1.1) \times 10^{-6}, \\
\mathcal{B}\left(B \rightarrow X_{s} \nu \bar{\nu}\right)_{\mathrm{SM}} & =(2.7 \pm 0.2) \times 10^{-5},
\end{aligned}
$$

\subsubsection{CMFV}

In this class of models all branching ratios are described as in Step 8 by the universal function $X(v)$

$$
X_{L}\left(B_{s}\right)=X(v), \quad X_{R}\left(B_{s}\right)=0
$$

and consequently they are strongly correlated. However, most characteristic for this class of models is the correlation between the $K \rightarrow \pi \nu \bar{\nu}$ branching ratios and the $b \rightarrow s \nu \bar{\nu}$ transitions considered here. This correlation is in particular stringent once the CKM parameters have been determined in tree-level decays. We show this in Fig. 40. 

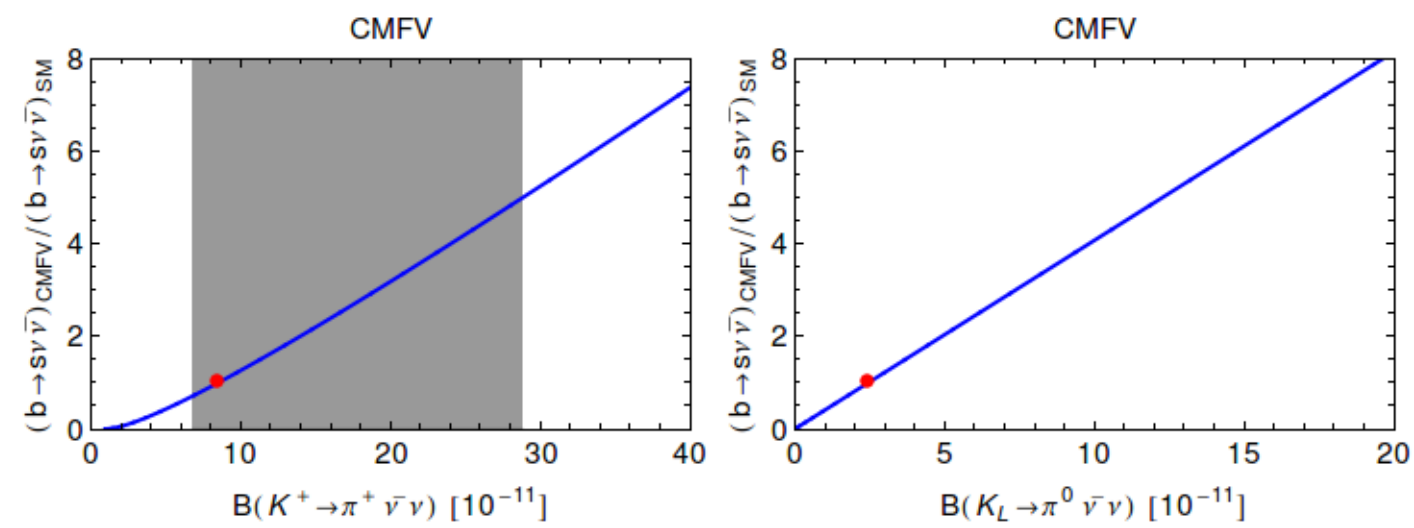

Figure 40: The ratio $\mathcal{B}\left(B \rightarrow K^{(*)} \nu \bar{\nu}\right)_{C M F V} / \mathcal{B}\left(B \rightarrow K^{(*)} \nu \bar{\nu}\right)_{S M}=\mathcal{B}(B \rightarrow$ $\left.X_{s} \nu \bar{\nu}\right)_{C M F V} / \mathcal{B}\left(B \rightarrow X_{s} \nu \bar{\nu}\right)_{S M}$ versus $K^{+} \rightarrow \pi^{+} \nu \bar{\nu}$ (left) and $K_{L} \rightarrow \pi^{0} \nu \bar{\nu}$ (right).

\subsection{4 $2 \mathrm{HDM}_{\overline{\mathrm{MFV}}}$}

To our knowledge, similarly to the case of $K \rightarrow \pi \nu \bar{\nu}$ decays, no detailed analysis of $b \rightarrow s \nu \bar{\nu}$ transitions exists in the literature. Yet because of tiny couplings of scalar particles to neutrinos such effects could only be relevant at one loop level with charged Higgs contributions at work. We expect these contributions to be small.

\subsubsection{Tree-Level Gauge Boson Exchanges}

Including the SM contribution in this case the couplings $X_{\mathrm{L}}$ and $X_{\mathrm{R}}$ are giving as follows

$$
\begin{gathered}
X_{\mathrm{L}}\left(B_{q}\right)=\eta_{X} X_{0}\left(x_{t}\right)+\left[\frac{\Delta_{L}^{\nu \nu}\left(Z^{\prime}\right)}{M_{Z^{\prime}}^{2} g_{\mathrm{SM}}^{2}}\right] \frac{\Delta_{L}^{q b}\left(Z^{\prime}\right)}{V_{t q}^{*} V_{t b}}, \\
X_{\mathrm{R}}\left(B_{q}\right)=\left[\frac{\Delta_{L}^{\nu \nu}\left(Z^{\prime}\right)}{M_{Z^{\prime}}^{2} g_{\mathrm{SM}}^{2}}\right] \frac{\Delta_{R}^{q b}\left(Z^{\prime}\right)}{V_{t q}^{*} V_{t b}},
\end{gathered}
$$

A detailed analysis of these decays has been performed in [40]. We summarize here the most important results of this analysis.

In Fig. 41 (left) we show $\mathcal{B}\left(B \rightarrow X_{s} \nu \bar{\nu}\right)$ vs $\mathcal{B}\left(B_{s} \rightarrow \mu^{+} \mu^{-}\right)$in LHS1 scenario. This correlation is valid in any oasis due to the assumed equal sign of the leptonic couplings in (223), although, as seen in the plot, the size of NP contribution may depend on the oasis considered. Significant NP effects are still possible and suppression of $\mathcal{B}\left(B_{s} \rightarrow \mu^{+} \mu^{-}\right)$ below the SM value will also imply the suppression of $\mathcal{B}\left(B \rightarrow X_{s} \nu \bar{\nu}\right)$. If the future data will disagree with this pattern, the rescue could come from the flip of the signs in $\nu \bar{\nu}$ or $\mu^{+} \mu^{-}$couplings provided this is allowed by leptonic decays of $Z^{\prime}$. As seen on the right of Fig. 41 additional information can come from the correlation between $\mathcal{B}\left(B \rightarrow X_{s} \nu \bar{\nu}\right)$ vs $S_{\psi \phi}$. 

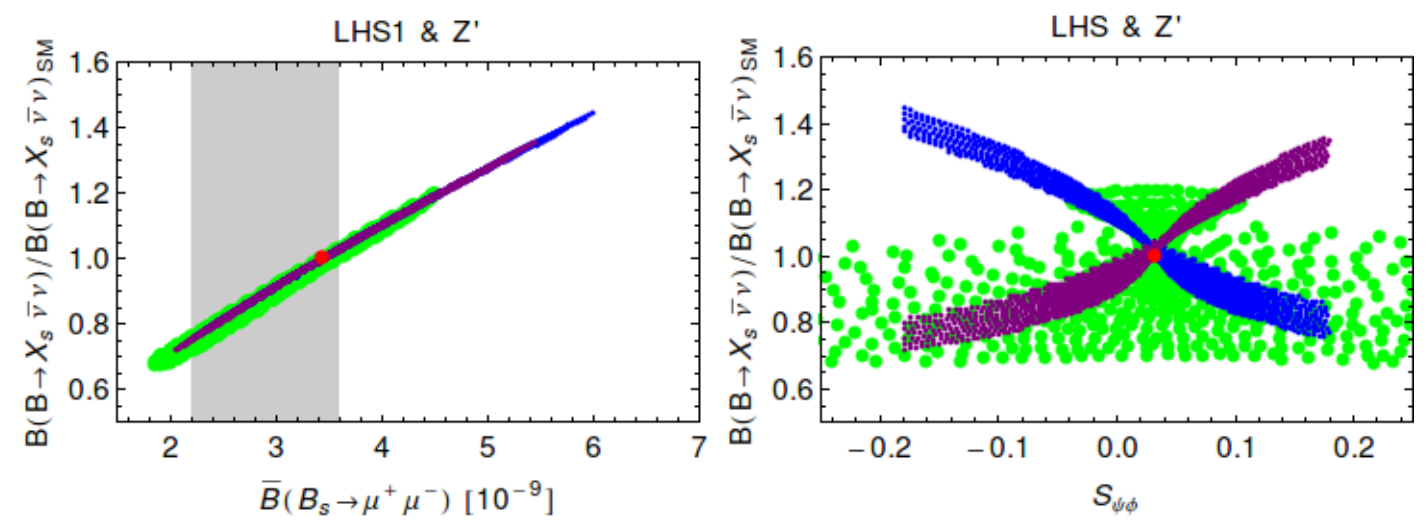

Figure 41: $\mathcal{B}\left(B \rightarrow X_{s} \nu \bar{\nu}\right)$ versus $\mathcal{B}\left(B_{s} \rightarrow \mu^{+} \mu^{-}\right)$(left) and $\mathcal{B}\left(B \rightarrow X_{s} \nu \bar{\nu}\right)$ versus $S_{\psi \phi}$ (right) in LHS1 for $M_{Z^{\prime}}=1 \mathrm{TeV}$. The green points indicate the regions that are compatible with $b \rightarrow s \ell^{+} \ell^{-}$constraints.

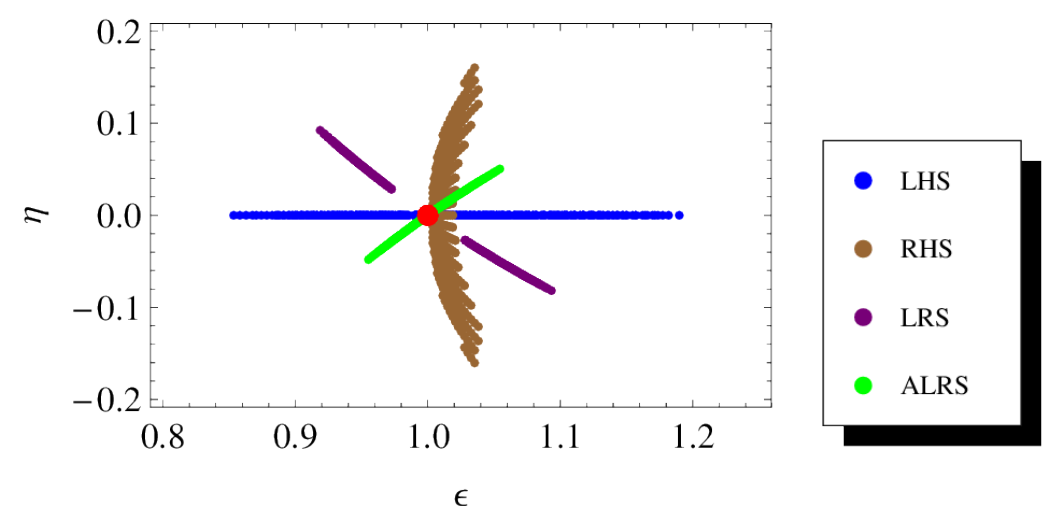

Figure 42: $\eta$ versus $\epsilon$ for scenario LHS1, RHS1, LRS1 and ALRS1.

As already emphasized above the decays in question are sensitive to the presence of right-handed currents. This is best seen in Fig. 42 where we show the results for all four scenarios considered by us in the $\epsilon-\eta$ plane. Indeed a future determination of $\epsilon$ and $\eta$ will tell us whether the nature chooses one of the scenario considered by us or a linear combination of them.

As $b \rightarrow s \ell^{+} \ell^{-}$transitions have large impact on the allowed size of right-handed currents we show two examples of it in Figs. 43 and 44. 

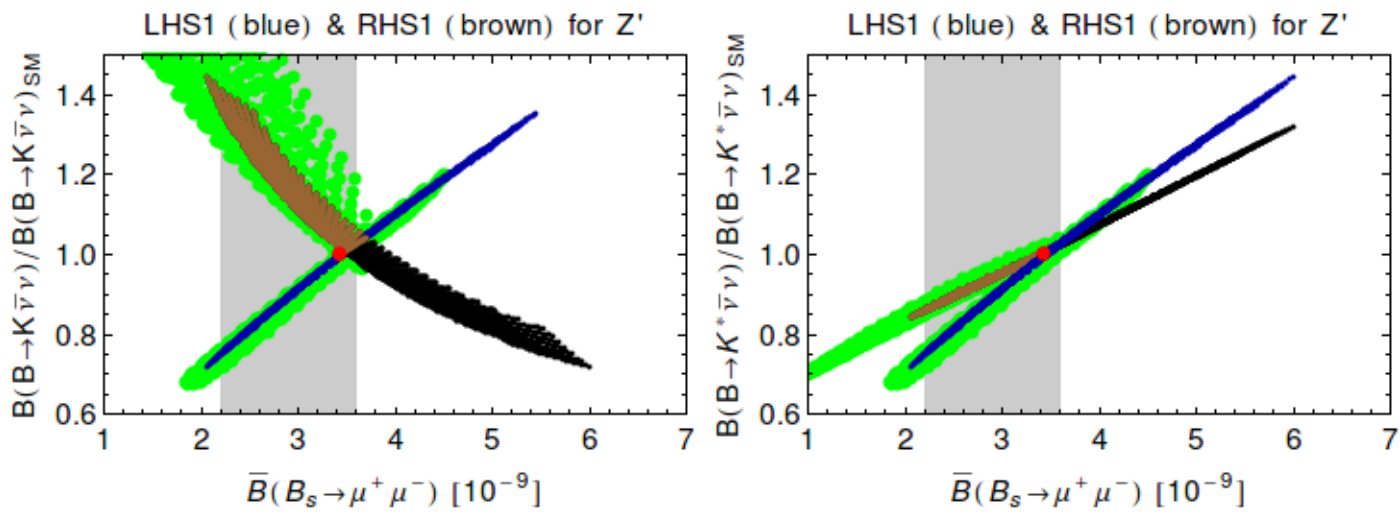

Figure 43: $\mathcal{B}(B \rightarrow K \nu \bar{\nu})$ versus $\mathcal{B}\left(B_{s} \rightarrow \mu^{+} \mu^{-}\right)$(left) and $\mathcal{B}\left(B \rightarrow K^{\star} \nu \bar{\nu}\right)$ versus $\mathcal{B}\left(B_{s} \rightarrow \mu^{+} \mu^{-}\right)$(right) for $M_{Z^{\prime}}=1$ TeV in LHS1 (blue for both oases $A_{1,3}$ ) and RHS1 (brown for both oases $\left.A_{1,3}\right)$ ). The green points indicate the regions that are compatible with $b \rightarrow s \ell^{+} \ell^{-}$constraints. Black points in RHS show the excluded area due to $b \rightarrow s \ell^{+} \ell^{-}$transitions explicitly. Gray region: exp $1 \sigma$ range $\overline{\mathcal{B}}\left(B_{s} \rightarrow \mu^{+} \mu^{-}\right)=$ $(2.9 \pm 0.7) \cdot 10^{-9}$. Red point: SM central value.
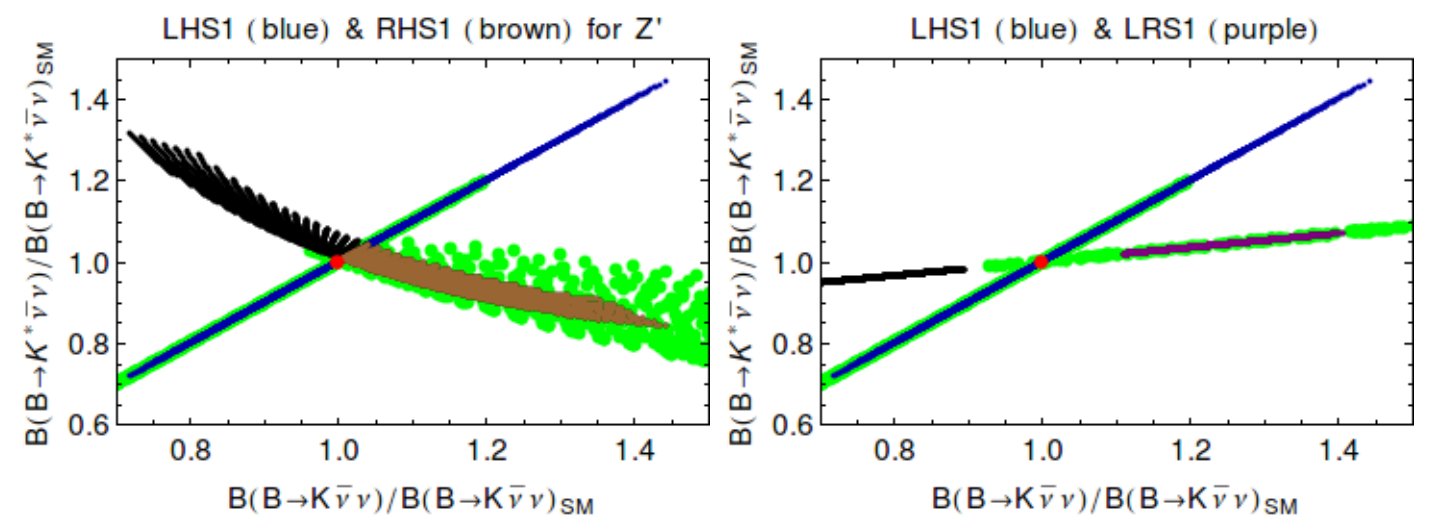

Figure 44: $\mathcal{B}\left(B \rightarrow K^{\star} \nu \bar{\nu}\right)$ versus $\mathcal{B}(B \rightarrow K \nu \bar{\nu})$ for $M_{Z^{\prime}}=1$ TeV in LHS1 (blue for both oases $A_{1,3}$ ), RHS1 (brown for both oases $A_{1,3}$ ) and LRS1 (purple for both oases $\left.A_{1,3}\right)$ ). The green points indicate the regions that are compatible with $b \rightarrow s \ell^{+} \ell^{-}$constraints. Black points in RHS show the excluded area due to $b \rightarrow s \ell^{+} \ell^{-}$transitions explicitly. Red point: SM central value.

\subsection{Step 10: The Ratio $\varepsilon^{\prime} / \varepsilon$}

\subsubsection{Preliminaries}

One of the important actors of the 1990s in flavour physics was the ratio $\varepsilon^{\prime} / \varepsilon$ that measures the size of the direct $\mathrm{CP}$ violation in $K_{L} \rightarrow \pi \pi$ relative to the indirect $\mathrm{CP}$ violation described by $\varepsilon_{K}$. In the $\mathrm{SM} \varepsilon^{\prime}$ is governed by QCD penguins but receives also 
an important destructively interfering contribution from electroweak penguins that is generally much more sensitive to NP than the QCD penguin contribution.

The big challenge in making predictions for $\varepsilon^{\prime} / \varepsilon$ within the SM and its extensions is the strong cancellation of QCD penguin contributions and electroweak penguin contributions to this ratio. In the SM QCD penguins give positive contributions, while the electroweak penguins negative ones. In order to obtain useful prediction for $\varepsilon^{\prime} / \varepsilon$ in the SM the precision on the corresponding hadronic parameters $B_{6}^{(1 / 2)}$ and $B_{8}^{(3 / 2)}$ should be at least $10 \%$. Recently significant progress has been made in the case of $B_{8}^{(3 / 2)}$ that is relevant for electroweak penguin contribution [316] but the calculation of $B_{6}^{(1 / 2)}$ is even more important. There are some hopes that also this parameter could be known with satisfactory precision in this decade [125, 317].

This would really be good, as the calculations of short distance contributions to this ratio (Wilson coefficients of QCD and electroweak penguin operators) within the SM have been known already for twenty years at the NLO level [318, 319] and present technology could extend them to the NNLO level if necessary. First steps in this direction have been done in 320,321 .

In the most studied extensions of the SM, the QCD penguin contributions are not modified significantly. On the other hand large NP contributions to electroweak penguins are possible. But they are often correlated with $K_{L} \rightarrow \pi^{0} \nu \bar{\nu}$ and $K^{+} \rightarrow \pi^{+} \nu \bar{\nu}$ decays so that considering $\varepsilon^{\prime} / \varepsilon$ and these two decays simultaneously useful constraints on model parameters can be derived, again subject to the uncertainties in $B_{6}^{(1 / 2)}$ and $B_{8}^{(3 / 2)}$.

The present experimental world average from NA48 322] and KTeV 323, 324,

$$
\varepsilon^{\prime} / \varepsilon=(16.6 \pm 2.3) \cdot 10^{-4},
$$

could have an important impact on several extensions of the SM discussed if $B_{6}^{(1 / 2)}$ and $B_{8}^{(3 / 2)}$ were known. An analysis of $\varepsilon^{\prime} / \varepsilon$ in the LHT model demonstrates this problem in explicit terms 325 . If one uses $B_{6}^{(1 / 2)}=B_{8}^{(3 / 2)}=1$ as obtained in the large $N$ approach [139,326], $\left(\varepsilon^{\prime} / \varepsilon\right)_{\mathrm{SM}}$ is in the ballpark of the experimental data although below it and sizable departures of $\mathcal{B}\left(K_{L} \rightarrow \pi^{0} \nu \bar{\nu}\right)$ from its SM value are not allowed. $K^{+} \rightarrow$ $\pi^{+} \nu \bar{\nu}$ being $\mathrm{CP}$ conserving and consequently not as strongly correlated with $\varepsilon^{\prime} / \varepsilon$ as $K_{L} \rightarrow \pi^{0} \nu \bar{\nu}$ could still be enhanced by $50 \%$. On the other hand if $B_{6}^{(1 / 2)}$ and $B_{8}^{(3 / 2)}$ are different from unity and $\left(\varepsilon^{\prime} / \varepsilon\right)_{\mathrm{SM}}$ disagrees with experiment, much more room for enhancements of rare $K$ decay branching ratios through NP contributions is available. See also new insight from the recent analysis in [119]. Reviews of $\varepsilon^{\prime} / \varepsilon$ can be found in 327331 .

\subsubsection{Basic Formula in the Standard Model}

In the SM ten operators pay the tribute to the $\varepsilon^{\prime} / \varepsilon$. These are 


\section{Current-Current:}

$$
Q_{1}=\left(\bar{s}_{\alpha} u_{\beta}\right)_{V-A}\left(\bar{u}_{\beta} d_{\alpha}\right)_{V-A} \quad Q_{2}=(\bar{s} u)_{V-A}(\bar{u} d)_{V-A}
$$

QCD-Penguins:

$$
\begin{aligned}
Q_{3} & =(\bar{s} d)_{V-A} \sum_{q=u, d, s, c, b}(\bar{q} q)_{V-A} & Q_{4} & =\left(\bar{s}_{\alpha} d_{\beta}\right)_{V-A} \sum_{q=u, d, s, c, b}\left(\bar{q}_{\beta} q_{\alpha}\right)_{V-A} \\
Q_{5} & =(\bar{s} d)_{V-A} \sum_{q=u, d, s, c, b}(\bar{q} q)_{V+A} & Q_{6} & =\left(\bar{s}_{\alpha} d_{\beta}\right)_{V-A} \sum_{q=u, d, s, c, b}\left(\bar{q}_{\beta} q_{\alpha}\right)_{V+A}
\end{aligned}
$$

\section{Electroweak Penguins:}

$$
\begin{aligned}
Q_{7} & =\frac{3}{2}(\bar{s} d)_{V-A} \sum_{q=u, d, s, c, b} e_{q}(\bar{q} q)_{V+A} & Q_{8} & =\frac{3}{2}\left(\bar{s}_{\alpha} d_{\beta}\right)_{V-A} \sum_{q=u, d, s, c, b} e_{q}\left(\bar{q}_{\beta} q_{\alpha}\right)_{V+A} \\
Q_{9} & =\frac{3}{2}(\bar{s} d)_{V-A} \sum_{q=u, d, s, c, b} e_{q}(\bar{q} q)_{V-A} & Q_{10} & =\frac{3}{2}\left(\bar{s}_{\alpha} d_{\beta}\right)_{V-A} \sum_{q=u, d, s, c, b} e_{q}\left(\bar{q}_{\beta} q_{\alpha}\right)_{V-A}
\end{aligned}
$$

Here, $\alpha, \beta$ denote colours and $e_{q}$ denotes the electric quark charges reflecting the electroweak origin of $Q_{7}, \ldots, Q_{10}$. Finally, $(\bar{s} d)_{V-A} \equiv \bar{s}_{\alpha} \gamma_{\mu}\left(1-\gamma_{5}\right) d_{\alpha}$.

The NLO renormalization group analysis of these operators is rather involved 318 . 319 but eventually one can derive an analytic formula in terms of the basic one-loop functions [328]. The most recent version of this formula is given as follows [119]

$$
\frac{\varepsilon^{\prime}}{\varepsilon}=a \operatorname{Im} \lambda_{t}^{(K)} \cdot F_{\varepsilon^{\prime}}\left(x_{t}\right)
$$

where $\lambda_{t}^{(K)}=V_{t d} V_{t s}^{*}, a=0.92 \pm 0.02$ and

$$
F_{\varepsilon^{\prime}}\left(x_{t}\right)=P_{0}+P_{X} X_{0}\left(x_{t}\right)+P_{Y} Y_{0}\left(x_{t}\right)+P_{Z} Z_{0}\left(x_{t}\right)+P_{E} E_{0}\left(x_{t}\right),
$$

with the first term dominated by QCD-penguin contributions, the next three terms by electroweak penguin contributions and the last term being totally negligible. The one-loop functions $X_{0}, Y_{0}$ and $Z_{0}$ can be found in (204), (124) and (125), respectively. The coefficients $P_{i}$ are given in terms of the non-perturbative parameters $R_{6}$ and $R_{8}$ defined in (249) as follows:

$$
P_{i}=r_{i}^{(0)}+r_{i}^{(6)} R_{6}+r_{i}^{(8)} R_{8} .
$$

The coefficients $r_{i}^{(0)}, r_{i}^{(6)}$ and $r_{i}^{(8)}$ comprise information on the Wilson-coefficient functions of the $\Delta S=1$ weak effective Hamiltonian at the NLO and their numerical values can be found in 119. These numerical values are chosen to satisfy the so-called $\Delta I=1 / 2$ rule and emphasize the dominant dependence on the hadronic matrix elements residing in the QCD-penguin operator $Q_{6}$ and the electroweak penguin operator 
$Q_{8}$. From Table 1 in [119] we find that for the central value of $\alpha_{s}\left(M_{Z}\right)=0.1185$ the largest are the coefficients $r_{0}^{(6)}$ and $r_{Z}^{(8)}$ representing QCD-penguin and electroweak penguin contributions, respectively:

$$
r_{0}^{(6)}=16.8, \quad r_{Z}^{(8)}=-12.6 .
$$

The fact that these coefficients are of the similar size but having opposite signs has been the problem since the end of 1980s when the electroweak penguin contribution increased in importance due to the large top-quark mass [332, 333].

The parameters $R_{6}$ and $R_{8}$ are directly related to the $B$-parameters $B_{6}^{(1 / 2)}$ and $B_{8}^{(3 / 2)}$ representing the hadronic matrix elements of $Q_{6}$ and $Q_{8}$, respectively. They are defined as

$$
R_{6} \equiv 1.13 B_{6}^{(1 / 2)}\left[\frac{114 \mathrm{MeV}}{m_{s}\left(m_{c}\right)+m_{d}\left(m_{c}\right)}\right]^{2}, \quad R_{8} \equiv 1.13 B_{8}^{(3 / 2)}\left[\frac{114 \mathrm{MeV}}{m_{s}\left(m_{c}\right)+m_{d}\left(m_{c}\right)}\right]^{2},
$$

where the factor 1.13 signals the decrease of the value of $m_{s}$ since the analysis in 328 has been done.

A detailed analysis of $\varepsilon^{\prime} / \varepsilon$ is clearly beyond this review and we would like to make only a few statements.

In [328] it has been found that with $R_{8}=1.0 \pm 0.2$ as obtained at that time from lattice QCD, the data could be reproduced within the SM for $R_{6}=1.23 \pm 0.16$. While in 2003 this value would correspond to $B_{6}^{(1 / 2)}=1.23$, the change in the value of $m_{s}$ would imply $B_{6}^{(1 / 2)}=1.05$, very close to the large $N$ value. Now the most recent evaluation of $B_{8}^{(3 / 2)}$ from lattice QCD [316,334, 335] finds $B_{8}^{(3 / 2)} \approx 0.65$ and thereby implying that $R_{8} \approx 0.8$.

A very recent analysis of $\varepsilon^{\prime} / \varepsilon$ in the SM [119] which uses this lattice result finds indeed that for $B_{6}^{(1 / 2)}=1.0$ the agreement of the SM with the data is good although parametric uncertainties, in particular due to $\left|V_{u b}\right|$ and $\left|V_{c b}\right|$, allow still for sizable NP contributions. Undoubtly we need sufficient precision on $B_{6}^{(1 / 2)}$ and these two CKM parameters in order to have a clear cut picture of $\varepsilon^{\prime} / \varepsilon$. We are looking forward to the improved values of $\left|V_{u b}\right|,\left|V_{c b}\right|, B_{6}^{(1 / 2)}$ and $B_{8}^{(3 / 2)}$ and expect that in the second half of this decade $\varepsilon^{\prime} / \varepsilon$ will become again an important actor in particle physics. The correlations with $K_{L} \rightarrow \pi^{0} \nu \bar{\nu}$ and $K^{+} \rightarrow \pi^{+} \nu \bar{\nu}$ reanalyzed recently in [119] should then help us to select favourite $\mathrm{NP}$ scenarios in particular if the experimental branching ratios for these decays will be known with sufficient accuracy.

\subsection{Step 11: Charm and Top Systems}

\subsubsection{Preliminaries}

Our review is dominated by mixing and decays in $K, B_{d}$ and $B_{s}$ meson systems. In the last two steps we want to emphasize that charm and top physics (this step) as well 
as lepton flavour violation, electric dipole moments and $(g-2)_{e, \mu}$ discussed in the next step play important roles in the search for new physics. Our discussion will be very brief but we hope that general statements and the selected references are still useful for non-experts.

\subsubsection{Charm}

The study of $D$ mesons allows to explore in a unique manner the physics of up-type quarks in FCNC processes. This involves $D^{0}-\bar{D}^{0}$ mixing, direct and mixing induced $\mathrm{CP}$-violation and rare decays of mesons. Excellent summary of the present experimental and theoretical status as well of the future prospects for this field can be found in chapter 4 of [22]. We cannot add anything new to the information given there but not working recently in this field we can provide a number of unbiased statements.

Charm decays have the problem that the intermediate scale of roughly $2 \mathrm{GeV}$ does not allow on the one hand to use methods like chiral perturbation theory or large $N$, that are useful for $K$ physics. On the other hand the methods as heavy quark effective theories are not as useful here as in the $B_{s, d}$ systems. Fortunately lattice simulation are mostly done around this scale so that the future of this field will definitely depend on the progress made by lattice QCD.

Due to the presence of down quarks in the loop diagrams governing FCNCs within the SM, GIM mechanism is very effective so that the short distance part of any SM contribution is strongly suppressed. Consequently the background to possible NP contributions from this part is significantly smaller than in the case of $K$ and $B_{s, d}$ meson systems. This is in particular the case of CP violation which is predicted to be tiny in $D$ meson system. Unfortunately large background to NP from hadronic effects make the study of NP effects in this system very challenging and even the originally large direct CP violation observed by LHCb [336] could not be uniquely attributed to the signs of NP. The recent update shows that the anomaly in question basically disappeared 337 but NP could still be hidden under hadronic uncertainties.

Yet, the situation could improve in the future and the large amount of theoretical work prompted by these initially exciting LHCb results will definitely be very useful when the data improve. It is impossible to review this work which is summarized in [22] and we will mention here only few papers that fit very well to the spirit of our review as they discuss correlations between $\mathrm{CP}$ violation in charm decays and other observables [338 340]. These correlations, as in the decays discussed by us in previous steps, depend on the model considered, so they may help to identify the NP at work. They do not only involve observables in charm system like rare decays $D^{0} \rightarrow \phi \gamma$ or $D^{0} \rightarrow \mu^{+} \mu^{-}$but also observables measured at high- $p_{T}$, such as $t \bar{t}$ asymmetries, another highlight from the LHC.

In this context one should mention correlations between $D$ and $K$, which could be used to constrain NP effects in $K$ system through the ones in charm and vice versa 341 , 
342. In particular the universality of $\mathrm{CP}$ violation in flavour-changing decay processes elaborated in [342] allows to predict direct correspondence between NP contributions to the direct $\mathrm{CP}$ violation in charm and $K_{L} \rightarrow \pi \pi$ represented by $\varepsilon^{\prime} / \varepsilon$. There is no question about that charm physics will play a significant role in the search for NP by constraining theoretical models and offering complementary information to the one available from $K$ and $B_{s, d}$ system. Yet, from the present perspective clear cut conclusions about the presence or absence of relevant NP contributions will be easier to reach by studying observables considered by us in previous steps.

\subsubsection{Top Quark}

The heaviest quark, the top quark, played already a dominant role in our review. It governs SM contributions to all observables discussed by us. The fact that the SM is doing well indicates that the structure of the CKM matrix with three hierarchical top quark couplings to lighter quarks

$$
\left|V_{t d}\right| \approx 8 \times 10^{-3}, \quad\left|V_{t s}\right| \approx 4 \times 10^{-2}, \quad\left|V_{t b}\right| \approx 1
$$

combined with the GIM mechanism represents the flavour properties of the top quark well. Yet, as the LHC became a top quark factory, properties of the top can be studied also directly, through its production and decay. In the latter case FCNC processes like $t \rightarrow c \gamma$ can be investigated. It is also believed that the top quark is closely related to various aspects of electroweak symmetry breaking and the problem of naturalness. Indeed, the top quark having the largest coupling to the Higgs field is the main reason for the severe fine tuning necessary to keep the Higgs mass close to the electroweak scale.

For these reasons we expect that the direct study of top physics, both flavour conserving and flavour violating will give us a profound insight into short distance dynamics, in particular as hadronic uncertainties at such short distance scales are much smaller than in decays of mesons. The observation of a large forward backward asymmetry in $t \bar{t}$ production at the Tevatron and the intensive theoretical studies aiming to explain this phenomenon have shown that this type of physics has great potential in constraining various extensions of the SM. As this material goes beyond the goals of our review we just wanted to emphasize that this is an important field in the search for NP. A useful collection of articles, which deal with top and flavour physics in the LHC era can be found in [343]. A detailed study of flavour sector with up vector-like quarks including correlations among various observables can be found in [169]. 


\subsection{Step 12: Lepton Flavour Violation, $(g-2)_{\mu, e}$ and EDMs}

\subsubsection{Preliminaries}

Our review deals dominantly with quark flavour violating processes. Yet in the search for NP an important role will also be played by

- Neutrino oscillations, neutrinoless double $\beta$ decay

- Charged lepton violation

- Anomalous magnetic moment of the muon $a_{\mu}=\frac{1}{2}(g-2)_{\mu}$

- Electric dipole moments of the neutron, atoms and leptons

In what follows we will only very briefly discuss these items. Selected reviews of these topics can be found in [7, 23, 344 346], where many references can be found. The study of correlations between LFV, $(g-2)_{\mu}$ and EDMs in supersymmetric flavour models and SUSY GUTS can be found in [35, 347, 349]. Analogous correlations in models with vector-like leptons have been presented in [350] and general expressions for these observables in terms of Wilson coefficients of dimension-six operators can be found in 351.

Concerning the first item, the observation of neutrino oscillations is a clear signal of physics beyond the SM and so far together with Dark Matter and the matter-antimatter asymmetry observed in our universe the only clear sign of NP. In order to accommodate neutrino masses one needs to extend the SM. The most straightforward way is to proceed in the same manner as for quark and charged lepton masses and just introduce three right-handed neutrinos that are singlets under the SM gauge group anyway. A Dirac mass term is then generated via the usual Higgs coupling $\bar{\nu}_{L} Y_{\nu} H \nu_{R}$. However then there is also the possibility for a Majorana mass term for the right-handed neutrinos since it is gauge invariant. One would need to introduce or postulate a further symmetry to forbid this term which is also already an extension of the SM. Furthermore this Majorana mass term introduces an additional scale $M_{R}$ and since it is not protected by any symmetry it could be rather high. Then the seesaw mechanism is at work and can generate light neutrino masses as observed in nature. Another possibility to get neutrino masses without right-handed neutrinos is the introduction of an additional Higgs-triplet field. Either way, the accommodation of neutrino masses requires an extension of the SM.

In the second and last point from above the interest in the related observables is based on the fact that they are suppressed within the SM to such a level that any observation of them would clearly signal physics beyond the SM. In this respect they differ profoundly from all processes discussed by us until now, which suffer from a large background coming from the SM and one needs precise theory and precise experiment to identify NP. Although $a_{e, \mu}$ are both flavour- and CP-conserving they also offer powerful probes to test NP. 


\subsubsection{Charged Lepton Flavour Violation}

The discovery of neutrino oscillations has shown that the individual lepton numbers are not conserved. However, no charged lepton flavour violating decays have been observed to date. In the SM enriched by light neutrino masses lepton-flavour violating decays $\ell_{j} \rightarrow \ell_{i} \gamma$ occur at unobservable small rates, because the transition amplitudes are suppressed by a factor of $\left(m_{\nu_{j}}^{2}-m_{\nu_{i}}^{2}\right) / M_{W}^{2}$. On the other hand in many extensions of the SM, like supersymmetric models, littlest Higgs model with T-parity (LHT) or the SM with sequential fourth generation (SM4) measurable in this decade branching ratios are predicted in particular when the masses of involved new particles are in the LHC reach. However, it should be stressed that in principle LFV can even be sensitive to energy scales as high as $1000 \mathrm{TeV}$. For a recent analysis within mini-split supersymmetry see 352 .

The most prominent role in the LFV studies play the decays

$$
\mu \rightarrow e \gamma, \quad \tau \rightarrow \mu \gamma, \quad \tau \rightarrow e \gamma
$$

but also the study of decays

$$
\mu^{-} \rightarrow e^{-} e^{+} e^{-}, \quad \tau^{-} \rightarrow \mu^{-} \mu^{+} \mu^{-}, \quad \tau^{-} \rightarrow e^{-} e^{+} e^{-}
$$

as well as $\mu-e$ conversion in nuclei offer in conjunction with $l_{i} \rightarrow l_{j} \gamma$ powerful tests of NP.

As our review is dominated by correlations let us just mention how a clear cut distinction between supersymmetric models, LHT model and SM4 is possible on the basis of these decays. While it is not possible to distinguish the LHT model from the supersymmetric models on the basis of $\mu \rightarrow e \gamma$ alone, it has been pointed out in [353] that such a distinction can be made by measuring any of the ratios $\mathcal{B}(\mu \rightarrow 3 e) / \mathcal{B}(\mu \rightarrow e \gamma)$, $\mathcal{B}(\tau \rightarrow 3 \mu) / \mathcal{B}(\tau \rightarrow \mu \gamma)$, etc. In supersymmetric models all these decays are governed by dipole operators so that these ratios are $\mathcal{O}(\alpha)$ 349, 354 359]. In the LHT model the LFV decays with three leptons in the final state are not governed by dipole operators but by $Z$-penguins and box diagrams and the ratios in question turn out to be by almost an order of magnitude larger than in supersymmetric models. Other analyses of LFV in the LHT model can be found in [360, 361] and in the MSSM in [349]. In the latter paper $(g-2)_{e}$ was used to probe lepton flavour violating couplings that are correlated with $\tau \rightarrow e \gamma$.

Similarly, as pointed out in [362] the pattern of the LFV branching ratios in the SM4 differs significantly from the one encountered in the MSSM, allowing to distinguish these two models with the help of LFV processes in a transparent manner. Also differences from the LHT model were identified.

A detailed analysis of LFV in various extensions of the SM is also motivated by the prospects in the measurements of LFV processes with much higher sensitivity than 
presently available. In particular the MEG experiment at PSI is already testing $\mathcal{B}(\mu \rightarrow$ $e \gamma$ ) at the level of $\mathcal{O}\left(10^{-13}\right)$. The current upper bound is [363]

$$
\mathcal{B}(\mu \rightarrow e \gamma) \leq 5.7 \cdot 10^{-13} .
$$

This bound puts also some GUT models under pressure as for example the model discussed in Sec. 6.5.6. An upgrade for MEG is also already approved [364] where they expect to improve the sensitivity down to $6 \cdot 10^{-14}$ after three years of running and there is an approved proposal at PSI to do $\mu \rightarrow$ eee [365]. The planned accuracy of SuperKEKB of $\mathcal{O}\left(10^{-8}\right)$ for $\tau \rightarrow \mu \gamma$ is also of great interest. This decay can also be studied at the LHC.

An improved upper bound on $\mu-e$ conversion in titanium will also be very important. In this context the dedicated J-PARC experiment PRISM/PRIME [366] should reach the sensitivity of $\mathcal{O}\left(10^{-18}\right)$, i. e. an improvement by six orders of magnitude relative to the present upper bound from SINDRUM-II at PSI [367]. Mu2e collaboration will measure $\mu-e$ conversion on aluminium to $6 \cdot 10^{-17}$ at $90 \% \overline{\mathrm{CL}}$ around 2020 [368] which is a factor of $10^{4}$ better than SINDRUM-II. Another improvement of a factor 10 is planed to be reached with Project $\mathrm{X}$ at Fermilab [27]. In [369] the model discriminating power of a combined phenomenological analysis of $\mu \rightarrow e \gamma$ and $\mu \rightarrow e$ conversion on different nuclei targets is discussed. They found that in most cases going from aluminuim to titanium is not very model-discriminating. A realistic discrimination among models requires a measure of $\mathcal{B}(\mu \rightarrow e, T i) / \mathcal{B}(\mu \rightarrow e, A l)$ at the level of $5 \%$ or better.

For further detailed review of LFV see $7,370,371]$. An experimenter's guide for charged $\mathrm{LFV}$ can be found in [346].

\subsubsection{Anomalous magnetic moments $(g-2)_{\mu, e}$}

The anomalous magnetic moment of the muon

$$
a_{\mu}=\frac{(g-2)_{\mu}}{2}
$$

provides an excellent test for physics beyond the SM. It can be extracted from the photon-muon vertex function $\Gamma^{\mu}\left(p^{\prime}, p\right)$

$$
\bar{u}\left(p^{\prime}\right) \Gamma^{\mu}\left(p^{\prime}, p\right) u(p)=\bar{u}\left(p^{\prime}\right)\left[\gamma^{\mu} F_{V}\left(q^{2}\right)+\left(p+p^{\prime}\right)^{\mu} F_{M}\left(q^{2}\right)\right] u(p),
$$

with

$$
a_{\mu}=-2 m_{\mu} F_{M}(0) .
$$

On the theory side $a_{\mu}$ receives four dominant contributions:

$$
a_{\mu}^{\mathrm{SM}}=a_{\mu}^{\mathrm{QED}}+a_{\mu}^{\mathrm{ew}}+a_{\mu}^{\gamma \gamma}+a_{\mu}^{\mathrm{hvp}} .
$$

While the QED [372 375] and electroweak contributions 344 376] to $a_{\mu}^{\text {SM }}$ are known very precisely and the light-by-light contribution $a_{\mu}^{\gamma \gamma}$ is currently known with an acceptable 
accuracy 377, 378, the theoretical uncertainty is dominated by the hadronic vacuum polarization. Review of the relevant calculations of all these contributions and related extensive analyses can be found in 344,379 .

According to the most recent analysis in [379], the very precise measurement of $a_{\mu}$ by the E821 experiment [380] in Brookhaven differs from its SM prediction by roughly $4.6 \sigma$ :

$$
a_{\mu}^{\exp }-a_{\mu}^{\mathrm{SM}}=(39.4 \pm 8.5) \times 10^{-10},
$$

where we added various errors discussed in [379] in quadrature.

Many models beyond the SM try to explain this discrepancy, especially supersymmetric models were very popular [381 387]. In SUSY the discrepancy could easily be accommodated for relatively light smuon masses and large $\tan \beta$. However so far no light SUSY particles have been discovered. Another approach was followed in [388] where the interplay of $(g-2)_{\mu}$ and a soft muon Yukawa coupling that is generated radiatively in the MSSM was studied. With the increased SUSY mass scale the explanation of $(g-2)_{\mu}$ anomaly becomes difficult 389.

Of course a new experiment would also be desirable. Fortunately, the $g-2$ ring at BNL has been disassembled and is on its way to Fermilab for a run around 2016. The overall error should go down by a factor of 2 . Thus if the central value will remain unchanged the discrepancy with the SM will increas to more than $8.0 \sigma$.

The anomalous magnetic moment of the muon $a_{\mu}$ is more sensitive to lepton flavour conserving NP than $a_{e}$ and consequently the latter was not as popular as $a_{\mu}$ in the last decade. However, as emphasized in [349], the fact that $a_{e}$ is very precisely measured and very precisely calculated within the SM it can also be used to probe NP, even if the theory agrees very well with experiment. Indeed, $a_{e}$ plays a central role in QED since its precise measurement provides the best source of $\alpha_{e}$ assuming the validity of QED [390]. Conversely, one can use a value of $\alpha_{\mathrm{em}}$ from a less precise measurement and insert it into the theory prediction for $a_{e}$ to probe NP. The most recent calculation yields $a_{e}=1159652182.79(7.71) \times 10^{-12}$ [391], where the largest uncertainty comes from the second-best measurement of $\alpha_{\mathrm{em}}$ which is $\alpha_{\mathrm{em}}^{-1}=137.03599884(91)$ from a Rubidium atom experiment 392]. Usually NP contributions to $a_{e}$ are small due to the smallness of the electron Yukawa coupling and the suppression of the NP scale. However, multiple flavour changes, resulting effectively in a lepton flavour conserving loop could be enhanced due to the $\tau$ Yukawa coupling [349].

\subsubsection{Electric Dipole Moments (EDMs)}

Even though the experimental sensitivities have improved a lot no EDM of a fundamental particle has been observed so far. Nevertheless EDM experiments have already put strong limits on NP models. A permanent EDM of a fundamental particle violates both $\mathrm{T}$ and $\mathrm{P}$, and thus - assuming CPT symmetry - is another way to measure $\mathrm{CP}$ violation. In the SM the only CP-violating phase of the CKM matrix enters quark 
EDMs first at three loop (two loop EW + one loop QCD) which results in negligibly small SM EDMs. Consequently EDMs are excellent probes of new CP violating phases of NP models, especially flavour blind phases, and of strong CP violation.

A recent review about EDMs can be found in [345] which updates the review in [393]. See also [394]. As discussed in [345] by naive dimension analysis EDMs probe a NP scale of several $\mathrm{TeV}$. This assumes order one $\mathrm{CP}$-violating phases $\phi_{\mathrm{CP}}$ for the electron EDM that arises at one loop order:

$$
d_{e} \approx e \frac{m_{e}}{\Lambda^{2}} \frac{\alpha_{e}}{4 \pi} \sin \phi_{\mathrm{CP}} \approx \frac{1}{2}\left(\frac{1 \mathrm{TeV}}{\Lambda}\right)^{2} \sin \phi_{\mathrm{CP}} \cdot 10^{-13} e \mathrm{fm} .
$$

Recently, the upper bound on $d_{e}$ has been improved by an order of magnitude with respect to the previous bound in [395] and reads [396]

$$
\left|d_{e}\right| \leq 8.7 \cdot 10^{-16} e \mathrm{fm} \text {. }
$$

This implies for the CP-violating phase $\left|\sin \phi_{\mathrm{CP}}\right| \lesssim\left(\frac{\Lambda}{6 \mathrm{TeV}}\right)^{2}$. The implications of this new bound on MFV have been investigated in [397] and other analyses are expected in the near future.

The scale of NP can be even higher for the neutron and ${ }^{199} \mathrm{Hg}$ EDMs as they are sensitive to the chromo-magnetic EDM which enters with a factor of $\alpha_{s}$ rather than the fine structure constant $\alpha_{e}$, pushing the sensitivity closer to $10 \mathrm{TeV}$. As one can see from (259) the sensitivity to the NP scale goes as $1 / \Lambda^{2}$, whereas in many other cases such as lepton flavour violation the sensitivity goes as $1 / \Lambda^{4}$. Future EDM measurements aim to improve their sensitivity by approximately two orders of magnitude which will then push the mass scale sensitivity into the (20-100) TeV range.

There are different sources for EDMs. For hadronic EDMs there is the $\theta$ term of QCD which is very much constrained due to the non-observation of permanent EDMs of the ${ }^{199} \mathrm{Hg}$ atom and neutron. Apart from the $\theta$ term, the SM CKM induced EDMs would be far smaller in magnitude than the next generation EDM sensitivities. Consequently, one does not need the same kind of refined hadronic structure computations as one often needs in flavour physics to interpret the EDM results in terms of NP. That being said, the hadronic matrix element problem remains a considerable challenge. At dimension six one encounters several different operators for the first generation fermions that could give rise to EDMs: pure gauge operators $\tilde{G} G G$, four-fermion operators (semi-leptonic and non-leptonic), gauge-higgs operators $\varphi^{\dagger} \varphi \tilde{G} G$ and gauge-higgs-fermion operators $\left(\bar{Q} T^{A} q_{R}\right) \varphi G$.

In experiments one often deals with composite systems and thus nuclear physics is important in determining the EDMs of neutral atoms. Nuclear structure can also provide an amplifier of atomic EDMs. In heavier neutral systems there is the shielding of the EDMs of constituents of one charge by those of the other (e.g. protons and electrons). The transmission of $\mathrm{CP}$ violation through a nucleus into an atom must 
overcome this shielding. Its effectiveness in doing so is expressed by a nuclear Schiff moment. In nuclei with asymmetric shapes Schiff moments can be enhanced by two or three orders of magnitude. For example an octupole deformed nuclei such as ${ }^{225}$ Ra give enhanced nuclear Schiff moments and, thus, an enhanced atomic EDMs in a diamagnetic system.

Flavour diagonal CP violating phases as needed for electroweak baryogenesis can be strongly constrained by EDMs. In the MSSM, for example, this requires rather heavy first and second generation sfermions but at the same time light electroweak gauginos below one $\mathrm{TeV}$ as well as a subset of the third generation sfermions (see [398] for details). However as can be deduced from the plots in [399] the improved bound on $d_{e}$ in (260) nearly excludes this possibility. While the bino-driven baryogenesis analyzed in [400] is still allowed by this new measurements, it further constraints this scenario.

A new an largely unexplored direction for electroweak baryogenesis is flavour nondiagonal CPV that would enter the $B$ or $D$ meson systems [401 403]. Flavour nondiagonal $\mathrm{CP}$ violation is far less susceptible to EDM constraints than flavour diagonal phases since it arises at multi-loop order. In the SM for example, it is a two-loop effect that involves the one-loop CP-violating penguin operator and a hadronic loop with two $\Delta S=1$ weak interactions.

Finally, let us quote recent studies of EDMs in 2HDM models with flavour blind phases [140,404] and supersymmetry [352] where further references to the rich literature can be found.

\section{Towards Selecting Successful Models}

\subsection{Preliminaries}

We have seen in previous sections that considering several theoretically clean observables in the context of various extensions of the SM there is a chance that we could identify new particles and new forces at very short distance scales that are outside the reach of the LHC. In fact this strategy is not new as most of elementary particles of the SM have been predicted to exist on the basis of low energy data well before their discovery ${ }^{6}$ Moreover, this has been achieved by not only the desire to understand the data but simultaneously with the goal to construct a fundamental theory of elementary matter and elementary interactions that is predictive and consistent with all physics principles we know. Yet, the present situation differs from the days when one started to discover first quarks in the following manner. Based on time and resources that were required to build the LHC it is rather unlikely that a machine probing directly $100-200 \mathrm{TeV}$ energy scales or short distance scales in the ballpark of a zeptometer $\left(10^{-21} \mathrm{~m}\right)$ will exist

\footnotetext{
${ }^{6}$ Although the non-vanishing neutrino masses came as a surprise and could be regarded as one of the first signs of NP beyond the SM.
} 
in the first half of this century. Rather a machine as an international linear collider with the energy of $1 \mathrm{TeV}$ will be build in order to study the details of physics up to this energy scale. Therefore, the search for new phenomena below $4 \times 10^{-20} \mathrm{~m}$, that is beyond the LHC, will be in the hands of flavour physics and very rare processes.

There is no question that the progress in the search for NP at the shortest distance scales will require an intensive collaboration of experimentalists and theorists. In this context there is the question whether top-down or bottom-up approach will turn out to be more efficient in reaching this goal. While bottom-up approach using exclusively effective theories with basically arbitrary coefficients of local operators allowed by symmetries of the SM can provide some insight in what is going on, we think that the top-down approach will eventually be more effective in the flavour precision era in identifying NP beyond the LHC reach. Yet, needless to say it would be extremely important to get some directions from direct discoveries of new phenomena at the LHC. This would in particular allow the correlations between high energy and low energy observables, which is only possible in a top-down approach.

Thus our basic strategy, as already exemplified on previous pages, is to look at different models and study different patterns of flavour violation in various theories through identification of correlations between various observables. The question then arises how to do it most efficiently and transparently.

In principle global fits of various observables in a given theory to the experimental data appears to be most straightforward. The success or failure of a given theory is then decided on the basis of $\chi^{2}$ or other statistical measures. This is clearly a legitimate approach and used almost exclusively in the literature. Yet, we think that in the first phase of the search for NP a more transparent approach could turn out to be more useful. This is what we will present next.

\subsection{DNA-Chart}

As reviewed in [17, 20] extensive studies of many models allowed to construct various classifications of NP contributions in the form of "DNA" tables [35] and flavour codes [17. The "DNA" tables in 35 had as a goal to indicate whether in a given theory a value of a given observable can differ by a large, moderate or only tiny amount from the prediction of the SM. The flavour codes [17] were more a description of a given model in terms of the presence or absence of left- or right-handed currents in it and the presence or absence of new CP phases, flavour violating and/or flavour conserving.

Certainly in both cases there is a room for improvements. In particular in the case of the "DNA" tables in [35] we know now that in most quark flavour observables considered there NP effects can be at most by a factor of 2 larger than the SM contributions. Exceptions are the cases in which some branching ratios or asymmetries vanish in the SM. But the particular weakness of this approach is the difficulty in depicting the correlations between various observables that could be characteristic for a given theory. 
Such correlations are much easier to show on a circle and in what follows we would like to formulate this new idea and illustrate it with few examples.

\section{Step 1}

We construct a chart showing different observables, typically a branching ratio for a given decay or an asymmetry, like CP-asymmetries $S_{\psi K_{S}}$ and $S_{\psi \phi}$ and quantities $\Delta M_{s}$, $\Delta M_{d}, \varepsilon_{K}$ and $\varepsilon^{\prime}$. The important point is to select the optimal set of observables which are simple enough so that definite predictions in a given theory can be made.

\section{Step 2}

In a given theory we calculate the selected observables and investigate whether a given observable is enhanced or suppressed relative to the SM prediction or is basically unchanged. What this means requires a measure, like one or two $\sigma$. In the case of asymmetries we will proceed in the same manner if its sign remains unchanged relative to the one in the SM but otherwise we define the change of its sign from + to - as a suppression and the change from - to + as an enhancement. For these three situations we will use the following colour coding:

$$
\text { enhancement }=\text { yellow }, \quad \text { no change }=\text { white } \quad \text { suppression }=\text { black }
$$

To this end the predictions within the SM have to be known precisely.

\section{Step 3}

It is only seldom that a given observable in a given theory is uniquely suppressed or enhanced but frequently two observables are correlated or uncorrelated, that is the enhancement of one observable implies uniquely an enhancement (correlation) or suppression (anti-correlation) of another observable. It can also happen that no change in the value of a given observable implies no change in another observable. There are of course other possibilities. The idea then is to connect in our DNA-chart a given pair of observables that are correlated with each other by a line. Absence of a line means that two given observables are uncorrelated. In order to distinguish the correlation from anti-correlation we will use the following colour coding for the lines in question:

$$
\text { correlation } \Leftrightarrow \text { blue }, \quad \text { anti }- \text { correlation } \Leftrightarrow \text { green }
$$

We will first make selection of the optimal observables that can be realistically measured in this decade and subsequently we will illustrate the DNA-chart on example of few simple models.

\subsection{Optimal Observables}

On the basis of our presentation in the previous sections we think that one should have first a closer look at the following observables. 


$$
\begin{gathered}
\varepsilon_{K}, \quad \Delta M_{s, d}, \quad S_{\psi K_{S}}, \quad S_{\psi \phi}, \\
K^{+} \rightarrow \pi^{+} \nu \bar{\nu}, \quad K_{L} \rightarrow \pi^{0} \nu \bar{\nu}, \quad \varepsilon^{\prime} / \varepsilon, \\
B_{s, d} \rightarrow \mu^{+} \mu^{-}, \quad B \rightarrow X_{s} \nu \bar{\nu}, \quad B \rightarrow K^{*}(K) \nu \bar{\nu}, \\
B \rightarrow X_{s} \gamma, \quad B^{+} \rightarrow \tau^{+} \nu_{\tau}, \quad B \rightarrow K^{*}(K) \mu^{+} \mu^{-},
\end{gathered}
$$

where in the latter case we mean theoretically clean angular observables. The remaining observables discussed by us will then serve as constraints on the model and if measured could also be chosen.

\subsection{Examples of DNA-Charts}

The first DNA-chart which one should in principle construct is the one dictated by experiment. This chart will have no correlation lines but will show where the SM disagrees with the data and comparing it with DNA-chart specific to a given theory will indicate which theories survived and which have been excluded. Unfortunately in view of significant uncertainties in some of the SM predictions and rather weak experimental bounds on most interesting branching ratios, such an experimental chart is rather boring at present as it is basically white. However, in the second half of this decade when LHC and other machines will provide new data and lattice calculations increase their precision it will possible to construct such an experimental DNA chart and we should hope that it will not be completely white.

Here we want to present four examples of DNA-charts. In Fig. 45 we show the DNAchart of CMFV and the corresponding chart for $U(2)^{3}$ models is shown in Fig. 46 . The DNA-charts representing models with left-handed and right-handed flavour violating couplings of $Z$ and $Z^{\prime}$ can be found in Fig. 47 .

The interested reader may check that these charts summarize compactly the correlations that we discussed in detail at various places in this review. In particular we observe the following features:

- When going from the DNA-chart of CMFV in Fig. 45 to the one for the $U(2)^{3}$ models in Fig. 46, the correlations between $K$ and $B_{s, d}$ systems are broken as the symmetry is reduced from $U(3)^{3}$ down to $U(2)^{3}$. The anti-correlation between $S_{\psi \phi}$ and $S_{\psi K_{S}}$ is just the one shown in Fig. 15. 
- As the decays $K^{+} \rightarrow \pi^{+} \nu \bar{\nu}, K_{L} \rightarrow \pi^{0} \nu \bar{\nu}$ and $B \rightarrow K \nu \bar{\nu}$ are only sensitive to the vector quark currents, they do not change when the couplings are changed from left-handed to right-handed ones. On the other hand the remaining three decays in Fig. 47 are sensitive to axial-vector couplings implying interchange of enhancements and suppressions when going from $L$ to $R$ and also change of correlations to anti-correlations between the latter three and the former three decays. Note that the correlation between $B_{s} \rightarrow \mu^{+} \mu^{-}$and $B \rightarrow K^{*} \mu^{+} \mu^{-}$does not change as both decays are sensitive only to axial-vector coupling.

- However, it should be remarked that in order to obtain the correlations or anticorrelations in LHS and RHS scenarios it was assumed that the signs of the left-handed couplings to neutrinos and the axial-vector couplings to muons are the same which does not have to be the case. If they are opposite the correlations between the decays with neutrinos and muons in the final state change to anticorrelations and vice versa.

- On the other hand due to $S U(2)_{L}$ symmetry the left-handed $Z^{\prime}$ couplings to muons and neutrinos are equal and this implies the relation

$$
\Delta_{L}^{\nu \bar{\nu}}\left(Z^{\prime}\right)=\frac{\Delta_{V}^{\mu \bar{\mu}}\left(Z^{\prime}\right)-\Delta_{A}^{\mu \bar{\mu}}\left(Z^{\prime}\right)}{2} .
$$

Therefore, once two of these couplings are determined the third follows uniquely without the freedom mentioned in the previous item.

- In the context of the DNA-charts in Fig. 47 , the correlations involving $K_{L} \rightarrow \pi^{0} \nu \bar{\nu}$ apply only if NP contributions carry some CP-phases. If this is not the case the branching ratio for $K_{L} \rightarrow \pi^{0} \nu \bar{\nu}$ will remain unchanged. This is evident from our discussion in Step 8 and the plots presented there.

In this context let as summarize the following important properties of the case of treelevel $Z^{\prime}$ and $Z$ exchanges when both LH and RH quark couplings are present which in addition are equal to each other (LRS scenario) or differ by sign (ALRS scenario):

- In LRS NP contributions to $B_{s, d} \rightarrow \mu^{+} \mu^{-}$vanish but not to $K_{L} \rightarrow \pi^{0} \nu \bar{\nu}$ and $K^{+} \rightarrow \pi^{+} \nu \bar{\nu}$.

- In ALRS NP contributions to $B_{s, d} \rightarrow \mu^{+} \mu^{-}$are non-vanishing and this also applies to $B_{d} \rightarrow K^{*} \mu^{+} \mu^{-}$as seen in the right panel of Fig. 34. On the other hand they vanish in the case of $K_{L} \rightarrow \pi^{0} \nu \bar{\nu}, K^{+} \rightarrow \pi^{+} \nu \bar{\nu}$ and $B_{d} \rightarrow K \mu^{+} \mu^{-}$ 


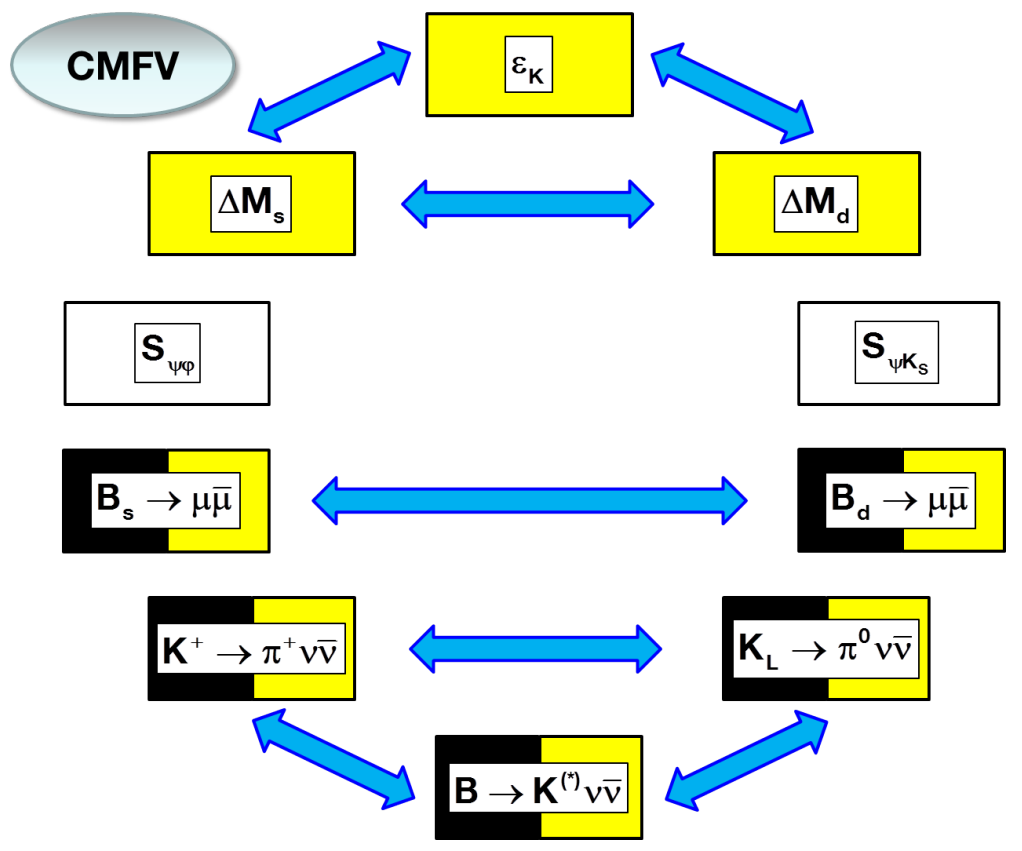

Figure 45: DNA-chart of CMFV models. Yellow means enhancement, black means suppression and white means no change. Blue arrows $\Leftrightarrow$ indicate correlation and green arrows $\Leftrightarrow$ indicate anti-correlation.

\subsection{Reviewing concrete models}

The realization of this strategy in the case of more complicated models is more challenging in view of many parameters involved, which often have to be determined beyond flavour physics. However we expect that when more data from the LHC and flavour machines around the world will be available it will be possible to be more concrete also in the case of these more complicated models. Two rather detailed reviews of various patterns of flavour violation in a number of favorite and less favorite extensions of the $\mathrm{SM}$ appeared in [17,20]. In view of the fact that no totally convincing signs of NP in flavour data has been observed since the appearance of the second review, there is no point in updating presently these reviews. Basically all these models can fit the present data by adjusting the parameters or increasing the masses of new particles. Therefore we only make a few remarks on some of these models and indicate in which section of [20] more details on a given model and related references to the original literature can be found.

\subsubsection{1 model}

A concrete example for $Z^{\prime}$ tree-level FCNC discussed in Sec. 3.7 and at various places in Sec. 5 is a model based on the gauge group $S U(3)_{C} \times S U(3)_{L} \times U(1)_{X}$, the so-called 


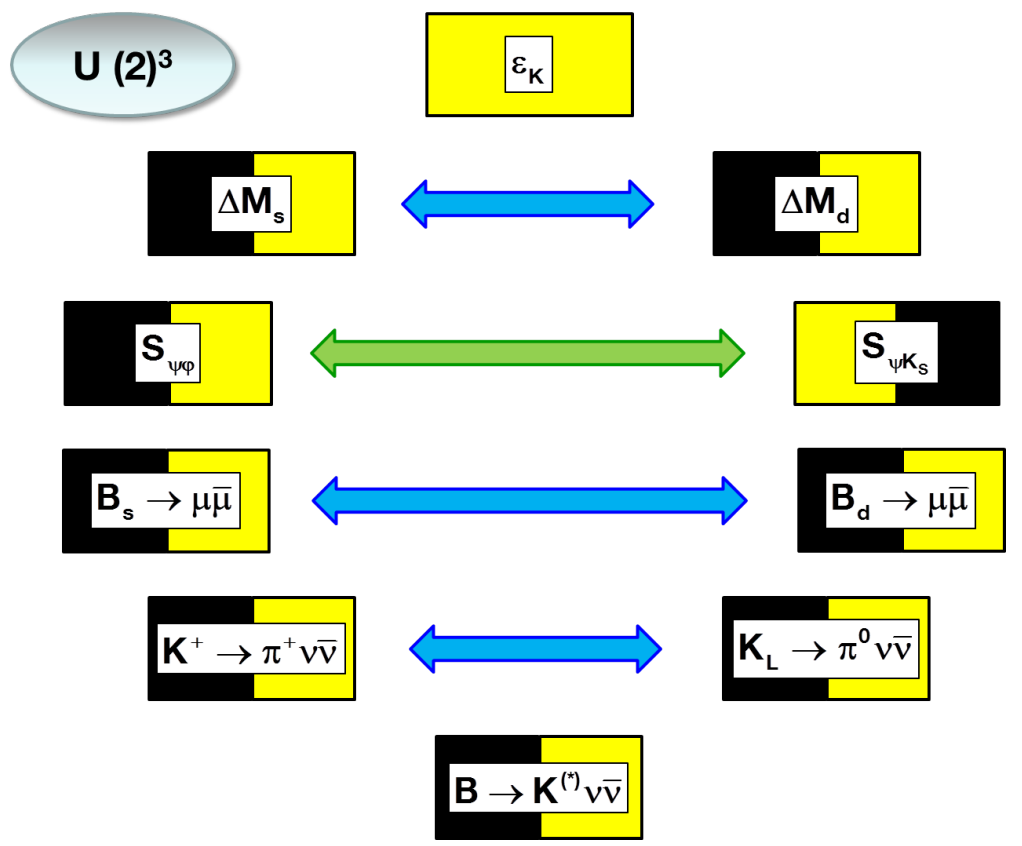

Figure 46: DNA-chart of $U(2)^{3}$ models. Yellow means enhancement, black means suppression and white means no change. Blue arrows $\Leftrightarrow$ indicate correlation and green arrows $\Leftrightarrow$ indicate anti-correlation.

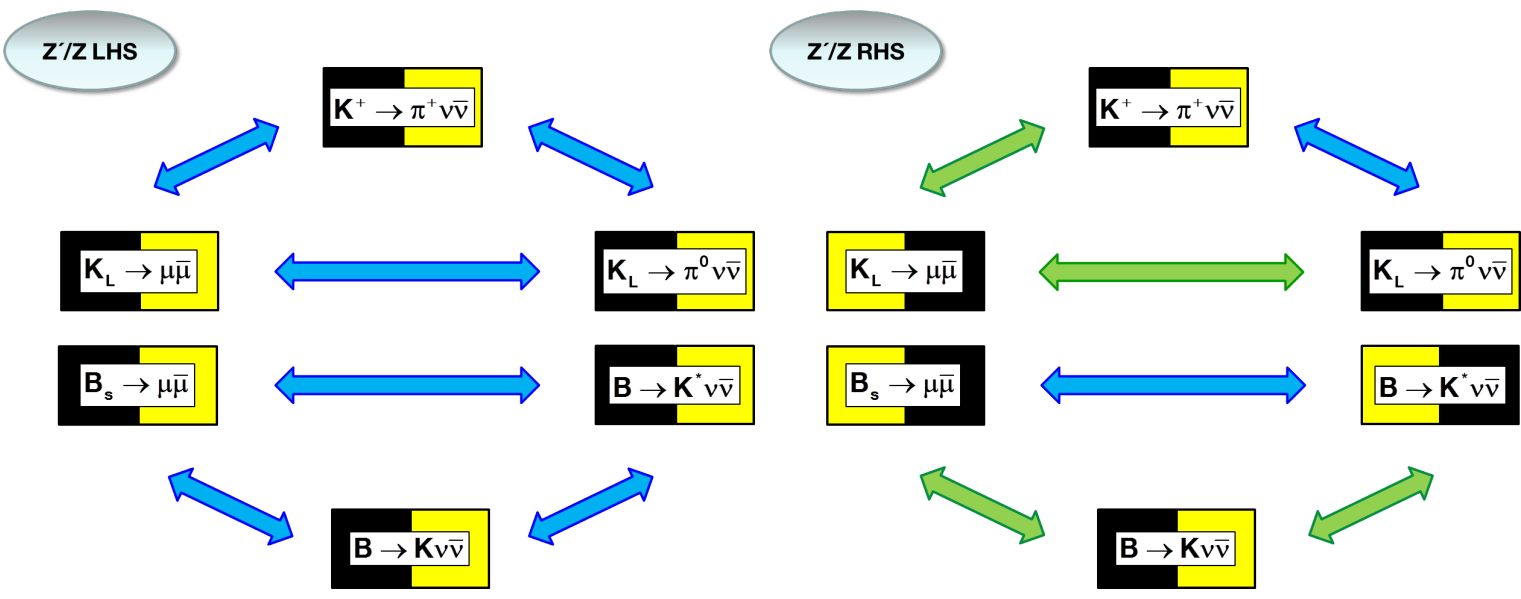

Figure 47: DNA-charts of $Z^{\prime}$ models with $L H$ and $R H$ currents. Yellow means enhancement, black means suppression and white means no change. Blue arrows $\Leftrightarrow$ indicate correlation and green arrows $\Leftrightarrow$ indicate anti-correlation.

331 model, originally developed in 405, 406. There are different versions of the 331 model characterized by a parameter $\beta$ that determines the particle content. In [41] we consider the $\beta=1 / \sqrt{3}$-model to be called $\overline{331}$ model. Since only left-handed currents are flavour violating and effects in $\varepsilon_{K}$ are rather small it favours inclusive $\left|V_{u b}\right|$ and thus 
belongs to LHS2. Furthermore also the lepton couplings are no longer arbitrary but come out automatically from the Lagrangian: $\Delta_{L}^{\nu \bar{\nu}}\left(Z^{\prime}\right)=0.14$ and $\Delta_{A}^{\mu \bar{\mu}}\left(Z^{\prime}\right)=-0.26$ for $\beta=1 / \sqrt{3}$. For the general $Z^{\prime}$ scenario we used $\Delta_{L}^{\nu \bar{\nu}}\left(Z^{\prime}\right)=0.5$ and $\Delta_{A}^{\mu \bar{\mu}}\left(Z^{\prime}\right)=0.5$.

In the breaking $S U(3)_{L} \times U(1)_{X} \rightarrow S U(2)_{L} \times U(1)_{Y}$ to the SM gauge group a new heavy neutral gauge boson $Z^{\prime}$ appears that mediates FCNC already at tree level. A nice theoretical feature is that from the requirement of anomaly cancellation and asymptotic freedom of QCD it follows that one needs $N=3$ generations. Anomaly cancellation is only possible if one generation (usually the $3^{\text {rd }}$ is chosen) is treated differently than the other two generations.

Further studies of the 331 model can be found in [407,408] where the lepton sector was analyzed in detail and in [409 411] where mixing of neutral mesons as well as a number of rare $K$ and $B_{d, s}$ decays have been considered. The decay $b \rightarrow s \gamma$ was considered in [412,413] and in [414] also neutral scalar contributions were included.

\section{Flavour structure}

The $\overline{331}$ model studied in [41] has the following fermion content: Left-handed fermions fit in (anti)triplets, while right-handed ones are singlets under $S U(3)_{L}$. In the quark sector, the first two generations fill the two upper components of a triplet, while the third one fills those of an anti-triplet; the third member of the quark (anti)triplet is a new heavy fermion:

$$
\begin{aligned}
& \left(\begin{array}{c}
e \\
-\nu_{e} \\
\nu_{e}^{c}
\end{array}\right)_{L},\left(\begin{array}{c}
\mu \\
-\nu_{\mu} \\
\nu_{\mu}^{c}
\end{array}\right)_{L},\left(\begin{array}{c}
\tau \\
-\nu_{\tau} \\
\nu_{\tau}^{c}
\end{array}\right)_{L}, \quad\left(\begin{array}{l}
u \\
d \\
D
\end{array}\right)_{L},\left(\begin{array}{c}
c \\
s \\
S
\end{array}\right),\left(\begin{array}{c}
b \\
-t \\
T
\end{array}\right)_{L} \\
& e_{R}, \mu_{R}, \tau_{R}, \quad u_{R}, d_{R}, c_{R}, s_{R}, t_{R}, b_{R}, \quad D_{R}, S_{R}, T_{R}
\end{aligned}
$$

We need the same number of triplets and anti-triplets due to anomaly cancellation. If one takes into account the three colours of the quarks we have six triplets and six anti-triplets with this choice. Neutral currents mediated by $Z^{\prime}$ are affected by the quark mixing because the $Z^{\prime}$ couplings are generation non-universal. However only left-handed quark currents are flavour-violating, thus we are left with LHS. Except for the $Z^{\prime}$ mass the tree-level FCNCs in $B_{d, s}$ and $K$ meson systems depend effectively on 2 angles and 2 phases $\tilde{s}_{23}, \tilde{s}_{13}, \delta_{1,2}$ such that the $B_{d}$ sector depends only on $\tilde{s}_{13}, \delta_{1}$ and the $B_{s}$ sector on $\tilde{s}_{23}, \delta_{2}$. Then in contrast to the general $Z^{\prime}$ models, discussed before, the NP parameters in $K$ sector are fixed. In particular CP violation is governed there by the phase difference $\delta_{2}-\delta_{1}$. In more general $Z^{\prime}$ models the $K$ sector is decoupled from $B_{d, s}$ sector.

Concerning phenomenology, it is more restrictive than the one in a general $Z^{\prime}$ model with left-handed couplings and it is of interest to investigate how the 331 models with arbitrary $\beta$ face the new data on $B_{s, d} \rightarrow \mu^{+} \mu^{-}$and $B_{d} \rightarrow K^{*}(K) \mu^{+} \mu^{-}$taking into account present constraints from $\Delta F=2$ observables, low energy precision measurements, LEP-II and the LHC data. Such an analysis has been performed in 47] and we 
summarize the main results of this paper where numerous correlations between various flavour observables can be found.

Studying the implications of these models for $\beta= \pm n / \sqrt{3}$ with $n=1,2,3$ we find that the case $\beta=-\sqrt{3}$ leading to Landau singularities for $M_{Z^{\prime}} \approx 4 \mathrm{TeV}$ can be ruled out when the present constraints on $Z^{\prime}$ couplings, in particular from LEP-II, are taken into account. For $n=1,2$ interesting results are found for $M_{Z^{\prime}}<4 \mathrm{TeV}$ with largest NP effects for $\beta<0$ in $B_{d} \rightarrow K^{*} \mu^{+} \mu^{-}$and the ones in $B_{s, d} \rightarrow \mu^{+} \mu^{-}$for $\beta>0$. As $\operatorname{Re}\left(C_{9}^{\mathrm{NP}}\right)$ can reach the values -0.8 and -0.4 for $n=2$ and $n=1$, respectively the $B_{d} \rightarrow K^{*} \mu^{+} \mu^{-}$ anomalies can be softened with the size depending on $\Delta M_{s} /\left(\Delta M_{s}\right)_{\mathrm{SM}}$ and the CPasymmetry $S_{\psi \phi}$. A correlation between $\operatorname{Re}\left(C_{9}^{\mathrm{NP}}\right)$ and $\overline{\mathcal{B}}\left(B_{s} \rightarrow \mu^{+} \mu^{-}\right)$, identified for $\beta<0$, implies for negative $\operatorname{Re}\left(C_{9}^{\mathrm{NP}}\right)$ uniquely suppression of $\overline{\mathcal{B}}\left(B_{s} \rightarrow \mu^{+} \mu^{-}\right)$relative to its SM value which is favoured by the data. In turn also $S_{\psi \phi}<S_{\psi \phi}^{\mathrm{SM}}$ is favoured with $S_{\psi \phi}$ having dominantly opposite sign to $S_{\psi \phi}^{\mathrm{SM}}$ and closer to its central experimental value. Another triple correlation is the one between $\operatorname{Re}\left(C_{9}^{\mathrm{NP}}\right), \overline{\mathcal{B}}\left(B_{s} \rightarrow \mu^{+} \mu^{-}\right)$and $\mathcal{B}\left(B_{d} \rightarrow K \mu^{+} \mu^{-}\right)$. NP effects in $b \rightarrow s \nu \bar{\nu}$ transitions, $K^{+} \rightarrow \pi^{+} \nu \bar{\nu}$ and $K_{L} \rightarrow \pi^{0} \nu \bar{\nu}$ turn out to be small.

We find also that the absence of $B_{d} \rightarrow K^{*} \mu^{+} \mu^{-}$anomalies in the future data and confirmation of the suppression of $\overline{\mathcal{B}}\left(B_{s} \rightarrow \mu^{+} \mu^{-}\right)$relative to its SM value would favour the $\overline{331}$ model $(\beta=1 / \sqrt{3})$ summarized in detail above and $M_{Z^{\prime}} \approx 3 \mathrm{TeV}$. Assuming lepton universality, we find an upper bound $\left|C_{9}^{\mathrm{NP}}\right| \leq 1.1(1.4)$ from LEP-II data for all $Z^{\prime}$ models with only left-handed flavour violating couplings to quarks when NP contributions to $\Delta M_{s}$ at the level of $10 \%(15 \%)$ are allowed.

Finally, we refer to a very recent analysis in 415 in which additional effects of $Z-Z^{\prime}$ mixing and resulting $Z$-mediated FCNCs have been investigated in detail. We find that these new contributions can indeed be neglected in the case of $\Delta F=2$ transitions and decays, like $B_{d} \rightarrow K^{*} \mu^{+} \mu^{-}$, where they are suppressed by the small vectorial $Z$ coupling to charged leptons. However, the contributions of tree-level $Z$ exchanges to decays sensitive to axial-vector couplings, like $B_{s, d} \rightarrow \mu^{+} \mu^{-}$and $B_{d} \rightarrow K \mu^{+} \mu^{-}$, and those with neutrinos in the final state, like $b \rightarrow s \nu \bar{\nu}$ transitions, $K^{+} \rightarrow \pi^{+} \nu \bar{\nu}$ and $K_{L} \rightarrow \pi^{0} \nu \bar{\nu}$ cannot be generally neglected with size of $Z$ contributions depending on $\beta, M_{Z^{\prime}}$ and an additional parameter $\tan \bar{\beta}$. A detailed summary of these results is clearly beyond the scope of this review. We refer to the numerous plots in this paper where it can be found how the results on FCNCs in 331 models listed above, in particular correlations between various observables, are modified by these new contributions. As a byproduct we analyzed there for the first time the ratio $\varepsilon^{\prime} / \varepsilon$ in these models including both $Z^{\prime}$ and $Z$ contributions. Our analysis of electroweak precision observables within 331 models demonstrates transparently that the interplay of NP effects in electroweak precision observables and those in flavour observables could allow in the future to identify the favourite 331 model. 


\subsubsection{Littlest Higgs Model with T-parity}

As stressed in Section 3.6 of [20] the LHCb data can be considered as a relief for this model.

- In this model it was not possible to obtain $S_{\psi \phi}$ of $\mathcal{O}(1)$ and values above 0.3 were rather unlikely. In this model also negative values for $S_{\psi \phi}$ as opposed to $2 \mathrm{HDM}_{\overline{\mathrm{MFV}}}$ are possible.

- Because of new sources of flavour violation originating in the presence of mirror quarks and new mixing matrices, the usual CMFV relations between $K, B_{d}$ and $B_{s}$ systems are violated. This allows to remove the $\varepsilon_{K}-S_{\psi K_{S}}$ anomaly for both scenarios of $\left|V_{u b}\right|$ and also improve agreement with $\Delta M_{s, d}$.

- The small value of $S_{\psi \phi}$ from $\mathrm{LHCb}$ allows still for sizable enhancements of $\mathcal{B}\left(K_{L} \rightarrow\right.$ $\left.\pi^{0} \nu \bar{\nu}\right)$ and $\mathcal{B}\left(K^{+} \rightarrow \pi^{+} \nu \bar{\nu}\right)$ which would not be possible otherwise.

- On the other hand rare $B$-decays turn out to be SM-like but still some enhancements are possible. In particular $\mathcal{B}\left(B_{s} \rightarrow \mu^{+} \mu^{-}\right)$can be enhanced by $30 \%$ and a significant part of this enhancement comes from the T-even sector. The effects in $\mathcal{B}\left(B_{d} \rightarrow \mu^{+} \mu^{-}\right)$can be larger and also suppression is possible.

\subsubsection{The SM with Sequential Fourth Generation (SM4)}

The LHC data indicate that our nature seems to have only three sequential generations of quarks and leptons. The authors of [416] performed a statistical analysis including the latest Higgs search results and electroweak precision observables and concluded that the SM4 is already excluded at roughly $5 \sigma$. Here we mention nevertheless few interesting signatures of this model after the LHCb data as far as flavour violation is concerned:

- As before the presence of new sources of flavour violations allows to remove all existing tensions related to $\Delta F=2$ observables.

- The small value of $S_{\psi \phi}$ and the results for $\mathcal{B}\left(B_{s} \rightarrow \mu^{+} \mu^{-}\right)$from LHCb imply now that $\mathcal{B}\left(B_{d} \rightarrow \mu^{+} \mu^{-}\right)$can significantly depart from its SM value. On the other hand $\mathcal{B}\left(B_{s} \rightarrow \mu^{+} \mu^{-}\right)$is SM-like with values below SM prediction being more likely than above it.

- Possible enhancements of $\mathcal{B}\left(K^{+} \rightarrow \pi^{+} \nu \bar{\nu}\right)$ and $\mathcal{B}\left(K_{L} \rightarrow \pi^{0} \nu \bar{\nu}\right)$ over the SM3 values are still possible.

More details and references to the original literature can be found in Section 3.7 of [20]. 
(b)

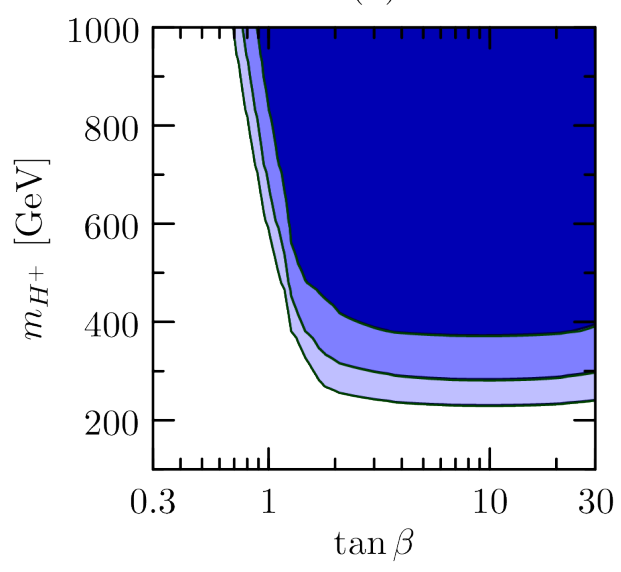

(a)

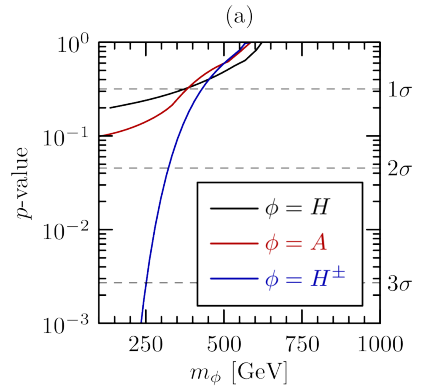

(c)

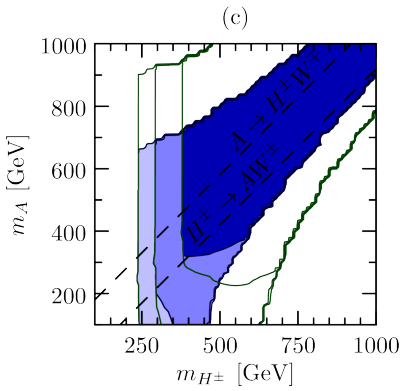

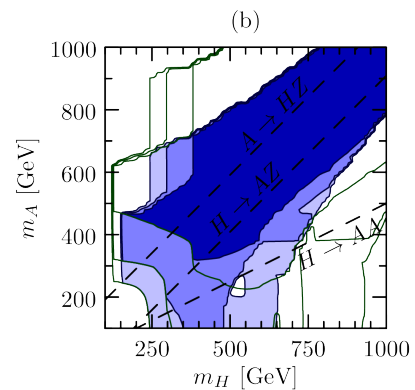

(d)

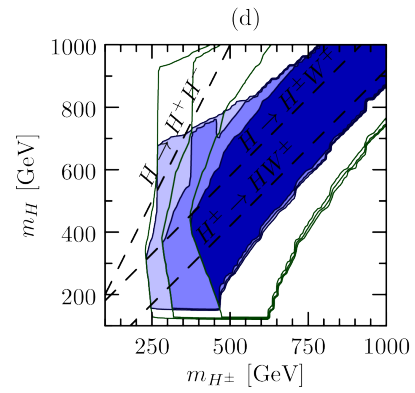

Figure 48: Allowed regions in the $\tan \beta-m_{H^{+}}$plane (left) and mass planes (right). The shaded blue areas are the regions allowed at one, two and three standard deviations (dark to light) 417 .

\subsubsection{CP conserving 2HDM II}

The authors of 417] made a global fit of the CP conserving 2HDM II with a softly broken $Z_{2}$ symmetry. Their analysis includes the experimental constraints from LHC on the mass and signal strength of the Higgs resonance at $126 \mathrm{GeV}$ (which is always interpreted as the light CP-even 2HDM Higgs boson $h$ ), the non-observation of additional Higgs resonances, EWPO and flavour data on $B^{0}-\bar{B}^{0}$ mixing and $B \rightarrow X_{s} \gamma$. Furthermore theoretical constraints are taken into account: vacuum stability and perturbativity. They find that the parameter region with $\beta-\alpha \approx \frac{\pi}{2}$ where the couplings of the light CP-even Higgs boson are SM like is favoured. In Fig. 48 (left) the allowed range in the $\tan \beta-m_{H^{+}}$plane is shown. The lower bound on $m_{H^{+}}$of $322 \mathrm{GeV}(400 \mathrm{GeV})$ at $2 \sigma(1 \sigma)$ for $\tan \beta>1$ follows from the constraint from $B \rightarrow X_{s} \gamma$. On the right hand side of Fig. 48 the allowed mass regions for $H^{0} / A^{0} / H^{+}$is shown. Flavour and EWP observables exclude scenarios with both $m_{H}$ and $m_{A}$ below $300 \mathrm{GeV}$ at $2 \sigma$.

Other recent analyzes of 2HDM II can be found in [418 421]. In [193] it was even stated that $2 \mathrm{HDM}$-II is ruled out by $B \rightarrow D\left(D^{*}\right) \tau \nu$ data. However, it seems to us that such a statement is premature as the data could still change in the future and moreover this would also imply that the SM is ruled out because the 2HDM-II contains the SM in its parameter space in the decoupling limit. 


\subsubsection{Supersymmetric Flavour Models (SF)}

None of the supersymmetric particles has been seen so far. However one of the important predictions of the simplest realization of this scenario, the MSSM with $R$-parity, is a light Higgs with $m_{H} \leq 130 \mathrm{GeV}$. The discovery of a Higgs boson at the LHC around $125 \mathrm{GeV}$ could indeed be the first hints for a Higgs of the MSSM but it will take some time to verify it. In any case MSSM remains still a viable NP scenario at scales $\mathcal{O}(1 \mathrm{TeV})$ although the absence of the discovery of supersymmetric particles is rather disappointing. Similarly the SUSY dreams of large $\mathcal{B}\left(B_{s} \rightarrow \mu^{+} \mu^{-}\right)$and $S_{\psi \phi}$ have not been realized at $\mathrm{LHCb}$ and CMS. However the data from these experiments listed in (59), (133) and (134) have certainly an impact on SUSY predictions.

In view of a rather rich structure of various SF models analyzed in detail in 35 and summarized in Section 3.8 of [20] it is not possible to discuss them adequately here. We make only two comments:

- The new data on $\mathcal{B}\left(B_{s, d} \rightarrow \mu^{+} \mu^{-}\right)$indicate that there is more room for NP contribution dominated by left-handed currents than right-handed currents.

- Although the large range of departures from SM expectations found in [35] has been significantly narrowed, still significant room for novel SUSY effects is present in quark flavour data. Assuming that SUSY particles will be found, the future improved data for $B_{s, d} \rightarrow \mu^{+} \mu^{-}$and $S_{\psi \phi}$ as well as $\gamma$ combined with $\left|V_{u b}\right|$ should help in distinguishing between various supersymmetric flavour models.

\subsubsection{Supersymmetric $\mathrm{SO}(10)$ GUT model}

Grand Unified Theories open the possibility to transfer the neutrino mixing matrix $U_{\text {PMNS }}$ to the quark sector and therefore correlate leptonic and hadronic observables. This is accomplished in a controlled way in a concrete $\mathrm{SO}(10)$ SUSY GUT proposed by Chang, Masiero and Murayama (CMM model) where the atmospheric neutrino mixing angle induces new $b \rightarrow s$ and $\tau \rightarrow \mu$ transitions [422, 423]. In [424] we have performed a global analysis in the CMM model of several flavour processes containing $\Delta M_{s}, S_{\psi \phi}$, $b \rightarrow s \gamma$ and $\tau \rightarrow \mu \gamma$ including an extensive renormalization group (RG) analysis to connect Planck-scale and low-energy parameters. A short summary of this work can also be found in 20,425,426.

Here we want to shortly summarize the basic features of this model. At the Planck scale the flavour symmetry is exact but it is already broken at the $\mathrm{SO}(10)$ scale which manifests itself in the appearance of a non-renormalizable operator in the $\mathrm{SO}(10)$ superpotential. The $\mathrm{SO}(10)$ symmetry is broken down to the $\mathrm{SM}$ gauge group via $\mathrm{SU}(5)$ and the whole $\overline{\mathbf{5}}$-plet $\mathbf{5}_{i}=\left(d_{R i}^{c}, \ell_{L i},-\nu_{\ell_{i}}\right)^{T}$ and the corresponding supersymmetric partners are then rotated by $U_{\mathrm{PMNS}}$. While at $M_{\mathrm{Pl}}$ the soft masses are still universal, we get a large splitting between the masses of the $1^{\text {st }} / 2^{\text {nd }}$ and $3^{\text {rd }}$ down-squark and chargedslepton generation at the electroweak scale due to RG effects of $y_{t}$. The flavour effects 
in the CMM model are then mainly determined by the generated mass splitting and the structure of the PMNS matrix.

In [424] we used tribimaximal mixing in $U_{\text {PMNS }}$. However the latest data now show that the reactor neutrino mixing angle $\theta_{13} \approx 8^{\circ}$ is indeed non-zero. Consequently whereas effects in $K^{0}-\bar{K}^{0}$ mixing, $B_{d}^{0}-\bar{B}_{d}^{0}$ mixing and $\mu \rightarrow e \gamma$ are very small in the original version of the model, this changes when $\theta_{13} \approx 8^{\circ}$ is taken into account. Now large effects in $\mu \rightarrow e \gamma$ are possible. With tribimaximal mixing large contributions were only predicted in observables connecting the $2^{\text {nd }}$ and $3^{\text {rd }}$ generation. So we focused on $b \rightarrow s \gamma, \tau \rightarrow \mu \gamma, \Delta M_{s}$ and $S_{\psi \phi}$. Concerning $B_{s} \rightarrow \mu^{+} \mu^{-}$, effects are small because the CMM model at low energies appears as a special version of the MSSM with small $\tan \beta$ such that this branching ratio stays SM-like. Another observable that needs further investigation is the Higgs mass which in the CMM model tends to be too small. The analysis of [424] was done prior to the detection of the Higgs boson and there we pointed out the Higgs mass could be up to $120 \mathrm{GeV}$ in the parameter range consistent with flavour observables. An updated analysis of the CMM model however shows that the two new experimental results, $\theta_{13} \approx 8^{\circ}$ and $M_{H}=126 \mathrm{GeV}$, put the CMM model under pressure 427, 428]: The constraint from $\mathcal{B}(\mu \rightarrow e \gamma)$ (see Eq. 253p) supersedes those from $b \rightarrow s$ and $\tau \rightarrow \mu \mathrm{FCNC}$ processes and requires very heavy sfermion and gaugino masses $(\approx(8-10) \mathrm{TeV})$. It is very difficult to find a range in the parameter space which simultaneously satisfy the Higgs mass constraint and the experimental upper bound on $\mathcal{B}(\mu \rightarrow e \gamma)$. A Higgs mass of $M_{H}=126 \mathrm{GeV}$ can be accommodated by passing from the MSSM to the NMSSM.

\subsubsection{The Minimal Effective Model with Right-handed Currents: RHMFV}

Few years ago interest in making another look at the right-handed currents in general originated in tensions between inclusive and exclusive determinations of the elements of the CKM matrix $\left|V_{u b}\right|$ and $\left|V_{c b}\right|$. It could be that these tensions are due to the underestimate of theoretical and/or experimental uncertainties. Yet, as pointed out and analyzed in particular in [429, 430], it is a fact that the presence of right-handed currents could either remove or significantly weaken some of these tensions, especially in the case of $\left|V_{u b}\right|$.

In 294 the implications of this idea for other processes have been investigated in an effective theory approach based on a left-right symmetric flavour group $S U(3)_{L} \times$ $S U(3)_{R}$, commuting with an underlying $S U(2)_{L} \times S U(2)_{R} \times U(1)_{B-L}$ global symmetry and broken only by two Yukawa couplings. The model contains a new unitary matrix $\tilde{V}$ controlling flavour-mixing in the RH sector and can be considered as the minimally flavour violating generalization to the $\mathrm{RH}$ sector. Thus bearing in mind that this model contains non-MFV interactions from the point of view of the standard MFV hypothesis that includes only LH charged currents it can be called RHMFV. Referring to 294 for details, we would like to summarize the present status of this model: 
- In this model it is the high inclusive value of $\left|V_{u b}\right|$ that is selected by the model as the true value of this element providing simultaneously the explanation of the smaller $\left|V_{u b}\right|$ found in SM analysis of exclusive decays and very high value of $\left|V_{u b}\right|$ implied by the previous data for $\mathcal{B}\left(B^{+} \rightarrow \tau^{+} \nu_{\tau}\right)$. The decrease of the latter branching ratio casts some doubts on the explanation of the tension between inclusive and exclusive values of $\left|V_{u b}\right|$ by right-handed currents but the large experimental error on $\mathcal{B}\left(B^{+} \rightarrow \tau^{+} \nu_{\tau}\right)$ does not yet exclude this idea. It could be that the true value of $\left|V_{u b}\right|$ determined in inclusive decays is somewhere between its present central inclusive and exclusive values, like $\left|V_{u b}\right|=3.8 \times 10^{-3}$, and that the effect of right-handed currents is smaller than previously anticipated.

- A value like $\left|V_{u b}\right|=3.8 \times 10^{-3}$ still implies $\sin 2 \beta \approx 0.74$ but in this model in the presence of SM-like $S_{\psi \phi}$ measured by LHCb, it is possible due to new phases to achieve the agreement with the experimental value of $S_{\psi K_{S}}$. For $S_{\psi \phi}=\mathcal{O}(1)$ this would not be possible as stressed in 294.

- As far as the decays $B_{s, d} \rightarrow \mu^{+} \mu^{-}$are concerned, already in 2010 the constraint from $B \rightarrow X_{s} \mu^{+} \mu^{-}$precluded $\mathcal{B}\left(B_{s} \rightarrow \mu^{+} \mu^{-}\right)$to be above $1 \cdot 10^{-8}$. Moreover NP effects in $B_{d} \rightarrow \mu^{+} \mu^{-}$have been found generally to be smaller than in $B_{s} \rightarrow \mu^{+} \mu^{-}$. But the smallness of $S_{\psi \phi}$ from LHCb modified the structure of the RH matrix and one should expect that the opposite is true in accordance with the room left for $\mathrm{NP}$ in $B_{d} \rightarrow \mu^{+} \mu^{-}$by the LHCb data. But to be sure a more detailed numerical analysis is required.

There are other interesting consequences of this NP scenario that can be found in [294] and 431] even if some of them will be modified due to changes in the structure of the RH matrix. It looks like RHMFV could still remain a useful framework when more precise experimental data for observables just mentioned will be available in the second half of this decade.

\subsubsection{A Randall-Sundrum Model with Custodial Protection}

Models with a warped extra dimension first proposed by Randall and Sundrum provide a geometrical explanation of the hierarchy between the Planck scale and the EW scale. Moreover, when the SM quarks and leptons are allowed to propagate in the fifth dimension (bulk), these models naturally generate the hierarchies in the fermion masses and mixing angles through different localization of the fermions in the bulk.

In order to avoid problems with electroweak precision tests (EWPT) and FCNC processes, the gauge group is generally larger than the SM gauge group 432,433]:

$$
G_{\mathrm{RSc}}=S U(3)_{c} \times S U(2)_{L} \times S U(2)_{R} \times U(1)_{X}
$$

and similarly to the LHT model new heavy gauge bosons, KK gauge bosons, are present. Moreover, a special choice of fermion representation protects the left-handed flavour 
conserving couplings in order to agree with the data, in particular in the case of $Z \rightarrow b \bar{b}$ [434.

The increased symmetry provides a custodial protection also for left-handed flavour violating couplings of $Z$ to down-quarks and to corresponding right-handed couplings to up-quarks 49, 167,435]. We will call this model RSc to indicate the custodial protection. Detailed analyses of electroweak precision tests and FCNCs in a RS model without and with custodial protection can also be found in [436 438].

The different placing of fermions in the bulk generates non-universal couplings of fermions to KK gauge bosons and $Z$ and after the rotation to mass eigenstates induces FCNC transitions at the tree-level. As we discussed tree-level FCNCs due to exchanges of a single gauge boson $Z^{\prime}$ or $Z$, it is instructive to emphasize the differences between our examples and the RS scenario. These are:

- First of all there are several new heavy gauge bosons. The lightest new gauge bosons are the KK-gluons, the KK-photon and the electroweak KK gauge bosons $W_{H}^{ \pm}, W^{\prime \pm}, Z_{H}$ and $Z^{\prime}$, all with masses $M_{K K}$ at least as large as $2-3 \mathrm{TeV}$ as required by the consistency with the EWPT 432 434].

- While in our simple examples a given gauge boson was the dominant NP effect in $K, B_{s}$ and $B_{d}$ systems, the situation in RSc is different. NP in $\varepsilon_{K}$ is dominated by KK gluons, $B_{s, d}^{0}-\bar{B}_{s, d}^{0}$ systems by KK gluons and KK weak gauge bosons, while rare $K$ and $B_{s, d}$ decays by right-handed flavour-violating couplings of $Z$ to downquarks. Therefore the correlations between $\Delta F=2$ and $\Delta F=1$ observables found in our simple scenarios are absent here.

- Yet, the problematic KK gluon contributions to $\varepsilon_{K}$, requiring some fine-tuning of the parameters have an indirect impact on other observables as the space of parameters is severely reduced. Moreover, the fact that RSc has a goal to explain the masses and mixing angles implies as mentioned below some correlations between different meson systems which were absent in our examples.

A very extensive analysis of FCNCs has been presented prior to the LHCb data in [49, 435]. The branching ratios for $B_{s, d} \rightarrow \mu^{+} \mu^{-}$and $B \rightarrow X_{s, d} \nu \bar{\nu}$ have been found to be SM-like: the maximal enhancements of these branching ratios amount to $15 \%$. This is clearly consistent with the present LHCb and CMS data but the situation may change this year. An anti-correlation in the size of NP effects has been found between $S_{\psi \phi}$ and rare $K$ decays precluding, similar to the LHT model, visible effects in the latter in the presence of a large $S_{\psi \phi}$. The smallness of $S_{\psi \phi}$ are good news for rare $K$ decays in the RSc framework as now sizable enhancements of branching ratios for $K^{+} \rightarrow \pi^{+} \nu \bar{\nu}$ and $K_{L} \rightarrow \pi^{0} \nu \bar{\nu}$ are allowed.

So far so good. In addition to $\varepsilon_{K}$ large NP contributions in the RS framework that require some tunings of parameters in order to be in agreement with the experimental data have been found in $\varepsilon^{\prime} / \varepsilon$ [38, 439. Moreover it appears that the fine tuning in 
this ratio is not necessarily consistent with the one required in the case of $\varepsilon_{K}$. As far as transitions dominated by dipole operators are concerned some fine tuning of NP contributions to EDMs 440, 441] and $\mathcal{B}(\mu \rightarrow e \gamma)$ [442 444] is required. After the recent data from the MEG experiment at PSI [363] this is in particular the case of $\mathcal{B}(\mu \rightarrow e \gamma)$ when considered in conjunction with $\mathcal{B}(\mu \rightarrow 3 e)$ [445]. Sizable contributions are possible also to the $b \rightarrow s \gamma$ transition. However as they affect mostly the chirality-flipped Wilson coefficient $C_{7}^{\prime}, \mathcal{B}\left(B \rightarrow X_{s} \gamma\right)$ remains in good agreement with the data [440, 446, 447].

It appears then that this scenario, unless extended by some flavour symmetry, does not look like a favorite one for NP around few TeV scales. On the other hand many of the ideas and concepts that characterize most of the physics discussed in the context of RS scenario do not rely on the assumption of additional dimensions and as indicated by AdS/CFT correspondence we can regard RS models as a mere computational tool for certain strongly coupled theories. Therefore in spite of some tensions in this NP scenario the techniques developed in the last decade will certainly play an important role in the phenomenology if a new strong dynamics will show up at the LHC after its upgrade.

\subsubsection{Composite Higgs and Partial Compositeness}

This brings us to the idea which still has not been ruled out in spite of the discovery of a boson that looks like the Higgs boson of the SM. The severe fine-tuning problem which this model faces can still be avoided if the Higgs boson is a composite object. Then the question arises how in such a model fermion masses can be generated without at the same time violating the stringent bounds of FCNCs. The most popular mechanism to achieve this goal is an old 4D idea which is known as partial compositeness [448]. In this NP scenario SM fermions couple linearly to heavy composite fermions with the same quantum numbers. The SM fermion masses are then generated in a seesaw-like manner and the mass eigenstates are superpositions of elementary and composite fields. Light quarks are dominantly elementary while the degree of compositeness is large for the top quark.

This idea for explaining the fermion mass hierarchies by hierarchical composite-elementary mixings, already used in RS scenario discussed previously, leads to a suppression of FCNCs even if the strong sector is completely flavour-anarchic [449 451]. Yet, as we have seen in the $5 \mathrm{D}$ setting even this mechanism is not powerful enough to satisfy the bounds from FCNCs without some degree of fine-tuning for the masses of KK gluons, represented here by the resonances of the strong sector, in the reach of the LHC 435, 440, 452. For this reason, various mechanisms have been suggested to further suppress flavour violation. One idea is to impose a flavour symmetry under which the strong sector is invariant and which is only broken by the composite-elementary mixings 88,453 456. Alternative solutions include flavour symmetries broken also in the strong sector [457-459]. Also an extension of the (flavour-blind) global symmetry of the strong sector has been proposed in 460]. 
In addition as we have seen in the case of RSc, protection mechanisms have to be invoked to satisfy electroweak precision tests, in particular related to the $T$ parameter, 432, 433] that requires the extension of the gauge group. In the $4 \mathrm{D}$ setting this means that the strong sector should be invariant under the custodial symmetry $S U(2)_{L} \times S U(2)_{R} \times$ $U(1)_{X}$. Moreover, the presence of heavy vectorial composite fermions that mix with the SM fermions and the presence of new heavy vector resonances implies modifications of $Z$ couplings leading to unacceptable $Z$ coupling to left-handed $b$ quarks and tree-level FCNCs mediated by $Z$. As already discussed in the context of RS a particular choice of fermion representation allows to remove these problems both for $Z \rightarrow b \bar{b}$ [434] and also FCNCs 49, 167, 435. In the 4D setting this is equivalent to making the strong sector (approximately) invariant under a discrete symmetry [434].

The important point to be made here, emphasized also recently by Straub [461], is that the resulting pattern of FCNCs mediated by $Z$ will generally depend on

- The flavour symmetry imposed on the strong sector admitting also the case of an anarchic strong sector,

- Choice of the fermion representations to satisfy the bounds on $Z$ couplings.

A simple 4D effective framework to study the phenomenology of these different possibilities is given by the two-site approach proposed in [462]. In this framework, one considers only one set of fermion resonances with heavy Dirac masses as well as spin1 resonances associated to the global symmetry $S U(3)_{c} \times S U(2)_{L} \times S U(2)_{R} \times U(1)_{X}$ which can be considered as new "heavy gauge bosons". This approach can be viewed as a truncation of 5D warped (RS) models, taking into account only the lightest set of KK states. This approximation has already been used in [49, 167, 435 in the context of RSc as discussed above and is particularly justified in the case when FCNCs appear already at tree-level.

In the language of $4 \mathrm{D}$ strongly coupled theories the RSc scenario discussed previously is custodially protected flavour-anarchic model where the left-handed quarks couple to a single composite fermion. In such a framework NP effects in rare $K$ and $B_{s, d}$ decays as analyzed in [49, 167, 435] are full dominated by $\mathrm{RH} Z$-couplings and the pattern of flavour violation with implied correlations is described by the right DNA chart in Fig. 47 .

However, there are other possibilities [461]. In a custodially protected flavour-anarchic model, where the left-handed up- and down-type quarks couple to two different composite fermions rare $K$ and $B_{s, d}$ decays are full dominated by LH $Z$-couplings. The pattern of flavour violation with implied correlations is summarized by the left DNA chart in Fig. 47. Indeed the results for this scenario in Fig. 4 in [461] can easily be understood on the basis of the DNA-chart in Fig. 47.

Next one can consider a model with partial compositeness in which the strong sector possesses $U(2)^{3}$ flavour symmetry 88,463 , minimally broken by the composite-elementary 
mixings of right-handed quarks. In this case as already discussed at length by us and also seen in Fig. 4 of [461] the pattern of flavour violation with implied correlations is summarized by the DNA-chart in Fig. 46. Useful set of references to models with partial compositeness can be found in 461.

\subsubsection{Gauged Flavour Models}

In these models 234, 464, 465 a MFV-like ansatz is implemented in the context of maximal gauge flavour (MGF) symmetries: in the limit of vanishing Yukawa interactions these gauge symmetries are the largest non-Abelian ones allowed by the Lagrangian of the model. The particle spectrum is enriched by new heavy gauge bosons, carrying neither colour nor electric charges, and exotic fermions to cancel anomalies. Furthermore, the new exotic fermions give rise to the SM fermion masses through a seesaw mechanism, in a way similar to how the light left-handed (LH) neutrinos obtain masses by the heavy RH ones.

Even if this approach has some similarities to the usual MFV description, the presence of flavour-violating neutral gauge bosons and exotic fermions introduces modifications of the SM couplings and tends to lead to dangerous contributions to FCNC processes mediated by the new heavy particles.

In 135 a detailed analysis of $\Delta F=2$ observables and of $B \rightarrow X_{s} \gamma$ in the framework of a specific MGF model of Grinstein et al. [464 including all relevant contributions has been presented. The number of parameters in this model is much smaller than in some of the extensions of the SM discussed above and therefore it is not obvious that the present tensions on the flavour data can be removed or at least softened. Therefore it is of interest to summarize the status of this model in the light of the discussions of FCNCs in the previous sections. The situation is as follows:

- After imposition of the constraint from $\varepsilon_{K}$ only small deviations from the SM values of $S_{\psi K_{s}}$ and $S_{\psi \phi}$ are allowed. While at the time of our analysis in [135] this appeared as a possible problem, this result is fully consistent with present $\mathrm{LHCb}$ data. Consequently this model selects the scenario with exclusive (small) value of $\left|V_{u b}\right|$.

- The structure of correlations between $\Delta F=2$ observables is very similar to models with CMFV and represented by the DNA-chart in Fig. 45. In particular $\left|\varepsilon_{K}\right|$ is enhanced without modifying $S_{\psi K_{S}}$. Moreover, $\Delta M_{d}$ and $\Delta M_{s}$ are strongly correlated in this model with $\varepsilon_{K}$ and the enhancement of the latter implies the enhancement of $\Delta M_{s, d}$. In fact the $\varepsilon_{K}-\Delta M_{s, d}$ tension discussed at length in Step 3 of our review has been pointed out in [135]. Thus the future of this model depends on the values of $\left|V_{c b}\right|$ and a of number of non-perturbative parameters as analyzed in 45 
- However, the main problem of this scenario in 2011, the branching ratio for $B^{+} \rightarrow$ $\tau^{+} \nu_{\tau}$, that in this model is in the ballpark of $0.7 \times 10^{-4}$, softened significantly in view of the 2012 data from Belle.

\subsubsection{New Vectorlike Fermions: a Minimal Theory of Fermion Masses}

We end the review of NP models by summarizing the results obtained within a model with vectorlike fermions based on [42, 466] that can be seen as a Minimal Theory of Fermion Masses (MTFM). The idea is to explain SM fermion masses and mixings by their dynamical mixing with new heavy vectorlike fermion $F$. Very simplified the Lagrangian has the following form: $\mathcal{L} \propto m \bar{f} F+M \bar{F} F+\lambda h F F$, where $M$ denotes the heavy mass scale, $m$ characterizes the mixing and $\lambda$ is a Yukawa coupling. Thus the light fermions have an admixture of heavy fermions with explicit mass terms.

This mass generation mechanism bears some similarities to the one in models with partial compositeness and gauge flavour models just discussed. As in this model the Higgs couples only to vectorlike heavy fermions but not to chiral fermions of the SM, that SM Yukawas arise solely through mixing. We reduce the number of parameters such that it is still possible to reproduce the SM Yukawa couplings and that at the same time flavour violation is suppressed. In this way we can identify the minimal FCNC effects. A central formula is the leading order expression for the SM quark masses

$$
m_{i j}^{X}=v \varepsilon_{i}^{Q} \varepsilon_{j}^{X} \lambda_{i j}^{X}, \quad(X=U, D), \quad \varepsilon_{i}^{Q, U, D}=\frac{m_{i}^{Q, U, D}}{M_{i}^{Q, U, D}} .
$$

In [466] the heavy Yukawa couplings $\lambda^{U, D}$ have been assumed to be anarchical $\mathcal{O}(1)$ real numbers which allowed a first look at the phenomenological implications. In [42] the so called TUM (Trivially Unitary Model) was studied in more detail. We assumed universality of heavy masses $M_{i}^{Q}=M_{i}^{U}=M_{i}^{D}=M$ and unitary Yukawa matrices. With this the flavour structure simplified considerably. Furthermore we concentrated on flavour violation in the down sector and thus set $\lambda^{U}=\mathbb{1}$. After fitting the SM quark masses and the CKM matrix we are left with only four new real parameters and no new phases: $M, \varepsilon_{3}^{Q}, s_{13}^{d}, s_{23}^{d}$. The latter two parameters are angles of $\lambda^{D}$ (the third angle is fixed by the fitting procedure) and from fitting $m_{t}$ it follows that $0.8 \leq \varepsilon_{3}^{Q} \leq 1$.

The new contributions to FCNC processes are dominated by tree-level flavour violating $Z$ couplings to quarks. The simplest version of the MTFM, the TUM, is capable of describing the known quark mass spectrum and the elements of the CKM matrix favouring $\left|V_{u b}\right| \approx 0.0037$. Since there are no new phases in the TUM $S_{\psi K_{S}}$ stays SM-like and thus the large inclusive value of $\left|V_{u b}\right|$ is disfavored. Although effects in $\varepsilon_{K}$ can in principle be large, the effects are bounded by $\mathcal{B}\left(K_{L} \rightarrow \mu^{+} \mu^{-}\right)_{\mathrm{SD}} \leq 2.5 \cdot 10^{-9}$. For a $\left|V_{u b}\right|$ in between exclusive and inclusive value it is still possible to find regions in the parameter space that satisfy Eq. (96) and 212 but then the prediction of the model is that $S_{\psi K_{S}} \approx 0.72$ which is by $2 \sigma$ higher than its present experimental central value. 

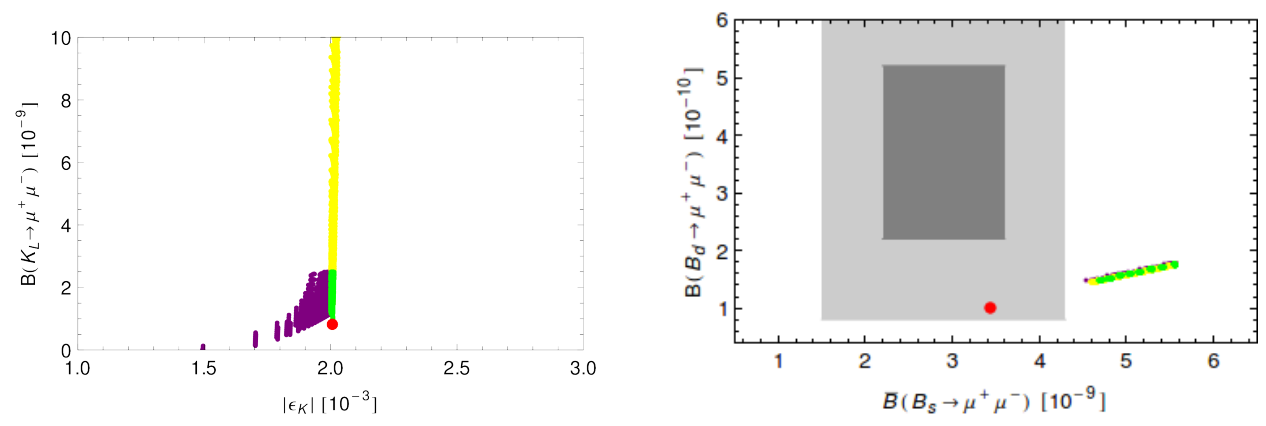

Figure 49: $\mathcal{B}\left(K_{L} \rightarrow \mu^{+} \mu^{-}\right)$vs. $\left|\varepsilon_{K}\right|$ and $\mathcal{B}\left(B_{d} \rightarrow \mu^{+} \mu^{-}\right)$vs. $\overline{\mathcal{B}}\left(B_{s} \rightarrow \mu^{+} \mu^{-}\right)$for $M=3$ TeV and $\left|V_{u b}\right|=0.0037$. Green points are compatible with both bounds for $\left|\varepsilon_{K}\right|$ (96) and $\mathcal{B}\left(K_{L} \rightarrow \mu^{+} \mu^{-}\right)$(212), yellow is only compatible with $\left|\varepsilon_{K}\right|$ and purple only with $\mathcal{B}\left(K_{L} \rightarrow \mu^{+} \mu^{-}\right)$. The red point corresponds to the $S M$ central value. The dark/light gray range shows the overlap of the $1 \sigma / 2 \sigma$ experimental values of $\mathcal{B}\left(B_{d} \rightarrow \mu^{+} \mu^{-}\right)$vs. $\overline{\mathcal{B}}\left(B_{s} \rightarrow \mu^{+} \mu^{-}\right)$.

In Fig. 49 (left) we show the correlation $\mathcal{B}\left(K_{L} \rightarrow \mu^{+} \mu^{-}\right)$vs. $\left|\varepsilon_{K}\right|$ for $M=3 \mathrm{TeV}$ where only the green points satisfy (212) and (96) simultaneously. In the TUM effects in $B_{s, d}$ mixings are negligible and the pattern of deviations from SM predictions in rare $B$ decays is CMFV-like as can be see on the right hand side of Fig. 49. However $\mathcal{B}\left(B_{s, d} \rightarrow \mu^{+} \mu^{-}\right)$are uniquely enhanced over their SM values. For $M=3 \mathrm{TeV}$ these enhancements amount to at least $35 \%$ and can be as large as a factor of two. With increasing $M$ the enhancements decrease. However they remain sufficiently large for $M \leq 5 \mathrm{TeV}$ to be detected in the flavour precision era. Also effects in $K \rightarrow \pi \nu \bar{\nu}$ transitions are enhanced by a similar amount.

At the time when our paper was published there was a hope that the enhancement of $\mathcal{B}\left(B_{s} \rightarrow \mu^{+} \mu^{-}\right)$uniquely predicted by the model would be confirmed by the improved data. As seen on the right hand side of Fig. 49 the most recent data from LHCb and CMS do not support this prediction and either the value of $M$ has to be increased or the TUM has to be made less trivial.

\section{Summary and Shopping List}

Our review of strategies for the identification of New Physics through quark flavour violating processes is approaching the end. In the spirit of our previous reviews 12 , 17,20 we have addressed the question how in principle one could identify NP with the help of quark flavour violating processes. In contrast to [12,17] we have concentrated on the simplest extensions of the SM, describing more complicated ones only in the final part of this review. These simple constructions are helpful in identifying certain patterns of flavour violation. In particular correlations between various observables characteristic for these scenarios can distinguish between them. These features are 
exposed compactly by the DNA-charts in Figs. 45,47. Our extensive study of models in which flavour violation is governed by tree-level exchanges of gauge bosons, scalars and pseudoscalars with different couplings exemplified by LHS, RHS, LRS and ALRS scenarios shows that future measurements can tell us which one of them is favoured by nature.

However we are aware of the fact that these simple scenarios are not fully representative for more complicated models in which a collection of several new particles and a number of new parameters can wash out various correlations identified by us. This is in particular the case of models in which FCNCs appear first at one-loop level and the FCNC amplitudes depend on the masses of exchanged gauge bosons, fermions and scalars and their couplings to SM particles. In CMFV, MFV at large and models with $U(2)^{3}$ some general pattern of flavour violation can still be identified. But this is much harder in the case of models with non-MFV contributions.

Our review shows that without some concrete signs of NP in high energy collisions at the LHC a successful execution of the whole strategy presented in this review will be challenging. On the other hand with many observables accurately measured some picture of the physics beyond the LHC scales could in principle emerge from flavour physics and rare processes alone. Yet, there is still a hope that the second half of this decade will bring the discoveries of new particles at the LHC and this would give us some concrete directions for the next steps through flavour physics that would allow us to get a better indirect insight into the physics at short distance scales outside the reach of the LHC.

We end our review with a short shopping list which involves only quark flavour observables:

- Precise values of all non-perturbative parameters relevant for $\Delta F=2$ transitions from lattice QCD. This means also hadronic matrix elements of new operators outside the framework of CMFV. In fact this will be the progress made in the coming years when most of the experiments will sharpen their tools for the second half of this decade.

- Precise determinations of CKM parameters from tree-level decays. This goal will be predominantly addressed by SuperKEKB but in the case of the angle $\gamma$, LHCb will provide a very important contribution.

- Precise values of $S_{\psi K_{S}}$ and $S_{\psi \phi}$ together with improved understanding of hadronic uncertainties represented by QCD penguins.

- Precise measurements of $\mathcal{B}\left(B_{s} \rightarrow \mu^{+} \mu^{-}\right)$and $\mathcal{B}\left(B_{d} \rightarrow \mu^{+} \mu^{-}\right)$. It is important that both branching are measured as this in the interplay with $\Delta M_{s}$ and $\Delta M_{d}$ and precise values of $\hat{B}_{B_{s}}$ and $\hat{B}_{B_{d}}$ would provide a powerful test of CMFV. It is evident from our presentation that the observables related to the time dependent rate would by far enrich these studies. 
- Precise measurements of the multitude of angular observables in $B \rightarrow K\left(K^{*}\right) \ell^{+} \ell^{-}$ accompanied by improved form factors can still provide important information about NP. In particular it will be important to clarify the anomalies observed recently by the LHCb experiment as discussed in Step 7 of our strategy.

- Precise measurements of $\mathcal{B}\left(K^{+} \rightarrow \pi^{+} \nu \bar{\nu}\right)$ and $\mathcal{B}\left(K_{L} \rightarrow \pi^{0} \nu \bar{\nu}\right)$. The first messages will come from NA62 and then hopefully from J-Parc and ORKA.

- Precise measurements of the branching ratios for the trio $B \rightarrow X_{s} \nu \bar{\nu}, B \rightarrow K^{*} \nu \bar{\nu}$ and $B \rightarrow K \nu \bar{\nu}$. These decays are in the hands of SuperKEKB.

- Precise determination of $\mathcal{B}\left(B^{+} \rightarrow \tau^{+} \nu_{\tau}\right)$, again in the hands of SuperKEKB.

- Precise measurement of $\mathcal{B}\left(B \rightarrow X_{s} \gamma\right)$.

- Precise lattice results for the parameters $B_{6}^{(1 / 2)}$ and $B_{8}^{(3 / 2)}$ entering the evaluation of $\varepsilon^{\prime} / \varepsilon$.

A special role will be played by charm physics as it allows us to learn more about flavour physics in the up-quark sector. But the future of this field will depend on the progress on reduction of the hadronic uncertainties.

Next a very important role in the search for NP, as discussed in Step 12, will be played by lepton flavour violating decays, EDMs and $(g-2)_{e, \mu}$. But this is another story and we discussed these topics only very briefly in our review.

Finally a crucial role in these investigations will be played by theorists, both in the case of inventing new ideas for identifying new physics and constructing new extensions of the Standard Model with fewer parameters and thereby more predictive.

In any case this decade is expected to bring a big step forward in the search for new particles and new forces and we should hope that one day the collaboration of experimentalists and theorists will enable us to get some insight into the Zeptouniverse.

\section{Acknowledgements}

We would like to thank all our collaborators for exciting time we had together while exploring different avenues beyond the Standard Model. In connection with this review we thank in particular Michael Ramsey-Musolf for illuminating discussions about EDMs and Wolfgang Altmannshofer for detailed comments on $b \rightarrow s \ell^{+} \ell^{-}$. We also thank Bob Bernstein, Monika Blanke, Christoph Bobeth, Gerhard Buchalla, Svjetlana Fajfer, Mikolaj Misiak, David Straub and Cecilia Tarantino for useful informations. This research was dominantly financed and done in the context of the ERC Advanced Grant project "FLAVOUR" (267104). It was also partially supported by the DFG cluster of excellence "Origin and Structure of the Universe". 


\section{References}

[1] N. Cabibbo, Unitary Symmetry and Leptonic Decays, Phys. Rev. Lett. 10 (1963) $531-532$.

[2] M. Kobayashi and T. Maskawa, CP Violation in the Renormalizable Theory of Weak Interaction, Prog. Theor. Phys. 49 (1973) 652-657.

[3] B. Pontecorvo, Inverse beta processes and nonconservation of lepton charge, Sov. Phys. JETP 7 (1958) 172-173.

[4] Z. Maki, M. Nakagawa, and S. Sakata, Remarks on the unified model of elementary particles, Prog. Theor. Phys. 28 (1962) 870.

[5] S. L. Glashow, J. Iliopoulos, and L. Maiani, Weak Interactions with Lepton-Hadron Symmetry, Phys. Rev. D2 (1970) 1285-1292.

[6] M. Artuso et. al., B, D and K decays, Eur. Phys. J. C57 (2008) 309-492, arXiv:0801.1833.

[7] M. Raidal et. al., Flavour physics of leptons and dipole moments, Eur. Phys. J. C57 (2008) 13-182, arXiv:0801.1826].

[8] M. Antonelli, D. M. Asner, D. A. Bauer, T. G. Becher, M. Beneke, et. al., Flavor Physics in the Quark Sector, Phys.Rept. 494 (2010) 197-414, arXiv:0907.5386.

[9] SuperB Collaboration Collaboration, M. Bona et. al., SuperB: A High-Luminosity Asymmetric $e^{+} e^{-}$Super Flavor Factory. Conceptual Design Report, arXiv:0709.0451.

[10] T. E. Browder, T. Gershon, D. Pirjol, A. Soni, and J. Zupan, New Physics at a Super Flavor Factory, Rev.Mod.Phys. 81 (2009) 1887-1941, arXiv:0802.3201.

[11] The LHCb Collaboration Collaboration, B. Adeva et. al., Roadmap for selected key measurements of LHCb, arXiv:0912.4179.

[12] A. J. Buras, Flavour Theory: 2009, PoS EPS-HEP2009 (2009) 024, arXiv:0910.1032.

[13] G. Isidori, Y. Nir, and G. Perez, Flavor Physics Constraints for Physics Beyond the Standard Model, Ann.Rev.Nucl.Part.Sci. 60 (2010) 355, arXiv:1002.0900.

[14] R. Fleischer, B Physics in the LHC Era: Selected Topics, Nucl.Phys.Proc.Suppl. 209 (2010) 3-8, arXiv:1010.0496.

[15] Y. Nir, Flavour physics and CP violation, arXiv:1010.2666. 
[16] T. Hurth and M. Nakao, Radiative and Electroweak Penguin Decays of B Mesons, Ann.Rev.Nucl.Part.Sci. 60 (2010) 645-677, arXiv:1005.1224.

[17] A. J. Buras, Minimal flavour violation and beyond: Towards a flavour code for short distance dynamics, Acta Phys.Polon. B41 (2010) 2487-2561, arXiv:1012.1447].

[18] M. Ciuchini and A. Stocchi, Physics Opportunities at the Next Generation of Precision Flavor Physics, Ann.Rev.Nucl.Part.Sci. 61 (2011) 491-517, arXiv:1110.3920.

[19] B. Meadows, M. Blanke, A. Stocchi, A. Drutskoy, A. Cervelli, et. al., The impact of SuperB on flavour physics, arXiv:1109.5028.

[20] A. J. Buras and J. Girrbach, BSM models facing the recent LHCb data: A First look, Acta Phys.Polon. B43 (2012) 1427, $\operatorname{arXiv:1204.5064\text {.}}$

[21] G. Borissov, R. Fleischer, and M.-H. Schune, Rare Decays and CP Violation in the $B_{s}$ System, Ann.Rev.Nucl.Part.Sci. 63 (2013) 205-235, arXiv:1303.5575|.

[22] LHCb Collaboration Collaboration, R. Aaij et. al., Implications of LHCb measurements and future prospects, EPJ C 73 (2013) 2373, arXiv:1208.3355.

[23] J. Hewett, H. Weerts, R. Brock, J. Butler, B. Casey, et. al., Fundamental Physics at the Intensity Frontier, arXiv:1205.2671.

[24] T. Hurth and F. Mahmoudi, New physics search with flavour in the LHC era, Rev.Mod.Phys. 85 (2013) 795, arXiv:1211.6453.

[25] S. Stone, New physics from avour, PoS ICHEP2012 (2013) 033, arXiv:1212.6374.

[26] G. Isidori, Flavor physics and CP violation, arXiv:1302.0661.

[27] A. S. Kronfeld, R. S. Tschirhart, U. Al-Binni, W. Altmannshofer, C. Ankenbrandt, et. al., Project X: Physics Opportunities, arXiv:1306.5009.

[28] V. Cirigliano and M. J. Ramsey-Musolf, Low Energy Probes of Physics Beyond the Standard Model, Prog.Part.Nucl.Phys. 71 (2013) 2-20, arXiv:1304.0017.

[29] J. Charles, S. Descotes-Genon, Z. Ligeti, S. Monteil, M. Papucci, et. al., Future sensitivity to new physics in $B_{d}, B_{s}$ and $K$ mixings, arXiv:1309.2293.

[30] Quark Flavor Physics Working Group Collaboration, J. Butler et. al., Report of the Quark Flavor Physics Working Group, arXiv:1311.1076.

[31] G. C. Branco, L. Lavoura, and J. P. Silva, CP Violation, Int.Ser.Monogr.Phys. 103 (1999) 1-536. 
[32] I. I. Bigi and A. Sanda, CP violation, Camb.Monogr.Part.Phys.Nucl.Phys.Cosmol. 9 (2000) 1-382.

[33] E. Lunghi and A. Soni, Possible Indications of New Physics in $B_{d}$-mixing and in $\sin (2 \beta)$ Determinations, Phys. Lett. B666 (2008) 162-165, arXiv:0803.4340.

[34] A. J. Buras and D. Guadagnoli, Correlations among new CP violating effects in $\Delta F=2$ observables, Phys. Rev. D78 (2008) 033005, arXiv:0805.3887].

[35] W. Altmannshofer, A. J. Buras, S. Gori, P. Paradisi, and D. M. Straub, Anatomy and Phenomenology of FCNC and CPV Effects in SUSY Theories, Nucl.Phys. B830 (2010) 17-94, arXiv:0909.1333.

[36] M. Blanke, Insights from the Interplay of $K \rightarrow \pi \nu \bar{\nu}$ and $\epsilon_{K}$ on the New Physics Flavour Structure, Acta Phys.Polon. B41 (2010) 127, arXiv:0904.2528.

[37] W. Altmannshofer, P. Paradisi, and D. M. Straub, Model-Independent Constraints on New Physics in b $\rightarrow$ s $\gamma$ Transitions, JHEP 1204 (2012) 008, arXiv:1111.1257.

[38] W. Altmannshofer and D. M. Straub, Cornering New Physics in $b \rightarrow s$ Transitions, JHEP 1208 (2012) 121, arXiv: 1206.0273.

[39] A. J. Buras and J. Girrbach, On the Correlations between Flavour Observables in Minimal U(2) ${ }^{3}$ Models, JHEP 1301 (2013) 007, |arXiv:1206.3878].

[40] A. J. Buras, F. De Fazio, and J. Girrbach, The Anatomy of $Z$ ' and $Z$ with Flavour Changing Neutral Currents in the Flavour Precision Era, JHEP 1302 (2013) 116, arXiv:1211.1896.

[41] A. J. Buras, F. De Fazio, J. Girrbach, and M. V. Carlucci, The Anatomy of Quark Flavour Observables in 331 Models in the Flavour Precision Era, JHEP 1302 (2013) 023, arXiv:1211.1237.

[42] A. J. Buras, J. Girrbach, and R. Ziegler, Particle-Antiparticle Mixing, CP Violation and Rare $K$ and $B$ Decays in a Minimal Theory of Fermion Masses, JHEP 1304 (2013) 168, arXiv:1301.5498].

[43] A. J. Buras, R. Fleischer, J. Girrbach, and R. Knegjens, Probing New Physics with the $B_{s} \rightarrow \mu^{+} \mu^{-}$Time-Dependent Rate, JHEP 1307 (2013) 77, arXiv:1303.3820.

[44] A. J. Buras, F. De Fazio, J. Girrbach, R. Knegjens, and M. Nagai, The Anatomy of Neutral Scalars with FCNCs in the Flavour Precision Era, JHEP 1306 (2013) 111, arXiv:1303.3723. 
[45] A. J. Buras and J. Girrbach, Stringent Tests of Constrained Minimal Flavour Violation through $\Delta F=2$ Transitions, The European Physical Journal C 9 (73) 2013, arXiv: 1304.6835.

[46] A. J. Buras and J. Girrbach, Left-handed Z' and Z FCNC quark couplings facing new $b \rightarrow s \mu^{+} \mu^{-}$data, JHEP 1312 (2013) 009, arXiv:1309.2466.

[47] A. J. Buras, F. De Fazio, and J. Girrbach, 331 models facing new $b \rightarrow s \mu^{+} \mu^{-}$ data, JHEP 1402 (2014) 112, arXiv:1311.6729.

[48] M. Blanke et. al., Rare and CP-violating $K$ and $B$ decays in the Littlest Higgs model with T-parity, JHEP 01 (2007) 066, hep-ph/0610298.

[49] M. Blanke, A. J. Buras, B. Duling, K. Gemmler, and S. Gori, Rare K and B Decays in a Warped Extra Dimension with Custodial Protection, JHEP 03 (2009) 108, arXiv:0812.3803].

[50] G. Buchalla, A. J. Buras, and M. E. Lautenbacher, Weak decays beyond leading logarithms, Rev.Mod.Phys. 68 (1996) 1125-1144, hep-ph/9512380].

[51] A. J. Buras, Weak Hamiltonian, CP violation and rare decays, hep-ph/9806471. In 'Probing the Standard Model of Particle Interactions', F.David and R. Gupta, eds., 1998, Elsevier Science B.V.

[52] A. J. Buras, Climbing NLO and NNLO Summits of Weak Decays, arXiv:1102.5650.

[53] A. J. Buras, P. Gambino, M. Gorbahn, S. Jager, and L. Silvestrini, Universal unitarity triangle and physics beyond the standard model, Phys. Lett. B500 (2001) 161-167, hep-ph/0007085.

[54] A. J. Buras, Minimal flavor violation, Acta Phys. Polon. B34 (2003) 5615-5668, hep-ph/0310208|.

[55] M. Blanke, A. J. Buras, D. Guadagnoli, and C. Tarantino, Minimal Flavour Violation Waiting for Precise Measurements of $\Delta M_{s}, S_{\psi \phi}, A_{S L}^{s},\left|V_{u b}\right|, \gamma$ and $B_{s, d}^{0} \rightarrow \mu^{+} \mu^{-}$, JHEP 10 (2006) 003, hep-ph/0604057.

[56] T. Inami and C. Lim, Effects of Superheavy Quarks and Leptons in Low-Energy Weak Processes $K_{L} \rightarrow \mu^{+} \mu^{-}, K^{+} \rightarrow \pi^{+} \nu \bar{\nu}$ and $K^{0}-\bar{K}^{0}$, Prog.Theor.Phys. 65 (1981) 297.

[57] G. Buchalla, A. J. Buras, and M. K. Harlander, Penguin box expansion: Flavor changing neutral current processes and a heavy top quark, Nucl. Phys. B349 (1991) $1-47$. 
[58] A. J. Buras, M. E. Lautenbacher, and G. Ostermaier, Waiting for the top quark mass, $K^{+} \rightarrow \pi^{+} \nu \bar{\nu}, B_{s}^{0}-\bar{B}_{s}^{0}$ mixing and $C P$ asymmetries in $B$ decays, Phys. Rev. D50 (1994) 3433-3446, hep-ph/9403384.

[59] A. J. Buras and R. Buras, A Lower bound on sin 2 beta from minimal flavor violation, Phys.Lett. B501 (2001) 223-230, hep-ph/0008273.

[60] M. Blanke and A. J. Buras, Lower bounds on $\Delta M_{s, d}$ from constrained minimal flavour violation, JHEP 0705 (2007) 061, hep-ph/0610037.

[61] A. J. Buras, Relations between $\Delta M_{s, d}$ and $B_{s, d} \rightarrow \mu^{+} \mu^{-}$in models with minimal flavour violation, Phys. Lett. B566 (2003) 115-119, [hep-ph/0303060].

[62] N. Carrasco, M. Ciuchini, P. Dimopoulos, R. Frezzotti, V. Gimenez, et. al., B-physics from $N f=2$ tmQCD: the Standard Model and beyond, arXiv:1308.1851.

[63] G. D’Ambrosio, G. F. Giudice, G. Isidori, and A. Strumia, Minimal flavour violation: An effective field theory approach, Nucl. Phys. B645 (2002) 155-187, hep-ph/0207036.

[64] T. Feldmann and T. Mannel, Minimal Flavour Violation and Beyond, JHEP 0702 (2007) 067, hep-ph/0611095.

[65] G. Colangelo, E. Nikolidakis, and C. Smith, Supersymmetric models with minimal flavour violation and their running, Eur.Phys.J. C59 (2009) 75-98, arXiv:0807.0801.

[66] P. Paradisi, M. Ratz, R. Schieren, and C. Simonetto, Running minimal flavor violation, Phys.Lett. B668 (2008) 202-209, |arXiv:0805.3989|.

[67] L. Mercolli and C. Smith, EDM constraints on flavored CP-violating phases, Nucl.Phys. B817 (2009) 1-24, arXiv:0902.1949.

[68] T. Feldmann, M. Jung, and T. Mannel, Sequential Flavour Symmetry Breaking, Phys.Rev. D80 (2009) 033003, arXiv:0906.1523.

[69] A. L. Kagan, G. Perez, T. Volansky, and J. Zupan, General Minimal Flavor Violation, Phys.Rev. D80 (2009) 076002, arXiv:0903.1794.

[70] P. Paradisi and D. M. Straub, The SUSY CP Problem and the MFV Principle, Phys.Lett. B684 (2010) 147-153, arXiv:0906.4551].

[71] G. Isidori, B Physics in the LHC Era, arXiv: 1001.3431.

[72] T. Hurth, G. Isidori, J. F. Kamenik, and F. Mescia, Constraints on New Physics in MFV models: A Model-independent analysis of $\Delta F=1$ processes, Nucl. Phys. B808 (2009) 326-346, arXiv:0807.5039. 
[73] G. Isidori and D. M. Straub, Minimal Flavour Violation and Beyond, Eur.Phys.J. C72 (2012) 2103, arXiv:1202.0464.

[74] S. Baek and P. Ko, Probing SUSY induced CP violations at B factories, Phys.Rev.Lett. 83 (1999) 488-491, hep-ph/9812229.

[75] S. Baek and P. Ko, Effects of supersymmetric CP violating phases on $B \rightarrow X(s)$ lepton $^{+}$lepton $^{-}$and $\epsilon(K)$, Phys.Lett. B462 (1999) 95-102, hep-ph/9904283.

[76] A. Bartl, T. Gajdosik, E. Lunghi, A. Masiero, W. Porod, et. al., General flavor blind MSSM and CP violation, Phys.Rev. D64 (2001) 076009, hep-ph/0103324.

[77] J. Ellis, J. S. Lee, and A. Pilaftsis, B-Meson Observables in the Maximally CP-Violating MSSM with Minimal Flavour Violation, Phys. Rev. D76 (2007) 115011, arXiv:0708.2079|.

[78] W. Altmannshofer, A. Buras, and P. Paradisi, Low Energy Probes of CP Violation in a Flavor Blind MSSM, Phys.Lett. B669 (2008) 239-245, arXiv:0808.0707.

[79] A. Pich and P. Tuzon, Yukawa Alignment in the Two-Higgs-Doublet Model, Phys.Rev. D80 (2009) 091702, arXiv:0908.1554].

[80] K. Blum, Y. Hochberg, and Y. Nir, Implications of large dimuon CP asymmetry in $B_{d, s}$ decays on minimal flavor violation with low tan $\beta$, JHEP 1009 (2010) 035, arXiv: 1007.1872.

[81] B. A. Dobrescu, P. J. Fox, and A. Martin, CP violation in $B_{s}$ mixing from heavy Higgs exchange, Phys.Rev.Lett. 105 (2010) 041801, arXiv:1005.4238.

[82] W. Altmannshofer and M. Carena, B Meson Mixing in Effective Theories of Supersymmetric Higgs Bosons, Phys.Rev. D85 (2012) 075006, arXiv:1110.0843.

[83] W. Altmannshofer, M. Carena, S. Gori, and A. de la Puente, Signals of CP Violation Beyond the MSSM in Higgs and Flavor Physics, Phys.Rev. D84 (2011) 095027, arXiv:1107.3814.

[84] A. J. Buras, M. V. Carlucci, S. Gori, and G. Isidori, Higgs-mediated FCNCs: Natural Flavour Conservation vs. Minimal Flavour Violation, JHEP 1010 (2010) 009, arXiv: 1005.5310.

[85] G. Branco, P. Ferreira, L. Lavoura, M. Rebelo, M. Sher, et. al., Theory and phenomenology of two-Higgs-doublet models, Phys.Rept. 516 (2012) 1-102, arXiv:1106.0034. 
[86] R. Barbieri, G. Isidori, J. Jones-Perez, P. Lodone, and D. M. Straub, U(2) and Minimal Flavour Violation in Supersymmetry, Eur.Phys.J. C71 (2011) 1725, arXiv:1105.2296.

[87] R. Barbieri, P. Campli, G. Isidori, F. Sala, and D. M. Straub, B-decay CP-asymmetries in SUSY with a $U(2)^{3}$ flavour symmetry, Eur.Phys.J. C71 (2011) 1812, arXiv:1108.5125.

[88] R. Barbieri, D. Buttazzo, F. Sala, and D. M. Straub, Flavour physics from an approximate $U(2)^{3}$ symmetry, JHEP 1207 (2012) 181, arXiv:1203.4218].

[89] R. Barbieri, D. Buttazzo, F. Sala, and D. M. Straub, Less Minimal Flavour Violation, JHEP 1210 (2012) 040, arXiv:1206.1327.

[90] A. Crivellin, L. Hofer, and U. Nierste, The MSSM with a Softly Broken U(2) ${ }^{3}$ Flavor Symmetry, PoS EPS-HEP2011 (2011) 145, arXiv:1111.0246.

[91] A. Crivellin, L. Hofer, U. Nierste, and D. Scherer, Phenomenological consequences of radiative flavor violation in the MSSM, Phys.Rev. D84 (2011) 035030, arXiv:1105.2818.

[92] A. Crivellin and U. Nierste, Supersymmetric renormalisation of the CKM matrix and new constraints on the squark mass matrices, Phys.Rev. D79 (2009) 035018, arXiv:0810.1613.

[93] A. J. Buras and J. Girrbach, Complete NLO QCD Corrections for Tree Level Delta $F=2$ FCNC Processes, JHEP 1203 (2012) 052, arXiv:1201.1302.

[94] A. J. Buras and J. Girrbach, Completing NLO QCD Corrections for Tree Level Non-Leptonic $\Delta F=1$ Decays Beyond the Standard Model, arXiv:1201.2563.

[95] Z. Ligeti, M. Papucci, and G. Perez, Implications of the measurement of the $B_{s}^{0}-\bar{B}_{s}^{0}$ mass difference, Phys. Rev. Lett 97 (2006) 101801, hep-ph/0604112.

[96] G. Buchalla and A. J. Buras, $\sin 2 \beta$ from $k \rightarrow \pi \nu \bar{\nu}$, Phys. Lett. B333 (1994) 221-227, hep-ph/9405259.

[97] A. J. Buras and R. Fleischer, Bounds on the unitarity triangle, $\sin 2 \beta$ and $K \rightarrow \pi \nu \bar{\nu}$ decays in models with minimal flavor violation, Phys. Rev. D64 (2001) 115010, hep-ph/0104238.

[98] L. Wolfenstein, Parametrization of the Kobayashi-Maskawa Matrix, Phys.Rev.Lett. 51 (1983) 1945.

[99] CKMfitter Group Collaboration, J. Charles et. al., CP violation and the CKM matrix: Assessing the impact of the asymmetric $B$ factories, Eur.Phys.J. C41 (2005) 1-131, hep-ph/0406184]. http://www.ckmfitter.in2p3.fr. 
[100] UTfit Collaboration, M. Bona et. al., The UTfit collaboration report on the status of the unitarity triangle beyond the standard model. I: Model-independent analysis and minimal flavour violation, JHEP 03 (2006) 080, hep-ph/0509219. http://www.utfit.org/UTfit/ResultsSummer2013PostEPS.

[101] G. Eigen, G. Dubois-Felsmann, D. G. Hitlin, and F. C. Porter, Global CKM Fits with the Scan Method, PoS ICHEP2012 (2013) 320, [arXiv:1301.5867].

[102] Particle Data Group Collaboration, K. Nakamura et. al., Review of particle physics, J.Phys.G G37 (2010) 075021.

[103] Particle Data Group Collaboration, J. Beringer et. al., Review of Particle Physics (RPP), Phys.Rev. D86 (2012) 010001.

[104] S. Aoki, Y. Aoki, C. Bernard, T. Blum, G. Colangelo, et. al., Review of lattice results concerning low energy particle physics, arXiv:1310.8555.

[105] HPQCD Collaboration Collaboration, R. Dowdall, C. Davies, R. Horgan, C. Monahan, and J. Shigemitsu, B-meson decay constants from improved lattice NRQCD and physical $u, d, s$ and c sea quarks, Phys.Rev.Lett. 110 (2013) 222003, arXiv:1302.2644.

[106] K. Chetyrkin, J. Kuhn, A. Maier, P. Maierhofer, P. Marquard, et. al., Charm and Bottom Quark Masses: An Update, Phys.Rev. D80 (2009) 074010, arXiv:0907.2110.

[107] J. Laiho, E. Lunghi, and R. S. Van de Water, Lattice QCD inputs to the CKM unitarity triangle analysis, Phys. Rev. D81 (2010) 034503, arXiv:0910.2928. Updates available on http://latticeaverages.org/.

[108] HPQCD Collaboration Collaboration, I. Allison et. al., High-Precision Charm-Quark Mass from Current-Current Correlators in Lattice and Continuum QCD, Phys.Rev. D78 (2008) 054513, arXiv:0805.2999].

[109] A. J. Buras, M. Jamin, and P. H. Weisz, Leading and next-to-leading QCD corrections to $\varepsilon$ parameter and $B^{0}-\bar{B}^{0}$ mixing in the presence of a heavy top quark, Nucl. Phys. B347 (1990) 491-536.

[110] J. Urban, F. Krauss, U. Jentschura, and G. Soff, Next-to-leading order QCD corrections for the $B^{0}-\bar{B}^{0}$ mixing with an extended Higgs sector, Nucl. Phys. B523 (1998) 40-58, hep-ph/9710245.

[111] CDF Collaboration, D0 Collaboration Collaboration, T. Aaltonen et. al., Combination of the top-quark mass measurements from the Tevatron collider, Phys.Rev. D86 (2012) 092003, arXiv:1207.1069. 
[112] Heavy Flavor Averaging Group Collaboration, Y. Amhis et. al., Averages of B-Hadron, C-Hadron, and tau-lepton properties as of early 2012, arXiv:1207.1158, http://www.slac.stanford.edu/xorg/hfag.

[113] A. J. Buras, D. Guadagnoli, and G. Isidori, On $\epsilon_{K}$ beyond lowest order in the Operator Product Expansion, Phys.Lett. B688 (2010) 309-313, arXiv:1002.3612.

[114] J. Brod and M. Gorbahn, Next-to-Next-to-Leading-Order Charm-Quark Contribution to the CP Violation Parameter $\varepsilon_{K}$ and $\Delta M_{K}$, Phys.Rev.Lett. 108 (2012) 121801, arXiv:1108.2036.

[115] J. Brod and M. Gorbahn, $\epsilon_{K}$ at Next-to-Next-to-Leading Order: The Charm-Top-Quark Contribution, Phys.Rev. D82 (2010) 094026, arXiv:1007.0684.

[116] P. Gambino and C. Schwanda, Inclusive semileptonic fits, heavy quark masses, and $V_{c b}, \operatorname{arXiv:1307.4551.}$

[117] G. Ricciardi, Determination of the CKM matrix elements - V $(x b)-$, Mod.Phys.Lett. A28 (2013) 1330016, arXiv:1305.2844.

[118] J. A. Bailey, A. Bazavov, C. Bernard, C. Bouchard, C. DeTar, et. al., Update of $\left|V_{c b}\right|$ from the $\bar{B} \rightarrow D^{*} \ell \bar{\nu}$ form factor at zero recoil with three-flavor lattice $Q C D$, arXiv:1403.0635.

[119] A. J. Buras, F. De Fazio, and J. Girrbach, $\Delta I=1 / 2$ Rule, $\varepsilon^{\prime} / \varepsilon$ and $K \rightarrow \pi \nu \bar{\nu}$ in $Z^{\prime}(Z)$ and $G^{\prime}$ Models with FCNC Quark Couplings, arXiv:1404.3824.

[120] A. J. Buras, F. Parodi, and A. Stocchi, The CKM matrix and the unitarity triangle: Another look, JHEP 0301 (2003) 029, hep-ph/0207101.

[121] C. Tarantino, Flavor Lattice QCD in the Precision Era, PoS ICHEP2012 (2013) 023, arXiv:1210.0474.

[122] C. Davies, Standard Model Heavy Flavor physics on the Lattice, PoS LATTICE2011 (2011) 019, arXiv:1203.3862.

[123] E. Gámiz, Flavour physics from lattice QCD, PoS ConfinementX (2012) 241, arXiv:1303.3971.

[124] RBC-UKQCD Collaboration, C. T. Sachrajda, Prospects for Lattice Calculations of Rare Kaon Decay Amplitudes, PoS KAON13 (2013) 019.

[125] N. Christ, Nonleptonic Kaon Decays from Lattice QCD, PoS KAON13 (2013) 029. 
[126] A. J. Buras, S. Jager, and J. Urban, Master formulae for $\Delta F=2 N L O Q C D$ factors in the standard model and beyond, Nucl.Phys. B605 (2001) 600-624, hep-ph/0102316.

[127] RBC and UKQCD Collaborations Collaboration, P. Boyle, N. Garron, and R. Hudspith, Neutral kaon mixing beyond the standard model with $n_{f}=2+1$ chiral fermions, Phys.Rev. D86 (2012) 054028, arXiv:1206.5737.

[128] Collaboration ETM Collaboration, V. Bertone et. al., Kaon Mixing Beyond the $S M$ from $N f=2$ tmQCD and model independent constraints from the UTA, JHEP 1303 (2013) 089, arXiv:1207.1287].

[129] C. Bouchard, E. Freeland, C. Bernard, A. El-Khadra, E. Gamiz, et. al., Neutral $B$ mixing from $2+1$ flavor lattice-QCD: the Standard Model and beyond, PoS LATTICE2011 (2011) 274, arXiv:1112.5642.

[130] SWME Collaboration Collaboration, T. Bae et. al., Neutral kaon mixing from new physics: matrix elements in $N_{f}=2+1$ QCD, arXiv:1309.2040.

[131] M. Blanke, A. J. Buras, K. Gemmler, and T. Heidsieck, $\Delta F=2$ observables and $B \rightarrow X_{q} \gamma$ in the Left-Right Asymmetric Model: Higgs particles striking back, JHEP 1203 (2012) 024, arXiv:1111.5014].

[132] Improved constraints on $\gamma$ from $b^{ \pm} \rightarrow d k^{ \pm}$decays including first results on 2012 data, . Linked to LHCb-ANA-2013-012.

[133] R. Fleischer and R. Knegjens, In Pursuit of New Physics with $B_{s}^{0} \rightarrow K^{+} K^{-}$, Eur.Phys.J. C71 (2011) 1532, arXiv:1011.1096.

[134] LHCb collaboration Collaboration, R. Aaij et. al., Measurement of the CKM angle gamma from a combination of $B \rightarrow$ Dh analyses, arXiv:1305.2050.

[135] A. J. Buras, M. V. Carlucci, L. Merlo, and E. Stamou, Phenomenology of a Gauged SU $(3)^{3}$ Flavour Model, JHEP 1203 (2012) 088, arXiv:1112.4477.

[136] A. J. Buras and J.-M. Gérard, 1/N Expansion for Kaons, Nucl.Phys. B264 (1986) 371.

[137] W. A. Bardeen, A. J. Buras, and J.-M. Gérard, The B Parameter Beyond the Leading Order of 1/N Expansion, Phys.Lett. B211 (1988) 343.

[138] J.-M. Gérard, An upper bound on the Kaon B-parameter and $\operatorname{Re}\left(\epsilon_{K}\right)$, JHEP 1102 (2011) 075, arXiv:1012.2026.

[139] A. J. Buras, J.-M. Gerard, and W. A. Bardeen, Large N Approach to Kaon Decays and Mixing 28 Years Later: $\Delta I=1 / 2$ Rule, $\hat{B}_{K}$ and $\Delta M_{K}$, arXiv:1401.1385. 
[140] A. J. Buras, G. Isidori, and P. Paradisi, EDMs versus CPV in $B_{s, d}$ mixing in two Higgs doublet models with MFV, Phys.Lett. B694 (2011) 402-409, arXiv: 1007.5291.

[141] A. J. Buras, J. Girrbach, and M. Nagai, " $2 \mathrm{HDM}_{\overline{\mathrm{MFV}}}$ Facing Recent LHCb Data." , unpublished.

[142] D. Guadagnoli and G. Isidori, $B R\left(B_{s} \rightarrow \mu^{+} \mu^{-}\right)$as an electroweak precision test, Phys.Lett. B724 (2013) 63-67, |arXiv:1302.3909|.

[143] W. Altmannshofer, The $B_{s}+$ and $B_{d}+$ Decays: Standard Model and Beyond, PoS Beauty2013 (2013) 024, arXiv:1306.0022.

[144] G. Isidori and A. Retico, $B_{s, d} \rightarrow \ell^{+} \ell^{-}$and $K_{L} \rightarrow \ell^{+} \ell^{-}$in SUSY models with non-minimal sources of flavour mixing, JHEP 09 (2002) 063, hep-ph/0208159.

[145] C. Bobeth, A. J. Buras, F. Kruger, and J. Urban, Qcd corrections to $\bar{b} \rightarrow x_{d, s} \nu \bar{\nu}$, $\bar{b}_{d, s} \rightarrow \ell^{+} \ell^{-}, k \rightarrow \pi \nu \bar{\nu}$ and $k_{l} \rightarrow \mu^{+} \mu^{-}$in the mssm, Nucl. Phys. B630 (2002) 87-131, [hep-ph/0112305].

[146] A. Dedes, J. Rosiek, and P. Tanedo, Complete One-Loop MSSM Predictions for $B \rightarrow$ lepton lepton' at the Tevatron and LHC, Phys.Rev. D79 (2009) 055006, arXiv:0812.4320.

[147] S. Descotes-Genon, J. Matias, and J. Virto, An analysis of $B_{d, s}$ mixing angles in presence of New Physics and an update of $B_{s} \rightarrow K^{0 *} \bar{K}^{0 *}$, Phys.Rev. D85 (2012) 034010, arXiv:1111.4882.

[148] K. De Bruyn, R. Fleischer, R. Knegjens, P. Koppenburg, M. Merk, et. al., Branching Ratio Measurements of $B_{s}$ Decays, Phys.Rev. D86 (2012) 014027, arXiv:1204.1735.

[149] K. De Bruyn, R. Fleischer, R. Knegjens, P. Koppenburg, M. Merk, et. al., Probing New Physics via the $B_{s}^{0} \rightarrow \mu^{+} \mu^{-}$Effective Lifetime, Phys.Rev.Lett. 109 (2012) 041801, arXiv:1204.1737.

[150] R. Fleischer, On Branching Ratios of $B_{s}$ Decays and the Search for New Physics in $B_{s}^{0} \rightarrow \mu^{+} \mu^{-}$, Nucl.Phys.Proc.Suppl. 241-242 (2013) 135-140, arXiv:1208.2843.

[151] G. Buchalla and A. J. Buras, The rare decays $K \rightarrow \pi \nu \bar{\nu}, B \rightarrow X \nu \bar{\nu}$ and $B \rightarrow \ell^{+} \ell^{-}:$An Update, Nucl.Phys. B548 (1999) 309-327, [hep-ph/9901288].

[152] M. Misiak and J. Urban, QCD corrections to FCNC decays mediated by Z penguins and $W$ boxes, Phys.Lett. B451 (1999) 161-169, [hep-ph/9901278]. 
[153] G. Buchalla and A. J. Buras, Two-loop large- $m_{t}$ electroweak corrections to $k \rightarrow \pi \nu \bar{\nu}$ for arbitrary higgs boson mass, Phys. Rev. D57 (1998) 216-223, hep-ph/9707243].

[154] C. Bobeth, P. Gambino, M. Gorbahn, and U. Haisch, Complete NNLO QCD analysis of $\bar{B} \rightarrow X_{s} \ell^{+} \ell^{-}$and higher order electroweak effects, JHEP 04 (2004) 071, hep-ph/0312090.

[155] T. Huber, E. Lunghi, M. Misiak, and D. Wyler, Electromagnetic logarithms in $\bar{B} \rightarrow X(s) l^{+} l^{-}$, Nucl.Phys. B740 (2006) 105-137, hep-ph/0512066].

[156] M. Misiak, Rare B-Meson Decays, arXiv:1112.5978.

[157] A. J. Buras, J. Girrbach, D. Guadagnoli, and G. Isidori, On the Standard Model prediction for $B R\left(B_{s, d} \rightarrow \mu^{+} \mu^{-}\right)$, Eur.Phys.J. C72 (2012) 2172, arXiv: 1208.0934].

[158] C. Bobeth, M. Gorbahn, and E. Stamou, Electroweak Corrections to

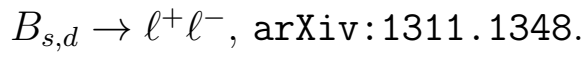

[159] T. Hermann, M. Misiak, and M. Steinhauser, Three-loop QCD corrections to $B_{s} \rightarrow \ell^{+} \ell^{-}$, arXiv: 1311.1347 .

[160] C. Bobeth, M. Gorbahn, T. Hermann, M. Misiak, E. Stamou, et. al., $B_{s, d} \rightarrow \ell^{+} \ell^{-}$in the Standard Model, arXiv:1311.0903.

[161] J. Brod, M. Gorbahn, and E. Stamou, Two-Loop Electroweak Corrections for the $K \rightarrow \pi \nu \bar{u} u$ Decays, Phys.Rev. D83 (2011) 034030, arXiv:1009.0947.

[162] LHCb collaboration Collaboration, R. Aaij et. al., Measurement of the $B_{s}^{0} \rightarrow \mu^{+} \mu^{-}$branching fraction and search for $B^{0} \rightarrow \mu^{+} \mu^{-}$decays at the $\mathrm{LHCb}$ experiment, Phys.Rev.Lett. 111 (2013) 101805, arXiv:1307.5024].

[163] CMS Collaboration Collaboration, S. Chatrchyan et. al., Measurement of the $B_{s} \rightarrow \mu \mu$ branching fraction and search for $B_{0} \rightarrow \mu \mu$ with the CMS Experiment, Phys.Rev.Lett. 111 (2013) 101804, arXiv:1307.5025].

[164] Combination of results on the rare decays $b \rightarrow \mu^{+} \mu^{-}$from the cms and lhcb experiments, Tech. Rep. CMS-PAS-BPH-13-007, CERN, Geneva, 2013.

[165] H. E. Logan and U. Nierste, $B_{s, d} \rightarrow \ell^{+} \ell^{-}$in a two Higgs doublet model, Nucl.Phys. B586 (2000) 39-55, hep-ph/0004139].

[166] W. Altmannshofer, M. Carena, N. R. Shah, and F. Yu, Indirect Probes of the MSSM after the Higgs Discovery, JHEP 1301 (2013) 160, arXiv:1211.1976. 
[167] A. J. Buras, B. Duling, and S. Gori, The Impact of Kaluza-Klein Fermions on Standard Model Fermion Couplings in a RS Model with Custodial Protection, JHEP 0909 (2009) 076, arXiv:0905.2318.

[168] F. del Aguila, J. de Blas, P. Langacker, and M. Perez-Victoria, Impact of extra particles on indirect Z' limits, Phys.Rev. D84 (2011) 015015, arXiv:1104.5512.

[169] F. Botella, G. Branco, and M. Nebot, The Hunt for New Physics in the Flavour Sector with up vector-like quarks, JHEP 1212 (2012) 040, arXiv:1207.4440.

[170] F. Beaujean, C. Bobeth, D. van Dyk, and C. Wacker, Bayesian Fit of Exclusive $b \rightarrow$ sele Decays: The Standard Model Operator Basis, JHEP 1208 (2012) 030, arXiv:1205.1838.

[171] J. Drobnak, S. Fajfer, and J. F. Kamenik, Probing anomalous tWb interactions with rare B decays, Nucl.Phys. B855 (2012) 82-99, arXiv:1109.2357].

[172] M. S. Chanowitz, The $Z \rightarrow \bar{b}$ b decay asymmetry and flavor changing neutral currents, hep-ph/9905478.

[173] U. Haisch and A. Weiler, Determining the Sign of the $Z$ Penguin Amplitude, Phys. Rev. D76 (2007) 074027, arXiv:0706.2054.

[174] A. Dighe, A. Kundu, and S. Nandi, Enhanced $B_{s}-\bar{B}_{s}$ lifetime difference and anomalous like-sign dimuon charge asymmetry from new physics in $B_{s} \rightarrow \tau^{+} \tau^{-}$, Phys.Rev. D82 (2010) 031502, arXiv:1005.4051.

[175] C. Bobeth and U. Haisch, New Physics in $\Gamma_{1} 2^{s}:(\bar{s} b)(\bar{\tau} \tau)$ Operators, Acta Phys.Polon. B44 (2013) 127-176, arXiv:1109.1826.

[176] BABAR Collaboration Collaboration, B. Aubert et. al., A Search for $B^{+} \rightarrow \tau^{+} \nu$ with Hadronic B tags, Phys.Rev. D77 (2008) 011107, arXiv:0708.2260.

[177] Belle Collaboration Collaboration, K. Ikado et. al., Evidence of the Purely Leptonic Decay $B^{-} \rightarrow \tau^{-} \bar{\nu}_{\tau}$, Phys.Rev.Lett. 97 (2006) 251802, hep-ex/0604018.

[178] Belle Collaboration Collaboration, I. Adachi et. al., Measurement of $B^{-} \rightarrow \tau^{-} \bar{\nu}_{\tau}$ with a Hadronic Tagging Method Using the Full Data Sample of Belle, Phys.Rev.Lett. 110 (2013) 131801, arXiv:1208.4678].

[179] UTfit Collaboration Collaboration, M. Bona et. al., An Improved Standard Model Prediction Of $B R(B \rightarrow \tau \nu)$ And Its Implications For New Physics, Phys.Lett. B687 (2010) 61-69, arXiv:0908.3470. 
[180] W.-S. Hou, Enhanced charged Higgs boson effects in $B^{-} \rightarrow \tau \bar{\nu}, \mu \bar{\nu}$ and $b \rightarrow$ taū $+X$, Phys.Rev. D48 (1993) 2342-2344.

[181] A. Akeroyd and S. Recksiegel, The Effect of $H^{ \pm}$on $B^{ \pm} \rightarrow \tau^{ \pm} \nu_{\tau}$ and $B^{ \pm} \rightarrow \mu^{ \pm} \nu_{\mu}$, J.Phys.G G29 (2003) 2311-2317, hep-ph/0306037].

[182] G. Isidori and P. Paradisi, Hints of large tan(beta) in flavour physics, Phys. Lett. B639 (2006) 499-507, [hep-ph/0605012].

[183] G. Blankenburg and G. Isidori, $B \rightarrow \tau \nu$ in two-Higgs doublet models with $M F V$, Eur.Phys.J.Plus 127 (2012) 85, arXiv:1107.1216.

[184] FlaviaNet Working Group on Kaon Decays Collaboration, M. Antonelli et. al., Precision tests of the Standard Model with leptonic and semileptonic kaon decays, arXiv:0801.1817. Updates available on http://www.lnf.infn.it/wg/vus/.

[185] U. Nierste, S. Trine, and S. Westhoff, Charged-Higgs effects in a new $B \rightarrow D \tau \nu$ differential decay distribution, Phys.Rev. D78 (2008) 015006, arXiv:0801.4938.

[186] BaBar Collaboration Collaboration, J. Lees et. al., Evidence for an excess of $\bar{B} \rightarrow D^{(*)} \tau^{-} \bar{\nu}_{\tau}$ decays, Phys.Rev.Lett. 109 (2012) 101802, [arXiv:1205.5442].

[187] S. Fajfer, J. F. Kamenik, and I. Nisandzic, On the $B \rightarrow D^{*} \tau \bar{\nu}_{\tau}$ Sensitivity to New Physics, Phys.Rev. D85 (2012) 094025, arXiv:1203.2654].

[188] J. F. Kamenik and F. Mescia, $B \rightarrow D \tau \nu$ Branching Ratios: Opportunity for Lattice QCD and Hadron Colliders, Phys.Rev. D78 (2008) 014003, arXiv:0802.3790.

[189] A. Crivellin, C. Greub, and A. Kokulu, Explaining $B \rightarrow D \tau \nu, B \rightarrow D^{*} \tau \nu$ and $B \rightarrow \tau \nu$ in a 2HDM of type III, Phys.Rev. D86 (2012) 054014, arXiv:1206.2634.

[190] A. Crivellin, A. Kokulu, and C. Greub, Flavor-phenomenology of two-Higgs-doublet models with generic Yukawa structure, Phys.Rev. D87 (2013) 094031, arXiv: 1303.5877.

[191] S. Fajfer, J. F. Kamenik, I. Nisandzic, and J. Zupan, Implications of Lepton Flavor Universality Violations in B Decays, Phys.Rev.Lett. 109 (2012) 161801, arXiv:1206.1872.

[192] P. Ko, Y. Omura, and C. Yu, $B \rightarrow D^{(*)} \tau \nu$ and $B \rightarrow \tau \nu$ in chiral U(1)' models with flavored multi Higgs doublets, JHEP 1303 (2013) 151, [arXiv:1212.4607].

[193] A. Crivellin, C. Greub, and A. Kokulu, Flavour-violation in two-Higgs-doublet models, arXiv:1309.4806. 
[194] M. Kaminski, M. Misiak, and M. Poradzinski, Tree-level contributions to $B \rightarrow X_{s} \gamma$, Phys.Rev. D86 (2012) 094004, arXiv:1209.0965].

[195] M. Misiak and M. Steinhauser, NNLO QCD corrections to the $\bar{B} \rightarrow X(s) \gamma$ matrix elements using interpolation in $m_{c}$, Nucl.Phys. B764 (2007) 62-82, hep-ph/0609241].

[196] M. Czakon, U. Haisch, and M. Misiak, Four-Loop Anomalous Dimensions for Radiative Flavour-Changing Decays, JHEP 0703 (2007) 008, hep-ph/0612329].

[197] R. Boughezal, M. Czakon, and T. Schutzmeier, NNLO fermionic corrections to the charm quark mass dependent matrix elements in $\bar{B} \rightarrow X_{s} \gamma$, JHEP 0709 (2007) 072, arXiv:0707.3090].

[198] H. Asatrian, T. Ewerth, H. Gabrielyan, and C. Greub, Charm quark mass dependence of the electromagnetic dipole operator contribution to $\bar{B} \rightarrow X_{s} \gamma$ at $O\left(\alpha_{s}^{2}\right)$, Phys.Lett. B647 (2007) 173-178, hep-ph/0611123.

[199] T. Ewerth, Fermionic corrections to the interference of the electro- and chromomagnetic dipole operators in $\bar{B} \rightarrow X_{s} \gamma$ at $O\left(\alpha_{s}^{2}\right)$, Phys.Lett. B669 (2008) 167-172, arXiv:0805.3911.

[200] H. Asatrian, T. Ewerth, A. Ferroglia, C. Greub, and G. Ossola, Complete $\left(O_{7}, O_{8}\right)$ contribution to $B \rightarrow X_{s} \gamma$ at order $\alpha_{s}^{2}$, Phys.Rev. D82 (2010) 074006, arXiv: 1005.5587.

[201] A. Ferroglia and U. Haisch, Chromomagnetic Dipole-Operator Corrections in $\bar{B} \rightarrow X_{s \gamma}$ at $O\left(\beta_{0} \alpha_{s}^{2}\right)$, Phys.Rev. D82 (2010) 094012, arXiv:1009.2144.

[202] M. Misiak and M. Poradzinski, Completing the Calculation of BLM corrections to $\bar{B} \rightarrow X_{s} \gamma$, Phys.Rev. D83 (2011) 014024, arXiv:1009.5685].

[203] M. Czakon, P. Fiedler, T. Huber, M. Misiak, T. Schutzmeier, and M. Steinhauser, "to be published." 2013.

[204] J. M. Soares, CP violation in radiative b decays, Nucl.Phys. B367 (1991) $575-590$.

[205] A. L. Kagan and M. Neubert, Direct CP violation in $B \rightarrow X_{s} \gamma$ decays as a signature of new physics, Phys.Rev. D58 (1998) 094012, hep-ph/9803368.

[206] A. L. Kagan and M. Neubert, QCD anatomy of $B \rightarrow X_{s} \gamma$ decays, Eur.Phys.J. C7 (1999) 5-27, hep-ph/9805303.

[207] T. Hurth, E. Lunghi, and W. Porod, Untagged $\bar{B} \rightarrow X_{s+d} \gamma$ CP asymmetry as a probe for new physics, Nucl.Phys. B704 (2005) 56-74, [hep-ph/0312260]. 
[208] M. Benzke, S. J. Lee, M. Neubert, and G. Paz, Long-Distance Dominance of the CP Asymmetry in $B \rightarrow X_{s, d} \gamma$ Decays, Phys.Rev.Lett. 106 (2011) 141801, arXiv:1012.3167.

[209] S. W. Bosch and G. Buchalla, The Radiative decays $B \rightarrow V \gamma$ at next-to-leading order in QCD, Nucl.Phys. B621 (2002) 459-478, hep-ph/0106081.

[210] S. W. Bosch and G. Buchalla, Constraining the unitarity triangle with $B \rightarrow V \gamma$ , JHEP 0501 (2005) 035, hep-ph/0408231.

[211] D. Atwood, M. Gronau, and A. Soni, Mixing induced CP asymmetries in radiative $B$ decays in and beyond the standard model, Phys.Rev.Lett. 79 (1997) 185-188, hep-ph/9704272.

[212] P. Ball and R. Zwicky, Time-dependent CP Asymmetry in $B \rightarrow K^{*} \gamma$ as a (Quasi) Null Test of the Standard Model, Phys.Lett. B642 (2006) 478-486, hep-ph/0609037.

[213] P. Ball, G. W. Jones, and R. Zwicky, $B \rightarrow V \gamma$ beyond QCD factorisation, Phys.Rev. D75 (2007) 054004, hep-ph/0612081.

[214] Belle Collaboration Collaboration, Y. Ushiroda et. al., Time-Dependent CP Asymmetries in $B^{0} \rightarrow K_{s}^{0} \pi^{0} \gamma$ transitions, Phys.Rev. D74 (2006) 111104, hep-ex/0608017.

[215] BABAR Collaboration Collaboration, B. Aubert et. al., Measurement of Time-Dependent CP Asymmetry in $B^{0} \rightarrow K_{S}^{0} \pi^{0} \gamma$ Decays, Phys.Rev. D78 (2008) 071102, arXiv:0807.3103.

[216] Heavy Flavor Averaging Group Collaboration, D. Asner et. al., Averages of b-hadron, c-hadron, and $\tau$-lepton Properties, arXiv:1010.1589. Long author list - awaiting processing.

[217] J. Lyon and R. Zwicky, Isospin asymmetries in $B \rightarrow\left(K^{*}, \rho\right) \gamma / l^{+} l^{-}$and $B \rightarrow \mathrm{Kl}^{+} \mathrm{l}^{-}$in and beyond the Standard Model, Phys.Rev. D88 (2013) 094004, arXiv: 1305.4797.

[218] U. Haisch, $\bar{B} \rightarrow X_{s} \gamma$ : Standard Model and Beyond, arXiv:0805.2141.

[219] B. Grzadkowski and M. Misiak, Anomalous Wtb coupling effects in the weak radiative B-meson decay, Phys.Rev. D78 (2008) 077501, arXiv:0802.1413.

[220] W. Altmannshofer, D. Guadagnoli, S. Raby, and D. M. Straub, SUSY GUTs with Yukawa unification: A Go/no-go study using FCNC processes, Phys. Lett. B668 (2008) 385-391, arXiv:0801.4363.

[221] K. Agashe, N. G. Deshpande, and G. H. Wu, Universal extra dimensions and $b \rightarrow s \gamma$, Phys. Lett. B514 (2001) 309-314, hep-ph/0105084. 
[222] A. J. Buras, A. Poschenrieder, M. Spranger, and A. Weiler, The impact of universal extra dimensions on $b \rightarrow x_{s} \gamma, b \rightarrow x_{s}$ gluon, $b \rightarrow x_{s} \mu^{+} \mu^{-}$, $k_{l} \rightarrow \pi^{0} e^{+} e^{-}$, and $\varepsilon^{\prime} / \varepsilon$, Nucl. Phys. B678 (2004) 455-490, hep-ph/0306158.

[223] U. Haisch and A. Weiler, Bound on minimal universal extra dimensions from $\bar{B} \rightarrow X_{s} \gamma$, Phys.Rev. D76 (2007) 034014, hep-ph/0703064.

[224] A. Freitas and U. Haisch, $\bar{B} \rightarrow X_{s} \gamma$ in two universal extra dimensions, Phys.Rev. D77 (2008) 093008, arXiv:0801.4346.

[225] G. Asatrian and A. Ionnisian, RARE B MESON DECAYS IN $S U(2)_{L} \times S U(2)_{R} \times U(1)$ MODEL, Mod.Phys.Lett. A5 (1990) 1089-1096.

[226] G. Asatryan and A. Ioannisyan, The $b \rightarrow s \gamma$ decay in $S U(2)_{L} \times S U(2)_{R} \times U(1)$ model. (In Russian), Sov.J.Nucl.Phys. 51 (1990) 858-860.

[227] D. Cocolicchio, G. Costa, G. L. Fogli, J. Kim, and A. Masiero, RARE B DECAYS IN LEFT-RIGHT SYMMETRIC MODELS, Phys.Rev. D40 (1989) 1477.

[228] P. L. Cho and M. Misiak, $b \rightarrow s \gamma$ decay in $S U(2)_{L} \times S U(2)_{R} \times U(1)$ extensions of the Standard Model, Phys.Rev. D49 (1994) 5894-5903, hep-ph/9310332.

[229] K. Babu, K. Fujikawa, and A. Yamada, Constraints on left-right symmetric models from the process $b \rightarrow s \gamma$, Phys.Lett. B333 (1994) 196-201, hep-ph/9312315.

[230] K. Fujikawa and A. Yamada, Test of the chiral structure of the top - bottom charged current by the process $b \rightarrow s \gamma$, Phys.Rev. D49 (1994) 5890-5893.

[231] G. Asatrian and A. Ioannisian, CP violation in the decay $b \rightarrow s \gamma$ in the left-right symmetric model, Phys.Rev. D54 (1996) 5642-5646, hep-ph/9603318.

[232] C. Bobeth, M. Misiak, and J. Urban, Matching conditions for $b \rightarrow s \gamma$ and $b \rightarrow s g$ in extensions of the standard model, Nucl.Phys. B567 (2000) 153-185, hep-ph/9904413.

[233] M. Frank, A. Hayreter, and I. Turan, B Decays in an Asymmetric Left-Right Model, Phys.Rev. D82 (2010) 033012, arXiv:1005.3074].

[234] D. Guadagnoli, R. N. Mohapatra, and I. Sung, Gauged Flavor Group with Left-Right Symmetry, JHEP 1104 (2011) 093, arXiv:1103.4170.

[235] C. Bobeth, G. Hiller, and G. Piranishvili, CP Asymmetries in bar $B \rightarrow \bar{K}^{*}(\rightarrow \bar{K} \pi) \bar{\ell} \ell$ and Untagged $\bar{B}_{s}, B_{s} \rightarrow \phi\left(\rightarrow K^{+} K^{-}\right) \bar{\ell} \ell$ Decays at $N L O$, JHEP 0807 (2008) 106, arXiv:0805.2525. 
[236] U. Egede, T. Hurth, J. Matias, M. Ramon, and W. Reece, New observables in the decay mode $\bar{B}_{d} \rightarrow \bar{K}^{* 0} l^{+} l^{-}$, JHEP 0811 (2008) 032, arXiv:0807.2589.

[237] W. Altmannshofer, P. Ball, A. Bharucha, A. J. Buras, D. M. Straub, et. al., Symmetries and Asymmetries of $B \rightarrow K^{*} \mu^{+} \mu^{-}$Decays in the Standard Model and Beyond, JHEP 0901 (2009) 019, arXiv:0811.1214.

[238] C. Bobeth, G. Hiller, and D. van Dyk, The Benefits of $\bar{B} \rightarrow \bar{K}^{*} l^{+} l^{-}$Decays at Low Recoil, JHEP 1007 (2010) 098, arXiv:1006.5013.

[239] D. Becirevic, N. Kosnik, F. Mescia, and E. Schneider, Complementarity of the constraints on New Physics from $B_{s} \rightarrow \mu^{+} \mu^{-}$and from $B \rightarrow K \ell^{+} \ell^{-}$decays, Phys.Rev. D86 (2012) 034034, arXiv:1205.5811.

[240] C. Bobeth, G. Hiller, and D. van Dyk, More Benefits of Semileptonic Rare B Decays at Low Recoil: CP Violation, JHEP 1107 (2011) 067, arXiv:1105.0376.

[241] S. Descotes-Genon, J. Matias, M. Ramon, and J. Virto, Implications from clean observables for the binned analysis of $B \rightarrow K * \mu^{+} \mu^{-}$at large recoil, JHEP 1301 (2013) 048, arXiv:1207.2753.

[242] S. Jager and J. M. Camalich, On B $\rightarrow$ Vle at small dilepton invariant mass, power corrections, and new physics, JHEP 1305 (2013) 043, arXiv:1212.2263.

[243] BELLE Collaboration Collaboration, J.-T. Wei et. al., Measurement of the Differential Branching Fraction and Forward-Backword Asymmetry for $B \rightarrow K^{(*)} l^{+} l^{-}$, Phys.Rev.Lett. 103 (2009) 171801, arXiv:0904.0770].

[244] CDF Collaboration Collaboration, T. Aaltonen et. al., Measurements of the Angular Distributions in the Decays $B \rightarrow K^{(*)} \mu^{+} \mu^{-}$at CDF, Phys.Rev.Lett. 108 (2012) 081807, arXiv:1108.0695.

[245] BaBar Collaboration Collaboration, J. Lees et. al., Measurement of Branching Fractions and Rate Asymmetries in the Rare Decays $B \rightarrow K^{(*)} l^{+} l^{-}$, Phys.Rev. D86 (2012) 032012, arXiv:1204.3933.

[246] LHCb Collaboration Collaboration, R. Aaij et. al., Differential branching fraction and angular analysis of the decay $B^{0} \rightarrow K^{* 0} \mu^{+} \mu^{-}$, Phys.Rev.Lett. 108 (2012) 181806, arXiv:1112.3515.

[247] LHCb Collaboration Collaboration, R. Aaij et. al., Differential branching fraction and angular analysis of the decay $B^{0} \rightarrow K^{* 0} \mu^{+} \mu^{-}$, JHEP 1308 (2013) 131, arXiv:1304.6325.

[248] S. Descotes-Genon, T. Hurth, J. Matias, and J. Virto, Optimizing the basis of $B \rightarrow K^{*} \ell^{+} \ell^{-}$observables in the full kinematic range, JHEP 1305 (2013) 137, arXiv:1303.5794. 
[249] S. Descotes-Genon, T. Hurth, J. Matias, and J. Virto, $B \rightarrow K^{*} \ell \ell$ : The New Frontier of New Physics searches in Flavor, arXiv:1305.4808.

[250] F. Kruger and J. Matias, Probing new physics via the transverse amplitudes of $B^{0} \rightarrow K * 0\left(K^{-} \pi^{+}\right) l^{+} l^{-}$at large recoil, Phys.Rev. D71 (2005) 094009, hep-ph/0502060.

[251] U. Egede, T. Hurth, J. Matias, M. Ramon, and W. Reece, New physics reach of the decay mode $\bar{B} \rightarrow \bar{K}^{* 0} \ell^{+} \ell^{-}$, JHEP 1010 (2010) 056, arXiv:1005.0571].

[252] D. Becirevic and E. Schneider, On transverse asymmetries in $B \rightarrow K^{*} l^{+} l^{-}$, Nucl.Phys. B854 (2012) 321-339, arXiv:1106.3283].

[253] C. Bobeth, G. Hiller, and D. van Dyk, General Analysis of $\bar{B} \rightarrow \bar{K}^{(*)} \ell^{+} \ell^{-}$ Decays at Low Recoil, Phys.Rev. D87 (2013) 034016, arXiv:1212.2321.

[254] J. Matias, F. Mescia, M. Ramon, and J. Virto, Complete Anatomy of $\bar{B}_{d} \rightarrow \bar{K}^{* 0}(\rightarrow ; K \pi) l^{+} l^{-}$and its angular distribution, JHEP 1204 (2012) 104, arXiv:1202.4266.

[255] S. Descotes-Genon, J. Matias, and J. Virto, Understanding the $B \rightarrow K^{*} \mu^{+} \mu^{-}$ Anomaly, Phys. Rev. D 88, 074002 (2013) arXiv:1307.5683.

[256] LHCb collaboration Collaboration, R. Aaij et. al., Measurement of form-factor independent observables in the decay $B^{0} \rightarrow K^{* 0} \mu^{+} \mu^{-}$, Phys.Rev.Lett. 111 (2013) 191801, arXiv:1308.1707.

[257] W. Altmannshofer and D. M. Straub, New physics in $B \rightarrow K^{*} \mu \mu$ ?, arXiv:1308.1501.

[258] LHCb Collaboration Collaboration, R. Aaij et. al., Measurement of the isospin asymmetry in $B \rightarrow K^{(*)} \mu^{+} \mu^{-}$decays, JHEP 1207 (2012) 133, arXiv:1205.3422.

[259] M. Beylich, G. Buchalla, and T. Feldmann, Theory of $B \rightarrow K^{(*)} l^{+} l^{-}$decays at high $q^{2}$ : OPE and quark-hadron duality, Eur.Phys.J. C71 (2011) 1635, arXiv:1101.5118.

[260] R. R. Horgan, Z. Liu, S. Meinel, and M. Wingate, Lattice QCD calculation of form factors describing the rare decays $B \rightarrow K^{*} \ell^{+} \ell^{-}$and $B_{s} \rightarrow \phi \ell^{+} \ell^{-}$, Phys.Rev. D89 (2014) 094501, arXiv:1310.3722.

[261] C. Bouchard, G. P. Lepage, C. Monahan, H. Na, and J. Shigemitsu, Standard Model predictions for $B \rightarrow$ Kll with form factors from lattice QCD, Phys. Rev. Lett. 111, 162002 (2013) arXiv:1306.0434. 
[262] C. Bouchard, G. P. Lepage, C. Monahan, H. Na, and J. Shigemitsu, Rare decay $B \rightarrow$ Kll form factors from lattice QCD, Phys. Rev. D 88, 054509 (2013) 054509, arXiv:1306.2384.

[263] C. Bobeth, M. Misiak, and J. Urban, Photonic penguins at two loops and $m_{t^{-}}$-dependence of $B R\left(B \rightarrow X_{s} \ell^{+} \ell^{-}\right)$, Nucl. Phys. B574 (2000) 291-330, hep-ph/9910220.

[264] H. Asatrian, H. Asatrian, C. Greub, and M. Walker, Two loop virtual corrections to $B \rightarrow X(s) l^{+} l^{-}$in the standard model, Phys.Lett. B507 (2001) 162-172, hep-ph/0103087.

[265] H. H. Asatryan, H. M. Asatrian, C. Greub, and M. Walker, Calculation of two loop virtual corrections to $b \rightarrow s l^{+} l^{-}$in the standard model, Phys. Rev. D65 (2002) 074004, hep-ph/0109140|.

[266] A. Ghinculov, T. Hurth, G. Isidori, and Y. P. Yao, The rare decay $B-\dot{\delta} X / s l+$ l- to NNLL precision for arbitrary dilepton invariant mass, Nucl. Phys. B685 (2004) 351-392, hep-ph/0312128.

[267] Z. Ligeti and F. J. Tackmann, Precise predictions for $B \rightarrow X(s) l^{+} l^{-}$in the large $q^{* * 2}$ region, Phys.Lett. B653 (2007) 404-410, arXiv:0707.1694].

[268] C. Greub, V. Pilipp, and C. Schupbach, Analytic calculation of two-loop QCD corrections to $b \rightarrow s l^{+} l^{-}$in the high $q^{* * 2}$ region, JHEP 0812 (2008) 040, arXiv:0810.4077.

[269] S. Descotes-Genon, D. Ghosh, J. Matias, and M. Ramon, Exploring New Physics in the $C_{7}-C_{7}^{\prime}$ plane, JHEP 1106 (2011) 099, arXiv:1104.3342].

[270] C. Hambrock, G. Hiller, S. Schacht, and R. Zwicky, $B \rightarrow K^{*}$ Form Factors from Flavor Data to QCD and Back, arXiv:1308.4379.

[271] R. Barbieri, P. Lodone, and D. M. Straub, CP Violation in Supersymmetry with Effective Minimal Flavour Violation, JHEP 1105 (2011) 049, arXiv:1102.0726.

[272] CMS Collaboration Collaboration, S. Chatrchyan et. al., Angular analysis and branching fraction measurement of the decay $B^{0} \rightarrow K^{* 0} \mu^{+} \mu^{-}$, Phys.Lett. B727 (2013) 77-100, arXiv:1308.3409].

[273] R. Gauld, F. Goertz, and U. Haisch, On minimal $Z^{\prime}$ explanations of the $B \rightarrow K^{*} \mu^{+} \mu^{-}$anomaly, arXiv:1308.1959.

[274] R. Gauld, F. Goertz, and U. Haisch, An explicit Z'-boson explanation of the $B \rightarrow K^{*} \mu^{+} \mu^{-}$anomaly, JHEP 1401 (2014) 069, arXiv:1310.1082. 
[275] F. Beaujean, C. Bobeth, and D. van Dyk, Comprehensive Bayesian Analysis of Rare (Semi)leptonic and Radiative B Decays, arXiv:1310.2478.

[276] A. Datta, M. Duraisamy, and D. Ghosh, Explaining the $B \rightarrow K^{*} \mu^{+} \mu^{-}$anomaly with scalar interactions, arXiv:1310.1937.

[277] R. R. Horgan, Z. Liu, S. Meinel, and M. Wingate, Calculation of $B^{0} \rightarrow K^{* 0} \mu^{+} \mu^{-}$and $B_{s}^{0} \rightarrow \phi \mu^{+} \mu^{-}$observables using form factors from lattice $Q C D$, arXiv:1310.3887.

[278] F. Richard, $A Z^{\prime}$ interpretation of $B_{d} \rightarrow K^{*} \mu^{+} \mu^{-}$data and consequences for high energy colliders, arXiv:1312.2467.

[279] T. Hurth and F. Mahmoudi, On the LHCb anomaly in $B \rightarrow K^{*} l^{+} l^{-}$, arXiv:1312.5267.

[280] A. Khodjamirian, T. Mannel, A. Pivovarov, and Y.-M. Wang, Charm-loop effect in $B \rightarrow K^{(*)} \ell^{+} \ell^{-}$and $B \rightarrow K^{*} \gamma$, JHEP 1009 (2010) 089, arXiv:1006.4945.

[281] J. Matias, On the S-wave pollution of $B \rightarrow K * l^{+} l$ - observables, Phys.Rev. D86 (2012) 094024, arXiv:1209.1525.

[282] D. M. Straub, Constraints on new physics from rare (semi-)leptonic B decays, arXiv: 1305.5704.

[283] A. J. Buras, M. Gorbahn, U. Haisch, and U. Nierste, The rare decay $K^{+} \rightarrow \pi^{+} \nu \bar{\nu}$ at the next-to-next-to-leading order in QCD, Phys. Rev. Lett. 95 (2005) 261805, hep-ph/0508165.

[284] A. J. Buras, M. Gorbahn, U. Haisch, and U. Nierste, Charm quark contribution to $K^{+} \rightarrow \pi^{+} \nu \bar{\nu}$ at next-to-next-to-leading order, JHEP 11 (2006) 002, hep-ph/0603079.

[285] J. Brod and M. Gorbahn, Electroweak Corrections to the Charm Quark Contribution to $K^{+} \rightarrow \pi^{+} \nu \bar{\nu}$, Phys. Rev. D78 (2008) 034006, arXiv:0805.4119.

[286] G. Isidori, F. Mescia, and C. Smith, Light-quark loops in $K \rightarrow \pi \nu \bar{\nu}$, Nucl. Phys. B718 (2005) 319-338, [hep-ph/0503107].

[287] F. Mescia and C. Smith, Improved estimates of rare $K$ decay matrix-elements from $K_{\ell 3}$ decays, Phys. Rev. D76 (2007) 034017, arXiv:0705.2025].

[288] A. J. Buras, F. Schwab, and S. Uhlig, Waiting for precise measurements of $K^{+} \rightarrow \pi^{+} \nu \bar{\nu}$ and $K_{L} \rightarrow \pi^{0} \nu \bar{\nu}$, Rev. Mod. Phys. 80 (2008) 965-1007, hep-ph/0405132. 
[289] G. Isidori, Flavor Physics with light quarks and leptons, eConf C060409 (2006) 035, hep-ph/0606047].

[290] C. Smith, Theory review on rare K decays: Standard model and beyond, hep-ph/0608343.

[291] T. Komatsubara, Experiments with K-Meson Decays, Prog.Part.Nucl.Phys. 67 (2012) 995-1018, arXiv:1203.6437.

[292] M. Blanke, New Physics Signatures in Kaon Decays, PoS KAON13 (2013) 010, arXiv:1305.5671.

[293] A. J. Buras, R. Fleischer, S. Recksiegel, and F. Schwab, Anatomy of prominent $B$ and $K$ decays and signatures of $C P$-violating new physics in the electroweak penguin sector, Nucl. Phys. B697 (2004) 133-206, hep-ph/0402112].

[294] A. J. Buras, K. Gemmler, and G. Isidori, Quark flavour mixing with right-handed currents: an effective theory approach, Nucl.Phys. B843 (2011) 107-142, arXiv: 1007.1993].

[295] E949 Collaboration, A. V. Artamonov et. al., New measurement of the $K^{+} \rightarrow \pi^{+} \nu \bar{\nu}$ branching ratio, Phys. Rev. Lett. 101 (2008) 191802, arXiv:0808.2459.

[296] E391a Collaboration Collaboration, J. Ahn et. al., Experimental study of the decay $K_{L}^{0} \rightarrow \pi^{0} \nu \bar{\nu}$, Phys.Rev. D81 (2010) 072004, arXiv:0911.4789.

[297] ORKA Collaboration, E. Worcester, ORKA, The Golden Kaon Experiment: Precision measurement of $K^{+} \rightarrow \pi^{+} \nu \bar{\nu}$ and other rare processes, PoS KAON13 (2013) 035, arXiv:1305.7245].

[298] G. Buchalla, G. D'Ambrosio, and G. Isidori, Extracting short-distance physics from $K_{L, S} \rightarrow \pi^{0} e^{+} e^{-}$decays, Nucl. Phys. B672 (2003) 387-408, hep-ph/0308008.

[299] G. Isidori, C. Smith, and R. Unterdorfer, The rare decay $K_{L} \rightarrow \pi^{0} \mu^{+} \mu^{-}$within the SM, Eur. Phys. J. C36 (2004) 57-66, hep-ph/0404127].

[300] S. Friot, D. Greynat, and E. De Rafael, Rare kaon decays revisited, Phys. Lett. B595 (2004) 301-308, [hep-ph/0404136].

[301] F. Mescia, C. Smith, and S. Trine, $K_{L} \rightarrow \pi^{0} e^{+} e^{-}$and $K_{L} \rightarrow \pi^{0} \mu^{+} \mu^{-}:$A binary star on the stage of flavor physics, JHEP 08 (2006) 088, [hep-ph/0606081].

[302] J. Prades, ChPT Progress on Non-Leptonic and Radiative Kaon Decays, PoS KAON (2008) 022, arXiv:0707.1789. 
[303] A. J. Buras, M. E. Lautenbacher, M. Misiak, and M. Munz, Direct CP violation in $K_{L} \rightarrow \pi^{0} e^{+} e^{-}$beyond leading logarithms, Nucl. Phys. B423 (1994) 349-383, hep-ph/9402347.

[304] M. Gorbahn and U. Haisch, Charm quark contribution to $K_{L} \rightarrow \mu^{+} \mu^{-}$at next-to-next-to-leading order, Phys. Rev. Lett. 97 (2006) 122002, hep-ph/0605203.

[305] G. Isidori and R. Unterdorfer, On the short-distance constraints from $K_{L, S} \rightarrow \mu^{+} \mu^{-}$, JHEP 01 (2004) 009, hep-ph/0311084.

[306] Y. Grossman and Y. Nir, $K_{L} \rightarrow \pi^{0} \nu \bar{\nu}$ beyond the standard model, Phys. Lett. B398 (1997) 163-168, hep-ph/9701313.

[307] M. Blanke, A. J. Buras, B. Duling, S. Recksiegel, and C. Tarantino, FCNC Processes in the Littlest Higgs Model with T-Parity: a 2009 Look, Acta Phys.Polon. B41 (2010) 657-683, arXiv:0906.5454.

[308] P. Colangelo, F. De Fazio, P. Santorelli, and E. Scrimieri, Rare $B \rightarrow K^{(*)} \nu \bar{\nu}$ decays at $B$ factories, Phys.Lett. B395 (1997) 339-344, hep-ph/9610297].

[309] G. Buchalla, G. Hiller, and G. Isidori, Phenomenology of non-standard Z couplings in exclusive semileptonic $b \rightarrow$ s transitions, Phys. Rev. D63 (2001) 014015, hep-ph/0006136.

[310] W. Altmannshofer, A. J. Buras, D. M. Straub, and M. Wick, New strategies for New Physics search in $B \rightarrow K^{*} \nu \bar{\nu}, B \rightarrow K \nu \bar{\nu}$ and $B \rightarrow X_{s} \nu \bar{\nu}$ decays, JHEP 04 (2009) 022, arXiv:0902.0160.

[311] M. Bartsch, M. Beylich, G. Buchalla, and D.-N. Gao, Precision Flavour Physics with $B \rightarrow K \nu \bar{\nu}$ and $B \rightarrow K l^{+} l^{-}$, JHEP 0911 (2009) 011, arXiv:0909.1512.

[312] J. F. Kamenik and C. Smith, Tree-level contributions to the rare decays $B^{+} \rightarrow \pi^{+} \nu \bar{\nu}, B^{+} \rightarrow K^{+} \nu \bar{\nu}$, and $B^{+} \rightarrow K^{*+} \nu \bar{\nu}$ in the Standard Model, Phys.Lett. B680 (2009) 471-475, arXiv:0908.1174.

[313] ALEPH Collaboration, R. Barate et. al., Measurements of $B R\left(b \rightarrow \tau^{-} \bar{\nu}_{\tau} X\right)$ and $B R\left(b \rightarrow \tau^{-} \bar{\nu}_{\tau} D^{* \pm} X\right)$ and upper limits on $B R\left(B^{-} \rightarrow \tau^{-} \bar{\nu}_{\tau}\right)$ and $B R(b \rightarrow \nu \bar{\nu})$, Eur. Phys. J. C19 (2001) 213-227, hep-ex/0010022.

[314] BELLE Collaboration, K. F. Chen et. al., Search for $B \rightarrow h^{(*)} \nu \bar{\nu}$ Decays at Belle, Phys. Rev. Lett. 99 (2007) 221802, arXiv:0707.0138.

[315] BABAR Collaboration, B. Aubert et. al., Search for $B \rightarrow K^{*} \nu \bar{\nu}$ decays, Phys. Rev. D78 (2008) 072007, arXiv:0808.1338. 
[316] T. Blum, P. Boyle, N. Christ, N. Garron, E. Goode, et. al., The $K \rightarrow(\pi \pi)_{I=2}$ Decay Amplitude from Lattice QCD, Phys.Rev.Lett. 108 (2012) 141601, arXiv:1111.1699.

[317] RBC Collaboration, UKQCD Collaboration Collaboration, N. H. Christ, Theoretical strategies for $\epsilon^{\prime} / \epsilon$, PoS KAON09 (2009) 027, arXiv:0912.2917.

[318] A. J. Buras, M. Jamin, and M. E. Lautenbacher, The anatomy of $\varepsilon^{\prime} / \varepsilon$ beyond leading logarithms with improved hadronic matrix elements, Nucl. Phys. B408 (1993) 209-285, hep-ph/9303284.

[319] M. Ciuchini, E. Franco, G. Martinelli, and L. Reina, The $\Delta S=1$ effective Hamiltonian including next-to-leading order $Q C D$ and QED corrections, Nucl.Phys. B415 (1994) 403-462, hep-ph/9304257.

[320] A. J. Buras, P. Gambino, and U. A. Haisch, Electroweak penguin contributions to non-leptonic $\delta f=1$ decays at nnlo, Nucl. Phys. B570 (2000) 117-154, hep-ph/9911250].

[321] M. Gorbahn and U. Haisch, Effective Hamiltonian for non-leptonic $|\Delta F|=1$ decays at NNLO in QCD, Nucl.Phys. B713 (2005) 291-332, hep-ph/0411071.

[322] NA48 Collaboration Collaboration, J. Batley et. al., A Precision measurement of direct $C P$ violation in the decay of neutral kaons into two pions, Phys.Lett. B544 (2002) 97-112, hep-ex/0208009].

[323] KTeV Collaboration Collaboration, A. Alavi-Harati et. al., Measurements of direct CP violation, CPT symmetry, and other parameters in the neutral kaon system, Phys.Rev. D67 (2003) 012005, hep-ex/0208007].

[324] KTeV Collaboration Collaboration, E. Worcester, The Final Measurement of $\varepsilon^{\prime} / \varepsilon$ from $K T e V$, arXiv:0909.2555.

[325] M. Blanke, A. J. Buras, S. Recksiegel, C. Tarantino, and S. Uhlig, Correlations between $\varepsilon^{\prime} / \varepsilon$ and Rare $K$ Decays in the Littlest Higgs Model with T-Parity, JHEP 06 (2007) 082, 0704.3329.

[326] W. A. Bardeen, A. J. Buras, and J.-M. Gérard, The $K \rightarrow \pi \pi$ Decays in the Large N Limit: Quark Evolution, Nucl.Phys. B293 (1987) 787.

[327] S. Bertolini, M. Fabbrichesi, and J. O. Eeg, Theory of the CP violating parameter $\epsilon^{\prime} / \epsilon$, Rev.Mod.Phys. 72 (2000) 65-93, hep-ph/9802405].

[328] A. J. Buras and M. Jamin, $\varepsilon^{\prime} / \varepsilon$ at the nlo: 10 years later, JHEP 01 (2004) 048, hep-ph/0306217.

[329] A. Pich, $\varepsilon^{\prime} / \varepsilon$ in the standard model: Theoretical update, hep-ph/0410215. 
[330] V. Cirigliano, G. Ecker, H. Neufeld, A. Pich, and J. Portoles, Kaon Decays in the Standard Model, Rev.Mod.Phys. 84 (2012) 399, arXiv:1107.6001.

[331] S. Bertolini, J. O. Eeg, A. Maiezza, and F. Nesti, New physics in $\epsilon^{\prime}$ from gluomagnetic contributions and limits on Left-Right symmetry, Phys.Rev. D86 (2012) 095013, arXiv:1206.0668.

[332] J. M. Flynn and L. Randall, The Electromagnetic Penguin Contribution to $\varepsilon^{\prime} / \varepsilon$ for Large Top Quark Mass, Phys.Lett. B224 (1989) 221.

[333] G. Buchalla, A. J. Buras, and M. K. Harlander, The anatomy of $\varepsilon^{\prime} / \varepsilon$ in the standard model, Nucl. Phys. B337 (1990) 313-362.

[334] T. Blum, P. Boyle, N. Christ, N. Garron, E. Goode, et. al., $K \rightarrow \pi \pi$ Decay amplitudes from Lattice QCD, Phys.Rev. D84 (2011) 114503, arXiv:1106.2714.

[335] T. Blum, P. Boyle, N. Christ, N. Garron, E. Goode, et. al., Lattice determination of the $K \rightarrow(\pi \pi)_{I=2}$ Decay Amplitude $A_{2}$, Phys.Rev. D86 (2012) 074513, arXiv:1206.5142.

[336] LHCb Collaboration Collaboration, R. Aaij et. al., Evidence for CP violation in time-integrated $D^{0} \rightarrow h^{-} h^{+}$decay rates, Phys.Rev.Lett. 108 (2012) 111602, arXiv:1112.0938.

[337] LHCb collaboration Collaboration, R. Aaij et. al., Search for direct CP violation in $D^{0} \rightarrow h^{-} h^{+}$modes using semileptonic $B$ decays, Phys.Lett. B723 (2013) 33-43, $\operatorname{arXiv:1303.2614\text {.}}$

[338] G. Isidori, J. F. Kamenik, Z. Ligeti, and G. Perez, Implications of the LHCb Evidence for Charm CP Violation, Phys.Lett. B711 (2012) 46-51, arXiv:1111.4987.

[339] Y. Hochberg and Y. Nir, Relating direct CP violation in D decays and the forward-backward asymmetry in $t \bar{t}$ production, Phys.Rev.Lett. 108 (2012) 261601, $\operatorname{arXiv:1112.5268\text {.}}$

[340] G. Isidori and J. F. Kamenik, Shedding light on CP violation in the charm system via $D \rightarrow V \gamma$ decays, Phys.Rev.Lett. 109 (2012) 171801, arXiv:1205.3164.

[341] K. Blum, Y. Grossman, Y. Nir, and G. Perez, Combining $K^{0}-\bar{K}^{0}$ mixing and $D^{0}-\bar{D}^{0}$ mixing to constrain the flavor structure of new physics, Phys.Rev.Lett. 102 (2009) 211802, arXiv:0903.2118.

[342] O. Gedalia, J. F. Kamenik, Z. Ligeti, and G. Perez, On the Universality of CP Violation in $\Delta F=1$ Processes, Phys.Lett. B714 (2012) 55-61, arXiv:1202.5038. 
[343] A. J. Buras, G. Perez, T. A. Schwarz, and T. M. Tait, Top and flavour physics in the LHC era, Eur.Phys.J. C72 (2012) 2105.

[344] F. Jegerlehner and A. Nyffeler, The Muon g-2, Phys. Rept. 477 (2009) 1-110, arXiv:0902.3360.

[345] J. Engel, M. J. Ramsey-Musolf, and U. van Kolck, Electric Dipole Moments of Nucleons, Nuclei, and Atoms: The Standard Model and Beyond, Prog.Part.Nucl.Phys. 71 (2013) 21-74, arXiv:1303.2371].

[346] R. H. Bernstein and P. S. Cooper, Charged Lepton Flavor Violation: An Experimenter's Guide, Phys.Rept. (2013) arXiv:1307.5787.

[347] J. Hisano, M. Nagai, P. Paradisi, and Y. Shimizu, Waiting for $\mu \rightarrow e \gamma$ from the MEG experiment, JHEP 0912 (2009) 030, [arXiv:0904.2080].

[348] A. J. Buras, M. Nagai, and P. Paradisi, Footprints of SUSY GUTs in Flavour Physics, JHEP 1105 (2011) 005, arXiv:1011.4853.

[349] J. Girrbach, S. Mertens, U. Nierste, and S. Wiesenfeldt, Lepton flavour violation in the MSSM, JHEP 05 (2010) 026, arXiv:0910.2663.

[350] A. Falkowski, D. M. Straub, and A. Vicente, Vector-like leptons: Higgs decays and collider phenomenology, arXiv:1312.5329.

[351] A. Crivellin, S. Najjari, and J. Rosiek, Lepton Flavor Violation in the Standard Model with general Dimension-Six Operators, arXiv:1312.0634.

[352] W. Altmannshofer, R. Harnik, and J. Zupan, Low Energy Probes of PeV Scale Sfermions, JHEP 1311 (2013) 202, arXiv:1308.3653.

[353] M. Blanke, A. J. Buras, B. Duling, A. Poschenrieder, and C. Tarantino, Charged Lepton Flavour Violation and $(g-2)_{\mu}$ in the Littlest Higgs Model with T-Parity: a clear Distinction from Supersymmetry, JHEP 05 (2007) 013, hep-ph/0702136.

[354] J. R. Ellis, J. Hisano, M. Raidal, and Y. Shimizu, A new parametrization of the seesaw mechanism and applications in supersymmetric models, Phys. Rev. D66 (2002) 115013, [hep-ph/0206110|.

[355] E. Arganda and M. J. Herrero, Testing supersymmetry with lepton flavor violating tau and mu decays, Phys. Rev. D73 (2006) 055003, hep-ph/0510405].

[356] A. Brignole and A. Rossi, Anatomy and phenomenology of $\mu \tau$ lepton flavour violation in the MSSM, Nucl. Phys. B701 (2004) 3-53, hep-ph/0404211].

[357] P. Paradisi, Higgs-mediated $\tau \rightarrow \mu$ and $\tau \rightarrow e$ transitions in II Higgs doublet model and supersymmetry, JHEP 02 (2006) 050, hep-ph/0508054. 
[358] P. Paradisi, Higgs-mediated $e \rightarrow \mu$ transitions in II Higgs doublet model and supersymmetry, JHEP 08 (2006) 047, hep-ph/0601100.

[359] P. Paradisi, Constraints on SUSY lepton flavour violation by rare processes, JHEP 10 (2005) 006, hep-ph/0505046.

[360] F. del Aguila, J. I. Illana, and M. D. Jenkins, Precise limits from lepton flavour violating processes on the Littlest Higgs model with T-parity, JHEP 01 (2009) 080, arXiv:0811.2891.

[361] T. Goto, Y. Okada, and Y. Yamamoto, Tau and muon lepton flavor violations in the littlest Higgs model with T-parity, Phys.Rev. D83 (2011) 053011, arXiv:1012.4385.

[362] A. J. Buras, B. Duling, T. Feldmann, T. Heidsieck, and C. Promberger, Lepton Flavour Violation in the Presence of a Fourth Generation of Quarks and Leptons, JHEP 1009 (2010) 104, arXiv:1006.5356.

[363] MEG Collaboration Collaboration, J. Adam et. al., New constraint on the existence of the $\mu^{+} \rightarrow e^{+} \gamma$ decay, Phys.Rev.Lett. 110 (2013) 201801, arXiv:1303.0754.

[364] A. Baldini, F. Cei, C. Cerri, S. Dussoni, L. Galli, et. al., MEG Upgrade Proposal, arXiv:1301.7225.

[365] A. Blondel, A. Bravar, M. Pohl, S. Bachmann, N. Berger, et. al., Research Proposal for an Experiment to Search for the Decay $\mu \rightarrow$ eee, arXiv:1301.6113.

[366] R. Barlow, The PRISM/PRIME project, Nucl.Phys.Proc.Suppl. 218 (2011) 44-49.

[367] SINDRUM II Collaboration Collaboration, J. Kaulard et. al., Improved limit on the branching ratio of $\mu \rightarrow e$ conversion on titanium, Phys.Lett. B422 (1998) 334-338.

[368] Mu2e Collaboration Collaboration, R. Abrams et. al., Mu2e Conceptual Design Report, arXiv:1211.7019.

[369] V. Cirigliano, R. Kitano, Y. Okada, and P. Tuzon, On the model discriminating power of $\mu \rightarrow$ e conversion in nuclei, Phys.Rev. D80 (2009) 013002, arXiv:0904.0957.

[370] T. Feldmann, Lepton Flavour Violation Theory, PoS BEAUTY2011 (2011) 017, $\operatorname{arXiv:1105.2139.}$.

[371] A. Ibarra, Neutrino physics and lepton flavour violation: A theoretical overview, Nuovo Cim. C033N5 (2010) 67-75. 
[372] T. Kinoshita and M. Nio, Improved $\alpha^{4}$ term of the muon anomalous magnetic moment, Phys.Rev. D70 (2004) 113001, hep-ph/0402206].

[373] M. Passera, Precise mass-dependent QED contributions to leptonic g-2 at order $\alpha^{2}$ and $\alpha^{3}$, Phys.Rev. D75 (2007) 013002, [hep-ph/0606174].

[374] T. Aoyama, M. Hayakawa, T. Kinoshita, and M. Nio, Tenth-Order QED Contribution to the Electron g-2 and an Improved Value of the Fine Structure Constant, Phys.Rev.Lett. 109 (2012) 111807, arXiv:1205.5368.

[375] T. Aoyama, M. Hayakawa, T. Kinoshita, and M. Nio, Complete Tenth-Order QED Contribution to the Muon g-2, Phys.Rev.Lett. 109 (2012) 111808, arXiv:1205.5370.

[376] A. Czarnecki, W. J. Marciano, and A. Vainshtein, Refinements in electroweak contributions to the muon anomalous magnetic moment, Phys.Rev. D67 (2003) 073006, hep-ph/0212229.

[377] J. Prades, E. de Rafael, and A. Vainshtein, Hadronic Light-by-Light Scattering Contribution to the Muon Anomalous Magnetic Moment, arXiv:0901.0306.

[378] J. Prades, Standard Model Prediction of the Muon Anomalous Magnetic Moment, Acta Phys.Polon.Supp. 3 (2010) 75-86, arXiv:0909.2546.

[379] M. Benayoun, P. David, L. DelBuono, and F. Jegerlehner, An Update of the HLS Estimate of the Muon g-2, Eur.Phys.J. C73 (2013) 2453, arXiv:1210.7184].

[380] Muon G-2 Collaboration, G. W. Bennett et. al., Final report of the muon E821 anomalous magnetic moment measurement at BNL, Phys. Rev. D73 (2006) 072003, hep-ex/0602035.

[381] D. Stockinger, $(g-2)_{\mu}$ and supersymmetry: Status and prospects, arXiv:0710.2429.

[382] S. Marchetti, S. Mertens, U. Nierste, and D. Stockinger, $\tan \beta$-enhanced supersymmetric corrections to the anomalous magnetic moment of the muon, Phys.Rev. D79 (2009) 013010, arXiv:0808.1530|.

[383] F. Feroz, B. C. Allanach, M. Hobson, S. S. AbdusSalam, R. Trotta, et. al., Bayesian Selection of sign $(\mu)$ within $m S U G R A$ in Global Fits Including WMAP5 Results, JHEP 0810 (2008) 064, arXiv:0807.4512].

[384] M. Nojiri, T. Plehn, G. Polesello, J. M. Alexander, B. Allanach, et. al., Physics Beyond the Standard Model: Supersymmetry, arXiv:0802.3672.

[385] G. Degrassi and G. Giudice, QED logarithms in the electroweak corrections to the muon anomalous magnetic moment, Phys.Rev. D58 (1998) 053007, hep-ph/9803384. 
[386] S. Heinemeyer, D. Stockinger, and G. Weiglein, Two loop SUSY corrections to the anomalous magnetic moment of the muon, Nucl.Phys. B690 (2004) 62-80, hep-ph/0312264.

[387] S. Heinemeyer, D. Stockinger, and G. Weiglein, Electroweak and supersymmetric two-loop corrections to $(g-2)_{\mu}$, Nucl.Phys. B699 (2004) 103-123, hep-ph/0405255].

[388] A. Crivellin, J. Girrbach, and U. Nierste, Yukawa coupling and anomalous magnetic moment of the muon: an update for the LHC era, Phys.Rev. D83 (2011) 055009, arXiv:1010.4485.

[389] F. Jegerlehner, Implications of low and high energy measurements on SUSY models, Frascati Phys.Ser. 54 (2012) 42-51, arXiv:1203.0806].

[390] D. Hanneke, S. Fogwell, and G. Gabrielse, New Measurement of the Electron Magnetic Moment and the Fine Structure Constant, Phys.Rev.Lett. 100 (2008) 120801, arXiv:0801.1134.

[391] T. Aoyama, M. Hayakawa, T. Kinoshita, and M. Nio, Revised value of the eighth-order QED contribution to the anomalous magnetic moment of the electron, Phys.Rev. D77 (2008) 053012, arXiv:0712.2607].

[392] P. Clade, E. de Mirandes, M. Cadoret, S. Guellati-Khelifa, C. Schwob, et. al., Determination of the Fine Structure Constant Based on Bloch Oscillations of Ultracold Atoms in a Vertical Optical Lattice, Phys.Rev.Lett. 96 (2006) 033001.

[393] M. Pospelov and A. Ritz, Electric dipole moments as probes of new physics, Annals Phys. 318 (2005) 119-169, hep-ph/0504231.

[394] B. Batell, Flavor-diagonal CP violation, Eur.Phys.J. C72 (2012) 2127.

[395] J. Hudson, D. Kara, I. Smallman, B. Sauer, M. Tarbutt, et. al., Improved measurement of the shape of the electron, Nature 473 (2011) 493-496.

[396] ACME Collaboration Collaboration, J. Baron et. al., Order of Magnitude Smaller Limit on the Electric Dipole Moment of the Electron, Science (2013) arXiv:1310.7534.

[397] X.-G. He, C.-J. Lee, S.-F. Li, and J. Tandean, A Large Electron EDM and Minimal Flavor Violation, arXiv:1401.2615.

[398] D. E. Morrissey and M. J. Ramsey-Musolf, Electroweak baryogenesis, New J.Phys. 14 (2012) 125003, arXiv:1206.2942.

[399] J. Kozaczuk, S. Profumo, M. J. Ramsey-Musolf, and C. L. Wainwright, Supersymmetric Electroweak Baryogenesis Via Resonant Sfermion Sources, Phys.Rev. D86 (2012) 096001, arXiv:1206.4100]. 
[400] Y. Li, S. Profumo, and M. Ramsey-Musolf, Bino-driven Electroweak Baryogenesis with highly suppressed Electric Dipole Moments, Phys.Lett. B673 (2009) 95-100, arXiv:0811.1987.

[401] T. Liu, M. J. Ramsey-Musolf, and J. Shu, Electroweak Beautygenesis: From $b \rightarrow s$ CP-violation to the Cosmic Baryon Asymmetry, Phys.Rev.Lett. 108 (2012) 221301, arXiv:1109.4145.

[402] S. Tulin and P. Winslow, Anomalous B meson mixing and baryogenesis, Phys.Rev. D84 (2011) 034013, arXiv:1105.2848.

[403] J. M. Cline, K. Kainulainen, and M. Trott, Electroweak Baryogenesis in Two Higgs Doublet Models and B meson anomalies, JHEP 1111 (2011) 089, arXiv:1107.3559.

[404] M. Jung and A. Pich, Electric Dipole Moments in Two-Higgs-Doublet Models, arXiv:1308.6283.

[405] F. Pisano and V. Pleitez, An SU(3) x U(1) model for electroweak interactions, Phys.Rev. D46 (1992) 410-417, [hep-ph/9206242].

[406] P. H. Frampton, Chiral dilepton model and the flavor question, Phys. Rev. Lett. 69 (1992) 2889-2891.

[407] J. T. Liu and D. Ng, Lepton flavor changing processes and CP violation in the 331 model, Phys.Rev. D50 (1994) 548-557, hep-ph/9401228.

[408] R. A. Diaz, R. Martinez, and F. Ochoa, $S U(3)(c) x S U(3)(L) x U(1)(X)$ models for beta arbitrary and families with mirror fermions, Phys.Rev. D72 (2005) 035018, hep-ph/0411263.

[409] J. T. Liu, Generation nonuniversality and flavor changing neutral currents in the 331 model, Phys.Rev. D50 (1994) 542-547, hep-ph/9312312].

[410] J. A. Rodriguez and M. Sher, FCNC and rare $B$ decays in 3-3-1 models, Phys.Rev. D70 (2004) 117702, hep-ph/0407248.

[411] C. Promberger, S. Schatt, and F. Schwab, Flavor Changing Neutral Current Effects and CP Violation in the Minimal 3-3-1 Model, Phys.Rev. D75 (2007) 115007, hep-ph/0702169.

[412] J. Agrawal, P. H. Frampton, and J. T. Liu, The Decay $b \rightarrow s \gamma$ in the 3-3-1 model, Int.J.Mod.Phys. A11 (1996) 2263-2280, hep-ph/9502353.

[413] C. Promberger, S. Schatt, F. Schwab, and S. Uhlig, Bounding the Minimal 331 Model through the Decay $B \rightarrow X_{s} \gamma$, Phys.Rev. D77 (2008) 115022, arXiv:0802.0949. 
[414] A. Machado, J. Montero, and V. Pleitez, FCNC in the minimal 3-3-1 model revisited, Phys.Rev. D88 (2013) 113002, arXiv:1305.1921.

[415] A. J. Buras, F. De Fazio, and J. Girrbach-Noe, Z-Z' Mixing and Z-Mediated $F C N C s$ in $S U(3)_{C} \times S U(3)_{L} \times U(1)_{X}$ Models, arXiv:1405.3850.

[416] O. Eberhardt, G. Herbert, H. Lacker, A. Lenz, A. Menzel, et. al., Impact of a Higgs boson at a mass of $126 \mathrm{GeV}$ on the standard model with three and four fermion generations, Phys.Rev.Lett. 109 (2012) 241802, arXiv:1209.1101.

[417] O. Eberhardt, U. Nierste, and M. Wiebusch, Status of the two-Higgs-doublet model of type II, arXiv:1305.1649.

[418] A. Celis, V. Ilisie, and A. Pich, LHC constraints on two-Higgs doublet models, JHEP 1307 (2013) 053, arXiv:1302.4022].

[419] C.-W. Chiang and K. Yagyu, Implications of Higgs boson search data on the two-Higgs doublet models with a softly broken $Z_{2}$ symmetry, JHEP 1307 (2013)

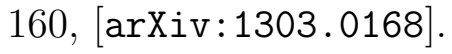

[420] A. Barroso, P. Ferreira, I. Ivanov, and R. Santos, Metastability bounds on the two Higgs doublet model, JHEP 1306 (2013) 045, arXiv:1303.5098.

[421] B. Grinstein and P. Uttayarat, Carving Out Parameter Space in Type-II Two Higgs Doublets Model, JHEP 1306 (2013) 094, arXiv:1304.0028].

[422] T. Moroi, CP violation in $B_{d} \rightarrow \phi K_{S}$ in SUSY GUT with right-handed neutrinos, Phys.Lett. B493 (2000) 366-374, hep-ph/0007328.

[423] D. Chang, A. Masiero, and H. Murayama, Neutrino mixing and large CP violation in B physics, Phys.Rev. D67 (2003) 075013, hep-ph/0205111.

[424] J. Girrbach, S. Jager, M. Knopf, W. Martens, U. Nierste, et. al., Flavor Physics in an SO(10) Grand Unified Model, JHEP 1106 (2011) 044, arXiv:1101.6047.

[425] J. Girrbach, Flavour Physics in an SO(10) Grand Unified Model, PoS EPS-HEP2011 (2011) 183, arXiv:1108.4852.

[426] U. Nierste, Flavour physics, supersymmetry and grand unification, arXiv:1107.0621.

[427] U. Nierste, "Impact of the higgs discovery on two models of new physics." Talk given at Portoroz, 14.-18. April 2013.

[428] U. Nierste and J. Stockel, "work in progress." 2013.

[429] A. Crivellin, Effects of right-handed charged currents on the determinations of $\left|V_{u b}\right|$ and $\left|V_{c b}\right|$, Phys.Rev. D81 (2010) 031301, |arXiv:0907.2461. 
[430] C.-H. Chen and S.-h. Nam, Left-right mixing on leptonic and semileptonic $b \rightarrow u$ decays, Phys.Lett. B666 (2008) 462-466, arXiv:0807.0896].

[431] A. Crivellin and L. Mercolli, $B \rightarrow X_{d} \gamma$ and constraints on new physics, Phys.Rev. D84 (2011) 114005, arXiv:1106.5499.

[432] K. Agashe, A. Delgado, M. J. May, and R. Sundrum, Rs1, custodial isospin and precision tests, JHEP 08 (2003) 050, hep-ph/0308036.

[433] C. Csaki, C. Grojean, L. Pilo, and J. Terning, Towards a realistic model of higgsless electroweak symmetry breaking, Phys. Rev. Lett. 92 (2004) 101802, hep-ph/0308038.

[434] K. Agashe, R. Contino, L. Da Rold, and A. Pomarol, A custodial symmetry for Zbb̆, Phys. Lett. B641 (2006) 62-66, [hep-ph/0605341].

[435] M. Blanke, A. J. Buras, B. Duling, S. Gori, and A. Weiler, $\Delta F=2$ Observables and Fine-Tuning in a Warped Extra Dimension with Custodial Protection, JHEP 03 (2009) 001, arXiv:0809.1073.

[436] S. Casagrande, F. Goertz, U. Haisch, M. Neubert, and T. Pfoh, Flavor Physics in the Randall-Sundrum Model: I. Theoretical Setup and Electroweak Precision Tests, JHEP 10 (2008) 094, arXiv:0807.4937.

[437] M. Bauer, S. Casagrande, L. Grunder, U. Haisch, and M. Neubert, Little Randall-Sundrum models: $\varepsilon_{K}$ strikes again, Phys.Rev. D79 (2009) 076001, arXiv:0811.3678.

[438] M. Bauer, S. Casagrande, U. Haisch, and M. Neubert, Flavor Physics in the Randall-Sundrum Model: II. Tree-Level Weak-Interaction Processes, JHEP 1009 (2010) 017, arXiv:0912.1625.

[439] O. Gedalia, G. Isidori, and G. Perez, Combining Direct and Indirect Kaon CP Violation to Constrain the Warped KK Scale, Phys.Lett. B682 (2009) 200-206, arXiv:0905.3264.

[440] K. Agashe, G. Perez, and A. Soni, Flavor structure of warped extra dimension models, Phys. Rev. D71 (2005) 016002, hep-ph/0408134.

[441] E. O. Iltan, The effects of lepton KK modes on the lepton electric dipole moments in the Randall Sundrum scenario, Eur. Phys. J. C54 (2008) 583-590, arXiv:0708.3765].

[442] K. Agashe, A. E. Blechman, and F. Petriello, Probing the Randall-Sundrum geometric origin of flavor with lepton flavor violation, Phys. Rev. D74 (2006) 053011, hep-ph/0606021]. 
[443] S. Davidson, G. Isidori, and S. Uhlig, Solving the flavour problem with hierarchical fermion wave functions, Phys. Lett. B663 (2008) 73-79, arXiv:0711.3376.

[444] K. Agashe, Relaxing Constraints from Lepton Flavor Violation in 5D Flavorful Theories, Phys.Rev. D80 (2009) 115020, arXiv:0902.2400.

[445] C. Csaki, Y. Grossman, P. Tanedo, and Y. Tsai, Warped penguin diagrams, Phys.Rev. D83 (2011) 073002, arXiv:1004.2037.

[446] M. Blanke, B. Shakya, P. Tanedo, and Y. Tsai, The Birds and the Bs in RS: The $b \rightarrow$ s $\gamma$ penguin in a warped extra dimension, JHEP 1208 (2012) 038, arXiv:1203.6650.

[447] K. Agashe, A. Azatov, and L. Zhu, Flavor Violation Tests of Warped/Composite SM in the Two-Site Approach, Phys. Rev. D79 (2009) 056006, arXiv:0810.1016].

[448] D. B. Kaplan, Flavor at SSC energies: A New mechanism for dynamically generated fermion masses, Nucl.Phys. B365 (1991) 259-278.

[449] Y. Grossman and M. Neubert, Neutrino masses and mixings in non-factorizable geometry, Phys. Lett. B474 (2000) 361-371, hep-ph/9912408.

[450] S. J. Huber and Q. Shafi, Fermion masses, mixings and proton decay in a randall-sundrum model, Phys. Lett. B498 (2001) 256-262, hep-ph/0010195.

[451] T. Gherghetta and A. Pomarol, Bulk fields and supersymmetry in a slice of ads, Nucl. Phys. B586 (2000) 141-162, hep-ph/0003129.

[452] C. Csaki, A. Falkowski, and A. Weiler, The Flavor of the Composite Pseudo-Goldstone Higgs, JHEP 09 (2008) 008, arXiv:0804.1954.

[453] G. Cacciapaglia et. al., A GIM Mechanism from Extra Dimensions, JHEP 04 (2008) 006, arXiv:0709.1714.

[454] R. Barbieri, G. Isidori, and D. Pappadopulo, Composite fermions in Electroweak Symmetry Breaking, JHEP 0902 (2009) 029, arXiv:0811.2888.

[455] M. Redi and A. Weiler, Flavor and CP Invariant Composite Higgs Models, JHEP 1111 (2011) 108, arXiv:1106.6357].

[456] M. Redi, Composite MFV and Beyond, Eur.Phys.J. C72 (2012) 2030, arXiv:1203.4220.

[457] A. L. Fitzpatrick, G. Perez, and L. Randall, Flavor anarchy in a Randall-Sundrum model with 5D minimal flavor violation and a low Kaluza-Klein scale, Phys.Rev.Lett. 100 (2008) 171604, arXiv:0710.1869]. 
[458] J. Santiago, Minimal Flavor Protection: A New Flavor Paradigm in Warped Models, JHEP 12 (2008) 046, arXiv:0806.1230].

[459] C. Csaki, A. Falkowski, and A. Weiler, A Simple Flavor Protection for RS, Phys.Rev. D80 (2009) 016001, arXiv:0806.3757].

[460] M. Bauer, R. Malm, and M. Neubert, A Solution to the Flavor Problem of Warped Extra-Dimension Models, Phys.Rev.Lett. 108 (2012) 081603, arXiv:1110.0471.

[461] D. M. Straub, Anatomy of flavour-changing Z couplings in models with partial compositeness, JHEP 1308 (2013) 108, arXiv:1302.4651.

[462] R. Contino, T. Kramer, M. Son, and R. Sundrum, Warped/composite phenomenology simplified, JHEP 05 (2007) 074, hep-ph/0612180|.

[463] R. Barbieri, D. Buttazzo, F. Sala, D. M. Straub, and A. Tesi, A 125 GeV composite Higgs boson versus flavour and electroweak precision tests, JHEP 1305 (2013) 069, arXiv:1211.5085.

[464] B. Grinstein, M. Redi, and G. Villadoro, Low Scale Flavor Gauge Symmetries, JHEP 1011 (2010) 067, arXiv:1009.2049].

[465] T. Feldmann, See-Saw Masses for Quarks and Leptons in SU(5), JHEP 1104 (2011) 043, arXiv:1010.2116.

[466] A. J. Buras, C. Grojean, S. Pokorski, and R. Ziegler, FCNC Effects in a Minimal Theory of Fermion Masses, JHEP 1108 (2011) 028, arXiv:1105.3725. 